\title{
Investigation of the Double-Y Balun for Feeding Pulsed Antennas
}

\author{
A Thesis \\ Presented to \\ The Academic Faculty \\ by \\ Jaikrishna Benny Venkatesan
}

\author{
In Partial Fulfillment \\ of the Requirements for the Degree \\ Doctor of Philosophy
}

School of Electrical and Computer Engineering

Georgia Institute of Technology

July 2004 


\section{Investigation of the Double-Y Balun for Feeding Pulsed Antennas}

Approved by:

Dr. Waymond Scott, Committee Chair Dr. Robert K. Feeney

Dr. Glenn Smith

Dr. Guillermo Goldsztein

Dr. Marshall Leach, Jr.

Date Approved: 6 July 2003 
To my parents, Dr. K. Venkatesan and Anuradha Venkatesan and my sister, Charu Venkatesan, MD. 


\section{ACKNOWLEDGEMENTS}

I would like to express my deepest gratitude to Dr. Waymond Scott, for serving as my guide and mentor. Over the course of my graduate studies, I have acquired an immense wealth of knowledge from Dr. Scott, particularly on measurement techniques and conducting practical experimental work.

I would like to thank Dr. Marshall Leach, who has been a source of encouragement throughout my undergraduate and graduate years, here at Georgia Tech. I have learned so much from his many classes. I would like thank to Dr. Robert K. Feeney and Dr. David Hertling, whose undergraduate RF classes sparked my interest in the area of $\mathrm{RF} /$ Electromagnetics and allowed me to excel at my first job, as an RF Engineer, at Motorola. Many thanks to Dr. Glenn Smith for his discussions on this topic. I would also like to thank Dr. Thomas Gaylord for his assistance with the optical link constructed in this research. I would like to thank Dr. Goldsztein for serving on my thesis committee.

I would like to thank the former and current members of the electromagnetics research group: Dr. Christoph Schroeder, Dr. Thorsten Hertel, Dr. Rickard Petersson, Dr. Kangwook Kim, Mr. Ricardo Lopez, Mr. Pelham Norville, and Mr. David Reid, for all the interesting exchange of ideas we have shared over the years. Thanks to Mr. Richard Todd Lee for sharing his Matlab code to generate 3-D patterns. I would like to thank Taconic, Dielectric Laboratories, and Maxim for donating parts used in conducting this research.

Finally, I would like to thank my parents, my sister, Paul Singh, and Michael Delafchell, for their constant support, guidance, and encouragement over the years.

This work was supported in part by the US Army Night Vision Electronic Sensors 
Directorate, Science and Technology Division, Countermine Branch. 


\section{TABLE OF CONTENTS}

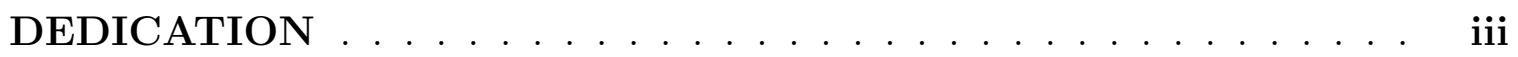

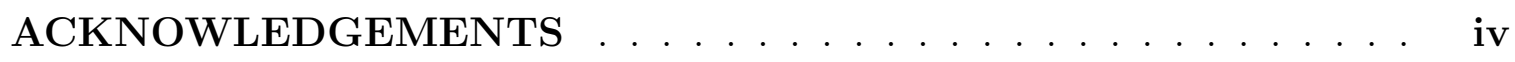

LIST OF TABLES $\ldots \ldots \ldots \ldots \ldots \ldots$ viii

LIST OF FIGURES $\ldots \ldots \ldots \ldots \ldots \ldots \ldots \ldots$ ix

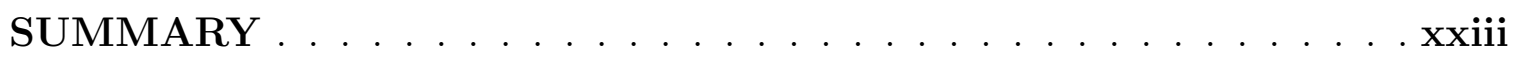

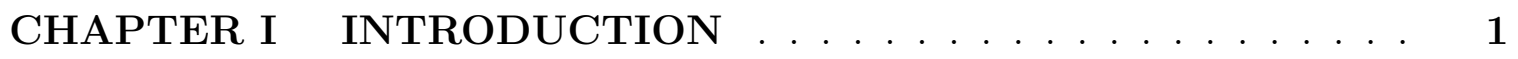

CHAPTER II DOUBLE-Y BALUN - NUMERICAL ANALYSIS . 17

$2.1 \mathrm{CPW} / \mathrm{CPS}$ Lines . . . . . . . . . . . . . . . . . . . 17

2.2 Momentum vs. High Frequency Structure Simulator (HFSS) . . . 26

2.3 Resonant Modes . . . . . . . . . . . . . . . . . . . . 37

$2.3 .1 \quad \lambda / 8$ Resonance . . . . . . . . . . . . . . . 37

2.3 .2 CPW Resonance . . . . . . . . . . . . . . . . . 43

2.3 .3 Dipole Resonance . . . . . . . . . . . . . . . . . . 46

2.4 CPW Bridge Design-Substrate Thickness _. . . . . . . . . . 47

2.5 Aspect Ratio . . . . . . . . . . . . . . . . . 56

2.6 Stub Lengths . . . . . . . . . . . . . . . . . . . . . . 59

2.6 .1 Compensation . . . . . . . . . . . . . . . . 59

2.7 Double-Y Balun Design . . . . . . . . . . . . . . . . . 60

\section{CHAPTER III MODELING BALANCED/UNBALANCED DIPOLE}

IN NEC . . . . . . . . . . . . . . . . . . . . . 66

3.1 Balanced/Unbalanced Dipole Currents . . . . . . . . . . . . 68

3.2 Dipole Amplitude Patterns . . . . . . . . . . . . . . . . . 73

3.3 Adding Wu-King Loading . . . . . . . . . . . . . . . . . . . 89

3.4 Fields of Dipole and Travelling-Wave Antenna . . . . . . . . . 95

3.5 Time-Domain Analysis . . . . . . . . . . . . . . . . . . 98

3.5.1 Time Gating Reflected Pulses _ . . . . . . . . . . 98 
3.5.2 Measuring Cross-Polarized Component . . . . . . . . . . . . 112

CHAPTER IV OPTICAL LINK . . . . . . . . . . . . . . . 120

4.1 Motivation ..................... 120

4.2 Laser Modulator Unit $(\mathrm{LM})$. . . . . . . . . . . . . . . . . . 122

4.3 Laser Receiver Unit $(\mathrm{LR})$. . . . . . . . . . . . . . . . . . . 123

CHAPTER V EXPERIMENTAL WORK . . . . . . . . . . . 132

5.1 Measured S-Parameters of Double-Y Balun . . . . . . . . . . . . . . 132

5.2 Antenna Pattern Measurements . . . . . . . . . . . . . 141

5.2.1 5 cm Dipole Azimuth Patterns Without Optical Link . . . . 142

5.2.2 12 cm Dipole Patterns Without Optical Link . . . . . . . . . 154

$5.2 .35 \mathrm{~cm}$ Dipole Patterns with Optical Link . . . . . . . . . . . . 162

5.2.4 12 cm Dipole Patterns with Optical Link . . . . . . . . . . . 174

5.2.5 Resistively Loaded V-Dipole . . . . . . . . . . . . . . . 181

CHAPTER VI CONCLUSION . . . . . . . . . . . . . . . 196

APPENDIX A - GENERALIZED 2-PORT S-PARAMETERS . 203

REFERENCES . . . . . . . . . . . . . . . . . . 205

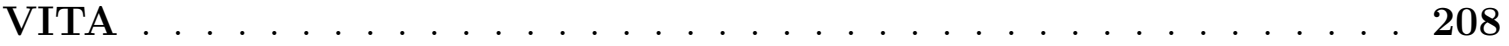




\section{LIST OF TABLES}

Table 1 Momentum simulation summary for balun modeled as illustrated in Fig. 31. . . . . . . . . . . . . . . . . . . . .

Table 2 Computed parameters for Wu-King loading along feedline of radius $0.0001 \mathrm{~m}$. . . . . . . . . . . . . . . . . . . . . . 89

Table 3 Optical characteristics of the Alcatel 1916LMM laser module [1]. . 124

Table 4 Optical and electrical characteristics of the Alcatel 1915 DMO receiver module [2]. . . . . . . . . . . . . . . . . . . . . . . . . . 128 


\section{LIST OF FIGURES}

Figure 1 Illustration of differential currents along (a) a two-wire transmission line and (b) a dipole fed by a two-wire transmission line. . . . . . . 2

Figure 2 Illustration of a coaxial line. . . . . . . . . . . . . . . . 2

Figure 3 Cross section of (a) asymmetric coaxial line and (b) symmetric twowire transmission line. The coaxial line is defined as an unbalanced structure, and the two-wire transmission line is defined as a balanced structure. . . . . . . . . . . . . . . . 3

Figure 4 Illustration of dipole fed directly by a coaxial line. . . . . . . . . . . 4

Figure 5 Balun constructed by tapering outer conductor of coaxial cable. . . 5

Figure 6 Illustration of the Guanella balun $[3] \ldots \ldots$. . . . . . . . . . . . 6

Figure 7 Equivalent lumped element model of the Guanella balun. . . . . . . 6

Figure 8 Illustration of coaxial line loaded with ferrite beads and feeding dipole. 7

Figure 9 Magnetic flux induced in ferrite bead due to (a) common-mode and (b) differential-mode currents. . . . . . . . . . . . . . . 7

Figure 10 Balun employing phase shift to achieve balancing. . . . . . . . . . . 8

Figure 11 Illustration of the sleeve balun placed around a coaxial line feeding a dipole. . . . . . . . . . . . . . . . . . . . 9 9

Figure 12 Illustration of (a) the lattice balun implemented with lumped element components and (b) operation of circuit as a balun. . . . . . . 10

Figure 13 Illustration of (a) the double-y balun with input/output CPW and CPS lines, (b) the double-y junction where the mode conversion occurs between CPW and CPS lines, and (c) reference points used to measure the length of the CPW and CPS stubs. . . . . . . .

Figure 14 Illustration of (a) the double-y junction with an input signal applied to Port 1, (b) the double-y junction with an input signal applied to Port 2, and (c) the double-y balun. In (a) and (b), $\Gamma$ is the reflection coefficient at each port, and $\phi$ is the phase of the incident signal at the port. . . . . . . . . . . . . . . .

Figure 15 Illustration of (a) the equivalent circuit model of the double-y balun and (b) equivalent circuit model of double-y balun with stub lengths equal to odd multiples of $\lambda / 8 \ldots \ldots \ldots 15$

Figure 16 Illustration of (a) CPW and (b) CPS lines. . . . . . . . . . . . 18 
Figure 17 Cross-section view of (a) CPW and (b) CPS lines. . . . . . . . 18

Figure 18 Double-y balun implemented with CPW and CPS lines. . . . . . 18

Figure 19 Effect of substrate thickness on CPW characteristic impedance. . . 19

Figure 20 Effect of ground conductor width on CPW characteristic impedance. 20

Figure 21 Effect of substrate permittivity on CPW characteristic impedance. 20

Figure 22 Effect of substrate thickness on CPS characteristic impedance. . . . 21

Figure 23 Effect of substrate permittivity on CPS characteristic impedance. . 22

Figure 24 Total attenuation $\left(\alpha_{d}+\alpha_{c}\right)$ computed using Equations 14 and 15 for CPW line in Fig. 17a. . . . . . . . . . . . . . . . .

Figure 25 Synthesized gap and outer conductor widths for CPW line in Fig. $17 \mathrm{a}$, with characteristic impedances and center conductor widths illustrated in Fig. 24. . . . . . . . . . . . . . . . . . . . . . .

Figure 26 Total attenuation $\left(\alpha_{d}+\alpha_{c}\right)$ computed using Equations 14 and 15 for CPS line in Fig. 17b. . . . . . . . . . . . . . . . . . .

Figure 27 Synthesized gap and conductor widths for CPS line in Fig. 17b, with characteristic impedances and gap widths illustrated in Fig. 26. . .

Figure 28 Design approach to transition from coaxial feedline to double-y balun and resistively loaded V-dipole. . . . . . . . . . . . 26

Figure 29 Dimensions of double-y balun modeled in Momentum and HFSS. . 27

Figure 30 Plot illustrating impedance vs. minimum achievable total width of CPW and CPS lines over 58 mil FR4 substrate subject to following constraints: minimum $\mathrm{w}_{c c p w}=10$ mils, minimum $\mathrm{g}_{c p s}=6$ mils. . . . 28

Figure 31 Double-y balun modeled and meshed in Momentum. . . . . . . . . 29

Figure 32 Illustration of (a) 3-D view of double-y balun and (b) substrate characteristics as defined in Momentum. . . . . . . . . . . . .

Figure 33 Momentum $\mathrm{s}_{11}$ results for two different meshes (mesh generated at

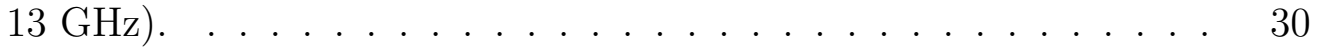

Figure 34 Momentum $\mathrm{s}_{21}$ results for two different meshes (mesh generated at

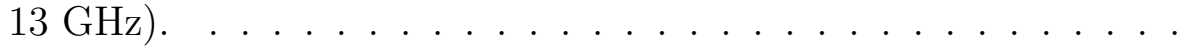

Figure 35 Illustration of (a) top and (b)3-D view of double-y balun modeled in HFSS. . . . . . . . . . . . . . . . . .

Figure 36 Illustration of (a) cross-section and (b) top view of double-y balun modeled in HFSS within bounded region. 
Figure 37 HFSS $s_{11}$ results for double-y balun in Fig. 29 with various PML

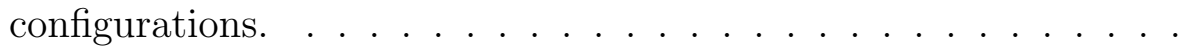

Figure 38 HFSS s$_{21}$ results for double-y balun in Fig. 29 with various PML

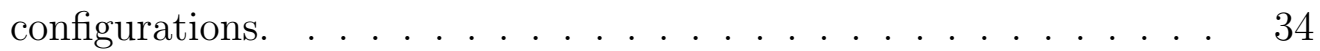

Figure 39 Momentum vs. HFSS $s_{11}$ results for double-y balun in Fig. 29. . . . 35

Figure 40 Momentum vs. HFSS $s_{21}$ results for double-y balun in Fig. 29. . . . 36

Figure 41 Illustration of (a) double-y balun implemented with CPW and CPS lines and (b) equivalent lumped element model. . . . . . . . . . .

Figure 42 Magnitude of input impedance looking into CPW section of balun junction. Balun is matched at all frequencies when characteristic impedances of CPW and CPS stubs are equal to the terminated load (normalized to $1 \Omega)$. . . . . . . . . . . . .

Figure 43 ADS implementation of the double-y balun lumped element model illustrated in Fig. 41. . . . . . . . . . . . . . . . . .

Figure 44 Plot of $\mathrm{s}_{21}$ comparing theoretical results from Equation 18 with ADS model. Theoretical and ADS curves overlap for the matched case. Theoretical and ADS curves for the mismatched case also overlap. .

Figure 45 Momentum $\mathrm{s}_{21}$ results for double-y balun with 80 mil and 110 mil stubs. . . . . . . . . . . . . . . .

Figure 46 Illustration of (a) differential-mode currents along outer CPW ground conductors and (b) equivalent transmission line circuit. . . . . . .

Figure 47 Illustration of double-y balun with additional CPW bridge to remove $\mathrm{CPW}$ resonance along outer ground conductors. . . . . . . . . . .

Figure 48 Momentum $\mathrm{s}_{21}$ results for double-y balun with and without additional CPW bridge. . . . . . . . . . . . . . . . . 45

Figure 49 Illustration of double-y balun with extended CPW and CPS sections. 45

Figure 50 Momentum $\mathrm{s}_{21}$ results for double-y balun in Fig. 49 with and without additional CPW bridges. . . . . . . . . . . . . . . . . . .

Figure 51 Illustration of 'dipole' resonance excited at balun junction, causing CPW and CPS lines to act as dipole arms. . . . . . . . . . . . .

Figure 52 Illustration of (a) 3-D drawing of CPW bridge modeled in Momentum (b) actual view of CPW bridge modeled in Momentum and (c) substrate characteristics as defined in Momentum. . . . . . . . . . .

Figure $53 \mathrm{Z}_{\text {inbridge }}$ computed using Momentum for 25 and 58 mil thick FR4 substrate. . . . . . . . . . . . . . . . . 51 
Figure 54 Momentum simulation results illustrating effect of via diameter on CPW bridge impedance. . . . . . . . . . . . . . . . . 51

Figure 55 Modified lumped element model for double-y balun. . . . . . . . . 52

Figure 56 Modified ADS lumped element model for double-y balun. . . . . . . 52

Figure 57 Illustration of shift in $\lambda / 8$ resonance from Momentum and ADS simulations for 58 mil substrate and 110 mil stubs. . . . . . . . . 53

Figure 58 Illustration of reflection at $\lambda / 8$ resonance from Momentum and ADS simulations for 58 mil substrate and 110 mil stubs. . . . . . . . .

Figure 59 Illustration of shift in $\lambda / 8$ resonance from Momentum and ADS simulations for 58 mil substrate and 80 mil stubs. . . . . . . . . . 54

Figure 60 Illustration of reflection at $\lambda / 8$ resonance from Momentum and ADS simulations for 58 mil substrate and 80 mil stubs. . . . . . . . 54

Figure 61 Illustration of shift in $\lambda / 8$ resonance from Momentum and ADS simulations for 25 mil substrate and 110 mil stubs. . . . . . . . . 55

Figure 62 Illustration of shift in $\lambda / 8$ resonance from Momentum and ADS simulations for 25 mil substrate and 80 mil stubs. . . . . . . . . 55

Figure 63 Momentum $\mathrm{s}_{21}$ plot for double-y balun over 25 and 58 mil FR4 substrate with 110 mil CPW and CPS stubs. . . . . . . . . . 56

Figure 64 Plot of $\mathrm{w}_{\text {cpwout }}$ for CPS and CPW minimizations. . . . . . . . 58

Figure 65 Double-y balun with (a) $\mathrm{w}_{\text {cpwin }}$ and (b) $\mathrm{w}_{\text {cpwout }}$ minimized. . . . . 59

Figure 66 Insertion loss obtained via Momentum simulation of double-y baluns in Fig. 65. . . . . . . . . . . . . . . . . . . . 59

Figure 67 Double-y balun designed with compensated stub lengths. . . . . . . 60

Figure 68 Momentum $\mathrm{s}_{21}$ results for double-y balun with and without compensated stub lengths. . . . . . . . . . . . . . .

Figure 69 Double-y balun junction designed, over 25 mil FR4 substrate, for use in experimental work. . . . . . . . . . . . .

Figure 70 Impedance profile along exponentially tapered CPW section of the double-y balun. . . . . . . . . . . . . . . . .

Figure 71 Illustration of CPW tapered section with extended ground conductors at coaxial line to $\mathrm{CPW}$ transition. . . . . . . . . . . . . . .

Figure 72 Synthesized CPW gap and conductor widths along exponentially tapered section. . . . . . . . . . . . . . . . . . . 
Figure 73 Impedance profile along exponentially tapered CPS section of the double-y balun. . . . . . . . . . . . . . . . . .

Figure 74 Illustration of exponentially tapered CPS section. . . . . . . . . . . 65

Figure 75 Synthesized CPS gap and conductor widths along exponentially tapered section. . . . . . . . . . . . . . . .

Figure 76 NEC model used to illustrate effects of unbalanced feed on a dipole's radiation pattern. . . . . . . . . . . . . . .

Figure 77 Normalized currents computed using NEC along balanced dipole arms at frequencies where the dipole length is $\lambda / 2,3 \lambda / 2$, and $5 \lambda / 2$.

Figure 78 Normalized currents computed using NEC along unbalanced dipole arms of length $\lambda / 2$ and with a feedline length of $5 \lambda / 4$. Currents are normalized to the maximum value obtained with $\alpha=0.5$. . . . . .

Figure 79 Normalized currents computed using NEC along unbalanced dipole arms of length $3 \lambda / 2$ and with a feedline length of $5 \lambda / 4$. Currents are normalized to the maximum value obtained with $\alpha=0.5$. . . .

Figure 80 Normalized currents computed using NEC along unbalanced dipole arms of length $5 \lambda / 2$ and with a feedline length of $5 \lambda / 4$. Currents are normalized to the maximum value obtained with $\alpha=0.5$. . . .

Figure 81 Normalized relative currents computed using NEC along feedline of unbalanced dipole for values of $\alpha$. Currents are normalized to the maximum value obtained with $\alpha=0.5$. . . . . . . . . . . .

Figure 82 Normalized relative currents computed using NEC along feedline, with height $h$, of unbalanced dipole. Currents are normalized to the maximum value obtained with $\mathrm{h}=5 \lambda / 4 \ldots \ldots$. . . . . . . . .

Figure 83 3-D normalized far field pattern, generated with NEC model in Fig. 76 , of (a) balanced dipole with $\alpha=0.5$ and (b) unbalanced dipole with $\alpha=0.1 \ldots$. . . . . . . . . . . . . . . . . . .

Figure 84 Illustration of (a) azimuth plane and (b) elevation plane with $\phi$ and $\theta$ defined in spherical coordinates. . . . . . . . . . . . .

Figure 85 Normalized amplitude patterns for balanced dipole at (a) $\lambda / 2$, (b) $3 \lambda / 2$, and (c) $5 \lambda / 2$ computed using the NEC model in Fig. 76 with

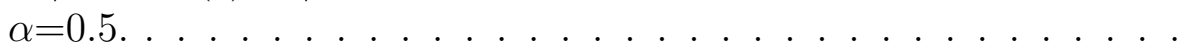

Figure 85 (Cont'd.) Normalized amplitude patterns for balanced dipole at (a) $\lambda / 2$, (b) $3 \lambda / 2$, and (c) $5 \lambda / 2$ computed using the NEC model in Fig.

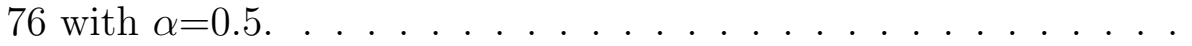


Figure 86 Normalized amplitude patterns for unbalanced dipole with $5 \lambda / 4$ feedline at (a) $\lambda / 2$, (b) $3 \lambda / 2$, and (c) $5 \lambda / 2$ computed using the NEC model in Fig. 76 with $\alpha=0.1$. . . . . . . . . . . . . . . . .

Figure 86 (Cont'd.) Normalized amplitude patterns for unbalanced dipole with $5 \lambda / 4$ feedline at (a) $\lambda / 2$, (b) $3 \lambda / 2$, and (c) $5 \lambda / 2$ computed using the NEC model in Fig. 76 with $\alpha=0.1$. . . . . . . . . . . . . . .

Figure 87 Normalized amplitude patterns for unbalanced dipole with $11 \lambda / 4$ feedline at (a) $\lambda / 2$, (b) $3 \lambda / 2$, and (c) $5 \lambda / 2$ computed using the NEC

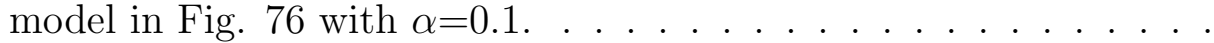

Figure 87 (Cont'd.) Normalized amplitude patterns for unbalanced dipole with $11 \lambda / 4$ feedline at (a) $\lambda / 2$, (b) $3 \lambda / 2$, and (c) $5 \lambda / 2$ computed using the NEC model in Fig. 76 with $\alpha=0.1$. . . . . . . . . . . . . .

Figure 88 Normalized amplitude pattern computed along azimuth plane, using the NEC model in Fig. 76 with 11 $\lambda / 4$ length feedline, for a balanced $(\alpha=0.5)$ dipole and an unbalanced $(\alpha=0.1)$ dipole. . . . . . . .

Figure 89 Normalized amplitude patterns along azimuth plane $\left(\left|E_{\phi}\right|\right)$ for a dipole with $11 \lambda / 4$ feedline at (a) $\lambda / 2$, (b) $3 \lambda / 2$, and (c) $5 \lambda / 2$, computed using the NEC model in Fig. 76 for several values of $\alpha$. . . .

Figure 89 (Cont'd.) Normalized amplitude patterns along azimuth plane $\left(\left|E_{\phi}\right|\right)$ for a dipole with $11 \lambda / 4$ feedline at (a) $\lambda / 2$, (b) $3 \lambda / 2$, and (c) $5 \lambda / 2$, computed using the NEC model in Fig. 76 for several values of $\alpha$. .

Figure 90 Normalized amplitude patterns along elevation plane $\left(\left|E_{\theta}\right|\right)$ component) for a dipole with $11 \lambda / 4$ feedline at (a) $\lambda / 2$, (b) $3 \lambda / 2$, and (c) $5 \lambda / 2$ computed using the NEC model in Fig. 76 for several values of $\alpha \ldots \ldots \ldots \ldots \ldots \ldots \ldots \ldots \ldots \ldots \ldots$

Figure 90 (Cont'd.) Normalized amplitude patterns along elevation plane $\left(\left|E_{\theta}\right|\right)$ component) for a dipole with $11 \lambda / 4$ feedline at (a) $\lambda / 2$, (b) $3 \lambda / 2$, and (c) $5 \lambda / 2$ computed using the NEC model in Fig. 76 for several values of $\alpha \ldots \ldots \ldots \ldots \ldots \ldots . \ldots \ldots$

Figure 91 NEC model in Fig. 76 modified to include Wu-King loading along feedline. The Wu-King loading profile is used to approximate an infinite feedline. . . . . . . . . . . . . . . . . . . 990

Figure 92 Wu-King loading profile along feedline of NEC model in Fig. $76 . \quad$ 91

Figure 93 3-D normalized far field pattern of unbalanced dipole generated using NEC model in Fig. 91 with $\alpha=0.1$ and Wu-King loading along feedline of length $21 \lambda / 4 \ldots \ldots$. . . . . . . . . . . . . . 
Figure 94 Normalized amplitude patterns for unbalanced dipole with Wu-King loading along feedline of length $21 \lambda / 4$, at (a) $\lambda / 2$, (b) $3 \lambda / 2$, and (c) $5 \lambda / 2$ computed using NEC model in Fig. $91 . \quad \ldots . . . . .$.

Figure 94 (Cont'd.) Normalized amplitude patterns for unbalanced dipole with Wu-King loading along feedline of length $21 \lambda / 4$ at (a) $\lambda / 2$, (b) $3 \lambda / 2$, and (c) $5 \lambda / 2$ computed using NEC model in Fig. 91. . . . . . . .

Figure 953 -D normalized far field pattern of travelling-wave wire antenna, modeled in NEC with Wu-King loading along feedline of length $21 \lambda / 4.96$

Figure 96 3-D normalized far-field pattern illustrating superposition of fields from balanced dipole and travelling-wave wire antenna, computed

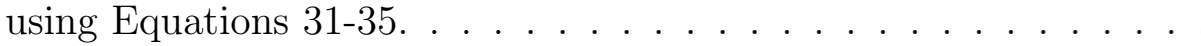

Figure 97 NEC model used to study balanced/unbalanced dipoles fed with a double-y balun in the time domain. . . . . . . . . . . . . . . . . . 101

Figure 98 Representation of NEC model in Fig. 97 as a two port network. . . 101

Figure 99 Illustration of (a) a differentiated Gaussian pulse in the time domain with pulse width $\tau_{p}$, and (b) its frequency spectrum showing spectral peak at $f_{p} \ldots \ldots \ldots \ldots \ldots \ldots \ldots \ldots \ldots \ldots \ldots \ldots \ldots$

Figure 100 Time-domain voltage received by RX dipole in Fig. 97 illustrating feedline reflections from unbalanced TX dipole. The TX dipole was excited with a differentiated Gaussian pulse with spectral peak at 3 GHz. . . . . . . . . . . . . . . . . . . . . . . . . 102

Figure 101 NEC model used to study the effect of time gating secondary reflections caused by unbalanced dipole feed on the frequency domain amplitude pattern along the azimuth plane. . . . . . . . . . . 105

Figure 102 Received time-domain voltage by RX dipole in Fig. 101, as defined in Equation 43, with an unbalanced TX dipole excited with a $1 \mathrm{~V}$-peak differentiated Gaussian pulse with spectral peak at $3 \mathrm{GHz}$. The TX dipole was modeled with a feedline length of $11 \lambda / 4$ and was rotated in increments of $5^{\circ} \ldots \ldots \ldots \ldots \ldots \ldots$

Figure 103 Time-domain voltage plots for unbalanced $5 \mathrm{~cm}$ dipole generated via NEC model in Fig. 101 at $\phi=90^{\circ} \ldots$. . . . . . . . . . . . . . . 107

Figure 104 Normalized amplitude patterns $\left(\left|\mathrm{E}_{\phi}\right|\right)$ along azimuth plane generated from NEC model in Fig. 101 at (a) $\lambda / 2$ and (b) $3 \lambda / 2$. . . . . . . 108

Figure 105 NEC model used to study the effect of time gating secondary reflections caused by unbalanced dipole feed on the frequency domain amplitude pattern along the elevation plane. . . . . . . . . . . . 109 
Figure 106 Received time-domain voltage by RX dipole in Fig. 105, as defined in Equation 43, with an unbalanced TX dipole excited with a $1 \mathrm{~V}$-peak differentiated Gaussian pulse with spectral peak at $3 \mathrm{GHz}$. The TX dipole was modeled with a feedline length of $11 \lambda / 4$ and was rotated

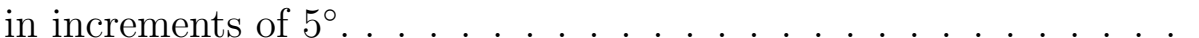

Figure 107 Time-domain voltage plots for unbalanced $5 \mathrm{~cm}$ dipole generated via NEC model in Fig. 105 at $\theta=0^{\circ}$. . . . . . . . . . . . . . . 111

Figure 108 Normalized amplitude patterns $\left(\left|\mathrm{E}_{\theta}\right|\right)$ along elevation plane generated from NEC model in Fig. 105 at $\lambda / 2$. . . . . . . . . . . . . 111

Figure 109 NEC model used to study the cross-polarized component of the radiated field from an unbalanced dipole. . . . . . . . . . . . . 115

Figure 110 Received time-domain voltage by RX dipole in Fig. 109, as defined in Equation 43, with a balanced TX dipole excited with a $1 \mathrm{~V}$-peak differentiated Gaussian pulse with spectral peak at $3 \mathrm{GHz}$. The TX dipole was modeled with a feedline length of $11 \lambda / 4$ and was rotated in increments of $5^{\circ} \ldots . . \ldots$. . . . . . . . . . . . . .

Figure 111 Received time-domain voltage by RX dipole in Fig. 109, as defined in Equation 43, with an unbalanced TX dipole excited with a $1 \mathrm{~V}$-peak differentiated Gaussian pulse with spectral peak at $3 \mathrm{GHz}$. The TX dipole was modeled with a feedline length of $11 \lambda / 4$ and was rotated in increments of $5^{\circ} \ldots \ldots \ldots$. . . . . . . . . . . . . .

Figure 112 Received time-domain voltage by RX dipole in Fig. 109, as defined in Equation 43, with an unbalanced TX dipole excited with a $1 \mathrm{~V}$ peak differentiated Gaussian pulse with spectral peak at $3 \mathrm{GHz}$. The TX was rotated in increments of $5^{\circ}$ with $\mathrm{Wu}$-King loading along the feedline. . . . . . . . . . . . . . . . .

Figure 113 Expanded view of time-domain patterns from Figs. 111 and 112, at $0^{\circ} \cdot \ldots$

Figure 114 Normalized amplitude pattern computed via NEC model in Fig. 109 for two feedline lengths. . . . . . . . . . . . . . . . .

Figure 115 Effect of common-mode currents in an antenna measurement setup.

Figure 116 New experimental setup with optical link replacing long coaxial feedline. . . . . . . . . . . . . . . . . . . . 121

Figure 117 Laser modulator unit. . . . . . . . . . . . . . . . . . . . 123

Figure 118 Alcatel 1916LMM laser module with integrated electro-absorption modulator. . . . . . . . . . . . . . . 123

Figure 119 Bias protection circuit for laser diode. . . . . . . . . . . . . . . 123 
Figure 120 Illustration of laser receiver (LR) unit (a) with enclosed LiPoly battery and (b) with battery back removed to view internal circuitry. . 125

Figure 121 Alcatel 1915DMO receiver module. . . . . . . . . . . . . . . . . . 125

Figure 122 System level diagram of receiver circuitry. . . . . . . . . . . . 126

Figure 123 Operating circuit and pinout of (a) Max1665V and (b) IRF9556 ICs. 126

Figure 124 Battery monitoring circuitry. . . . . . . . . . . . . . . 127

Figure 125 Illustration of (a) expanded view of differential output pins from 1915 module and (b) dimensions of output pins. . . . . . . . . . . 127

Figure 126 Design of RF traces for output from 1915DMO module using ADS. 129

Figure 127 Insertion loss of microstrip section obtained via ADS model illustrated in Fig. 126. . . . . . . . . . . . . . . . . . . . . 130

Figure 128 ERA-1SM RF amplifier biasing scheme. . . . . . . . . . . . . 130

Figure 129 Measured output power from LR unit. . . . . . . . . . . . . . . 131

Figure 130 Illustration of double-y balun designed with (a) 110 mil stubs and (b) 80 mil stubs. . . . . . . . . . . . . . . . . . 135

Figure 131 Expanded view of double-y balun junction illustrating through plated via holes for CPW bridges. . . . . . . . . . . . . . . . 135

Figure 132 Expanded view of coaxial line soldered to the $50 \Omega \mathrm{CPW}$ section. . 136

Figure 133 Bottom view of double-y balun with additional CPW bridges. . . . 136

Figure 134 Expanded view of two $100 \Omega$ series load resistors soldered to the CPS section for VSWR measurement. . . . . . . . . . . . . 136

Figure 135 Measured VSWR of double-y balun with 80 mil stubs and $200 \Omega$ load resistor.

Figure 136 Time-domain reflected voltage of the double-y balun with 80 mil stubs when fed with a $1 \mathrm{~V}$-peak input differentiated Gaussian pulse with spectral peak at $6 \mathrm{GHz} . \ldots \ldots \ldots \ldots$

Figure 137 Measured VSWR of double-y balun with 110 mil stubs and $200 \Omega$ load resistor. . . . . . . . . . . . . . . . 138

Figure 138 Time-domain reflected voltage of the double-y balun with 110 mil stubs when fed with a $1 \mathrm{~V}$-peak input differentiated Gaussian pulse with spectral peak at $6 \mathrm{GHz} . \ldots \ldots \ldots . \ldots 138$

Figure 139 Measured insertion loss of double-y balun with 110 mil stubs with and without additional CPW bridges. . . . . . . . . . . 139 
Figure 140 Measured insertion loss of double-y balun with 80 and 110 mil stubs. 139

Figure 141 Measured insertion loss of double-y balun (80 mil stubs) without additional CPW bridges, one additional CPW bridge, and two additional CPW bridges. . . . . . . . . . . . . . . . . 140

Figure 142 Block diagram illustrating measurement setup constructed for conducting antenna pattern measurements. . . . . . . . . . . . . . 141

Figure 143 Alignment of AUT with rotary positioner and reference probe. . . . 142

Figure 144 Illustration of AUT on rotary positioner. . . . . . . . . . . . . . . . 142

Figure 145 Illustration of (a) AUT on rotary positioner covered with microwave absorber and (b) probe antenna embedded in microwave absorber. .

Figure 146 Illustration of $5 \mathrm{~cm}$ dipole fed directly with a coaxial line, without a balun. . . . . . . . . . . . . . . . . . 145

Figure 147 Measured vs. NEC (NEC model in Fig. 76 with $\alpha=0.1$ for unbalanced and $\alpha=0.5$ for balanced dipole) azimuth amplitude patterns for unbalanced $5 \mathrm{~cm}$ dipole at (a) $\lambda / 2$ and (b) $3 \lambda / 2$. . . . . .

Figure 148 Time-domain pattern of the measured voltage at the probe with an incident double differentiated Gaussian pulse (spectral peak at $3 \mathrm{GHz}$ ) transmitted by a $5 \mathrm{~cm}$ dipole fed without a balun. . . . .

Figure 149 Plot comparing time-domain waveforms from Fig. 148 at $70^{\circ}$ and $110^{\circ} \ldots \ldots \ldots \ldots \ldots \ldots \ldots$

Figure 150 Measured vs. NEC (NEC model in Fig. 76 with $\alpha=0.5$ for balanced dipole) azimuth amplitude patterns for $5 \mathrm{~cm}$ dipole fed with double-y

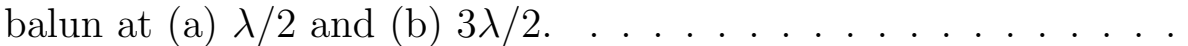

Figure 151 Time-domain pattern of the measured voltage at the probe with an incident double differentiated Gaussian pulse (spectral peak at $3 \mathrm{GHz}$ ) transmitted by a $5 \mathrm{~cm}$ dipole fed with the double-y balun. .

Figure 152 Illustration of $5 \mathrm{~cm}$ dipole fed with sleeve balun. . . . . . . . . . . 150

Figure 153 Measured vs. NEC (NEC model in Fig. 76 with $\alpha=0.5$ for balanced dipole) azimuth amplitude patterns for $5 \mathrm{~cm}$ dipole fed with sleeve balun at (a) $\lambda / 2$ and $($ b) $3 \lambda / 2 \ldots \ldots \ldots$

Figure 154 Time-domain pattern of the measured voltage at the probe with an incident double differentiated Gaussian pulse (spectral peak at $3 \mathrm{GHz}$ ) transmitted by a $5 \mathrm{~cm}$ dipole fed with the sleeve balun. . . .

Figure 155 Time-domain plot of the measured voltage at the probe with an incident double differentiated Gaussian pulse (spectral peak at $3 \mathrm{GHz}$ ) transmitted by a $5 \mathrm{~cm}$ dipole along $\phi=0^{\circ}$. 
Figure 156 Illustration of $12 \mathrm{~cm}$ dipole fed directly with a coaxial line, without a balun. . . . . . . . . . . . . . . . . 155

Figure 157 Measured vs. NEC (NEC model in Fig. 76 with $\alpha=0.1$ for unbalanced and $\alpha=0.5$ for balanced dipole) azimuth amplitude patterns for $12 \mathrm{~cm}$ dipole fed without a balun at (a) $\lambda / 2$, (b) $3 \lambda / 2$, and (c) $5 \lambda / 2 \ldots \ldots \ldots \ldots \ldots \ldots$

Figure 157 (Cont'd.) Measured vs. NEC (NEC model in Fig. 76 with $\alpha=0.1$ for unbalanced and $\alpha=0.5$ for balanced dipole) azimuth amplitude patterns for $12 \mathrm{~cm}$ dipole fed without a balun at (a) $\lambda / 2$, (b) $3 \lambda / 2$, and $(\mathrm{c}) 5 \lambda / 2 \ldots \ldots \ldots \ldots \ldots$

Figure 158 Time-domain plot of the measured voltage at the probe with an incident double differentiated Gaussian pulse (spectral peak at $3 \mathrm{GHz}$ ) transmitted by a $12 \mathrm{~cm}$ dipole fed without a balun. . . . . . . . . . 158

Figure 159 Measured vs. NEC (NEC model in Fig. 76 with $\alpha=0.5$ for balanced dipole) azimuth amplitude patterns for $12 \mathrm{~cm}$ dipole fed with the double-y balun at (a) $\lambda / 2$, (b) $3 \lambda / 2$, and (c) $5 \lambda / 2$. . . . . . .

Figure 159 (Cont'd.) Measured vs. NEC azimuth amplitude patterns for $12 \mathrm{~cm}$ dipole fed with the double-y balun at (a) $\lambda / 2$, (b) $3 \lambda / 2$, and (c) $5 \lambda / 2.160$

Figure 160 Time-domain plot of the measured voltage at the probe with an incident double differentiated Gaussian pulse (spectral peak at $3 \mathrm{GHz}$ ) transmitted by a $12 \mathrm{~cm}$ dipole fed with the double-y balun. . . . . 161

Figure 161 NEC model in Fig. 76 expanded to include laser receiver (LR) unit. 163

Figure 162 Block diagram of modified measurement system for conducting pattern measurements along elevation plane. . . . . . . . . . . 163

Figure 163 Illustration of (a) top view and (b) side view of AUT connected to the laser receiver unit mounted on the rotary positioner. . . . . . .

Figure 164 Illustration of laser modulator (LM) unit, placed underneath the positioner during antenna pattern measurement using the optical link detailed in Chapter 4. . . . . . . . . . . . . . . . .

Figure 165 Measured vs. NEC (NEC model in Fig. 161 with $\alpha=0.1$ for unbalanced and $\alpha=0.5$ for balanced dipole) elevation amplitude pattern for $5 \mathrm{~cm}$ dipole fed without a balun at $\lambda / 2$. . . . . . . . . . .

Figure 166 Time-domain pattern of the measured voltage at the probe with an incident double differentiated Gaussian pulse (spectral peak at $3 \mathrm{GHz}$ ) transmitted by a $5 \mathrm{~cm}$ dipole fed without a balun. . . . . . 168

Figure 167 Plot comparing time-domain waveforms from Fig. 166 at $20^{\circ}$ and $340^{\circ} \ldots \ldots \ldots \ldots 169$ 
Figure 168 Measured vs. NEC (NEC model in Fig. 161 with $\alpha=0.5$ for balanced dipole) elevation amplitude pattern for $5 \mathrm{~cm}$ dipole fed with the

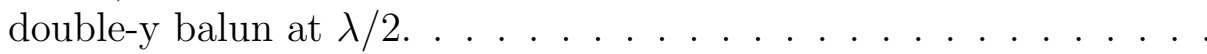

Figure 169 Time-domain pattern of the measured voltage at the probe with an incident double differentiated Gaussian pulse (spectral peak at $3 \mathrm{GHz}$ ) transmitted by a $5 \mathrm{~cm}$ dipole fed with the double-y balun. .

Figure 170 Plot comparing time-domain waveforms from Fig. 169 at $20^{\circ}$ and $340^{\circ} \ldots \ldots \ldots \ldots \ldots \ldots \ldots$

Figure 171 Measured vs. NEC (NEC model in Fig. 161 with $\alpha=0.5$ for balanced dipole) elevation amplitude pattern for $5 \mathrm{~cm}$ dipole fed with the sleeve balun at $\lambda / 2$

Figure 172 Time-domain pattern of the measured voltage at the probe with an incident double differentiated Gaussian pulse (spectral peak at $3 \mathrm{GHz}$ ) transmitted by a $5 \mathrm{~cm}$ dipole fed with the sleeve balun. . . .

Figure 173 Plot comparing time-domain waveforms from Fig. 172 at $20^{\circ}$ and

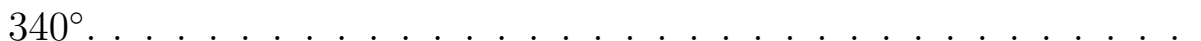

Figure 174 Measured vs. NEC (NEC model in Fig. 161 with $\alpha=0.5$ for balanced dipole) elevation amplitude pattern for $12 \mathrm{~cm}$ dipole fed without a balun at (a) $\lambda / 2$ and (b) $3 \lambda / 2 \ldots \ldots \ldots$. . . . . . . . .

Figure 175 Time-domain pattern of the measured voltage at the probe along the elevation plane, with an incident double differentiated Gaussian pulse (spectral peak at $3 \mathrm{GHz}$ ) transmitted by a $5 \mathrm{~cm}$ dipole fed without a balun. . . . . . . . . . . . . . . 176

Figure 176 Plot comparing time-domain waveforms from Fig. 175 at $30^{\circ}$ and $330^{\circ} \ldots \ldots \ldots \ldots \ldots \ldots \ldots$

Figure 177 Measured vs. NEC (NEC model in Fig. 161 with $\alpha=0.5$ for balanced dipole) elevation amplitude pattern for $12 \mathrm{~cm}$ dipole fed with the double-y balun at (a) $\lambda / 2$ and (b) $3 \lambda / 2 \ldots \ldots$. . . . . . . . . . 178

Figure 178 Time-domain pattern of the measured voltage at the probe along the elevation plane, with an incident double differentiated Gaussian pulse (spectral peak at $3 \mathrm{GHz}$ ) transmitted by a $5 \mathrm{~cm}$ dipole fed with the double-y balun. . . . . . . . . . . . . . . . . .

Figure 179 Plot comparing time-domain waveforms from Fig. 178 at $30^{\circ}$ and $330^{\circ}$.

Figure 180 Illustration of resistively loaded V-dipole fed (a) without a balun and (b) expanded view of feedpoint. 
Figure 181 Illustration of resistively loaded V-dipole fed (a) with the double-y balun and (b) expanded view of feedpoint. . . . . . . . . . . 186

Figure 182 Illustration of experimental setup for measuring the antenna pattern along the E-plane of a resistively loaded V-dipole, fed with and without the double-y balun.

Figure 183 Measured vs. numerical normalized amplitude pattern at $1 \mathrm{GHz}$ for resistively loaded V-dipole fed (a) without a balun and (b) with the double-y balun. Numerical patterns model a balanced V-dipole. . .

Figure 184 Measured vs. numerical normalized amplitude pattern at $2 \mathrm{GHz}$ for resistively loaded V-dipole fed (a) without a balun and (b) with the double-y balun. Numerical patterns model a balanced V-dipole. . .

Figure 185 Measured vs. numerical normalized amplitude pattern at $3 \mathrm{GHz}$ for resistively loaded V-dipole fed (a) without a balun and (b) with the double-y balun. Numerical patterns model a balanced V-dipole. . .

Figure 186 Measured vs. numerical normalized amplitude pattern at $4 \mathrm{GHz}$ for resistively loaded V-dipole fed (a) without a balun and (b) with the double-y balun. Numerical patterns model a balanced V-dipole. . .

Figure 187 Measured vs. numerical normalized amplitude pattern at $5 \mathrm{GHz}$ for resistively loaded V-dipole fed (a) without a balun and (b) with the double-y balun. Numerical patterns model a balanced V-dipole. . .

Figure 188 Measured time-domain pattern of received voltage by the RX Vdipole in Fig. 182, with the transmitting TX V-dipole fed without the double-y balun (illustrated in Fig. 180). The TX V-dipole was excited with a $1 \mathrm{~V}$-peak differentiated Gaussian pulse with spectral peak at $3 \mathrm{GHz} \ldots \ldots \ldots \ldots \ldots \ldots \ldots$

Figure 189 Measured time-domain pattern of received voltage by the RX Vdipole in Fig. 182, with the transmitting TX V-dipole fed with the double-y balun (illustrated in Fig. 181). The TX V-dipole was excited with a $1 \mathrm{~V}$-peak differentiated Gaussian pulse with spectral peak at $3 \mathrm{GHz} . \ldots . \cdots \cdots$

Figure 190 Plots comparing time-domain waveforms in Fig. 189 of the received voltage by the RX V-dipole at $60^{\circ}$ and $300^{\circ}$, with the TX V-dipole fed (a) without a balun and (b) with the double-y balun. . . . . . .

Figure 191 Comparison of the time-domain received voltage by the RX V-dipole (in Fig. 182) at $0^{\circ}$, with the TX V-dipole fed (a) without and (b) with the double-y balun. The TX V-dipole was excited with a 1 V-peak differentiated Gaussian pulse with spectral peak at $3 \mathrm{GHz}$. 
Figure 192 Plot of relative gain, as defined in Equation 44, with RX and TX V-dipoles facing each other at $0^{\circ} \ldots \ldots \ldots \ldots$

Figure 193 Illustration of 2-port network. . . . . . . . . . . . . . . . . . 203 


\section{SUMMARY}

In this research, a double-y balun implemented with coplanar waveguide (CPW) and coplanar strip (CPS) is investigated for use with pulsed antennas. The balun is modeled using two commercial electromagnetic simulators: Momentum and HFSS. Using these numerical solvers, design information such as the design of CPW bridges, aspect ratio of the double-y balun, and stub lengths of the CPW and CPS open and short stubs are studied.

A dipole, along with the outer conductor of a coaxial line is modeled in NEC. The NEC model serves two purposes: (1) provide better insight into the effects of an unbalanced dipole feed and (2) generate antenna patterns that serve as a basis to which measured data can be compared. The model is used to study the currents along the arms and feedline of balanced and unbalanced dipoles. Normalized amplitude patterns are generated along the azimuth and elevation planes for balanced and unbalanced dipoles. These patterns are used later for comparison with measured patterns.

Experimental work is conducted to measure the performance of a double-y balun designed to feed a resistively loaded V-dipole. The performance of the balun is investigated via VSWR, insertion loss, and antenna pattern measurements. Antenna pattern measurements along the azimuth plane are conducted for a $5 \mathrm{~cm}$ dipole fed without a balun, a $5 \mathrm{~cm}$ dipole fed with the double-y balun, a $5 \mathrm{~cm}$ dipole fed with the sleeve balun, a $12 \mathrm{~cm}$ dipole fed without a balun, and a $12 \mathrm{~cm}$ dipole fed with the double-y balun. The dipoles fed without a balun were fed directly with a $50 \Omega$ coaxial line. An optical link, consisting of a laser modulator (LM) unit and a laser receiver (LR) unit, was constructed to measure the patterns along the elevation plane 
of the above dipoles. Resulting patterns agree closely with patterns generated with NEC models. In addition, the patterns of a resistively loaded V-dipole are measured along the E-plane using the optical link. The measured patterns are compared with numerical results obtained from literature. The experimental work conducted in this research illustrates the improvement obtained in the patterns of a dipole and a resistively loaded V-dipole with the use of the double-y balun. 


\section{CHAPTER I}

\section{INTRODUCTION}

Many popular antennas, such as the dipole, the complementary spiral, and the resistively loaded V-dipole, require a balanced feed to radiate properly. A balanced feed is defined as having only differential currents (equal in magnitude and opposite in direction) along the feedline of the antenna. This is illustrated in Fig. 1, where the conductors of a two-wire transmission line are flared to form a dipole. The currents along the transmission line (feedline) are differential. The desired radiation patterns of the above mentioned antennas are computed with the assumption that the antenna feedline is balanced.

A coaxial line, illustrated in Fig. 2, is often employed to carry high-frequency signals. The coaxial line consists of an inner conductor, surrounded by a dielectric and an outer conductor. In this work, the coaxial line is defined as being asymmetric; while the coaxial line does exhibit certain symmetry in cylindrical coordinates, it is defined as being asymmetric in the sense that the physical dimensions of its conductors are not equal. That is, we cannot transform one (e.g. inner conductor) conductor into the other (e.g. outer conductor) conductor via rotation. Some transmission lines, such as the two-wire transmission line, are symmetric. One conductor of the two-wire transmission line can be formed by rotating the other conductor about an axis, since both conductors have equal physical dimensions. Figure 3 compares a coaxial line and a two-wire transmission line to better illustrate the difference between an asymmetric structure and a symmetric structure, as defined in this work.

Figure 4 illustrates a dipole (requires a balanced feed) fed directly by a coaxial line. 


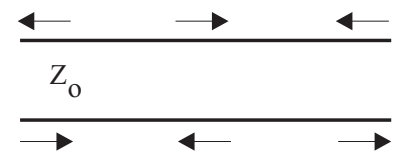

(a)

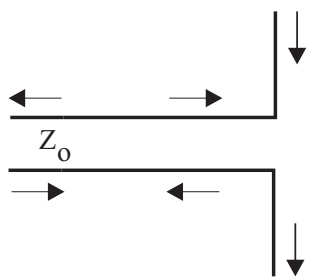

(b)

Figure 1: Illustration of differential currents along (a) a two-wire transmission line and (b) a dipole fed by a two-wire transmission line.

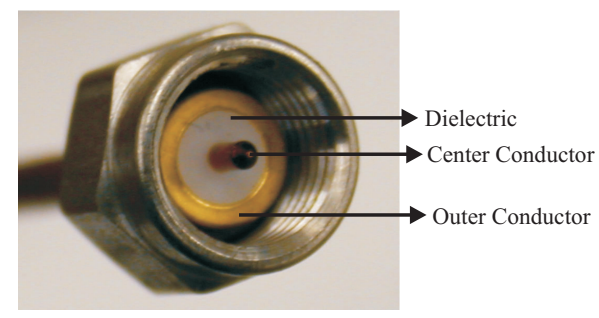

Figure 2: Illustration of a coaxial line.

It is seen that in addition to the balanced signal currents $I_{1}$ and $I_{2}$, a net commonmode current (labeled $\mathrm{I}_{3}$ in Fig. 4) is induced along the the coaxial line. The commonmode current is induced because of the asymmetrical coupling between the arms of the antenna and the conductors of the coaxial line. This common-mode current causes the arms of the antenna to become unbalanced, resulting in radiation from the feedline and distortion of the desired radiation pattern of the antenna. Because of the asymmetry between its conductors, the coaxial line is referred to as an unbalanced structure. In contrast, the conductors of a balanced structure exhibit symmetry, as defined previously and illustrated in Fig. 3. It is important to distinguish between the terms balanced feed, defined earlier, and balanced structure. While a balanced feed is defined as has having only differential-mode currents, balanced and unbalanced structures can support both, common-mode and differential-mode currents.

To properly transition from an unbalanced structure (e.g. coaxial line) to a balanced structure (e.g. symmetric antenna), a balun is required. The fundamental purpose of a balun is to prevent the common-mode current, $\mathrm{I}_{3}$ in Fig. 4, from being 


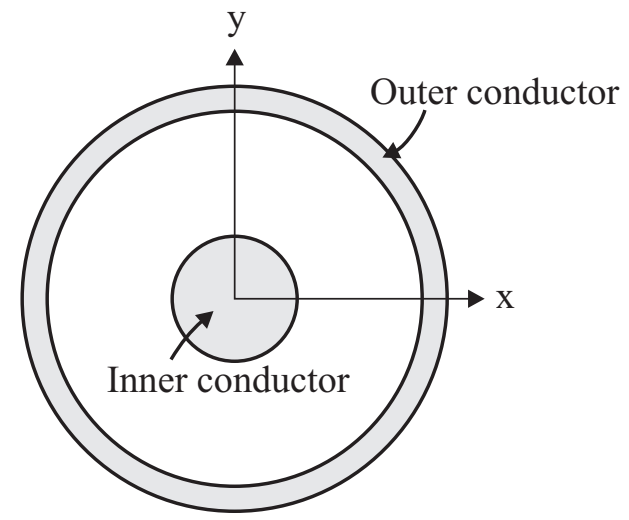

Note: Outer conductor cannot be constructed by rotating inner conductor

(a)

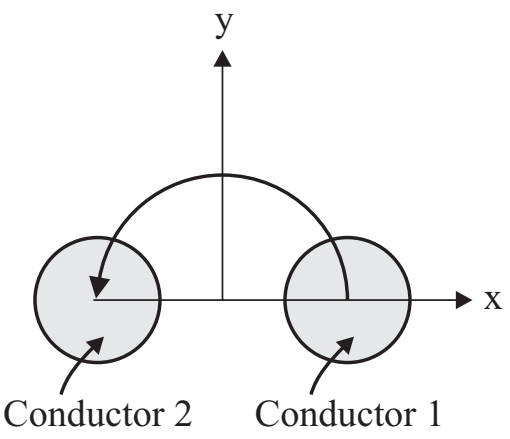

Note: Conductor 2 can be obtained by rotating Conductor 1

(b)

Figure 3: Cross section of (a) asymmetric coaxial line and (b) symmetric two-wire transmission line. The coaxial line is defined as an unbalanced structure, and the two-wire transmission line is defined as a balanced structure. 


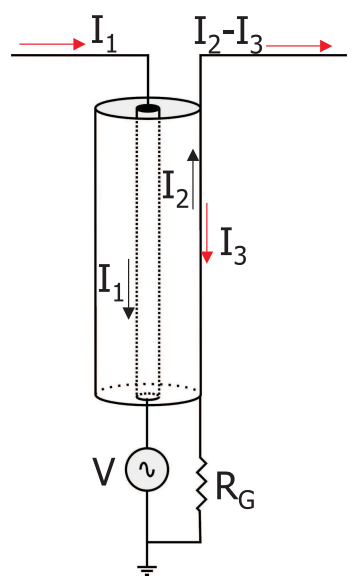

Figure 4: Illustration of dipole fed directly by a coaxial line.

induced along the coaxial line, thus providing a balanced feed for the balanced structure, such as a symmetric antenna. In some cases there is an impedance mismatch between the balanced and unbalanced structures, and it is desirable for the balun to provide inherent impedance transformation capability. If the balun cannot provide inherent impedance transformation, an external matching network is required to provide the necessary impedance transformation. Several popular types of baluns are discussed next to illustrate the variety of approaches that been devised to properly transition from an unbalanced structure to a balanced structure.

One type of balun is constructed, as illustrated in Figure 5, by slowly peeling the outer conductor of a coaxial cable in a tapered fashion until it becomes a two-wire transmission line $[4,5]$. The total current on the outer surface of the coaxial line results from the summation of wave reflections originating over the entire length of the transition. The transition is therefore tapered as to minimize these reflections. Thus, negligible current appears on the outside surface of the coaxial line and electrical balance is achieved at the two-wire transmission line section. Impedance transformation can be achieved via the gradual tapering of the coaxial line into a two-wire transmission line. In [4], a 50:1 bandwidth was achieved with a VSWR of less than 1.25. Although large operating bandwidths can be achieved by this balun, the overall 
length is limited to at least $\lambda / 2$ at the lowest frequency, thus making this balun very long for some practical applications.

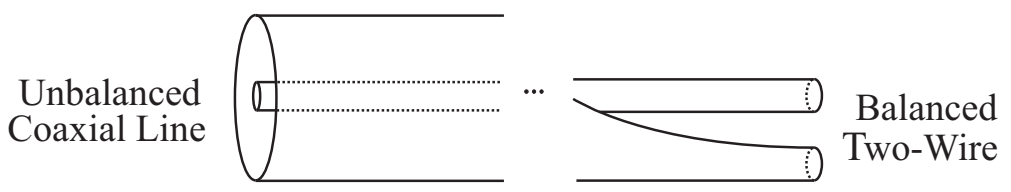

Figure 5: Balun constructed by tapering outer conductor of coaxial cable.

The Guanella balun, or the transmission-line balun, is illustrated in Figure 6 . The balun consists of a bifilar winding around a magnetic core, connected such that differential currents are allowed to flow, while common-mode currents are choked $[3,6$, 7]. Figure 7 illustrates an equivalent circuit for analyzing the Guanella balun, where $Z_{S}$ is the source impedance, $Z_{L O A D}$ is the load impedance, $Z_{G}$ is a finite impedance to ground, $Z_{C M}$ is the common-mode impedance, $X_{L}$ is the reactance of the windings, $M$ is the mutual inductance between the windings, and currents $I_{D}$ and $I_{C M}$ are the differential and common-mode currents, respectively. The common-mode current, $I_{C M}$, is given by

$$
I_{C M}=\frac{Z_{G}}{Z_{G}+Z_{C M}+X_{L}} I_{D}
$$

where it has been assumed that the self and mutual inductances of the coils are equal $[7,8]$. This assumption is valid if both windings are identical and are closely coupled to the core. From Equation 1, it is seen that the common-mode current can be attenuated by either increasing the common-mode impedance or by the choking action of the windings (the self-inductance of the coils must be large compared to $\left.Z_{G}+Z_{C M}\right)$. At high frequencies, the distributed capacitance of the windings must be included in the circuit model. This capacitance limits the high-frequency operation of the balun [8]. While the Guanella balun does not provide inherent impedance transformation capability, variations such as the Ruthroff balun in [9] can achieve impedance transformation.

Baluns have also been constructed by loading the outer conductor of the coaxial 


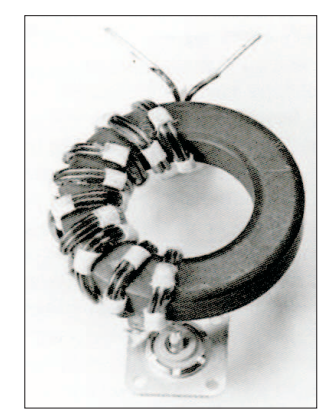

Figure 6: Illustration of the Guanella balun [3].

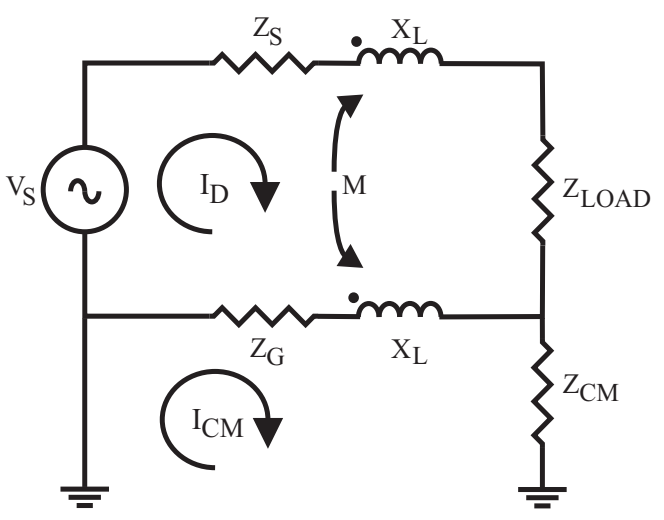

Figure 7: Equivalent lumped element model of the Guanella balun.

line with ferrite beads $[8,10,11]$. Such a balun is illustrated in Figure 8, where it is feeding a dipole. The inductance of the ferrite beads chokes the common-mode current on the outside surface of the coaxial line. This is illustrated in Figure 9a, where it is seen that a time-varying common-mode current induces a magnetic flux in the ferrite bead's core, causing the bead to behave inductively. The ferrite beads have no effect on differential-mode current. This is illustrated in Figure 9b, where the magnetic flux resulting from the differential-mode currents cancel in the core. Advantages for this type of balun when compared to a bifilar current balun wound on a ferrite toroid include superior impedance-versus-frequency characteristics and excellent power-handling capability. In addition, no core saturation occurs at high power levels in the ferrite beads since the core excitation is produced by only the current on the outside surface of the coaxial line. However, the beads can get extremely hot and their use is limited to frequencies below $1 \mathrm{GHz}$ [11]. Manufacturers typically 
characterize the beads by specifying the magnitude of the impedance as a function of frequency. The magnitude of the bead impedance is given by

$$
Z_{S B}=\sqrt{R^{2}+(2 * \pi f \omega L)^{2}}
$$

where $R$ is the equivalent resistance of the bead and $L$ is the equivalent inductance [8].

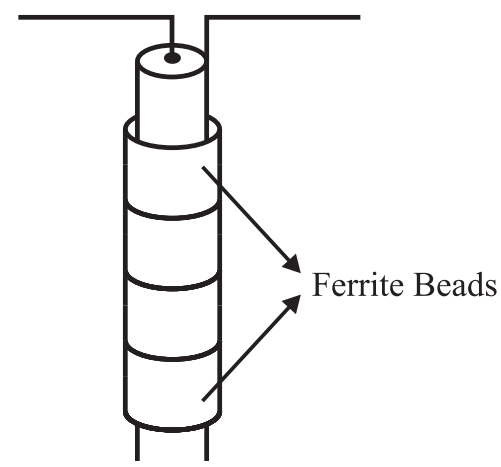

Figure 8: Illustration of coaxial line loaded with ferrite beads and feeding dipole.

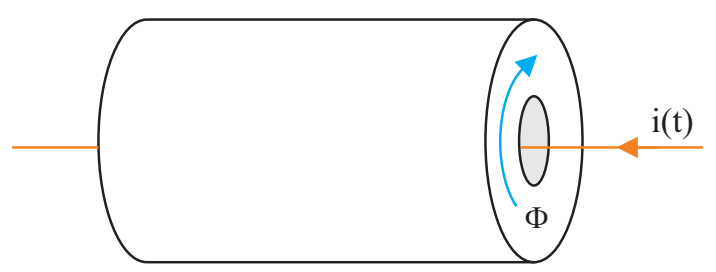

(a)

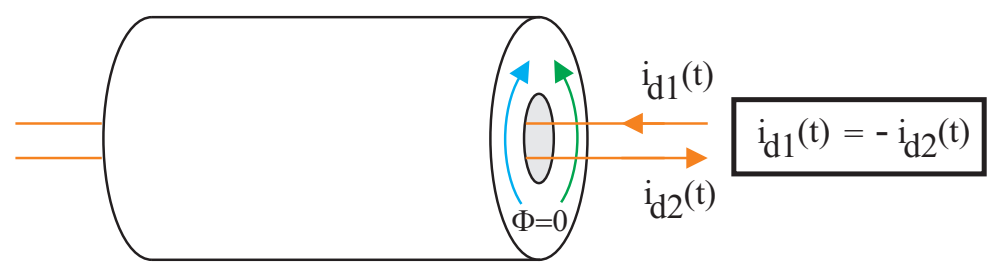

(b)

Figure 9: Magnetic flux induced in ferrite bead due to (a) common-mode and (b) differential-mode currents.

Figure 10 illustrates a balun that employs the use of phase shifting in order to achieve a balanced output. The half-wavelength coaxial stub provides a phase shift of $180^{\circ}$ between the balanced ports. However, like the sleeve balun, this balun is narrowband because of the half-wavelength section. Planar versions of this balun have 
been designed with increased bandwidth by cascading quarter-wavelength and halfwavelength sections [12]. This type of balun achieves a 4:1 balanced-to-unbalanced impedance transformation ratio [13]. Therefore, this balun can be used to match a $50 \Omega$ unbalanced coaxial line to a $200 \Omega$ antenna.

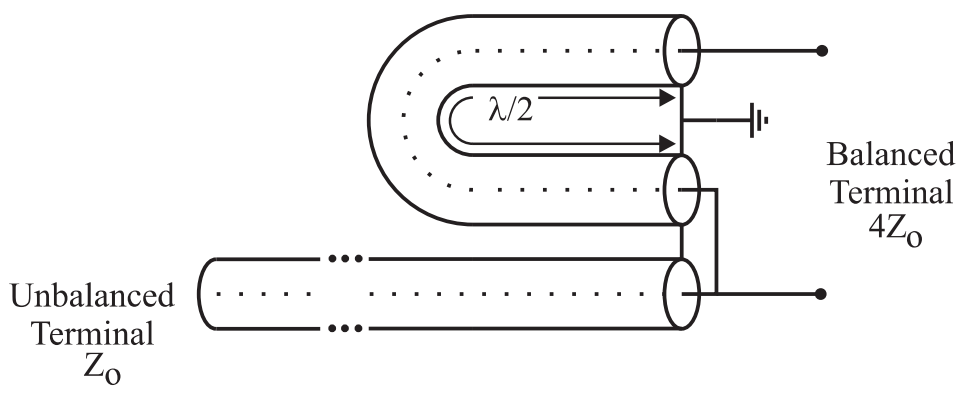

Figure 10: Balun employing phase shift to achieve balancing.

The sleeve balun, also known as the bazooka balun, is often used for feeding dipoles. In this research, a sleeve balun is constructed and antenna patterns are measured for a dipole fed with the balun. The resulting patterns are compared with patterns obtained for a dipole fed with and without the double-y balun. Figure 11 illustrates a drawing of the sleeve balun. As the name implies, a quarter-wavelength conductive sleeve is placed around the coaxial line near the antenna feed. Hence, a transmission line mode is setup between the conductive sleeve and the outer surface of the coaxial line. The input impedance looking into this shorted section of transmission line is given by

$$
Z_{\text {short }}=j Z_{o} \tan (\beta l),
$$

where $l$ is the length of the sleeve balun, and $Z_{o}$ is the characteristic impedance of the transmission line section formed by the outer conductor of the coaxial line and the sleeve balun. At $\lambda / 4$, the input impedance looking into the transmission line is infinite, thus choking the current along the the outer surface of the coaxial line. However, the sleeve balun is a narrowband balun, as the high input impedance is achieved only at odd multiples of $\lambda / 4$. 


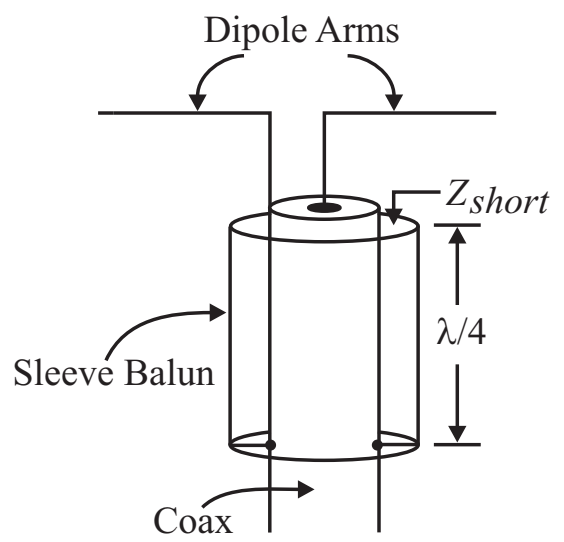

Figure 11: Illustration of the sleeve balun placed around a coaxial line feeding a dipole.

A lattice balun, implemented with lumped elements, is illustrated in Fig. 12a. Figure $12 \mathrm{~b}$ illustrates the operation of this balun. It is seen that by choosing appropriate values for the capacitors and inductors, the circuit transitions from an unbalanced structure to a balanced structure by shifting the phase of one port by $+90^{\circ}$ and the other by $-90^{\circ}$. The balun, however, is narrowband, and achieves perfect balancing at a single frequency. This balun has also been implemented with distributed elements in $[14]$.

Another type of balun is the double-y balun. Double-y baluns have been presented as a new class of miniature, wideband devices that are suitable for MMICs and wireless applications. Four different realizations of the double-y balun have been studied: (1) providing transition between microstrip-to-slotline, (2) coplanar waveguide-toslotline, (3) coplanar waveguide with finite ground plane (CPW)-to-coplanar strip (CPS), and (4) CPW-to-parallel microstrip line [15]. The balun of interest in this research is a double-y balun, implemented with coplanar waveguide (CPW) and coplanar strip (CPS) lines. The double-y balun, illustrated in Fig. 13a, transitions from a three-conductor CPW line to a two-conductor CPS line. As illustrated, the balun consists of CPW and CPS open and short stubs. Figure 13b illustrates the balun junction, where the CPW to CPS mode conversion occurs. CPW bridges (the bridges 


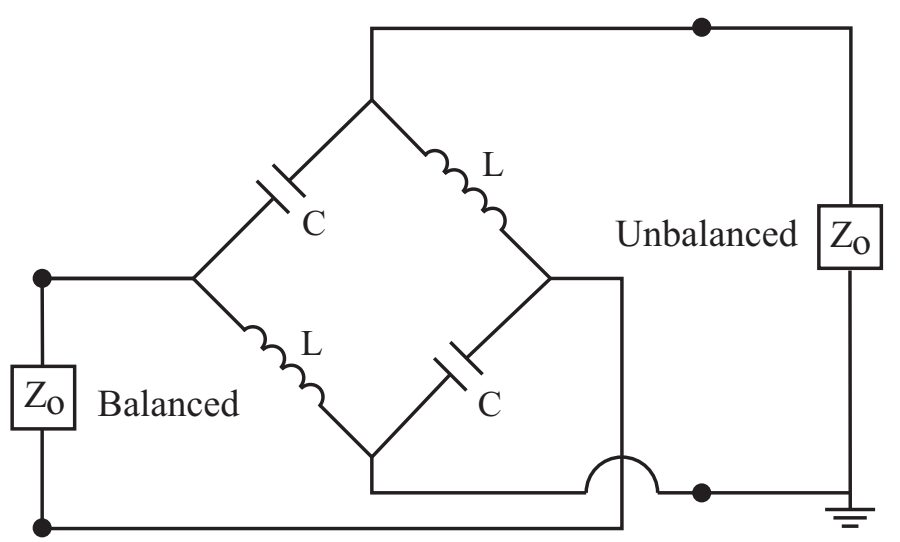

(a)

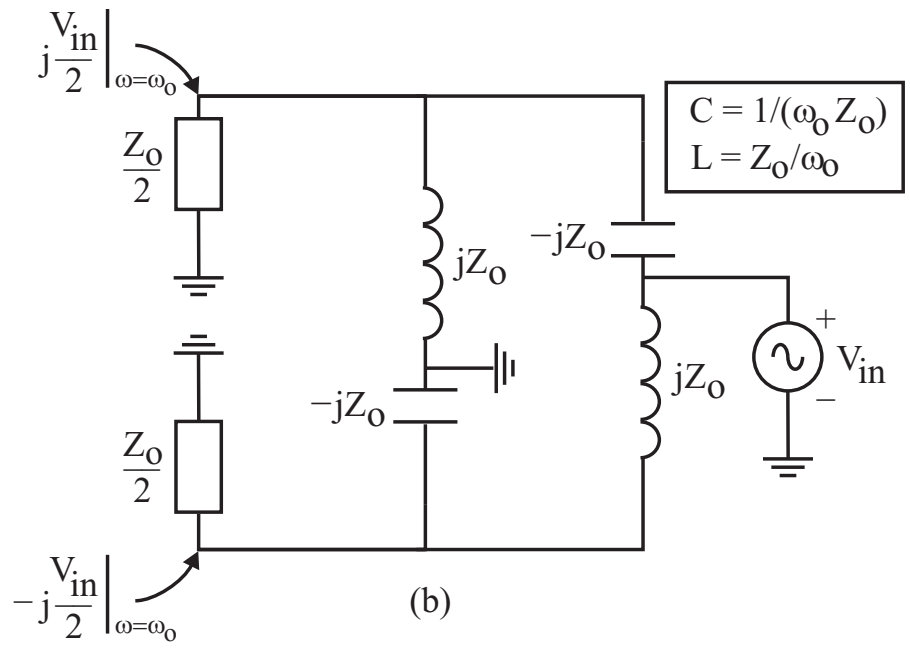

Figure 12: Illustration of (a) the lattice balun implemented with lumped element components and (b) operation of circuit as a balun. 
connect the outer ground CPW conductors) are placed at the balun junction; these bridges are necessary, as will be explained shortly, for proper operation of the balun. The lengths of the CPW and CPS open and short stubs, labeled $\mathrm{L}_{s}$ in Fig. 13c, also affect the performance of the double-y balun. Figure 13c illustrates the reference points used when measuring the lengths of the open and short stubs. Further discussion on the effects of the stub lengths is addressed in Chapter 2.

Next, the operation of the double-y balun is discussed. Figure 14a illustrates the double-y junction as a six port device. It is assumed that the reflection coefficient at ports 2, 3, 5, and 6 are equal. Furthermore, it is assumed that the electrical length of the CPW and CPS open and short stubs are equal. Parasitic effects at the double-y junction are neglected. An input signal applied at Port 1 is split between ports 2, 3, 5, and 6 with equal phase and equal amplitude. With equal reflection coefficients at ports $2,3,5$, and 6 , the signal is reflected back to Port 1, thus isolating ports 1 and 4. In Figure 14b, an input signal is applied at Port 4. Again, the input signal is split equally among ports $2,3,5$, and 6 , this time in phase opposition at ports 2 and 5 with respect to ports 3 and 6 . With equal reflection coefficients at ports $2,3,5$, and 6, the signal is reflected back to Port 4, thus isolating ports 1 and 4 . Therefore, a reflection coefficient of $\exp (\mathrm{j} \phi)$ at ports 2 and 5 and of $\exp \left(\mathrm{j} \phi+180^{\circ}\right)$ at ports 3 and 6 causes transmission between ports 1 and 4 [16]. Thus, we arrive at the configuration in Fig. 14c, where alternating open and short CPW and CPS stubs are placed at the junction to ensure transmission between ports 1 and 4 .

Figure 15a illustrates the equivalent circuit model of the double-y balun in Fig. 14c. For this model to be valid, CPW bridges are necessary at the balun junction, as illustrated in Fig. 13b. Parasitics at the balun junction are not included in this model. A more in-depth analysis is provided in Chapter 2 regarding the impedance characteristics of the double-y balun using the equivalent circuit model. At this point, it is sufficient to know that if Port 1 has a characteristic impedance of $\mathrm{Z}_{o}$, and ports 


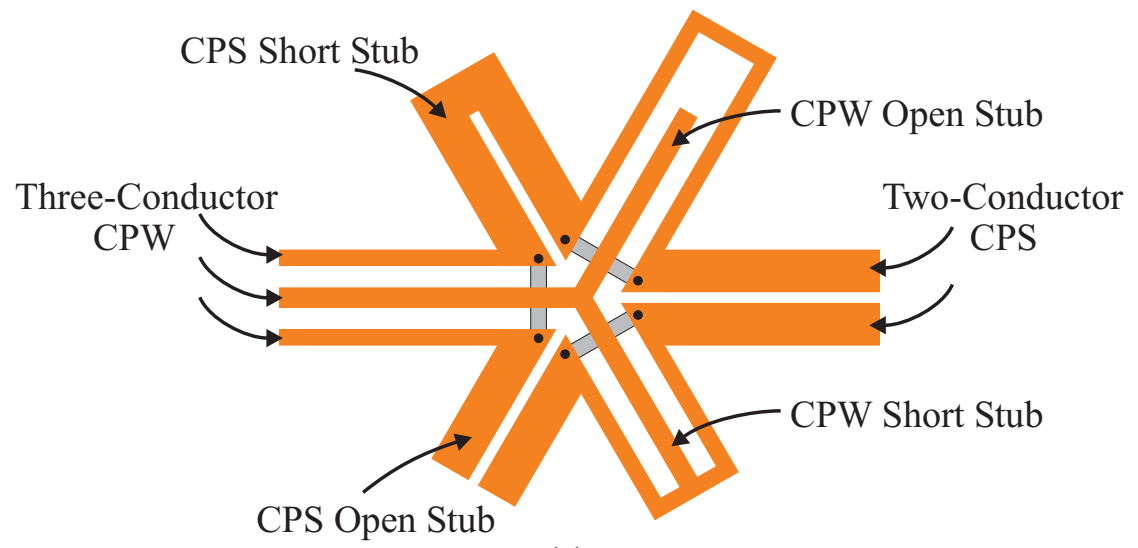

(a)

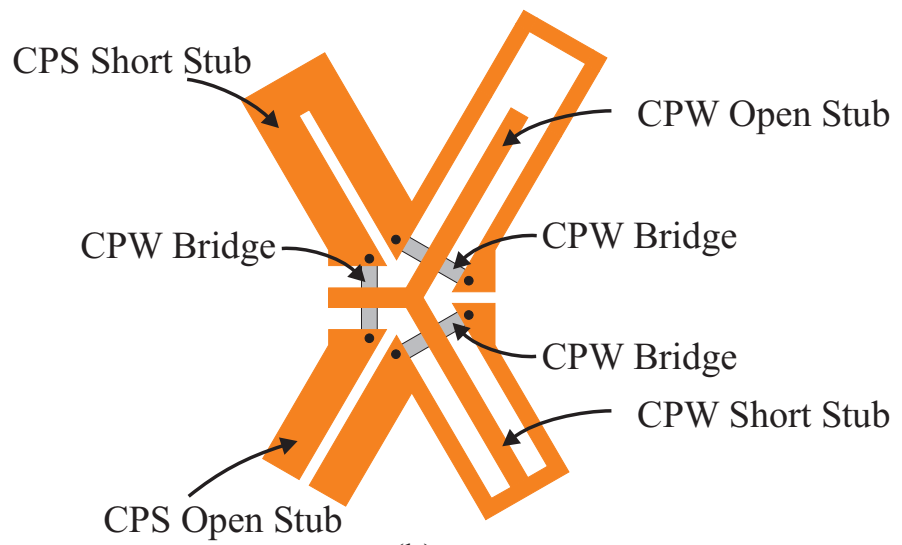

(b)
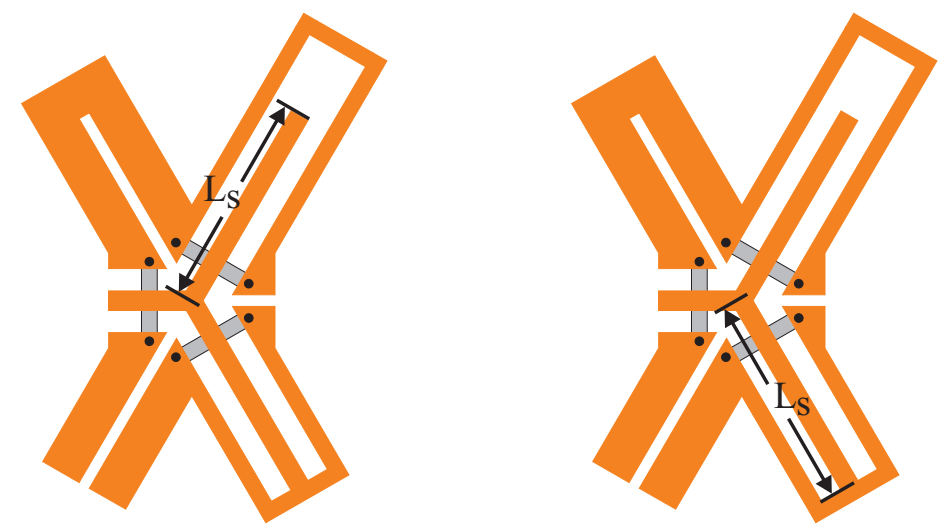

(c)

Figure 13: Illustration of (a) the double-y balun with input/output CPW and CPS lines, (b) the double-y junction where the mode conversion occurs between CPW and CPS lines, and (c) reference points used to measure the length of the CPW and CPS stubs. 


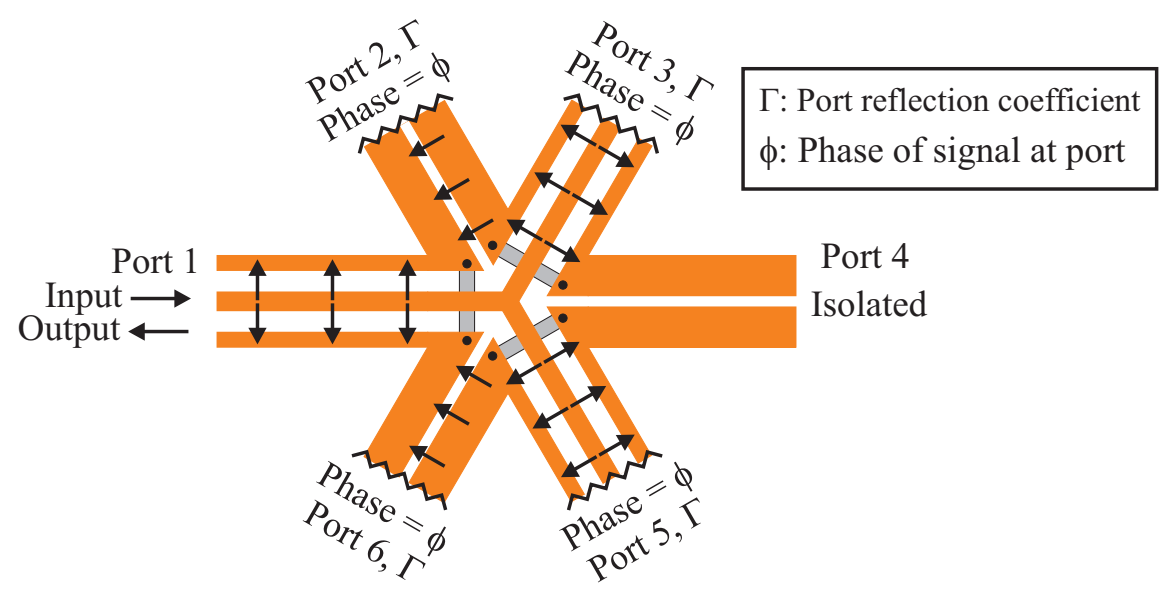

(a)

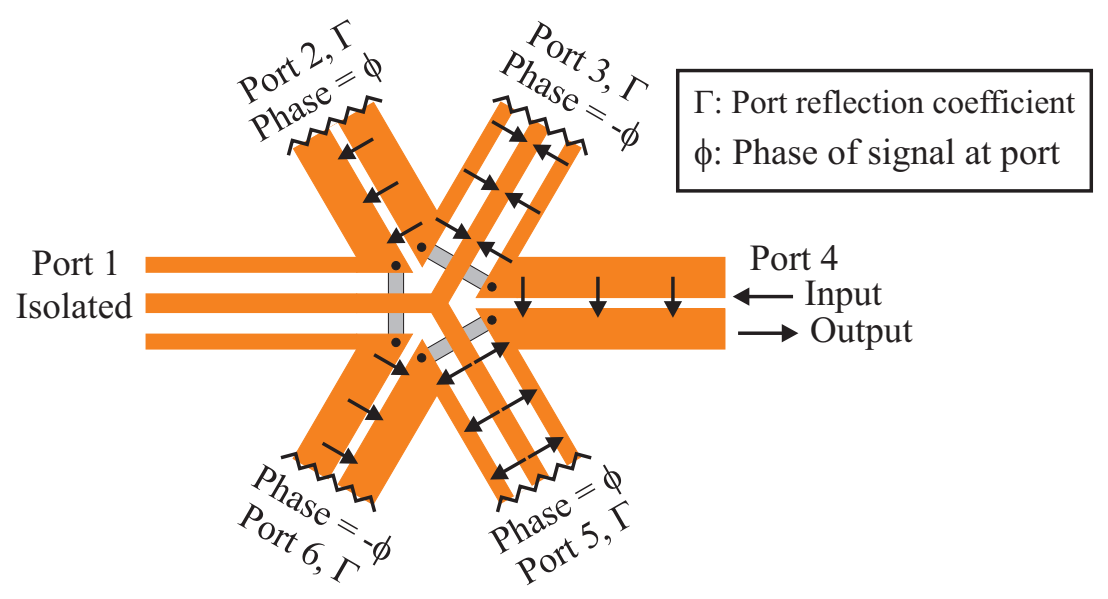

(b)

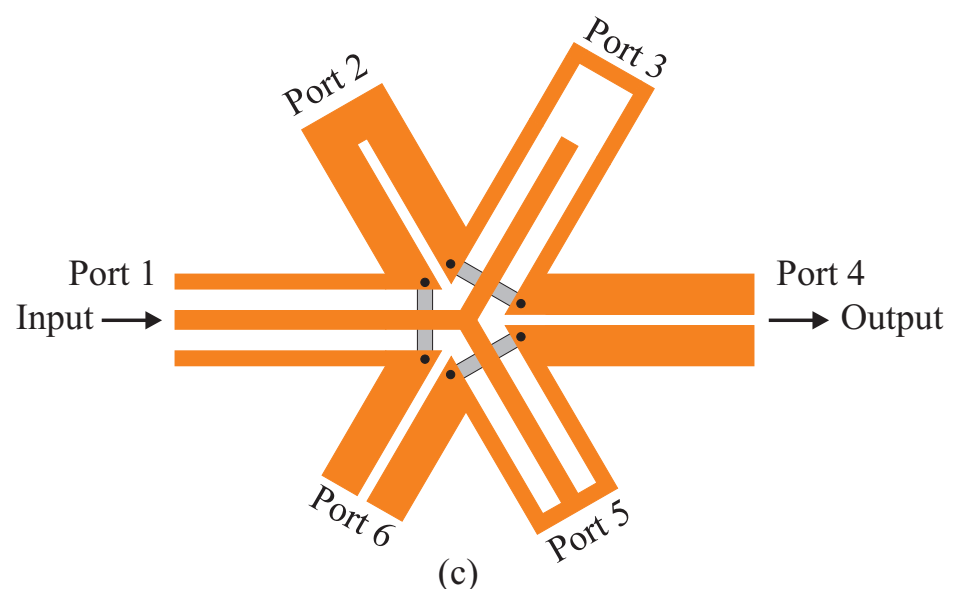

Figure 14: Illustration of (a) the double-y junction with an input signal applied to Port 1, (b) the double-y junction with an input signal applied to Port 2, and (c) the double-y balun. In (a) and (b), $\Gamma$ is the reflection coefficient at each port, and $\phi$ is the phase of the incident signal at the port. 
$2,3,5$, and 6 also have a characteristic impedance of $\mathrm{Z}_{o}$, then the balun is matched at ports 1 and 4 if Port 4 is terminated with an impedance $Z_{o}$. This assumes the electrical length of the CPW and CPS open and short stubs are equal.

Next, the balancing behavior of the double-y balun is examined. The input impedance looking into a shorted section of transmission line was previously given in Equation 3. The input impedance looking into a section of transmission line with an open load is given by

$$
Z_{\text {open }}=-j Z_{o} \cot (\beta l),
$$

where $l$ is the length of the transmission line, and $Z_{o}$ is the characteristic impedance of the transmission line. For odd multiples of $\lambda / 8$, Equations 3 and 4 reduce to $j Z_{o}$ and $-j Z_{o}$, respectively. Hence, the equivalent model in Fig. 15a can be replaced with that in Fig. 15b. The equivalent model in Fig. 15b is identical to the lattice balun, whose operation was illustrated previously in Fig. 12c. The equivalent model for the double-y balun in Fig. 15a reduces to the model in Fig. 15b for odd multiples of $\lambda / 8$. Therefore, the double-y balun achieves perfect balance at multiples of $\lambda / 8$. However, it will be shown in the next chapter that due to dispersion and parasitic effects at the balun junction, the input impedance of the double-y balun is very sensitive at $\lambda / 8$.

The double-y balun has been studied in $[17,18,19,15,20,21,22]$ for use with double-balanced mixers, yet specific design guidelines have been lacking. In this research work, the double-y balun implemented with CPW and CPS lines is investigated for feeding a resistively loaded V-dipole. Numerical analysis of the double-y balun is presented in Chapter 2. Design of CPW and CPS lines is discussed, and design curves are presented for CPW and CPS lines over FR4 substrate. Modeling of the double-y balun using two commercial electromagnetic codes, Momentum and HFSS, is presented. Both commercial simulators are used to study various design aspects of the double-y balun such as: parasitic resonances limiting the bandwidth of operation, effect of substrate thickness, aspect ratio, and stub lengths of the double-y balun. 


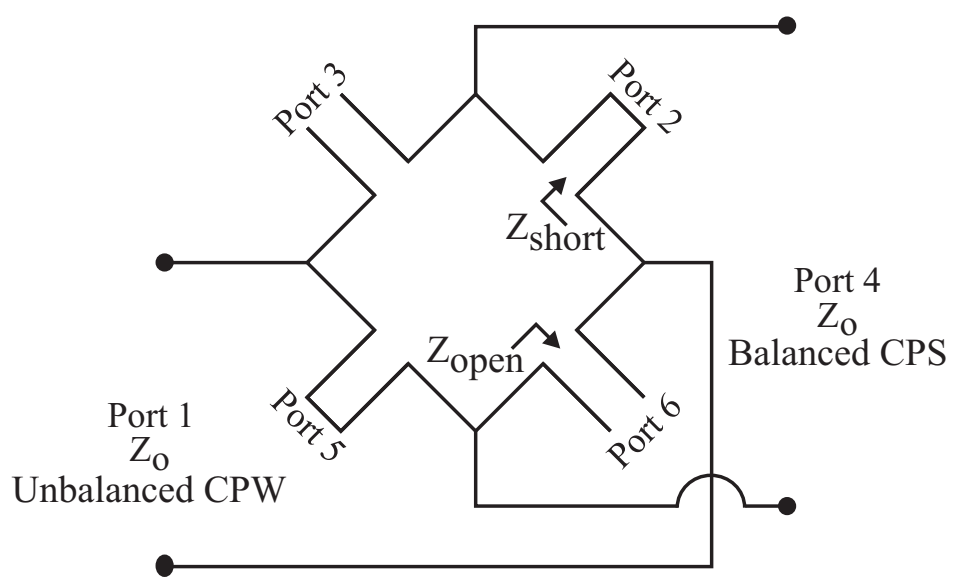

(a)

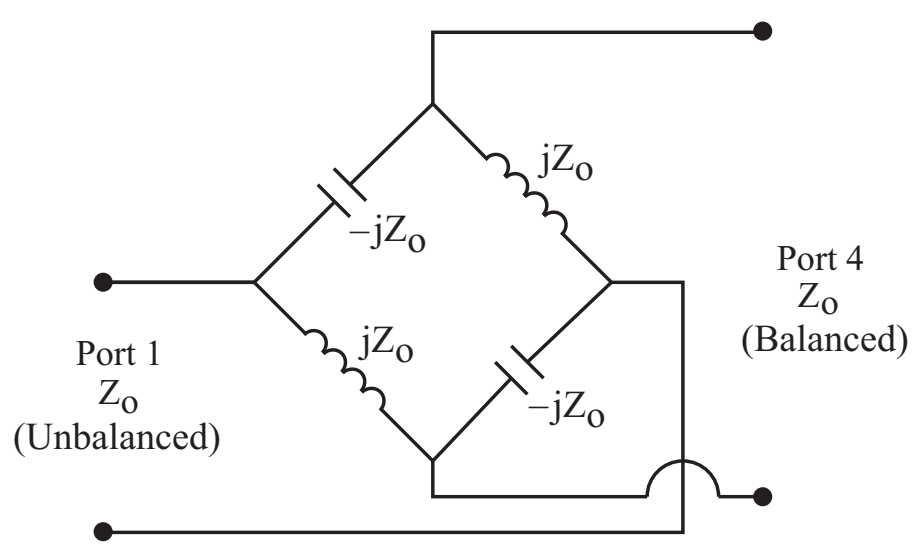

(b)

Figure 15: Illustration of (a) the equivalent circuit model of the double-y balun and (b) equivalent circuit model of double-y balun with stub lengths equal to odd multiples of $\lambda / 8$.

Having investigated various design aspects of the double-y balun, a double-y balun is designed for feeding a resistively loaded V-dipole.

In Chapter 3, a dipole along with the outer conductor of a coaxial line, is modeled in Numerical Electromagnetic Code (NEC). Due to its simple geometry, the dipole can be modeled numerically and constructed experimentally with ease. In addition, field patterns radiated by the dipole as well as current distribution along the arms of a balanced dipole are well known. Constructing a model in NEC serves two purposes: (1) provide a better understanding regarding the effects of an unbalanced feed, and 
(2) provide a basis to which measured patterns can be compared. The effect of an unbalanced dipole feed is investigated by looking at the currents along the arms and feedline of the dipole. Antenna patterns are generated in NEC for balanced and unbalanced dipoles. Also, the effects of an unbalanced feed is investigated in the time domain using NEC models of an unbalanced dipole .

Based on results obtained from the NEC model in Chapter 3, a new measurement system is constructed for measuring antenna patterns of a dipole along the elevation plane. The measurement system consists of an optical link with a laser transmitter and a laser receiver. Chapter 4 explains the purpose of the new antenna pattern measurement system, and details the design of the photonic link used in the new measurement system. Design aspects of the laser transmitter and laser receiver units are discussed in this chapter.

Chapter 5 details experimental work conducted in this research to measure the performance of the double-y balun. VSWR and insertion loss measurements are conducted on the double-y balun designed in Chapter 2 with a matched load and back to back balun configuration, respectively. Performance of the double-y balun with additional CPW bridges is also investigated via insertion loss measurements. Antenna pattern measurements are conducted for a $5 \mathrm{~cm}$ and $12 \mathrm{~cm}$ dipole fed with and without the double-y balun. In addition, antenna patterns are measured for a $5 \mathrm{~cm}$ dipole fed with a sleeve balun. For pattern measurements along the elevation plane, the new measurement system described in Chapter 4 is used. Antenna pattern measurements are compared with patterns generated via NEC in Chapter 3. The double-y balun in this research is investigated for use with pulsed antennas. Therefore, antenna pattern measurements are also conducted for a resistively loaded V-dipole fed with and without the double-y balun. Finally, conclusions stemming from this research are addressed in Chapter 6 . 


\section{CHAPTER II}

\section{DOUBLE-Y BALUN - NUMERICAL ANALYSIS}

In this chapter, numerical analysis is conducted on the double-y balun implemented with coplanar waveguide (CPW) and coplanar strip (CPS) lines. First, design charts are generated for CPW and CPS lines over FR4 substrate. Using these charts, the influence of design parameters, such as substrate thickness, gap widths, conductor widths, and substrate permittivity, on the characteristic impedance of CPW and CPS lines is studied. Conductor and dielectric loss is also studied for CPW and CPS lines over FR4 substrate. Understanding these design parameters is necessary for designing the double-y balun. A prototype double-y balun is designed for numerical analysis with two commercial electromagnetic solvers: Momentum and HFSS. Various aspects of the double-y balun are studied using both commercial simulators. Using numerical results, a new double-y balun is designed and manufactured.

\subsection{CPW/CPS Lines}

Coplanar waveguide (CPW) and coplanar strip (CPS) lines find wide use in microwave applications. The CPW is a three conductor structure (sometimes a ground plane is also added below the substrate), while the CPS is a two conductor structure, both are illustrated in Figs. 16 and 17. The double-y balun of interest in this research transitions from an unbalanced CPW structure to a balanced CPS structure, as illustrated in Fig. 18. Therefore, the synthesis of CPW and CPS lines is studied in this section. Impedance curves are generated for CPW and CPS lines over FR4 substrate $\left(\varepsilon_{r}=4.4, \delta_{t a n}=0.012\right)$. The effect of substrate thickness, gap widths, conductor widths, and substrate permittivity on the characteristic impedance of CPW and CPS 


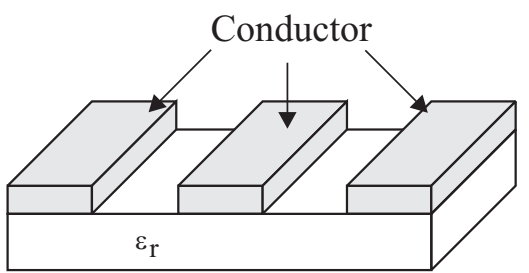

(a)

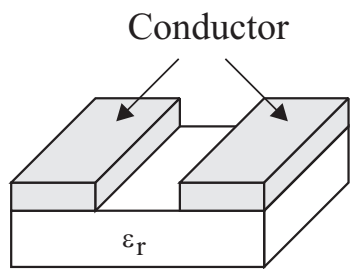

(b)

Figure 16: Illustration of (a) CPW and (b) CPS lines.

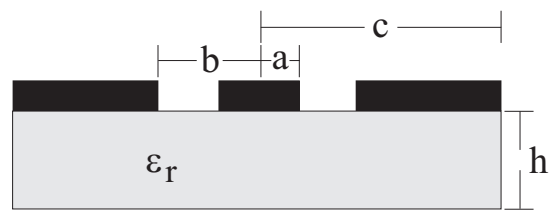

(a)

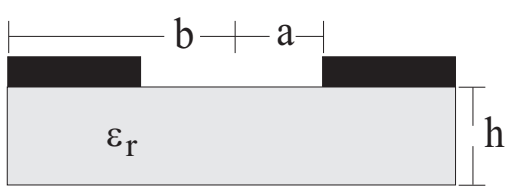

(b)

Figure 17: Cross-section view of (a) CPW and (b) CPS lines.

lines is investigated.

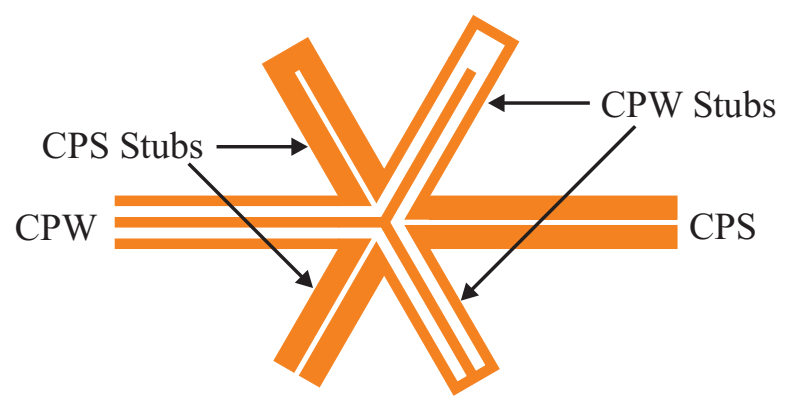

Figure 18: Double-y balun implemented with CPW and CPS lines.

Equations for the characteristic impedance of CPW and CPS lines, derived using quasi-static analysis, are given in [23] and [24]. The characteristic impedance of the $\mathrm{CPW}$ with finite ground planes over a finite substrate is given by

$$
Z_{\text {ocpw }}=\frac{30 \pi}{\sqrt{\varepsilon_{r e}^{c p w}}} \frac{K^{\prime}\left(k_{1}\right)}{K\left(k_{1}\right)}
$$

with

$$
\begin{gathered}
\varepsilon_{r e}^{c p w}=1+\frac{\varepsilon_{r}-1}{2} \frac{K\left(k_{2}\right)}{K^{\prime}\left(k_{2}\right)} \frac{K^{\prime}\left(k_{1}\right)}{K\left(k_{1}\right)} \\
k_{1}=\frac{a}{b} \sqrt{\frac{1-b^{2} / c^{2}}{1-a^{2} / c^{2}}}
\end{gathered}
$$




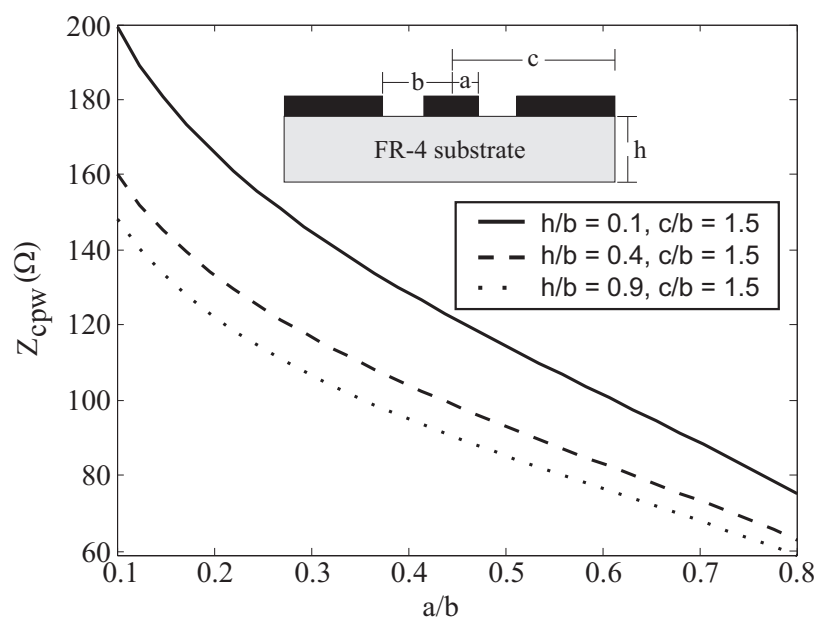

Figure 19: Effect of substrate thickness on CPW characteristic impedance.

$$
k_{2}=\frac{\sinh (\pi a / 2 h)}{\sinh (\pi b / 2 h)} \sqrt{\frac{1-\sinh ^{2}(\pi b / 2 h) / \sinh ^{2}(\pi c / 2 h)}{1-\sinh ^{2}(\pi a / 2 h) / \sinh ^{2}(\pi c / 2 h)}},
$$

where $K$ and $K^{\prime}$ are the complete elliptic integrals of the first kind and its complement, respectively. Figure 19 illustrates impedance curves generated for $\mathrm{CPW}$ lines over FR4 substrate with c/b held constant while varying substrate thickness. It is seen that decreasing substrate thickness increases the characteristic impedance of the CPW line. Figure 20 illustrates impedance curves generated with $\mathrm{h} / \mathrm{b}$ held constant while varying the width of the outer conductor. It is seen from the curves that decreasing the width of the outer conductor, for a given a/b ratio, increases the characteristic impedance of the CPW line. Curves from both figures also illustrate that the characteristic impedance of the $\mathrm{CPW}$ line increases with increasing gap width or decreasing conductor width. Figure 21 illustrates impedance curves for CPW lines over FR4, RT/6006, and Alumina; the characteristic impedance of the CPW lines decreases with increasing substrate permittivity. The smallest achievable gap and conductor widths are limited by manufacturing tolerances.

The characteristic impedance of a CPS line is given by

$$
Z_{\text {ocps }}=\frac{120 \pi}{\sqrt{\varepsilon_{r e}^{c p s}}} \frac{K\left(k_{3}\right)}{K^{\prime}\left(k_{3}\right)}
$$




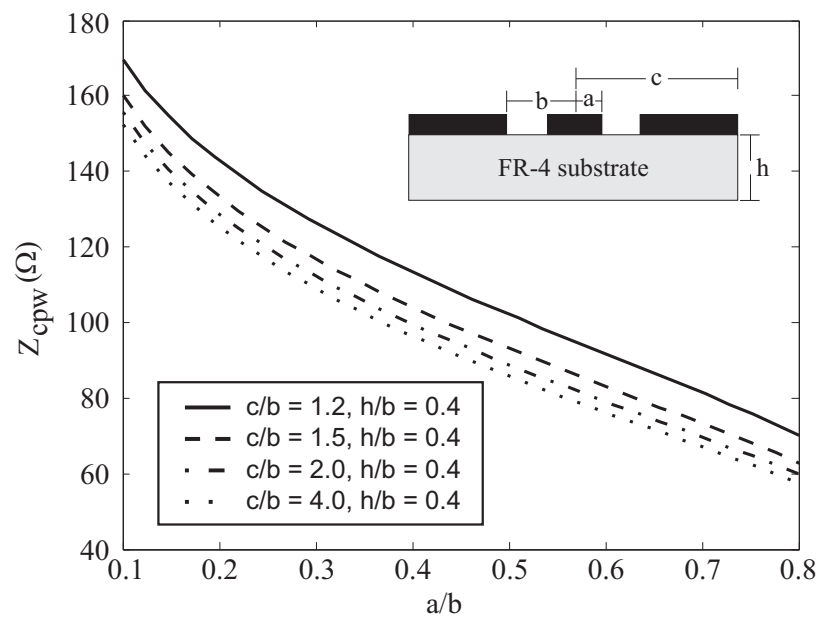

Figure 20: Effect of ground conductor width on CPW characteristic impedance.

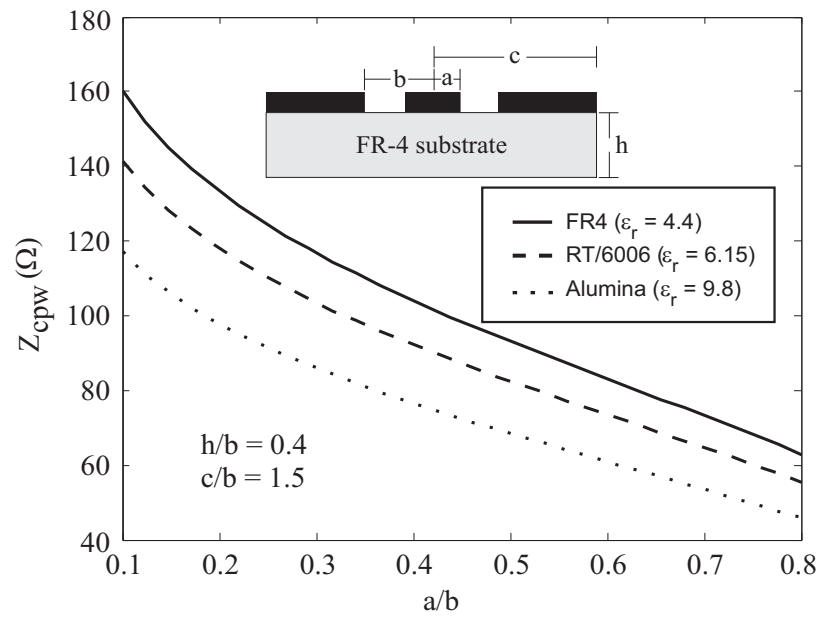

Figure 21: Effect of substrate permittivity on CPW characteristic impedance. 


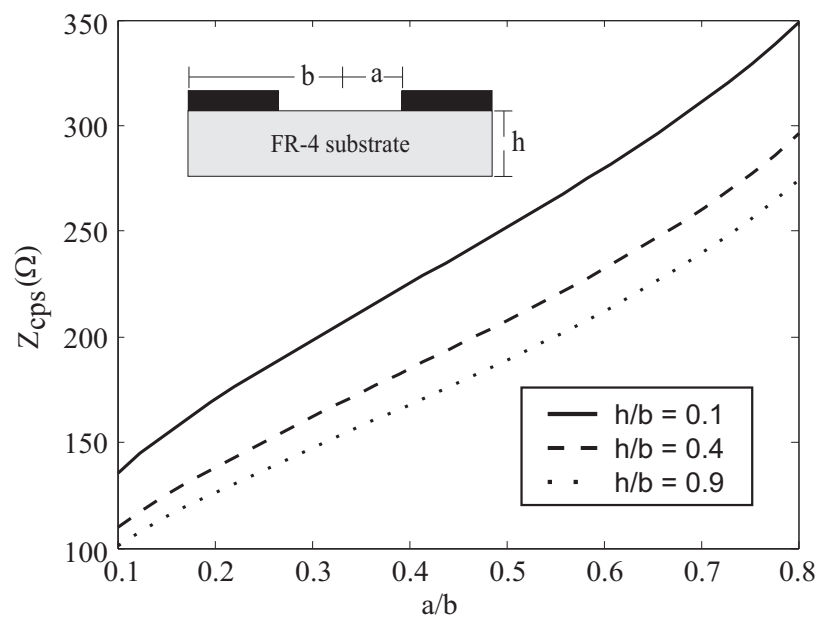

Figure 22: Effect of substrate thickness on CPS characteristic impedance.

with

$$
\begin{gathered}
\varepsilon_{r e}^{c p s}=1+\frac{\varepsilon_{r}-1}{2} \frac{K\left(k_{4}\right)}{K^{\prime}\left(k_{4}\right)} \frac{K^{\prime}\left(k_{3}\right)}{K\left(k_{3}\right)} \\
k_{3}=\frac{a}{b}, \\
k_{4}=\frac{\sinh (\pi a / 2 h)}{\sinh (\pi b / 2 h)},
\end{gathered}
$$

where $a$ and $b$ are defined in Fig. 17b. Figure 22 illustrates CPS impedance curves for various substrate thicknesses. It is seen from the curves that the characteristic impedance of the CPS line decreases with increasing substrate thickness. The curves also illustrate that the characteristic impedance of CPS lines increases with increasing gap width or decreasing conductor width. Figure 23 illustrates impedance curves for CPS lines over FR4, RT/6006, and Alumina; as in the case with CPW lines, the characteristic impedance of CPS lines decreases with increasing substrate permittivity.

Next, the attenuation characteristics in CPW and CPS lines, over FR4 substrate, are studied. Coplanar lines exhibit three types of losses: dielectric, conductor, and radiation/surface wave [23]. Radiation loss due to surface waves can be avoided if a thin substrate is used, that is, the substrate thickness $h$ should be such that

$$
h \sqrt{\varepsilon_{r}}<0.12 \lambda_{o},
$$




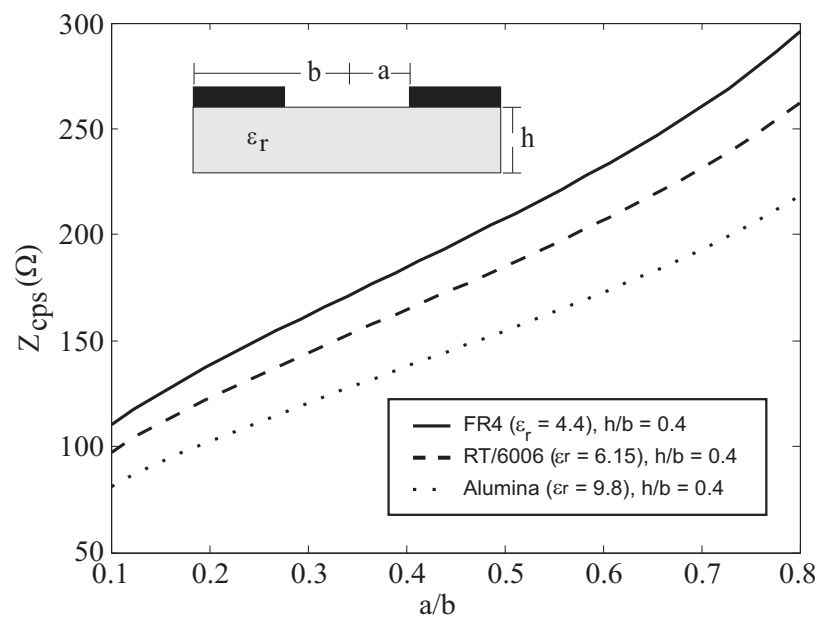

Figure 23: Effect of substrate permittivity on CPS characteristic impedance.

where $\varepsilon_{r}$ is the relative permittivity of the substrate, and $\lambda_{o}$ is the wavelength at the highest frequency [23]. It will be shown in Section 2.4 that the substrate thickness should be minimized for improved performance of the double-y balun. Hence, in this analysis, only dielectric and conductor losses are considered; a thin substrate meeting the requirement in (13) is assumed. The dielectric loss for CPW and CPS lines, in $\mathrm{dB} /$ unit length, is given by

$$
\alpha_{d}=2.73 \frac{\varepsilon_{r}}{\sqrt{\varepsilon_{r e}}} \frac{\varepsilon_{r e}-1}{\varepsilon_{r}-1} \frac{\tan \delta}{\lambda_{o}},
$$

where $\tan \delta$ is the loss tangent of the FR4 substrate $\left(\tan \delta=0.012\right.$ for FR4), $\lambda_{o}$ is the wavelength, and $\varepsilon_{r}$ and $\varepsilon_{r e}$ are the relative and effective permittivities of the $\mathrm{CPW}$ and CPS lines, respectively [23]. In [25], conductor loss for CPW and CPS lines is given, in $\mathrm{dB} /$ unit length, by

$$
\begin{aligned}
\alpha_{c} & =8.68 \frac{R_{s} \sqrt{\varepsilon_{r e}}}{480 \pi K\left(k_{s}\right) K\left(k_{s}^{\prime}\right)\left(1-k_{s}^{2}\right)} \times \\
& \left\{\frac{1}{a}\left[\pi+\log \left(\frac{8 \pi a\left(1-k_{s}\right)}{t\left(1+k_{s}\right)}\right)\right]+\frac{1}{b}\left[\pi+\log \left(\frac{8 \pi b\left(1-k_{s}\right)}{t\left(1+k_{s}\right)}\right)\right]\right\}
\end{aligned}
$$

, where $R_{s}$ is the surface resistivity $\left(8.24 \times 10^{-3} \sqrt{f_{G H z}}\right.$ for copper $), t$ is the conductor thickness (chosen to be 0.7 mils for half-ounce copper), $a$ and $b$ are the dimensions 
of the CPW and CPS lines as defined in Fig. 17, and

$$
\begin{gathered}
k_{s}=\frac{a}{b} \\
k_{s}^{\prime}=\sqrt{1-k_{s}^{2}} .
\end{gathered}
$$

Figure 24 illustrates the total attenuation (defined here as $\alpha_{d}+\alpha_{c}$ ) for CPW lines, computed using Equations 14 and 15, for various characteristic impedances. The center conductor width of the CPW was varied, and the gap and outer conductor widths were synthesized to achieve 75,90 , and $105 \Omega$ characteristic impedances. Figure 25 illustrates the synthesized gap and outer conductor widths for the impedances in Fig. 24. Figure 24 provides us with some insight into the attenuation characteristics of CPW lines. It is seen that the total attenuation increases with frequency; both, conductor and dielectric loss increase with frequency. While Fig. 24 provides us with a range of values for total attenuation in CPW lines, it is seen that the attenuation varies with the physical dimensions of the CPW line [26].

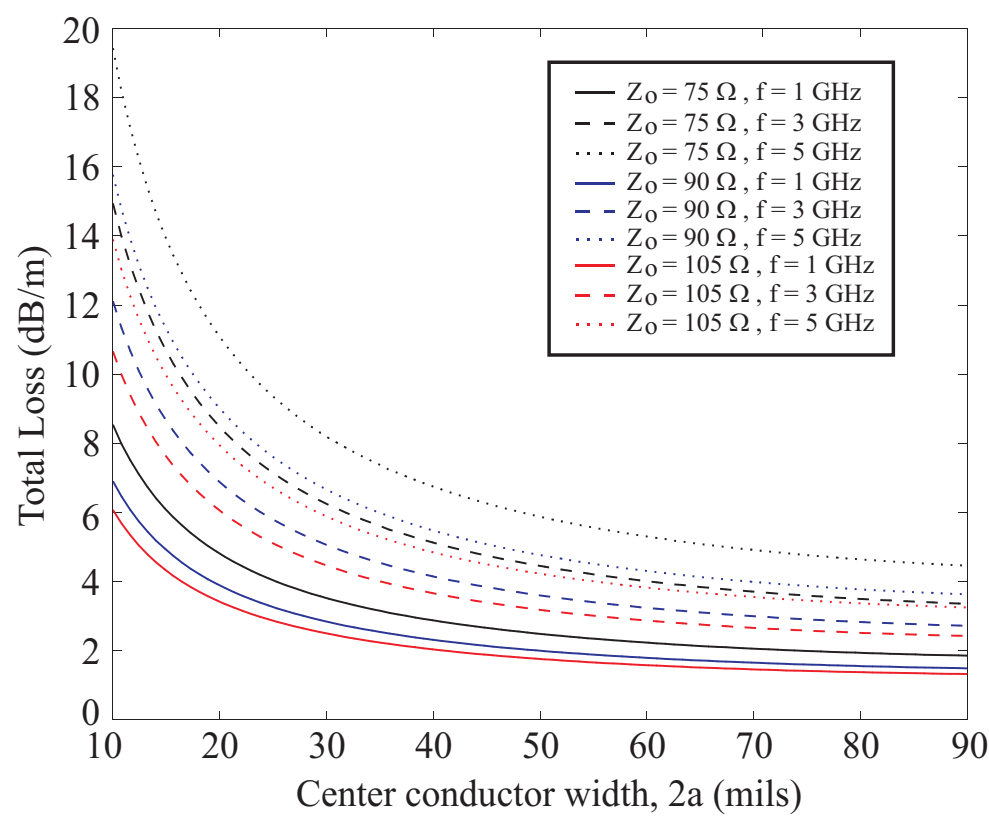

Figure 24: Total attenuation $\left(\alpha_{d}+\alpha_{c}\right)$ computed using Equations 14 and 15 for CPW line in Fig. 17a. 


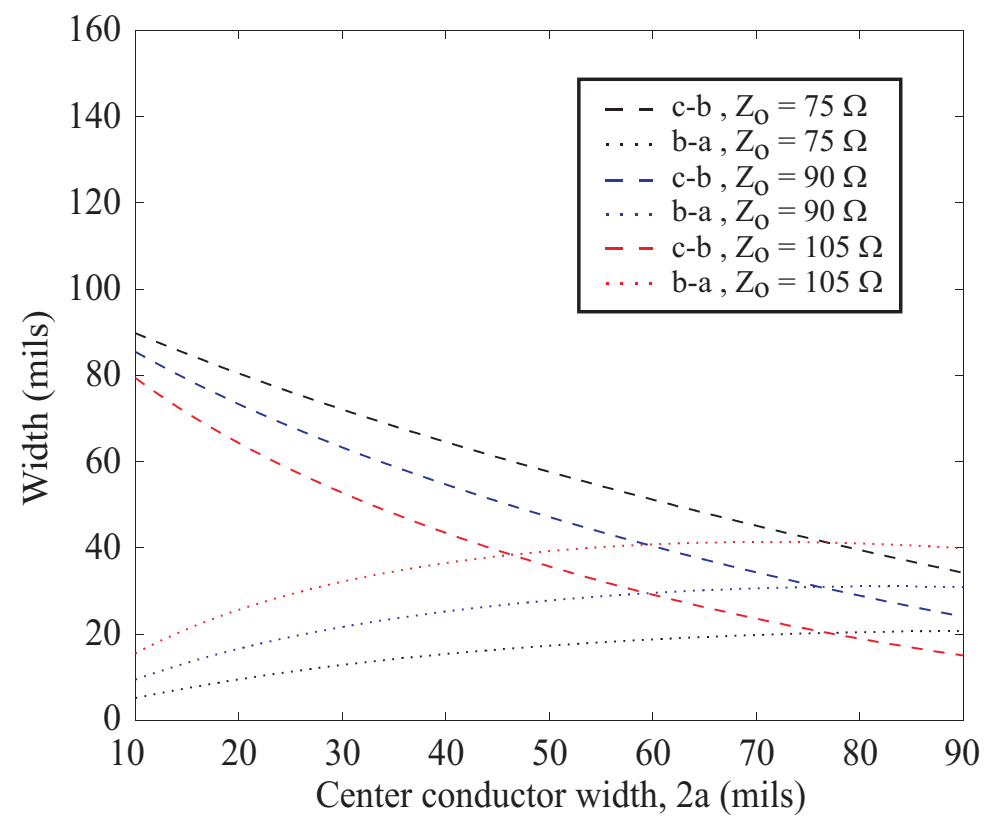

Figure 25: Synthesized gap and outer conductor widths for CPW line in Fig. 17a, with characteristic impedances and center conductor widths illustrated in Fig. 24.

The total attenuation for various synthesized CPS lines is illustrated in Fig. 26. Figure 27 illustrates the synthesized gap and conductor widths for the CPS characteristic impedances in Fig. 26. Equations 14 and 15 were used to compute the total attenuation; Equations 14 and 15 are valid for both, CPW and CPS lines, with appropriate meanings for $a, b$, and $\varepsilon_{r e}$. As was the case for the CPW lines, the attenuation varies with the CPS line's physical dimensions. The total attenuation for CPS lines also increases with increasing frequency.

The double-y balun requires that the CPW and CPS stubs have equal characteristic impedances as well as equal electrical lengths in order to exhibit an all-pass network behavior. In this research, the feasibility of using the double-y balun for feeding pulsed antennas, specifically the resistively loaded V-dipole, is investigated. The resistively loaded V-dipole is a symmetric antenna requiring a balanced feed. Its input impedance is around $200 \Omega$. In order to transition from an unbalanced $50 \Omega$ coaxial line to a symmetric antenna, a balun is required. However, the double-y balun cannot transform impedances inherently, therefore, matching networks are required. 


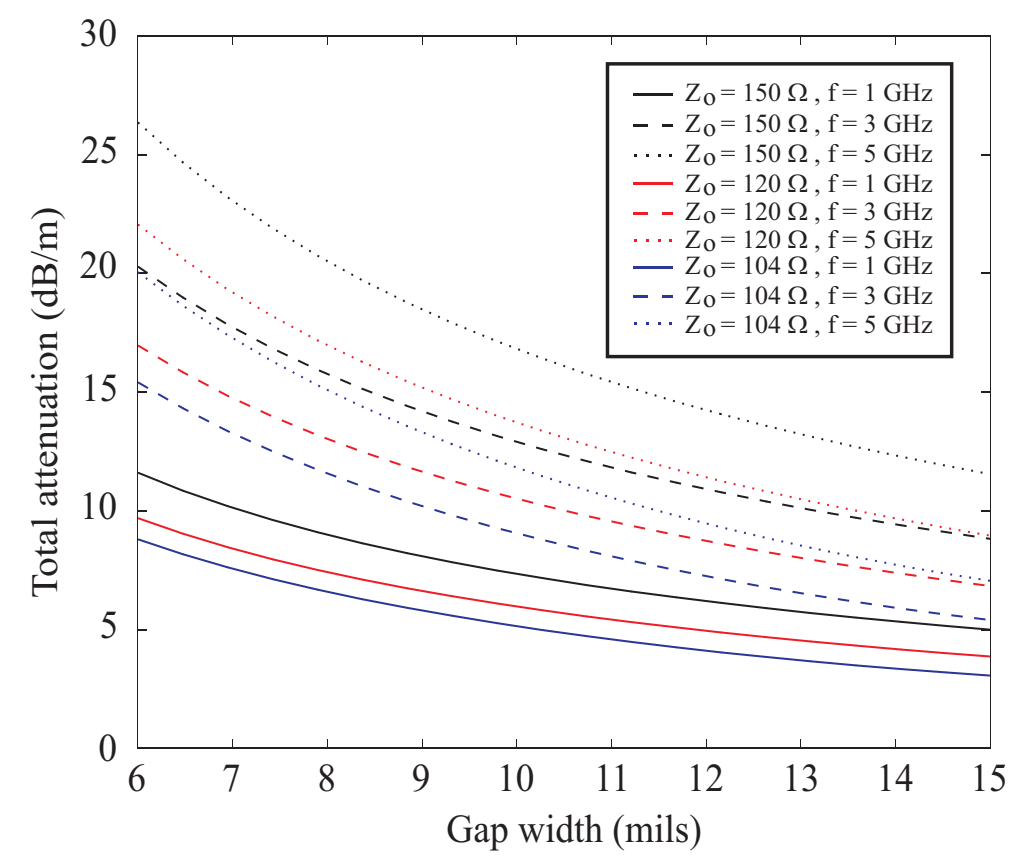

Figure 26: Total attenuation $\left(\alpha_{d}+\alpha_{c}\right)$ computed using Equations 14 and 15 for CPS line in Fig. 17b.

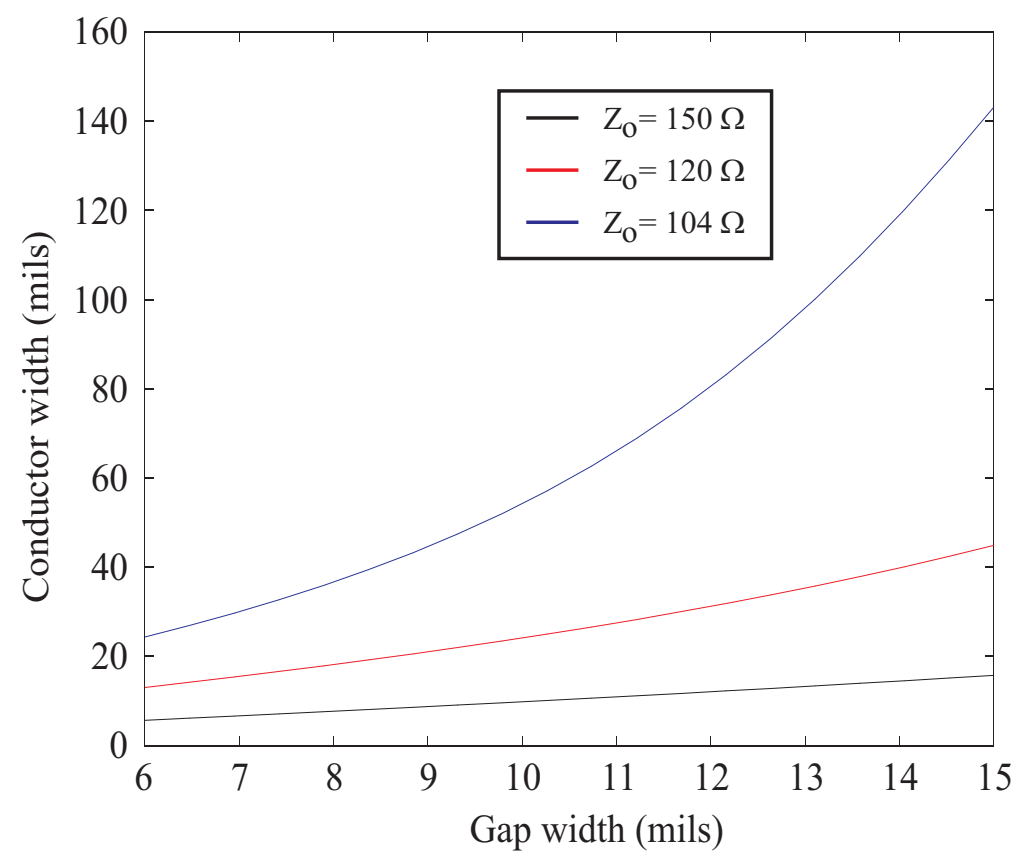

Figure 27: Synthesized gap and conductor widths for CPS line in Fig. 17b, with characteristic impedances and gap widths illustrated in Fig. 26.

From Figs. 19 and 22, it is seen that it is difficult to achieve $50 \Omega$ CPS lines and $200 \Omega \mathrm{CPW}$ lines. However, there exists a range of impedance values that can be 
realized with both CPW and CPS lines (impedance of the CPW and CPS sections of the balun must be equal). The design approach taken in this research, as illustrated in Fig. 28, is to design impedance tapers to transition from the $50 \Omega$ unbalanced coaxial feedline to the balun and to transition from the balun to the $200 \Omega$ antenna.

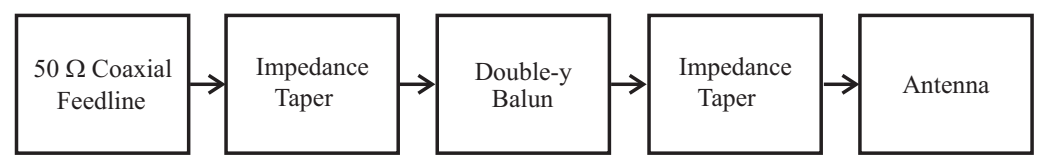

Figure 28: Design approach to transition from coaxial feedline to double-y balun and resistively loaded V-dipole.

\subsection{Momentum vs. High Frequency Structure Sim- ulator (HFSS)}

The double-y balun of interest in this research (implemented with coplanar waveguide (CPW) and coplanar strip (CPS) lines) was analyzed numerically using Hewlett Packard's Momentum and Ansoft's High Frequency Structure Simulator (HFSS), both commercial electromagnetic simulators. Momentum is a method of moments code while HFSS is a finite-element code. Three computers were put together for the purpose of running the simulations locally; two PCs each with a $1.8 \mathrm{GHz}$ Athlon processor and 3 Gigs RAM and a third PC with a $2 \mathrm{GHz}$ Athlon processor and 1.5 Gigs RAM. All three PCs were running the Windows XP operating system. A program was written in Matlab with the aid of Microwave Artwork Toolbox to draw double-y baluns in gerber format. The resulting gerber file was translated to dxf format and imported into Momentum and HFSS.

Analyzing the double-y balun with two different electromagnetic simulators provides a means by which the numerical results can be validated with better certainty. Figure 29 illustrates a double-y balun designed to transition from an unbalanced $104 \Omega \mathrm{CPW}$ section to a balanced $104 \Omega$ CPS section over 58 mil thick FR4 substrate $\left(\varepsilon_{r}=4.4, \tan \delta=0.012\right)$. The $\mathrm{CPW}$ and CPS lines were synthesized so 
that $\mathrm{w}_{c c p w}+2 \mathrm{w}_{g c p w}+2 \mathrm{~g}_{c p w}=2 \mathrm{w}_{c p s}+\mathrm{g}_{c p s}$, subject to the following constraints: minimum $\mathrm{w}_{c c p w}=10$ mils (based on design in [18]), minimum $\mathrm{g}_{c p s}=6$ mils (based on manufacturing tolerances). The resulting impedance at which this occurred was computed to be $104 \Omega$, as illustrated in Fig. 30. CPW bridges placed near the junction are necessary for proper operation of the balun. The function of the CPW bridges will be addressed later. Figure 31 illustrates this double-y balun modeled and meshed in Momentum. Ports (P1-P5 in Fig. 31) were placed along the CPW and CPS sections; ports enable energy to flow in and out of the circuit. Impedance tapers were omitted to reduce computational time significantly.

To excite the proper mode along the CPW section, P1 was defined to be an internal port; P2 and P3 were defined to be ground reference ports for P1. To excite the proper mode along the CPS section, P4 and P5 were defined to be differential ports (ports with opposite polarity). With these port configurations, Momentum treats P1, P2, and P3 to be one port and P4 and P5 to be a second port. Therefore, simulation of this structure with the above port configuration yields two-port s-parameters.

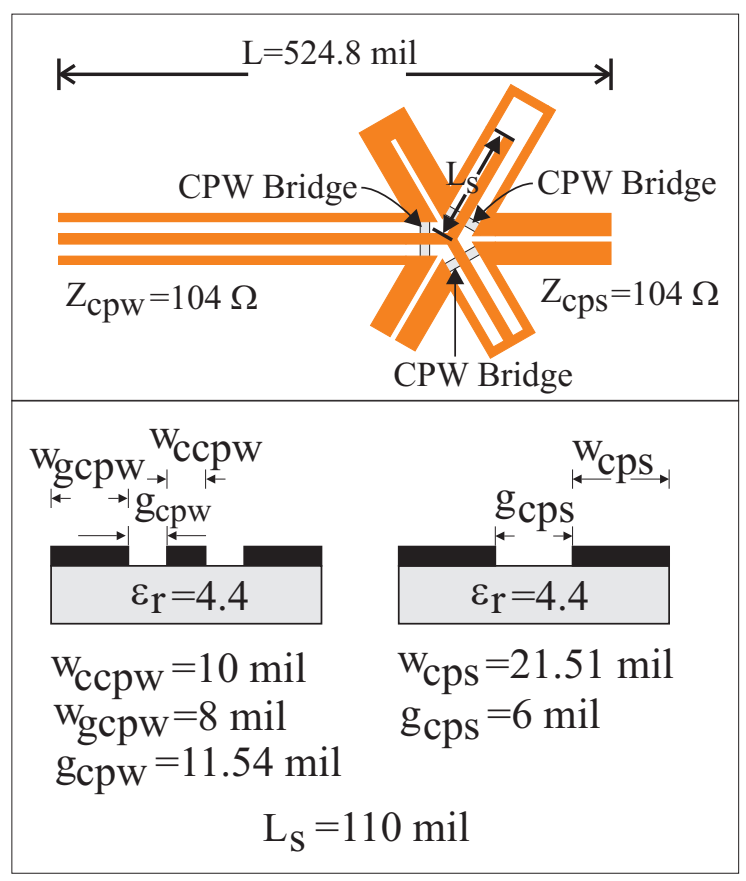

Figure 29: Dimensions of double-y balun modeled in Momentum and HFSS. 


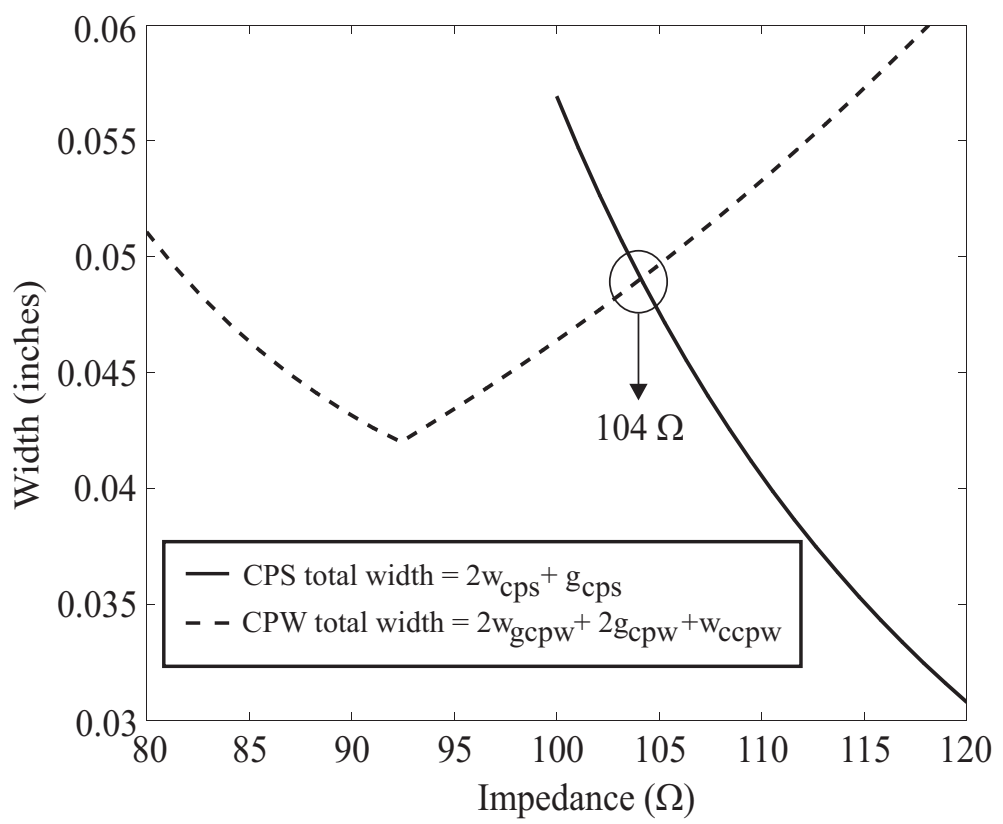

Figure 30: Plot illustrating impedance vs. minimum achievable total width of CPW and CPS lines over 58 mil FR4 substrate subject to following constraints: minimum $\mathrm{w}_{c c p w}=10$ mils, minimum $\mathrm{g}_{c p s}=6$ mils.

Internal ports do not remove mismatches at the port boundary for s-parameter calculations. Since P1 was defined to be an internal port, the reference impedance of the port defaults to $50 \Omega$. Therefore, if the characteristic impedance of the CPW section is different from $50 \Omega$, as is the case for the $104 \Omega$ CPW section in Fig. 29, the resulting s-parameters must be re-normalized to reference the appropriate characteristic impedance. Conversion of s-parameters based on a given set of reference impedances to a new s-matrix based on a new set of reference impedances is discussed in Appendix A.

In Momentum, the substrate was modeled as extending infinitely along the plane containing the double-y balun. The substrate characteristics were modeled as illustrated in Fig. 32. The CPW and CPS lines were drawn on the cond layer and mapped as strip. Mapping a layer as strip causes Momentum to assign conductive properties to the objects drawn on the layer (in this case objects on cond layer are treated as PEC). In contrast, mapping a layer to slot causes all objects drawn on the layer to be 
non-conductive and the surrounding layer to be conductive. The vias (diameter $=6$ mil) were drawn on the hole layer, which was mapped as via. Mapping a layer to via causes objects drawn on the layer to be conductive and cut vertically through one or more substrate layers. The jumpers (width=10 mil) connecting the vias were drawn on the cond2 layer and mapped as strip along the bottom of the substrate as illustrated in Fig. 32b. Dielectric loss was modeled in the substrate by adding a loss tangent value of 0.012 for the FR4 substrate. The simulation frequency plan was setup linearly, from $1 \mathrm{GHz}$ to $13 \mathrm{GHz}$ with increments of $0.1 \mathrm{GHz}$.

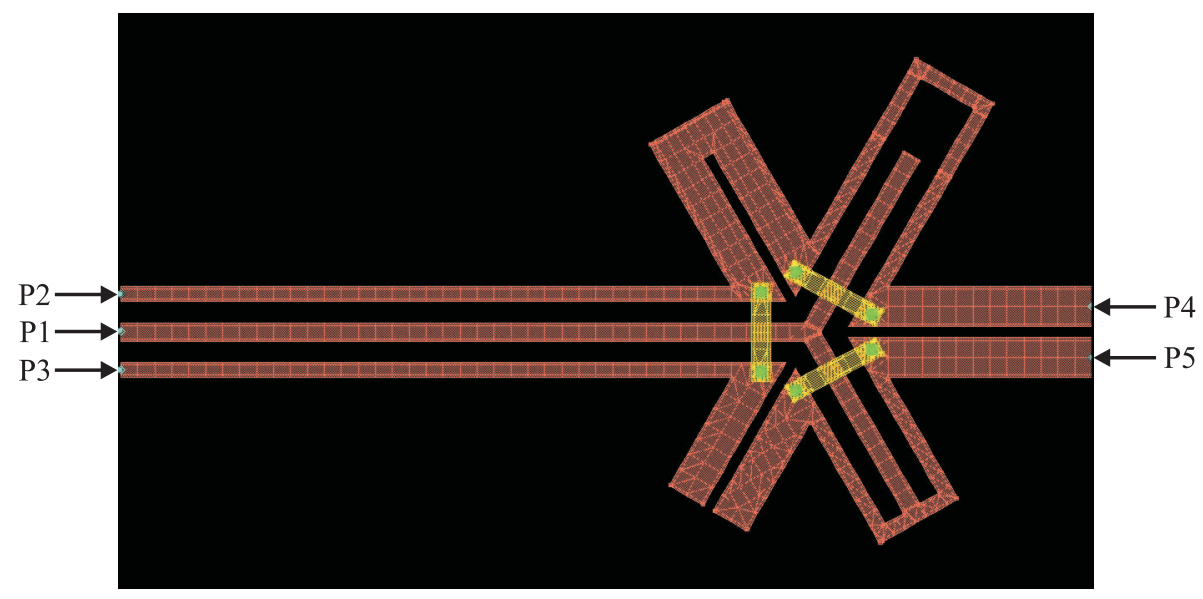

Figure 31: Double-y balun modeled and meshed in Momentum.

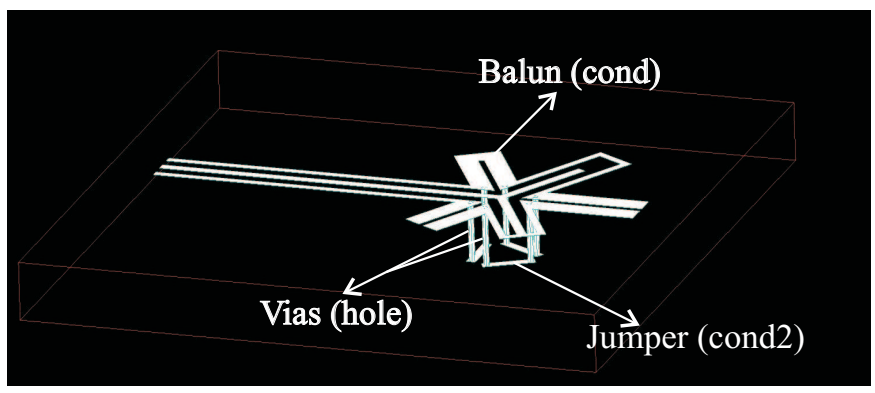

(a)
FreeSpace

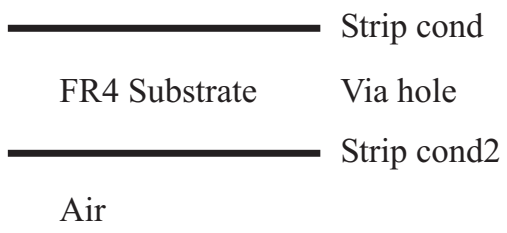

(b)

Figure 32: Illustration of (a) 3-D view of double-y balun and (b) substrate characteristics as defined in Momentum.

Simulation results from Momentum for the double-y balun illustrated in Fig. 29 are illustrated in Figs. 33 and 34. These figures illustrate that increasing the minimum 
Table 1: Momentum simulation summary for balun modeled as illustrated in Fig. 31.

\begin{tabular}{|c|c|c|}
\hline Parameter & 30 cells/wavelength & 60 cells/wavelength \\
\hline Unknown Currents & 1528 & 2715 \\
\hline Number of Frequency Points & 124 & 124 \\
\hline Total Simulation Time & $03 \mathrm{~h} .56 \mathrm{~m} .12 \mathrm{sec}$. & $11 \mathrm{~h} .47 \mathrm{~m} .15 \mathrm{sec}$. \\
\hline
\end{tabular}

mesh density of the balun (balun meshed at $13 \mathrm{GHz}$ ) from 30 to 60 cells/wavelength had little effect on the computed results. The specified mesh density is the minimum mesh density used by Momentum when meshing the balun; the actual mesh could be denser in some regions. Table 1 provides a simulation summary for both cases. Both cases correspond to simulations running on a $1.8 \mathrm{GHz}$ Athlon PC.

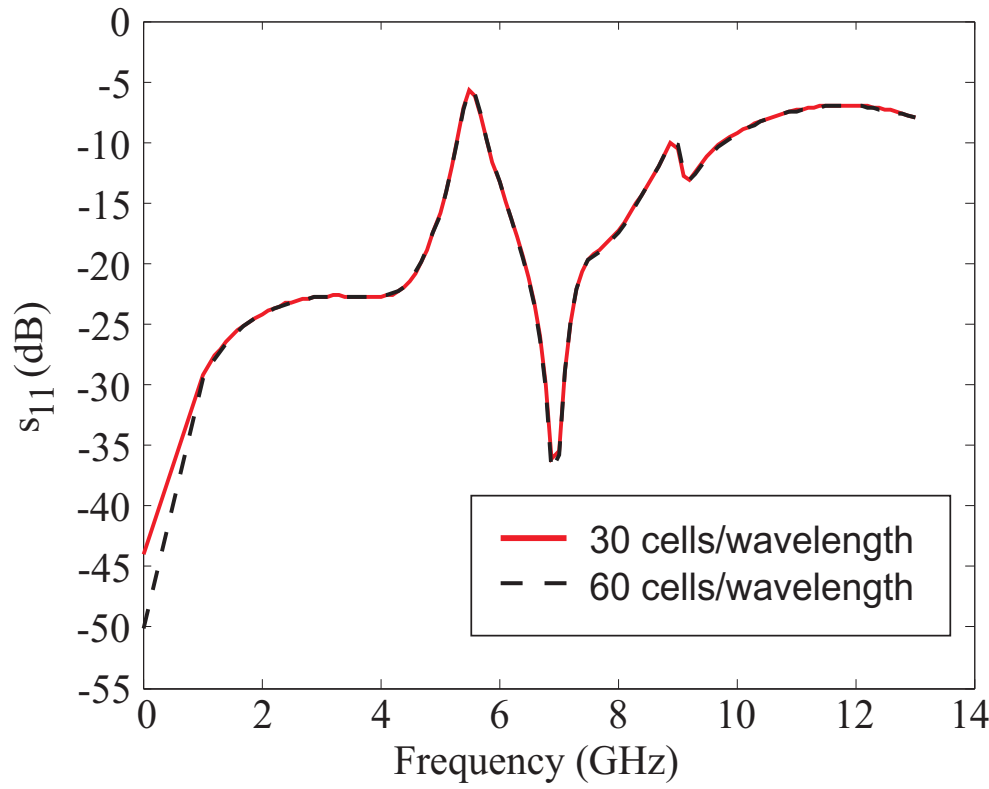

Figure 33: Momentum $s_{11}$ results for two different meshes (mesh generated at 13 $\mathrm{GHz})$.

The balun in Fig. 29 was also modeled in HFSS, as illustrated in Fig. 35. The CPW and CPS sections were excited using lumped ports, as illustrated in Fig. 35. Lumped ports excite a simplified, single-mode field excitation assuming a given reference impedance for s-parameter referencing. The reference impedances were chosen 


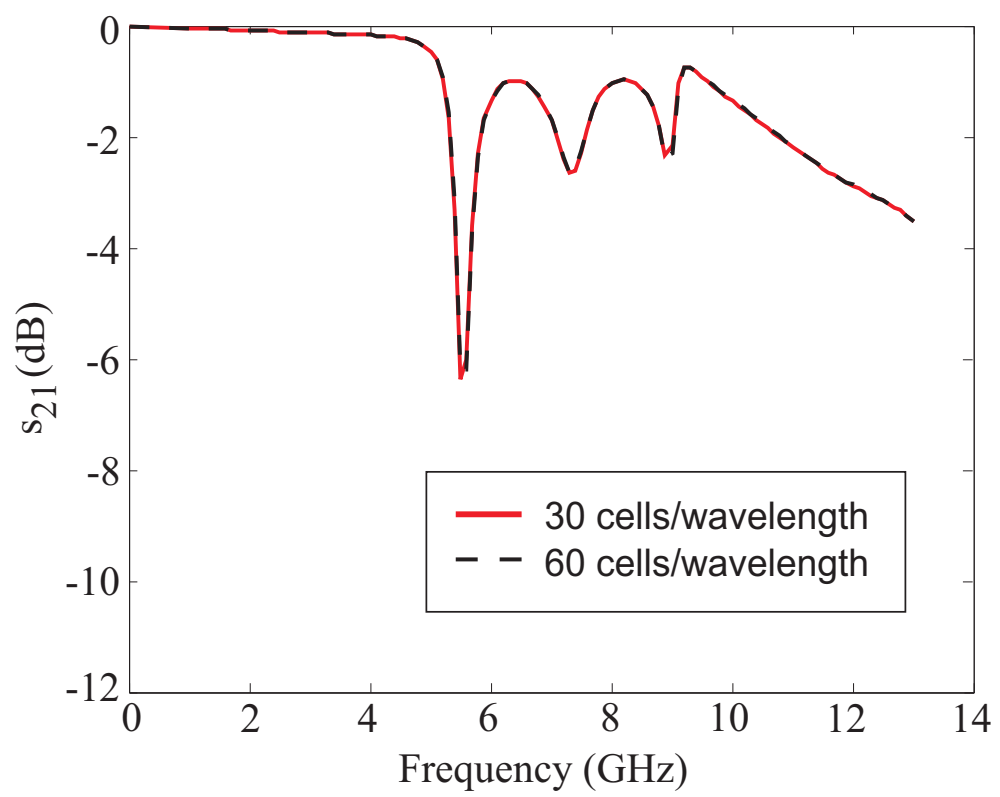

Figure 34: Momentum $s_{21}$ results for two different meshes (mesh generated at 13 $\mathrm{GHz})$.

from impedance equations for CPW and CPS lines in [23]. All conductors were modeled as PEC and the substrate was modeled with a dielectric loss of 0.012. Dimensions of vias and CPW bridges were the same as those in the Momentum model. Convergence criteria for the solution was established by setting a tolerance on the maximum change in the magnitude of the s-parameters between two consecutive passes. During each simulation pass, HFSS calculates the s-parameters and proceeds to refine the mesh if the convergence criteria is not met. A tolerance of 0.01 was chosen as the maximum allowable change in the s-parameters between two passes. This tolerance was chosen to yield reasonable simulation times as well as memory consumption.

HFSS is a 3-D finite-element based code that solves for the field quantities within a bounded region. Therefore, unlike the FR4 substrate modeled in Momentum, the FR4 substrate modeled in HFSS has finite dimensions (in Momentum only the substrate thickness was finite). The balun was enclosed in an air bounded region, as illustrated in Fig. 36, and surrounded with perfectly matched layers (PMLs). The 
outer boundary of the PML was assigned a PEC boundary condition in order to minimize reflections from the interface. The perfectly matched layers act as absorbers that truncate the computational region while physically modeling unbounded space. Although the PML is a very effective absorbing medium, it cannot be placed arbitrarily close to the structure being analyzed (in this case the balun) [27].

Appropriate spacing and thickness of the PML layers was determined by simulating the model illustrated in Fig. 36 with various PML spacings and thicknesses. Using the equations in $[28,29]$, it was determined that 5 PML layers were needed, and the thickness of each PML layer was calculated to be $0.6 \mathrm{~cm}$; equations in [28] provide the number of PML layers as well as the thickness of each layer. However, when automating the PML creation in HFSS, HFSS creates a single PML layer of variable thickness. Instead of having multiple layers along each face of the bounding box, only one layer of PML is created. Therefore, the single PML layer in HFSS was modeled with a thickness of $3 \mathrm{~cm}$ (5 PML layers, each $0.6 \mathrm{~cm}$ thick, were needed). The minimum PML spacing ( $\mathrm{s}_{p m l}$ in Fig. 36) was chosen to be $\lambda_{o} / 30$ at the lowest frequency (simulated from $1 \mathrm{GHz}$ to $13 \mathrm{GHz}$ with spacing of $0.1 \mathrm{GHz}$ ). It is seen from Figs. 37 and 38 that there is negligible deviation in the results when increasing the PML spacing beyond $1 \mathrm{~cm}$ and increasing the PML thickness beyond $3 \mathrm{~cm}$. The simulation time for the case with a PML spacing of $1 \mathrm{~cm}$ and PML thickness of $3 \mathrm{~cm}$ was $13 \mathrm{~h}: 08 \mathrm{~m}: 45 \mathrm{sec}$.

Scattering parameters obtained for the double-y balun in Fig. 29 from Momentum and HFSS are illustrated in Figs. 39 and 40. Resonances limiting the bandwidth of the double-y balun are observed in the results obtained from both, Momentum and HFSS. These resonances are studied more in depth shortly, in Section 2.3. From Figs. 39 and 40, it is seen that the resonant points predicted by Momentum and HFSS agree closely. The discrepancy in the $\mathrm{s}_{11}$ curves could result from the modified CPW feed point illustrated in Fig. 35 as well as numerical error resulting from both 


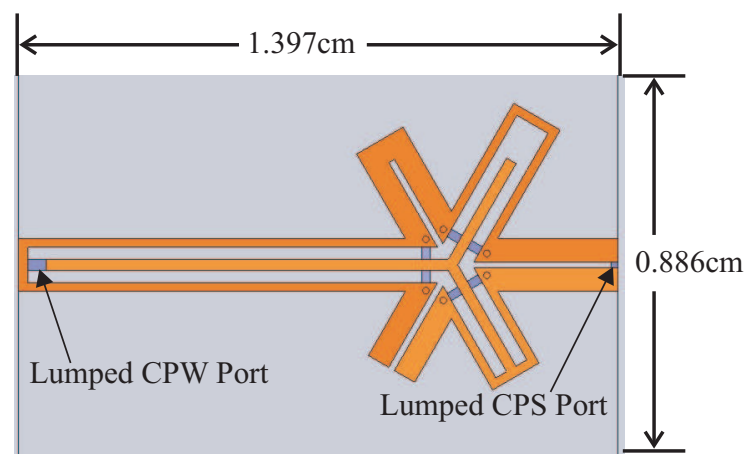

(a)

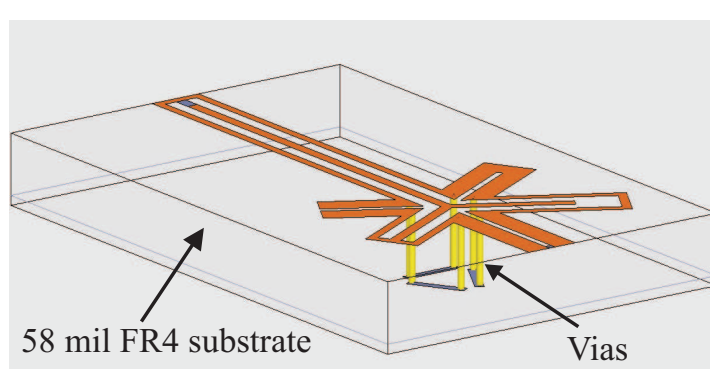

(b)

Figure 35: Illustration of (a) top and (b)3-D view of double-y balun modeled in HFSS.

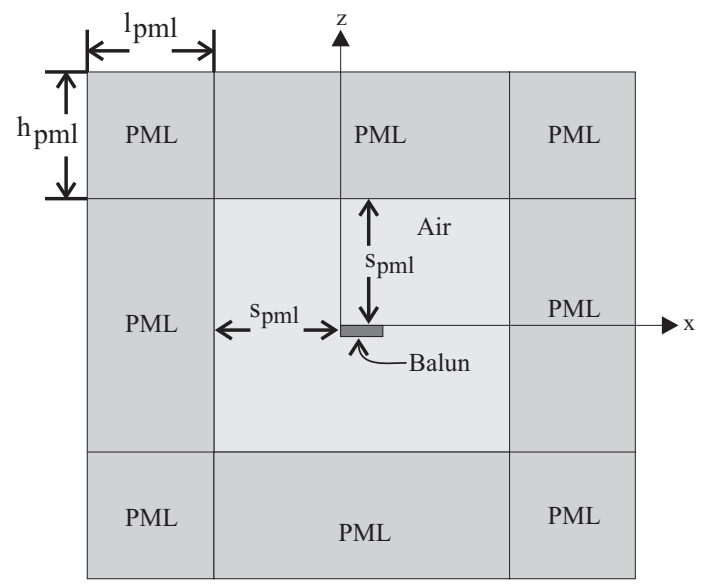

(a)

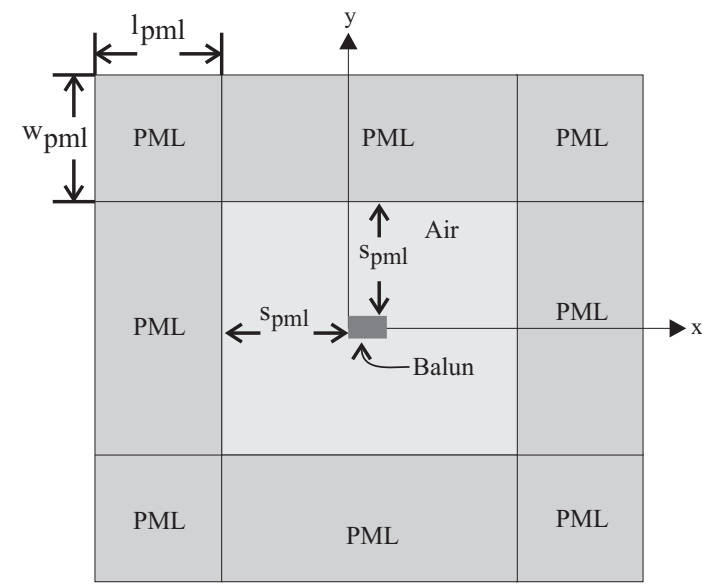

(b)

Figure 36: Illustration of (a) cross-section and (b) top view of double-y balun modeled in HFSS within bounded region. 


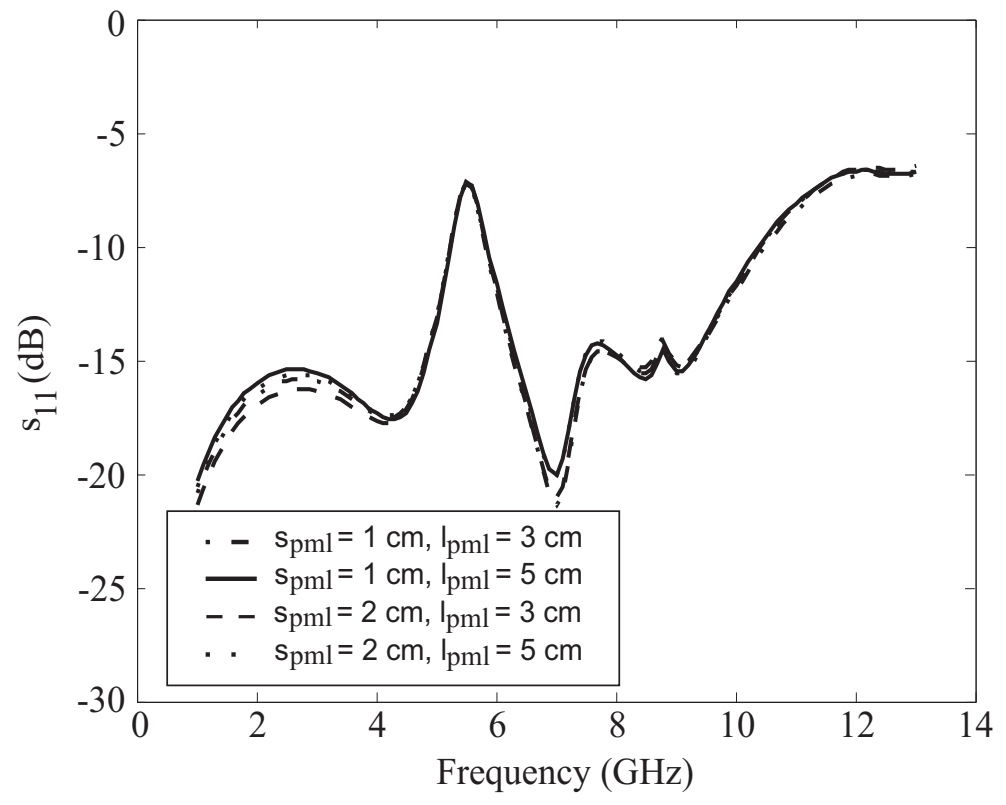

Figure 37: HFSS s $s_{11}$ results for double-y balun in Fig. 29 with various PML configurations.

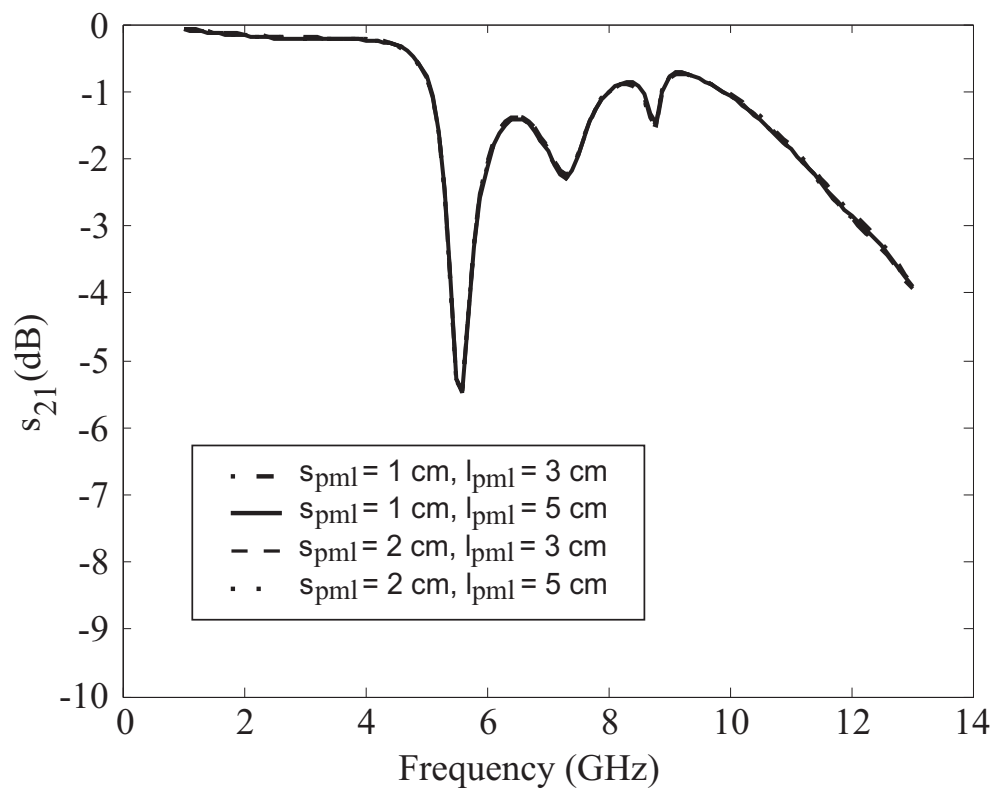

Figure 38: HFSS $s_{21}$ results for double-y balun in Fig. 29 with various PML configurations. 


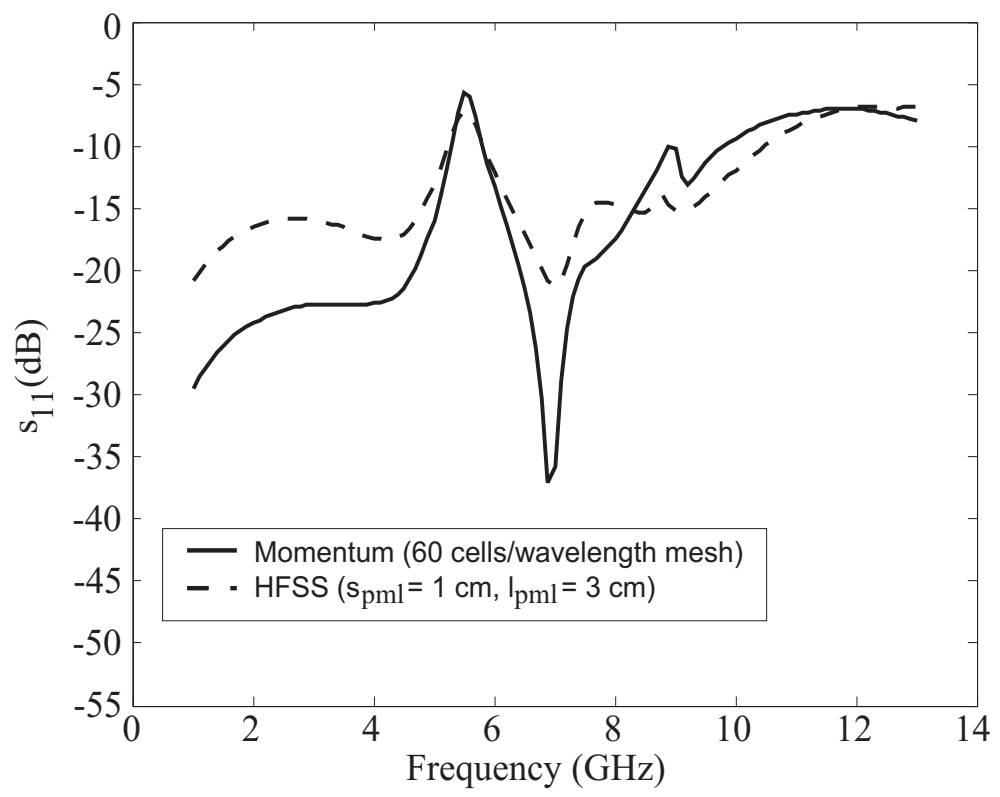

Figure 39: Momentum vs. HFSS $s_{11}$ results for double-y balun in Fig. 29.

codes. However, the resonant points obtained with both simulators agree very well, and the $s_{21}$ results agree very closely. Thus, obtaining converging s-parameter data from two different simulators helps determine whether resonances that appear (may not be predicted theoretically) are due to the double-y balun or numerical error from the different codes (e.g. reflections from the PML, improper port excitations, etc.). Having obtained converging results from both commercial codes, these simulators can be used with better confidence to further study design aspects of the double-y balun. 


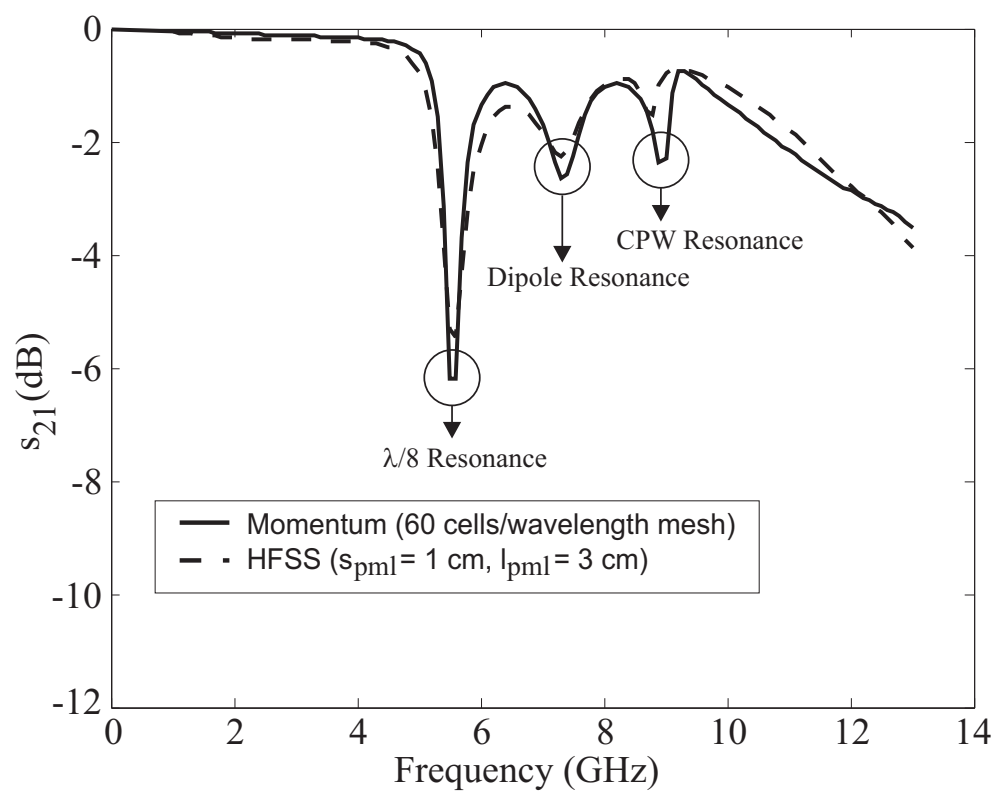

Figure 40: Momentum vs. HFSS $s_{21}$ results for double-y balun in Fig. 29. 


\subsection{Resonant Modes}

The bandwidth of the double-y balun is limited by resonances observed in the $\mathrm{s}_{21}$ plots in Section 2.2. In this section, these resonances are studied, and their effects on the performance of the double-y balun designed in this research, is investigated.

\subsection{1 $\lambda / 8$ Resonance}

The bandwidth of the double-y balun has been reported in $[17,20,16,22]$ to be limited by the lengths of the open and shorted coplanar waveguide (CPW) and coplanar strip (CPS) stubs. Due to junction parasitics and unequal dispersion between the CPW and CPS lines, the balun's performance deteriorates as the lengths of the stubs $\left(\mathrm{L}_{s}\right.$ in Fig. 29) approach $\lambda / 8$. Figure 41 illustrates a double-y balun implemented with CPW and CPS lines, along with an equivalent lumped element model. The CPW bridges at the balun junction are necessary for the equivalent model in Fig. 41b to be valid. Hence, these bridges are necessary for proper operation of the double-y balun. To understand the cause of the $\lambda / 8$ resonance, the input impedance of the double-y balun looking into the unbalanced section was determined by deriving the two port z-matrix. The z-matrix components were derived to be

$$
\begin{aligned}
& z_{11}=\frac{\left(z_{6}+z_{5}\right)\left(z_{2}+z_{3}\right)}{z_{2}+z_{3}+z_{5}+z_{6}} \\
& z_{14}=\frac{z_{2}\left(z_{3}+z_{5}\right)-z_{3}\left(z_{6}+z_{2}\right)}{z_{2}+z_{3}+z_{5}+z_{6}} \\
& z_{41}=\frac{z_{5}\left(z_{2}+z_{3}\right)-z_{3}\left(z_{5}+z_{6}\right)}{z_{2}+z_{3}+z_{5}+z_{6}} \\
& z_{44}=\frac{\left(z_{6}+z_{2}\right)\left(z_{5}+z_{3}\right)}{z_{2}+z_{3}+z_{5}+z_{6}}
\end{aligned}
$$


where

$$
\begin{aligned}
z_{2} & =j Z_{c p s} \tan \left(\beta_{c p s} l_{c p s}\right) \\
z_{3} & =-j Z_{c p w} \cot \left(\beta_{c p w} l_{c p w}\right) \\
z_{5} & =j Z_{c p w} \tan \left(\beta_{c p w} l_{c p w}\right) \\
z_{6} & =-j Z_{c p s} \cot \left(\beta_{c p s} l_{c p s}\right)
\end{aligned}
$$

are the input impedances looking into the CPW and CPS stubs, and $Z_{c p w}$ and $Z_{c p s}$ are the characteristic impedances of the CPW and CPS sections, respectively. The input impedance looking into the unbalanced section of the balun is then given by

$$
Z_{i n}=z_{11}-\frac{z_{14} z_{41}}{\left(z_{44}+Z_{o}\right)}
$$

when port 4 is terminated with an impedance $Z_{o}$. If $Z_{c p w}=Z_{c p s}=Z_{o}, \beta_{c p w}=\beta_{c p s}$, and $l_{c p w}=l_{c p s}$, then the input impedance looking into the CPW section of the balun, given by Equation 26, is matched for all frequencies. However, because of unequal dispersion between the CPW and CPS lines, limitations on manufacturing tolerances that affect $Z_{c p w}$ and $Z_{c p s}$, and effective lengths of the stubs due to fringing, a mismatch results in the input impedance, $Z_{\text {in }}$, as the lengths of the stubs approach $\lambda / 8$. This is illustrated in Fig. 42, where the input impedance looking into the CPW section is plotted for various characteristic impedances of the CPW stubs (with all stubs having equal electrical lengths). The characteristic impedance of the CPS stubs and the load are normalized to $1 \Omega$. When the characteristic impedances of the CPW and CPS stubs are equal to each other and equal to the load impedance, the input impedance is matched at all frequencies. However, if the characteristic impedance of the CPW stubs is varied, a mismatch occurs as the electrical length approaches $45^{\circ}$ (or $\lambda / 8$ ).

To further validate the theoretical equations derived above, the lumped model in Fig. 41b was modeled in HP Advanced Design System (ADS), as illustrated in Fig. 43. ADS is a high frequency circuit simulator. Figure 44 illustrates a plot of $\mathrm{s}_{21}$ 


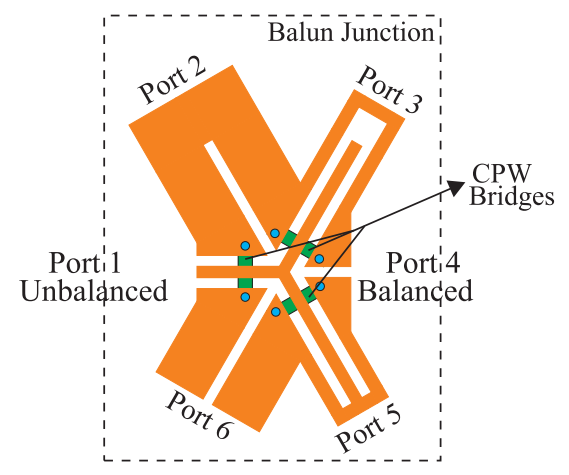

(a)

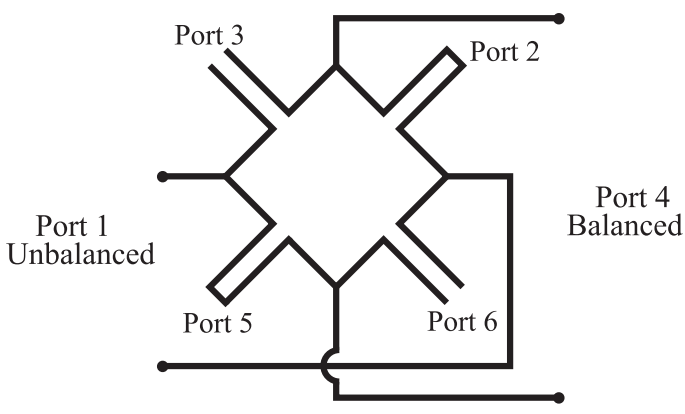

(b)

Figure 41: Illustration of (a) double-y balun implemented with CPW and CPS lines and (b) equivalent lumped element model.

comparing theoretical results obtained from Equations 18-21 (z-matrix converted to s-matrix), with results obtained via the ADS model. Both cases model the double-y balun with 110 mil CPW and CPS stubs over FR4 substrate. For the mismatched case, the characteristic impedance of the $\mathrm{CPW}$ lines is severely mismatched with the impedance of the CPS lines (impedance of the CPW lines is twice the impedance of the CPS lines). The $\lambda / 8$ resonance is observed with both models when $Z_{c p w} \neq Z_{c p s}$, and the resonance occurs when the stub lengths are $\lambda / 8$.

To obtain a better understanding of the $\lambda / 8$ resonance, we examine the equivalent circuit of the double-y balun, illustrated in Fig. 15b in Chapter 1. It is seen that without a load across Port 4, the equivalent circuit is a resonant circuit. However, by adding a load across Port 4, maintaining equal characteristic impedances of the CPW and CPS lines (equal to each other and to the load impedance), and maintaining equal electrical lengths of the CPW and CPS stubs, the resonant behavior of the circuit is removed. However, if one of the above conditions is perturbed, then the circuit becomes prone to resonating. When the junction does resonate, the input impedance is no longer matched over all frequencies, as illustrated in Fig. 42. From Fig. 42, it is seen that the junction resonance occurs when the stub lengths are $\lambda / 8$ (assuming equal electrical lengths of the CPW and CPS stubs). This was further validated via the ADS results in Fig. 44. Hence, this resonance is referred to as the $\lambda / 8$ resonance. 
For the double-y balun in Fig. 29 (with 110 mil stubs), the $\lambda / 8$ resonance should occur near $8 \mathrm{GHz}$. However, numerical results obtained from Momentum and HFSS, illustrated in Fig. 40, show the $\lambda / 8$ resonance occurring near $6 \mathrm{GHz}$. To investigate this further, the double-y balun in Fig. 29 was modeled in Momentum with smaller CPW and CPS input/output sections (the lengths of the input and output CPW and CPS lines were designed to be 110 mils) to remove all other possible resonances from the passband. Figure 45 illustrates the results from Momentum for the doubley balun with 80 mil and 110 mil stubs. As expected, the $\lambda / 8$ resonance occurs at a higher frequency for the balun with shorter stubs. However, it is seen that both resonances occur at frequencies lower than predicted. Furthermore, the $\lambda / 8$ resonance is more pronounced in Figs. 40 and 45 than that predicted by Fig. 42 (characteristic impedances of the CPW and CPS lines are very close). It is clear that a discrepancy exists between theoretical results obtained from the equivalent circuit model in Fig. $41 \mathrm{~b}$ and numerical results obtained using Momentum and HFSS. The cause of this discrepancy is studied in Section 2.4. 


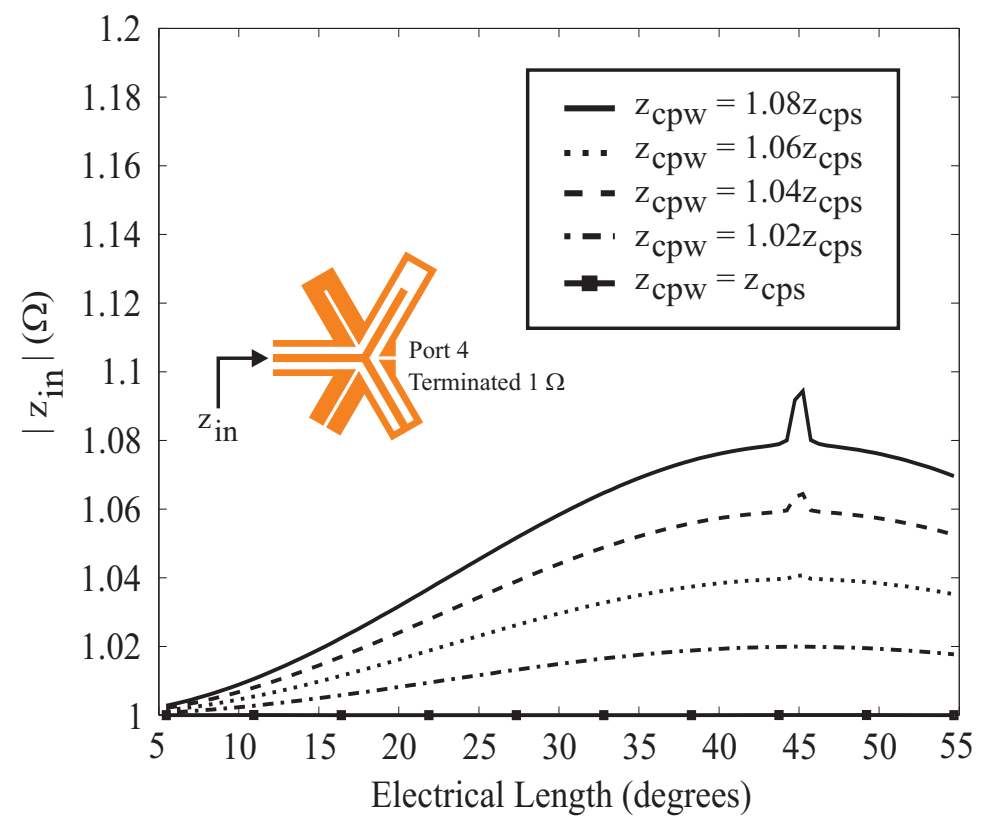

Figure 42: Magnitude of input impedance looking into CPW section of balun junction. Balun is matched at all frequencies when characteristic impedances of CPW and CPS stubs are equal to the terminated load (normalized to $1 \Omega$ ).

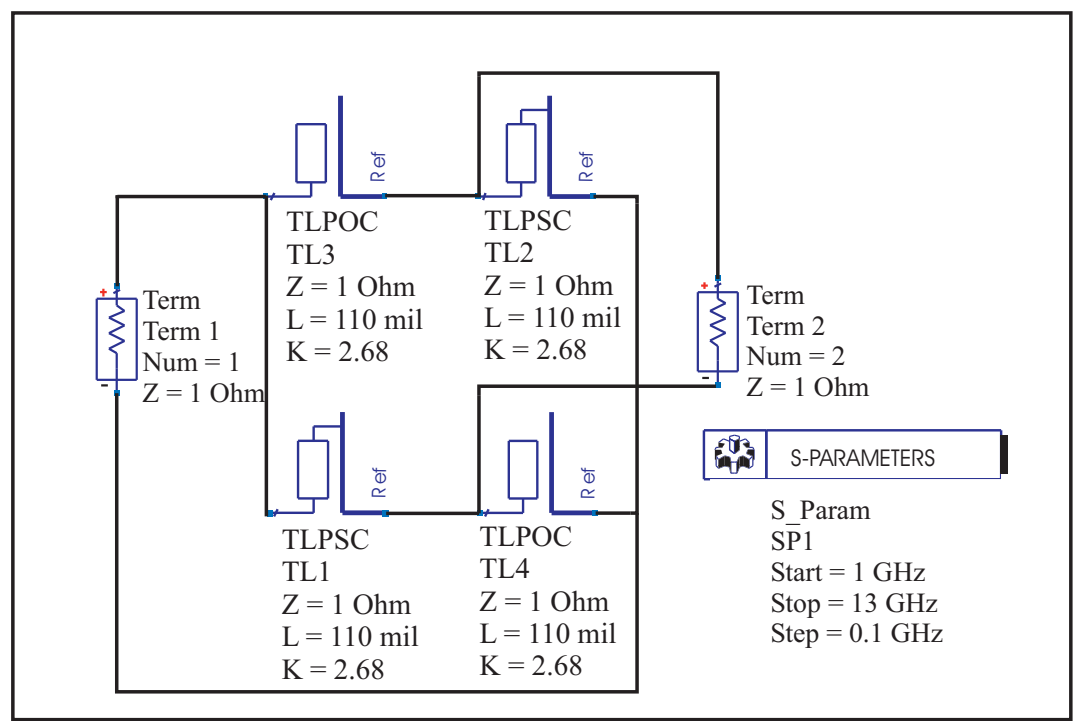

Figure 43: ADS implementation of the double-y balun lumped element model illustrated in Fig. 41. 


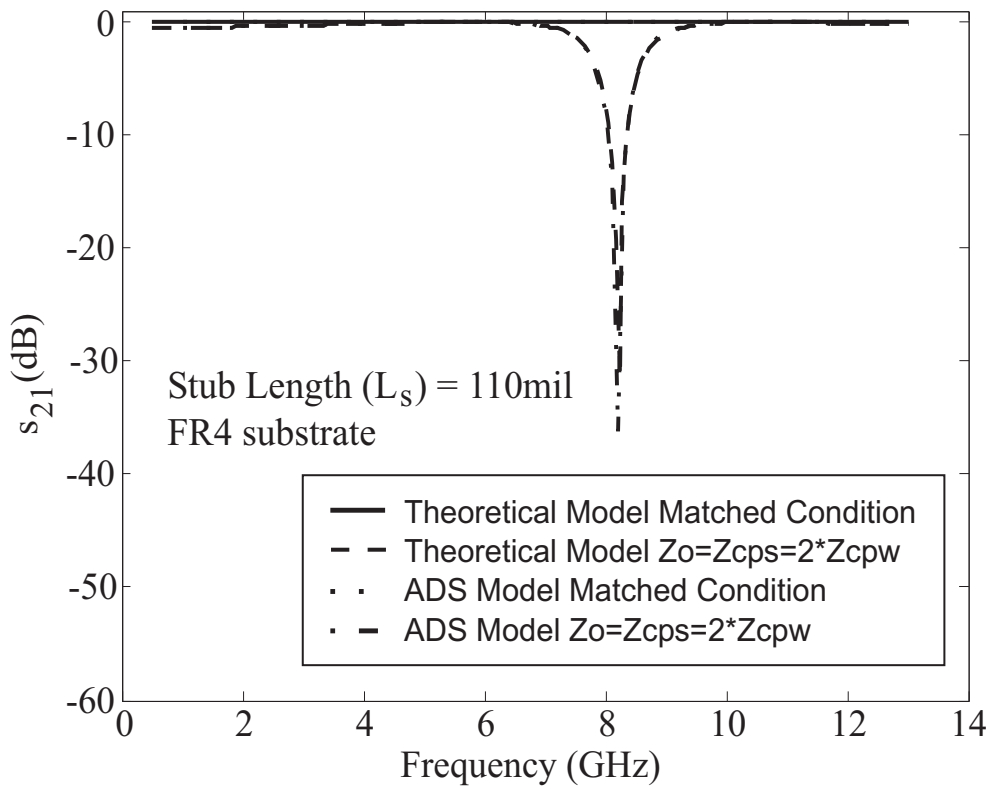

Figure 44: Plot of $\mathrm{s}_{21}$ comparing theoretical results from Equation 18 with ADS model. Theoretical and ADS curves overlap for the matched case. Theoretical and ADS curves for the mismatched case also overlap.

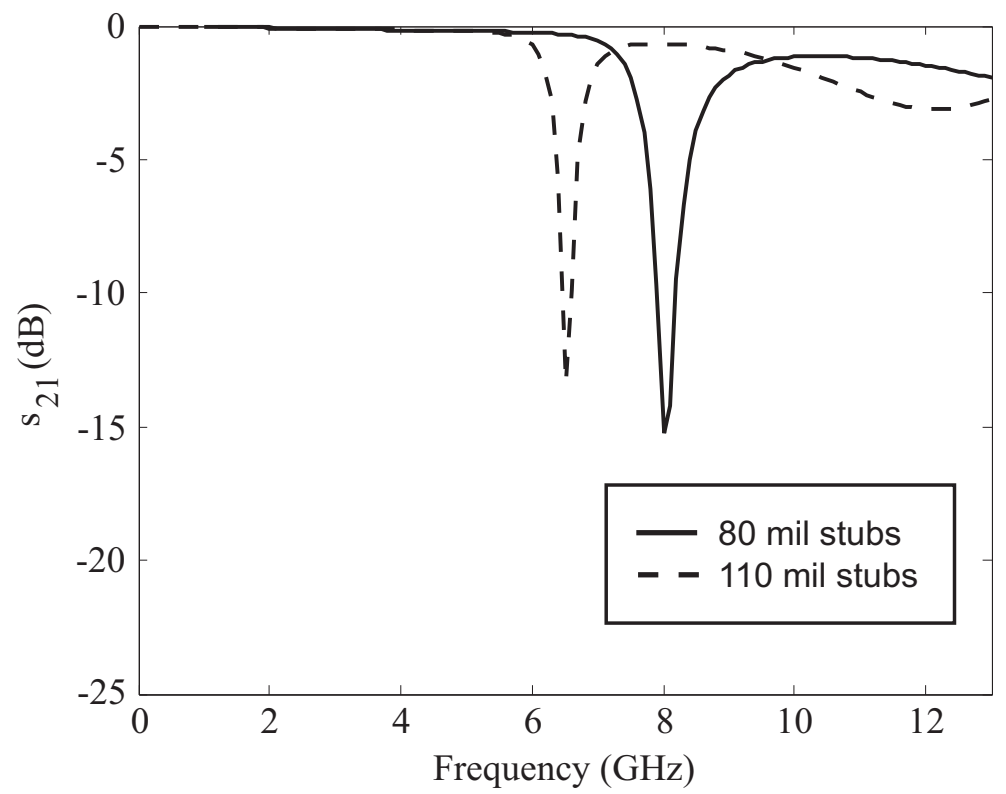

Figure 45: Momentum $\mathrm{s}_{21}$ results for double-y balun with 80 mil and 110 mil stubs. 


\subsubsection{CPW Resonance}

A resonant mode along the ground conductors of the CPW input line has been reported in [20]. This mode is illustrated in Fig. 46 and is caused by differential-mode currents along the outer ground conductors. The outer ground conductors form a shorted transmission line, as illustrated in Fig. 46b, which resonates for lengths equal to multiples of a half-wavelength. Since the resonant frequency depends on the length of the input CPW section, this resonance can be pushed out of the balun's passband by minimizing the length of the CPW line. However, since the double-y balun designed in this research requires an impedance taper to transition from a $50 \Omega$ coaxial line to the balun, it is difficult to minimize the input CPW section and move the resonance out of the passband.

Another approach towards removing the CPW resonance in the balun's passband is to add an additional CPW bridge along the input section, as illustrated in Fig. 47 [20]. The use of CPW bridges to eliminate the resonance along the outer CPW ground conductors is investigated in this section. Specifically, this section deals with two questions that arise when designing the additional CPW bridges: (1) how close to the balun junction should the additional CPW bridge be placed and (2) how many additional CPW bridges should be placed along the CPW section.

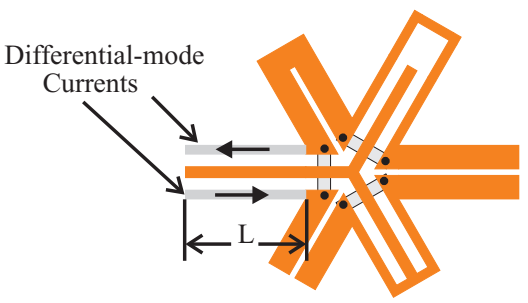

(a)

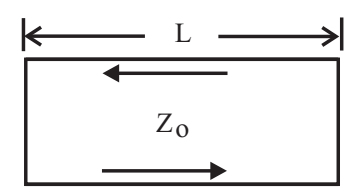

(b)

Figure 46: Illustration of (a) differential-mode currents along outer CPW ground conductors and (b) equivalent transmission line circuit.

In Section 2.2, the balun in Fig. 29 was analyzed numerically using Momentum and HFSS. Figure 48 illustrates the $\mathrm{s}_{21}$ plot for this balun, obtained via Momentum, 


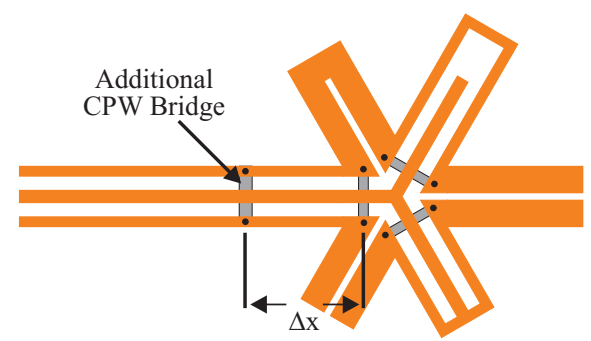

Figure 47: Illustration of double-y balun with additional CPW bridge to remove CPW resonance along outer ground conductors.

with the $\lambda / 8$ and CPW resonances labeled. In addition, Figure 48 illustrates $\mathrm{s}_{21}$ results for the double-y balun in Fig. 29 with and without an additional CPW bridge. It is seen from Fig. 48 that the additional CPW bridge cannot be placed arbitrarily close to the CPW bridge at the balun junction. Placing the additional CPW bridge 140 mils from CPW bridge at the balun junction yielded better results than placing the additional bridge 20 mils from the junction bridge.

The double-y balun designed in this research requires impedance tapers for both, the CPW and CPS sections. These tapers increase the overall length of the balun considerably, therefore, it is desirable to investigate the CPW resonance (this resonance is dependent on the input CPW line length) for the double-y balun with impedance tapers. However, modeling the balun with the impedance tapers requires significant computational time. Therefore, the balun in Fig. 29 was modeled in Momentum with extended CPW and CPS lines (length of each section was 1.6 inches), as illustrated in Fig. 49. These extended lines require a less complicated mesh, thereby decreasing computational time substantially. Figure 50 illustrates Momentum simulation results for the double-y balun in Fig. 49 with and without additional CPW bridges. It is seen that an additional CPW bridge is required to suppress the CPW resonances near 2.2 $\mathrm{GHz}$ and $4.4 \mathrm{GHz}$. Adding further $\mathrm{CPW}$ bridges does seem to offer improved $\mathrm{s}_{21}$ as seen from Fig. 49. 


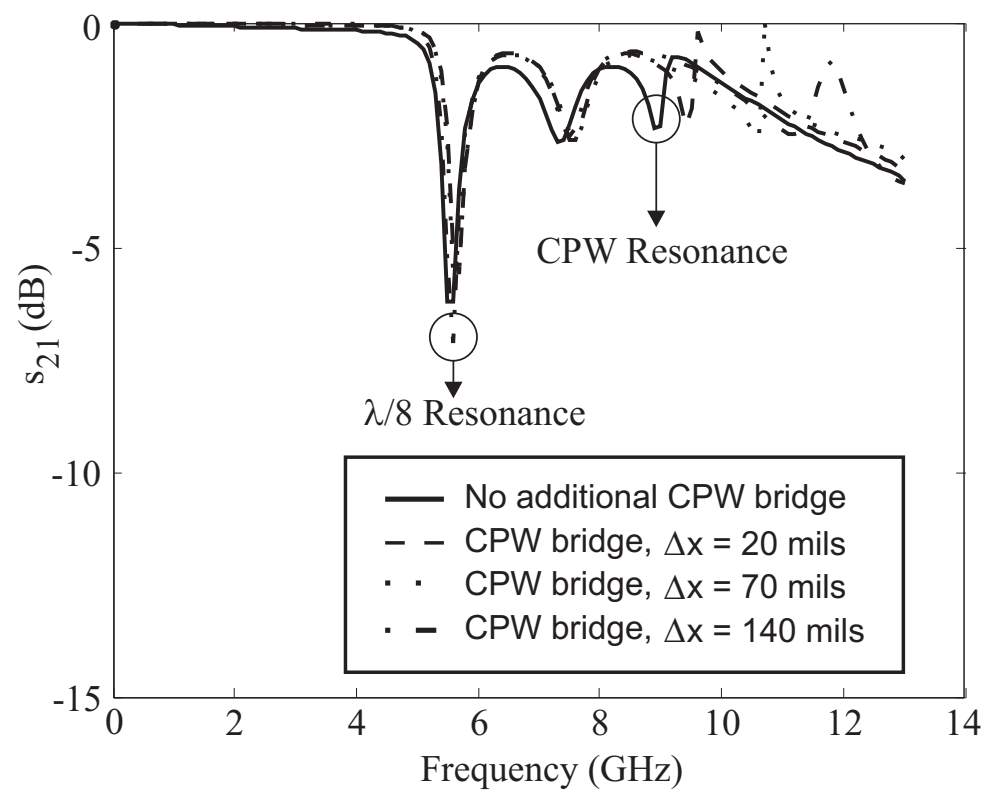

Figure 48: Momentum $\mathrm{s}_{21}$ results for double-y balun with and without additional CPW bridge.

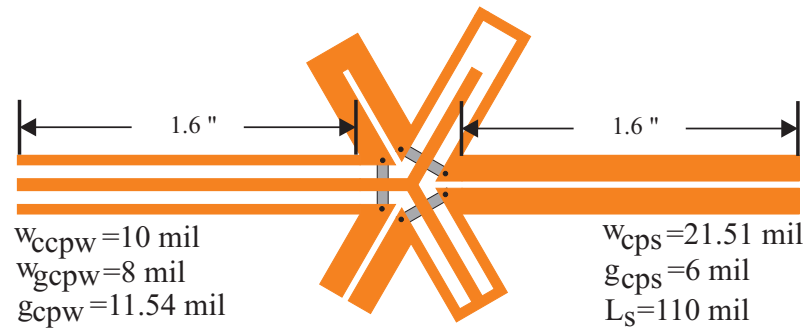

Figure 49: Illustration of double-y balun with extended CPW and CPS sections. 


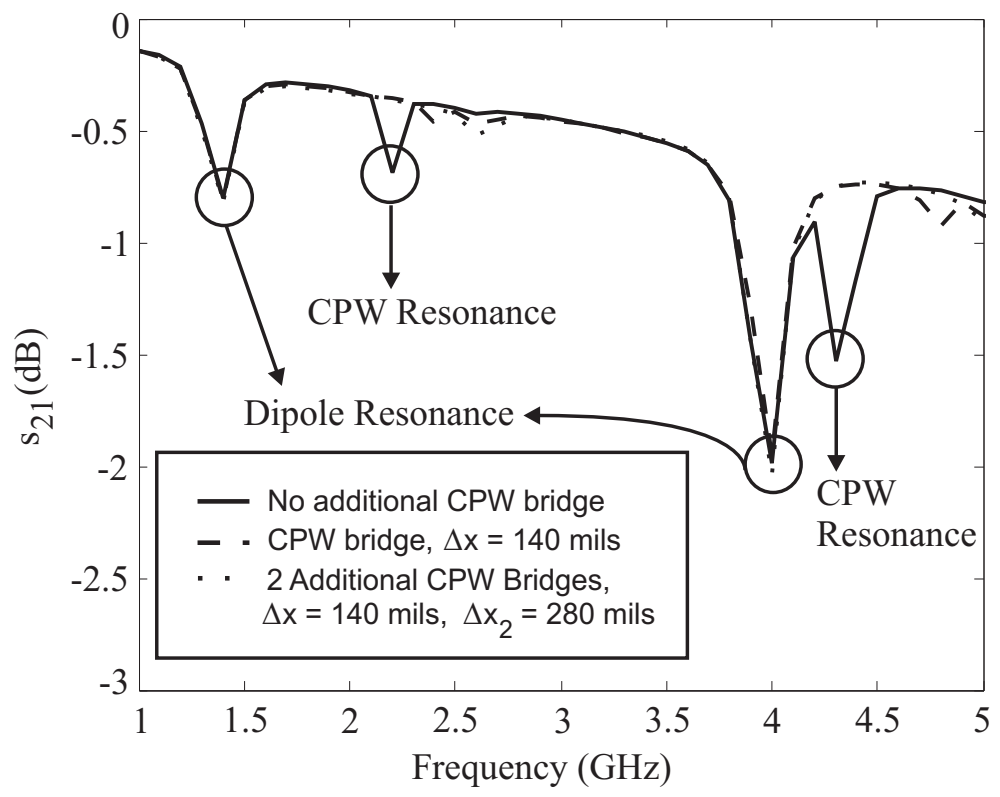

Figure 50: Momentum $\mathrm{s}_{21}$ results for double-y balun in Fig. 49 with and without additional CPW bridges.

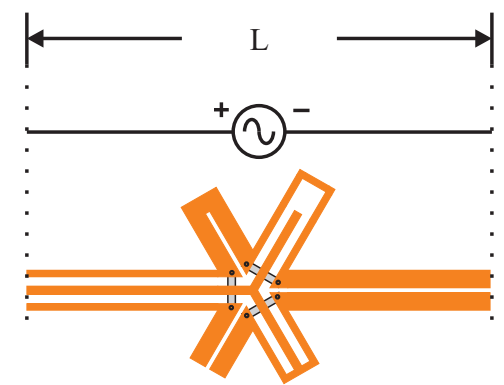

Figure 51: Illustration of 'dipole' resonance excited at balun junction, causing CPW and CPS lines to act as dipole arms.

\subsubsection{Dipole Resonance}

In [20], the double-y balun was enclosed in a conductive enclosure, and a resonant mode was found to occur when the overall length of the balun was equal to odd multiples of a half-wavelength. The resonant mode was identified as being a 'dipole' resonance, as illustrated in Fig. 51: a resonance excited at the balun junction causing the CPW and CPS lines to act as dipole arms. The presence of this mode indicates that the balun is not achieving perfect balancing; the mode conversion from CPW to CPS is not perfect. The 'dipole' resonance, observed in experimental results in 
[20], resulted from enclosing the double-y balun in a conductive enclosure. In this research work, the balun length is truncated when modeled numerically. Therefore, this resonance appears in the numerical simulations. While the balun is truncated for numerical analysis, the balun in this research is not designed to be in a conductive enclosure. Practically, it may be difficult to observe this mode because of the loss introduced by the impedance tapers as well as the coaxial lines connecting the CPW section of the balun to the network analyzer. Therefore, although this resonance is observed in the numerical results, it may not be noticeable in measured results.

\subsection{CPW Bridge Design-Substrate Thickness}

The double-y balun in Fig. 29 was designed with 110 mil stubs over 58 mil FR4 substrate. Results in Figs. 42 and 44 predict the $\lambda / 8$ resonance to occur around $8 \mathrm{GHz}$ for the double-y balun with 110 mil stubs over FR4. Numerical results for the double-y balun in Fig. 29 were illustrated in Section 2.2. It is seen that the resonance due to the $\lambda / 8$ limitation occurs near $6 \mathrm{GHz}$, not $8 \mathrm{GHz}$ as predicted by the lumped element model. In addition, this resonance is more pronounced in the results obtained from the Momentum and HFSS simulations (the deep resonance in Fig. 44 was obtained by severely mismatching the CPW and CPS characteristic impedances). To determine the cause of these discrepancies between results obtained from the full-wave simulation of the double-y balun and the results obtained from simulating the equivalent lumped circuit model, the lumped element model in Fig. $41 \mathrm{~b}$ was modified to include the effects of the CPW bridges.

The CPW bridges are necessary for the operation of the double-y balun. These bridges are to have negligible impedance (ideally should have zero impedance); they are meant to be shorted jumpers across the outer CPW conductors. The actual impedance of these bridges can be determined by calculating the input impedance looking into these bridges (bridges act as a transmission line extending through the 


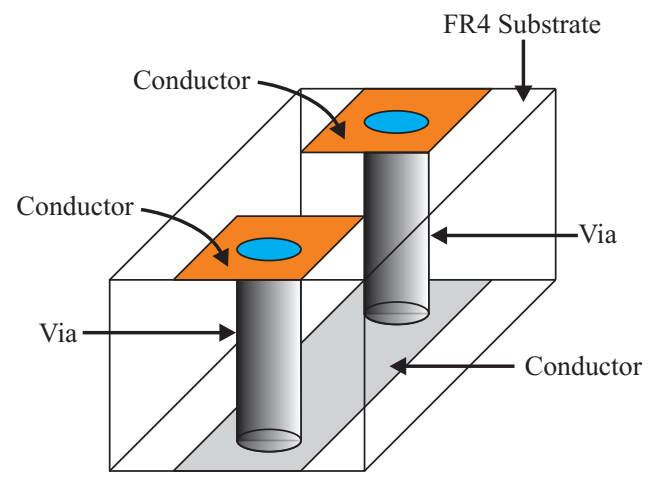

(a)

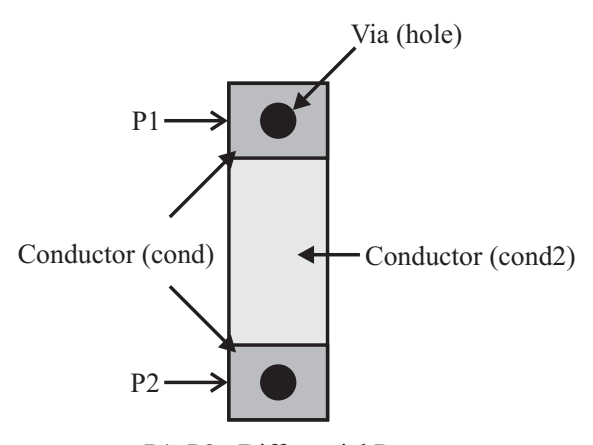

P1, P2 : Differential Ports

(b)

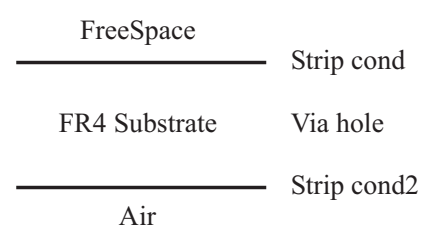

(c)

Figure 52: Illustration of (a) 3-D drawing of CPW bridge modeled in Momentum (b) actual view of CPW bridge modeled in Momentum and (c) substrate characteristics as defined in Momentum.

substrate). The input impedance is given by

$$
Z_{\text {inbridge }}=j Z_{\text {bridge }} \tan \left(\beta \cdot l_{\text {bridge }}\right)
$$

where $Z_{\text {bridge }}$ is the characteristic impedance of the bridge. To determine $Z_{\text {inbridge }}$, the CPW bridge was modeled in Momentum as illustrated in Fig. 52. The vias were modeled with a 6 mil diameter. Two cases were modeled in Momentum: one with a 58 mil substrate, the other with a 25 mil substrate. Figure 53 illustrates $Z_{\text {inbridge }}$ for both cases, obtained via Momentum. The plot clearly illustrates that the CPW bridge over 58 mil FR4 substrate has a fairly high impedance at high frequencies; at $8 \mathrm{GHz} Z_{\text {inbridge }}$ is around $100 \Omega$. Reducing the substrate thickness to 25 mil reduces $Z_{\text {inbridge }}$ to around $45 \Omega$ at $8 \mathrm{GHz}$. In addition, Figure 54 illustrates simulation results obtained from Momentum by modeling the bridge as illustrated in Fig. 52 and varying 
the via diameter. The dimensions of all other components remained the same. It is seen from the Momentum simulation results that increasing the diameter of the via hole lowers the impedance of the bridge.

To understand the effects of the CPW bridges on the performance of the balun, the bridges were modeled in the lumped element model, as illustrated in Fig. 55. The ADS model was modified to include the results in Fig. 53, as illustrated in Fig. 56. Resulting $\mathrm{s}_{21}$ from Momentum (double-y balun in Section 2.3.1 with reduced CPW and CPS transmission lines) and the modified ADS lumped element model are illustrated in Fig. 57. The shifted $\lambda / 8$ resonance frequency from the ADS model agrees very well with the resonance frequency obtained from Momentum for 110 mil balun stub lengths. In addition to the shift in the $\lambda / 8$ resonance, we observe that the bridges amplify the resonance (bridges add significant impedance in series with the stubs causing the junction to become resonant). When the junction becomes resonant, the input impedance is not longer matched at all frequencies. Hence, we would expect to see a large reflection at the $\lambda / 8$ resonance point. This reflection is observed in the $\mathrm{s}_{11}$ data obtained from the ADS and Momentum simulations, illustrated in Fig. 58. The inductance due to the CPW bridges also has the effect of lowering the predicted resonance frequency (near $8 \mathrm{GHz}$ in Fig. 44). Figure 59 compares the $\lambda / 8$ resonance frequency predicted by the ADS model and simulation results from Momentum for 80 mil balun stub lengths. Once again, the lumped element model agrees very well with results obtained from Momentum. We observe a reflection in the $\mathrm{s}_{11}$ data from the ADS and Momentum simulations, illustrated in Fig. 60, at the $\lambda / 8$ resonance point. Both, the ADS and Momentum results agree closely.

Figures 61 and 62 compare results from the ADS model and Momentum for the double-y balun with 110 and 80 mil stub lengths over 25 mil FR4 substrate, respectively. It is seen that the modified lumped element model simulated in ADS agrees better with the Momentum simulations for the baluns over 58 mil FR4 substrate 
than over 25 mil FR4 substrate. This can be attributed to additional parasitics not included in the modified lumped element model in Fig. 55. As the substrate becomes thinner, the height of the CPW bridges decreases, and a parasitic capacitances between the bridge and the conductors become significant. Nevertheless, the modified lumped element model illustrated in Fig. 55 provides insight into the shift of the predicted $\lambda / 8$ resonance.

The balun in Fig. 29 was simulated using Momentum over 25 mil and 58 mil FR4 substrate. Figure 63 illustrates the Momentum simulation results obtained for both cases. It is seen that the $\lambda / 8$ resonance and the CPW resonance are shifted to higher frequencies for the double-y balun over 25 mil FR4 substrate (effective permittivity of the substrate varies with substrate thickness). In addition, the dipole resonance is significantly reduced for the double-y balun over 25 mil substrate, thus illustrating improved performance of the balun junction. This improvement in the performance of the balun junction is expected for the balun over 25 mil FR4 substrate because the CPW bridges better approximate shorted jumpers with the reduced substrate thickness.

From the above results, it can be concluded that the substrate thickness should be minimized when designing the double-y balun, since the impedance of the CPW bridges increases with substrate thickness. It is seen from Fig. 53 that the impedance of the CPW bridge over 58 mil FR4 substrate is around $100 \Omega$ at $8 \mathrm{GHz}$; at high frequencies the bridges act poorly as shorted jumpers. The added impedance of the CPW bridges causes the balun junction to be mismatched and become resonant. Modeling the inductive behavior of the CPW bridges provides insight as to why the shift in the $\lambda / 8$ resonance occurs. 


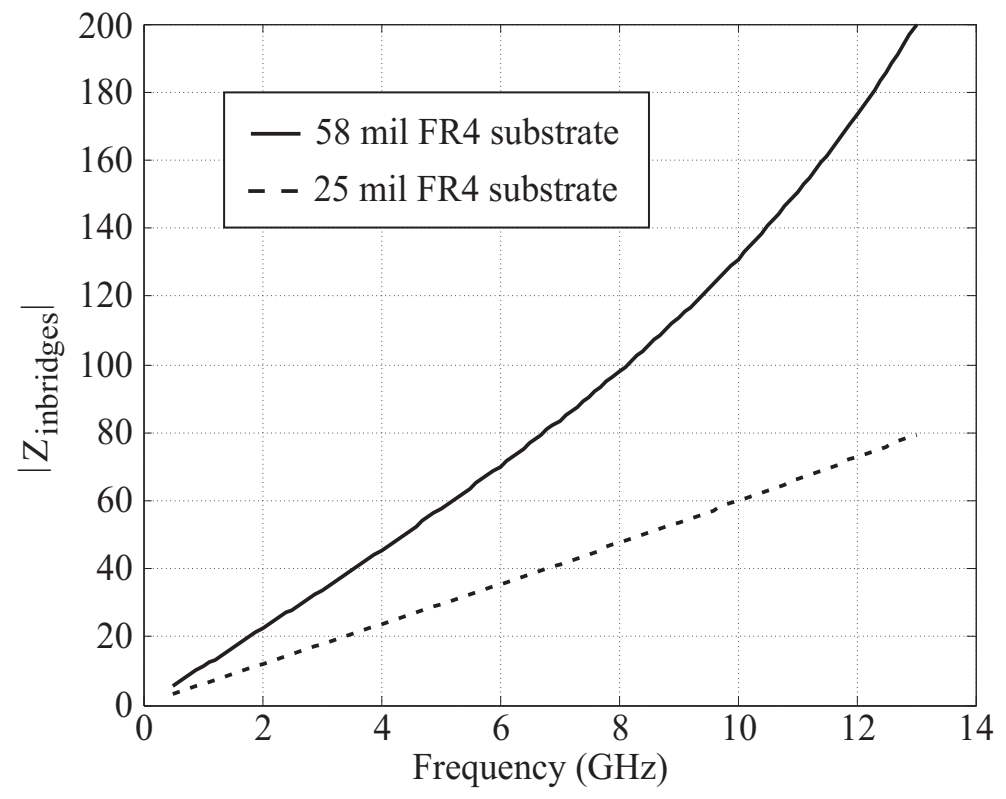

Figure 53: $\mathrm{Z}_{\text {inbridge }}$ computed using Momentum for 25 and 58 mil thick FR4 substrate.

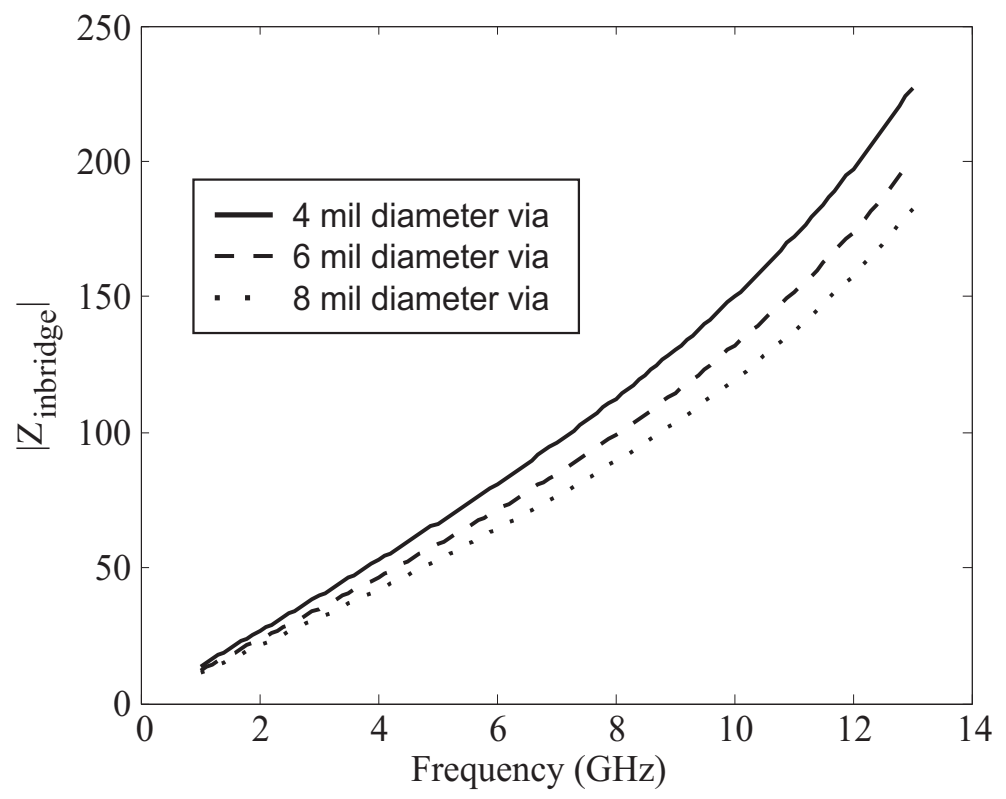

Figure 54: Momentum simulation results illustrating effect of via diameter on CPW bridge impedance. 


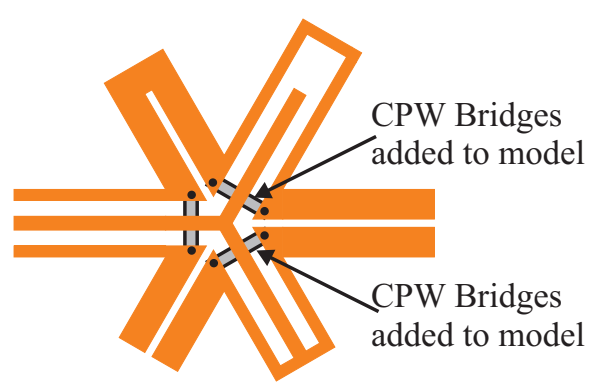

(a)

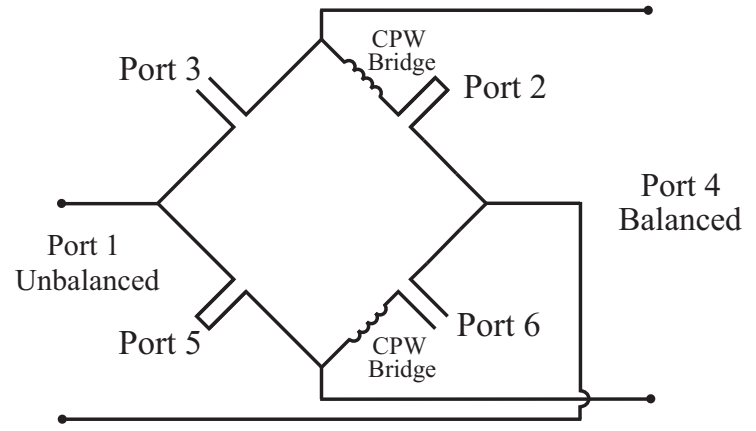

(b)

Figure 55: Modified lumped element model for double-y balun.

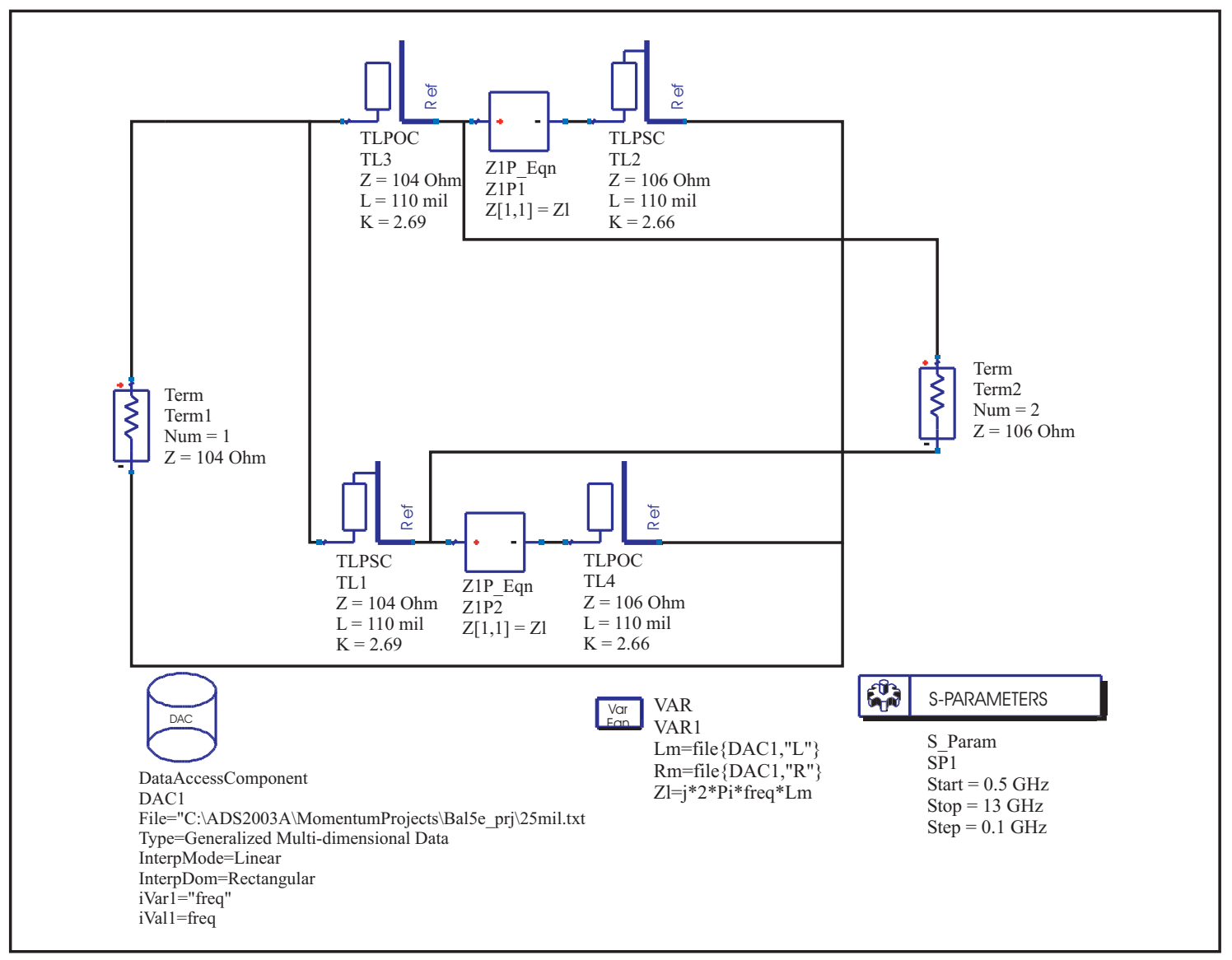

Figure 56: Modified ADS lumped element model for double-y balun. 


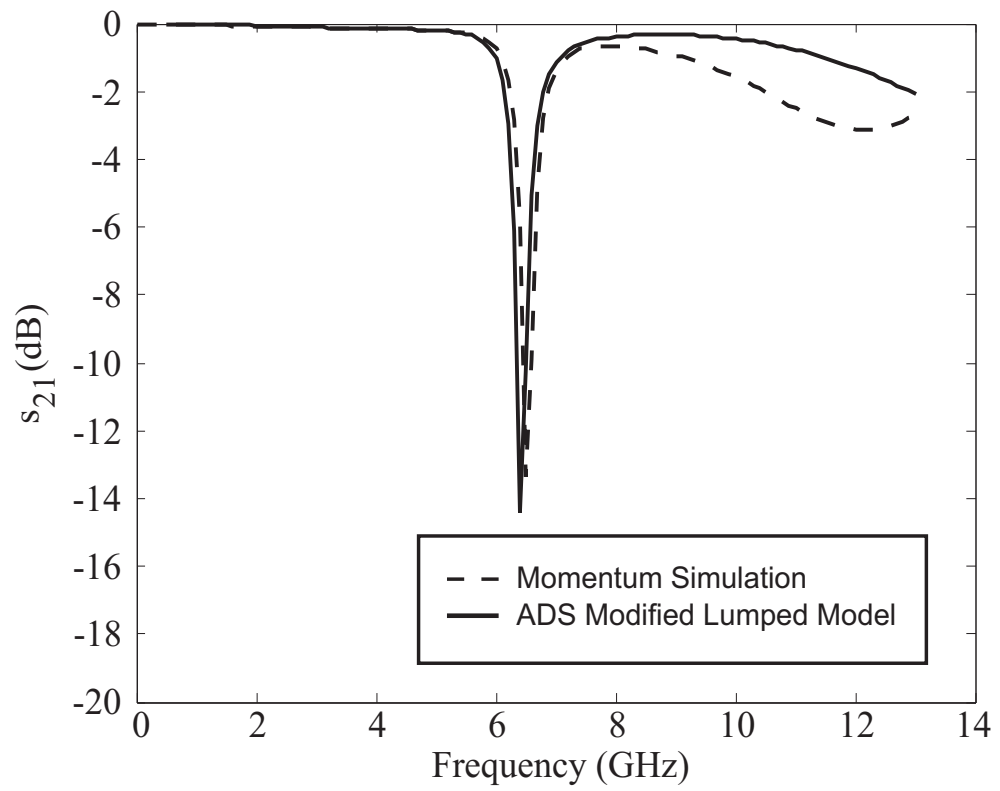

Figure 57: Illustration of shift in $\lambda / 8$ resonance from Momentum and ADS simulations for 58 mil substrate and 110 mil stubs.

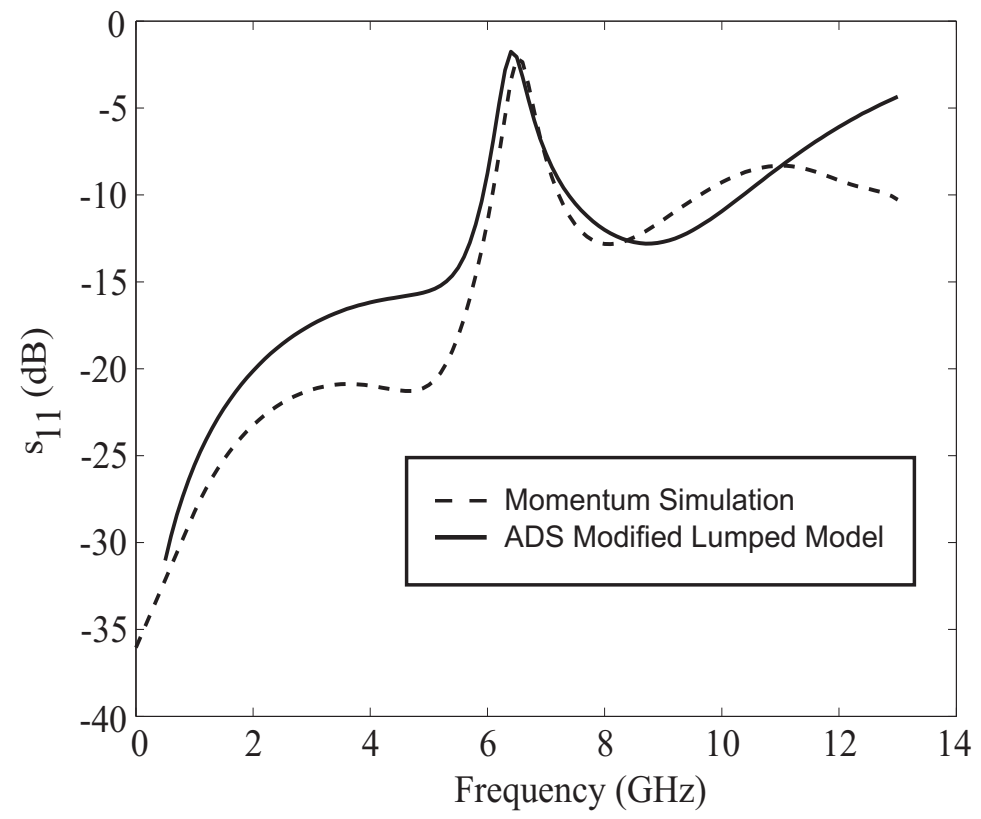

Figure 58: Illustration of reflection at $\lambda / 8$ resonance from Momentum and ADS simulations for 58 mil substrate and 110 mil stubs. 


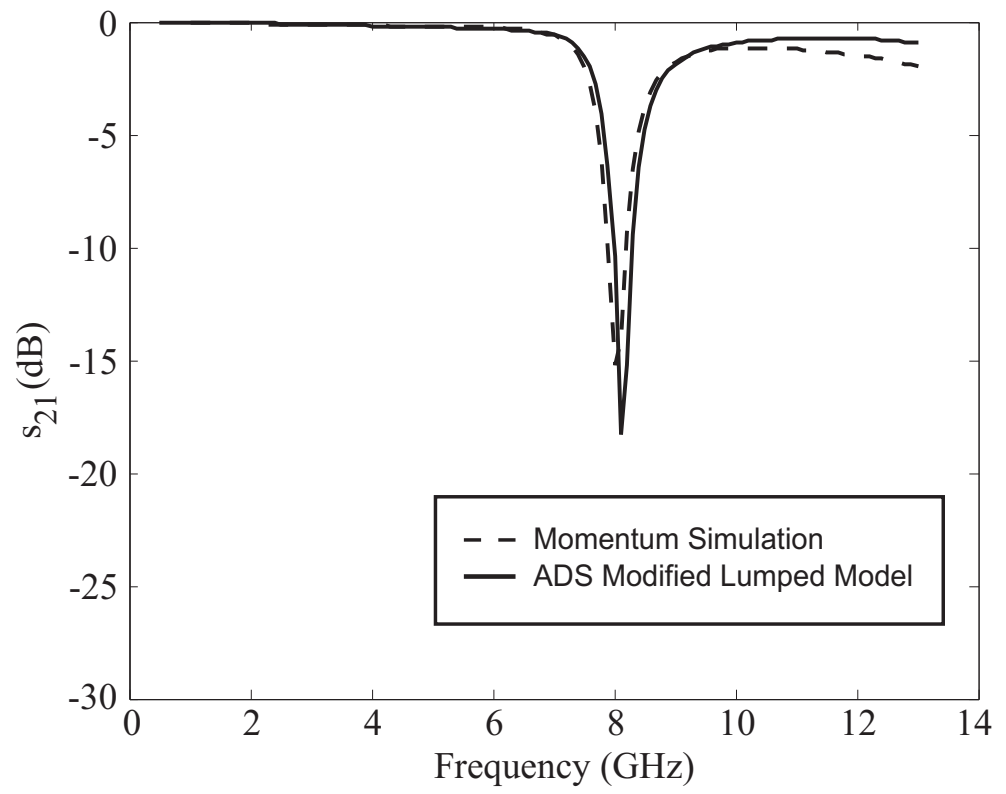

Figure 59: Illustration of shift in $\lambda / 8$ resonance from Momentum and ADS simulations for 58 mil substrate and 80 mil stubs.

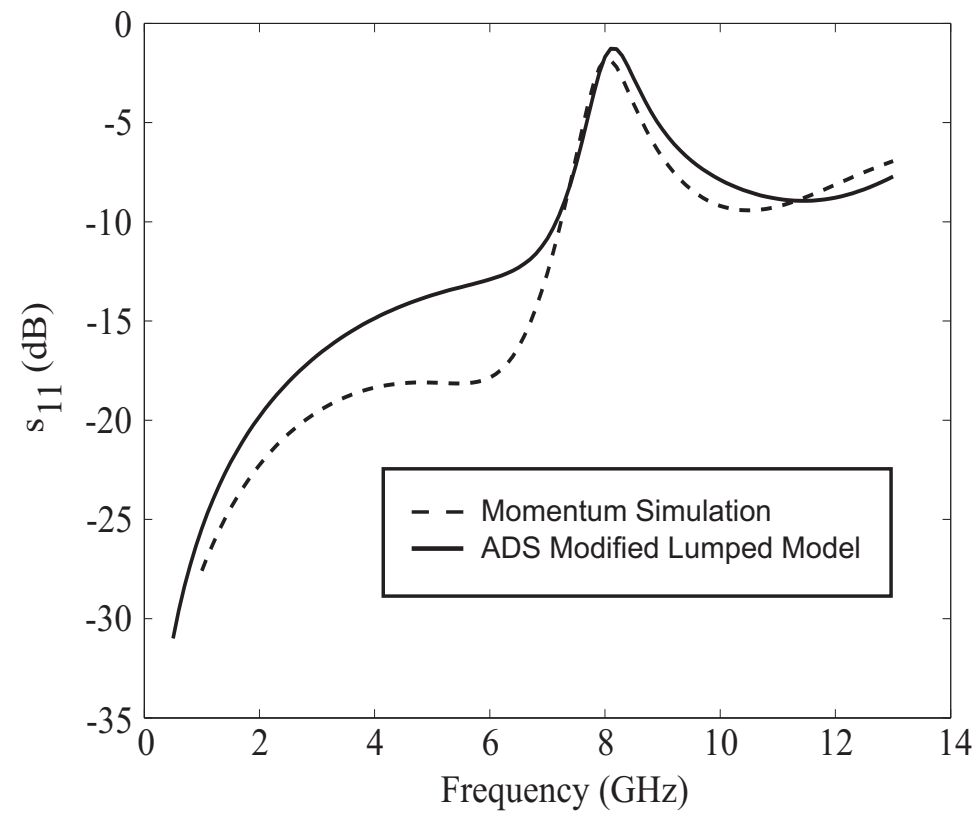

Figure 60: Illustration of reflection at $\lambda / 8$ resonance from Momentum and ADS simulations for 58 mil substrate and 80 mil stubs. 


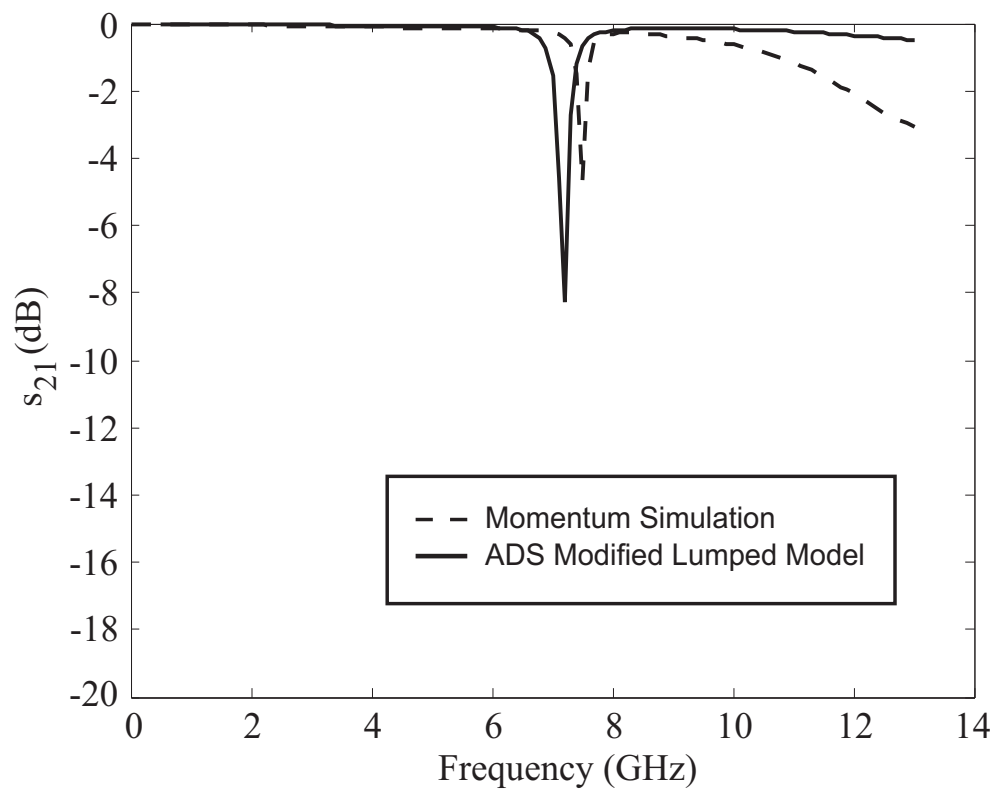

Figure 61: Illustration of shift in $\lambda / 8$ resonance from Momentum and ADS simulations for 25 mil substrate and 110 mil stubs.

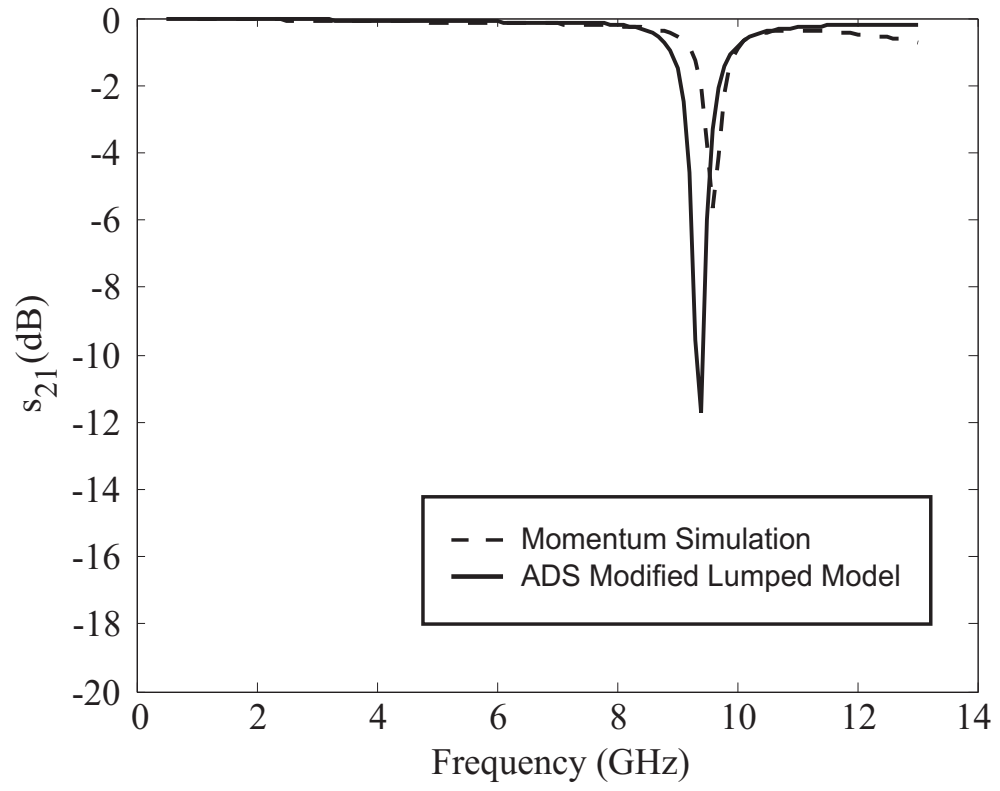

Figure 62: Illustration of shift in $\lambda / 8$ resonance from Momentum and ADS simulations for 25 mil substrate and 80 mil stubs. 


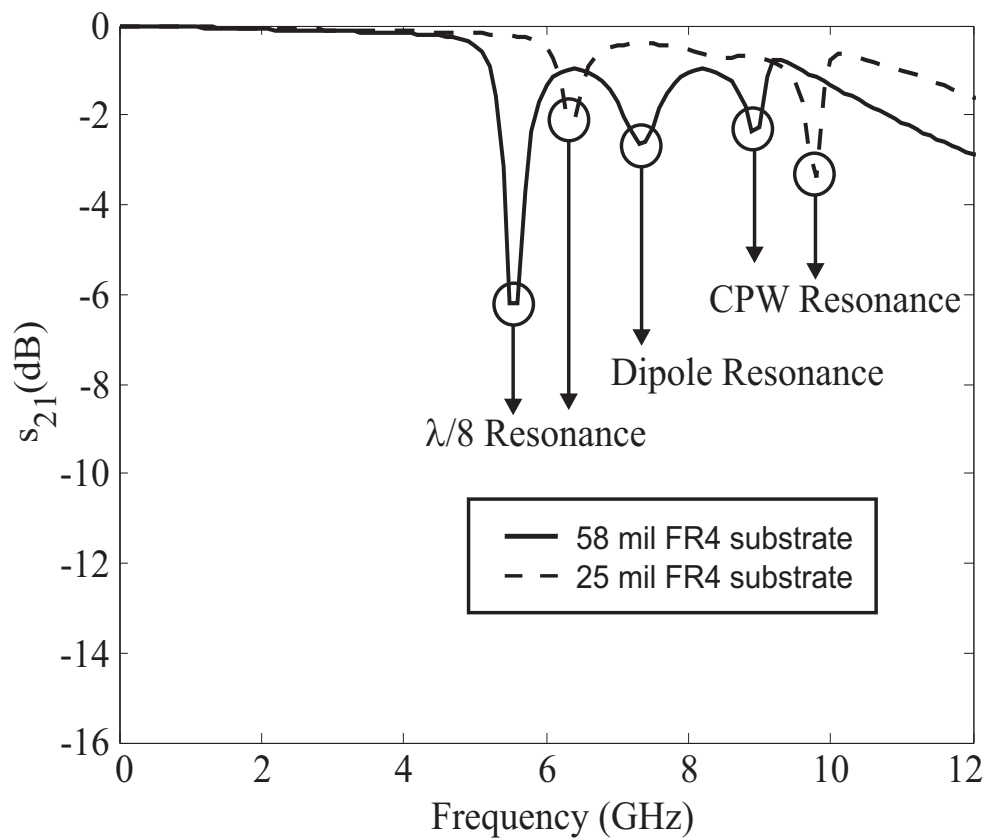

Figure 63: Momentum $\mathrm{s}_{21}$ plot for double-y balun over 25 and 58 mil FR4 substrate with 110 mil CPW and CPS stubs.

\subsection{Aspect Ratio}

The double-y balun in this research is investigated for use with pulsed antennas such as the resistively loaded V-dipole, whose input impedance is around $200 \Omega$. Therefore, the double-y balun is to transition from a $50 \Omega$ coaxial feedline to a $200 \Omega$ antenna. It is difficult to practically achieve a $50 \Omega$ impedance with CPS lines and a 200 $\Omega$ impedance with CPW lines, as explained in 2.1. Also, for the balun to exhibit an all-pass network behavior, the characteristic impedances of the CPW and CPS sections must be equal. The characteristic impedance of the CPW and CPS sections at the balun junction is chosen such that the CPW and CPS lines can be realized practically for the desired impedance. Impedance tapers can then be designed to transition from the coaxial feedline to the balun and from the balun to the antenna. With this design approach, there exists a range of impedance values between the impedance tapers over which several double-y baluns can be designed, each double-y balun having different dimensions, to meet the impedance and realizibility criteria. 
In this section, the performance of two double-y baluns with different aspect ratios is investigated.

The following variables are available for the synthesis of the double-y balun:

$\varepsilon_{r}:$ Substrate material

h: Substrate thickness

$\mathbf{g}_{\text {cps }}:$ CPS gap width

$\mathbf{w}_{c p s}:$ CPS conductor width

$\mathbf{g}_{c p w}:$ CPW gap width

$\mathbf{w}_{c c p w}: \mathrm{CPW}$ center conductor width

$\mathbf{w}_{\text {gcpw }}$ : CPW ground conductor width.

The substrate material is chosen through impedance curves for the CPW and CPS lines, as discussed in 2.1. Once the substrate material is chosen, the smallest substrate thickness available for the material should be chosen (provided the thickness yields adequate mechanical strength), as discussed in the previous section. The CPS section has two parameters, the gap width and the conductor width. To reduce parasitic effects at the balun junction, the overall width of the CPS section $\left(\mathrm{g}_{\text {cps }}+2 \mathrm{w}_{c p s}\right)$ should be minimized. A program was written in Matlab to minimize the overall width of the CPS section, labeled $\mathrm{w}_{\text {cpsout }}$ in Fig. 65, over a range of impedance values. The resulting curve is illustrated in Fig. 64.

The three remaining variables for the synthesis of the balun are the CPW gap width, the CPW center conductor width, and the CPW ground conductor width. From Fig. 65 , it is seen that the $\mathrm{CPW}$ line can be designed by either minimizing $\mathrm{w}_{\text {cpwin }}$ or $\mathrm{w}_{\text {cpwout }}$. Again, a program was written in Matlab to minimize these parameters over a range of impedance values. Figure 64 illustrates the overall width of the $\mathrm{CPW}$ 
and CPS sections over a range of impedance values for various minimization schemes. Two double-y baluns were designed with varying aspect ratios, as illustrated in Fig. 65; $\mathrm{w}_{\text {cpwin }}$ was minimized in one case and $\mathrm{w}_{\text {cpwout }}$ was minimized in the other, and in both cases the overall width of the CPW section was chosen to be equal to the overall width of the CPS section (intersection of the CPS minimization curve with each of the other two curves in Fig. 64). Both baluns were designed on 25 mil FR4 substrate with 80 mil stubs.

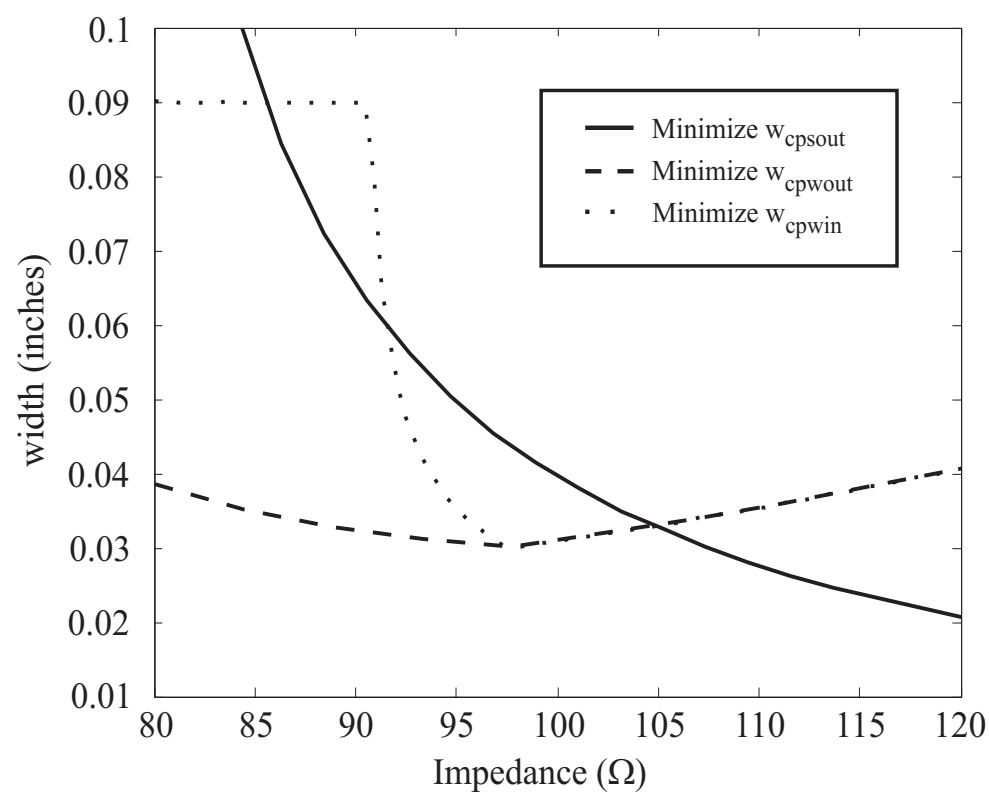

Figure 64: Plot of $\mathrm{w}_{\text {cpwout }}$ for CPS and CPW minimizations.

The double-y baluns illustrated in Figs. 65a and 65b were modeled in Momentum. Figure 66 compares the insertion loss obtained via Momentum for both baluns. It is seen that the insertion loss does not change significantly with the change in aspect ratio. The performances of the baluns cannot be completely characterized by looking at the insertion loss alone, yet it is worthy to note that the insertion loss does not vary dramatically for the two baluns with very different aspect ratios. 


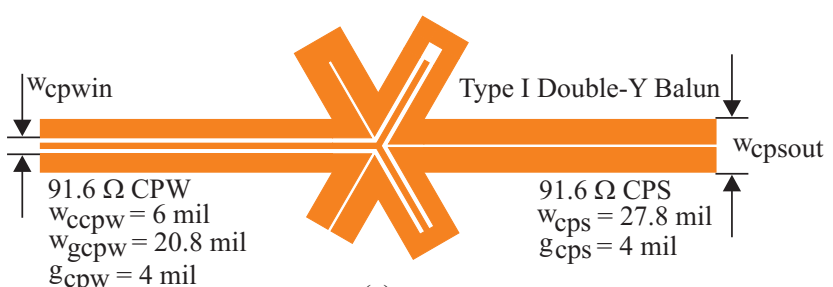

(a)

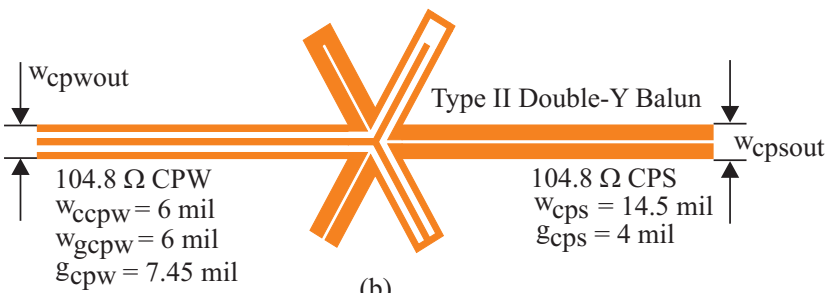

(b)

Figure 65: Double-y balun with (a) $\mathrm{w}_{\text {cpwin }}$ and (b) $\mathrm{w}_{\text {cpwout }}$ minimized.

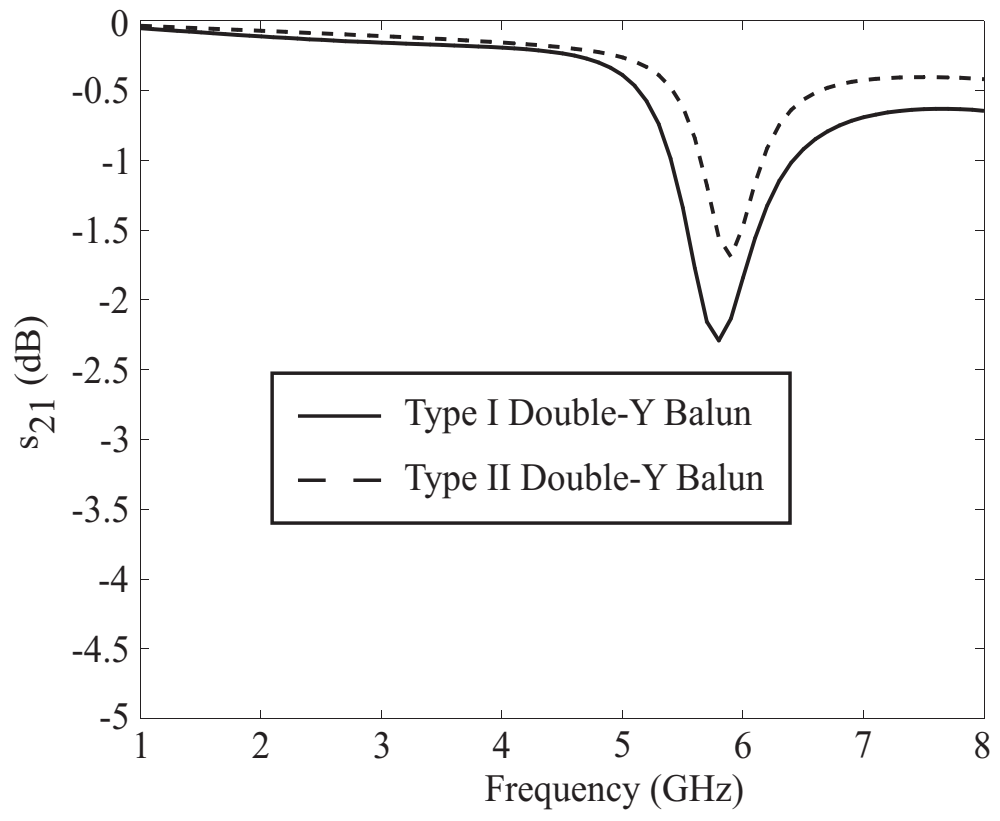

Figure 66: Insertion loss obtained via Momentum simulation of double-y baluns in Fig. 65.

\subsection{Stub Lengths}

\subsubsection{Compensation}

It has been reported in [17] that when designing the double-y balun, the electrical lengths of the open and shorted CPW and CPS stubs, at the balun junction, must be equal. Due to fringing effects the electrical lengths of open and shorted transmission 


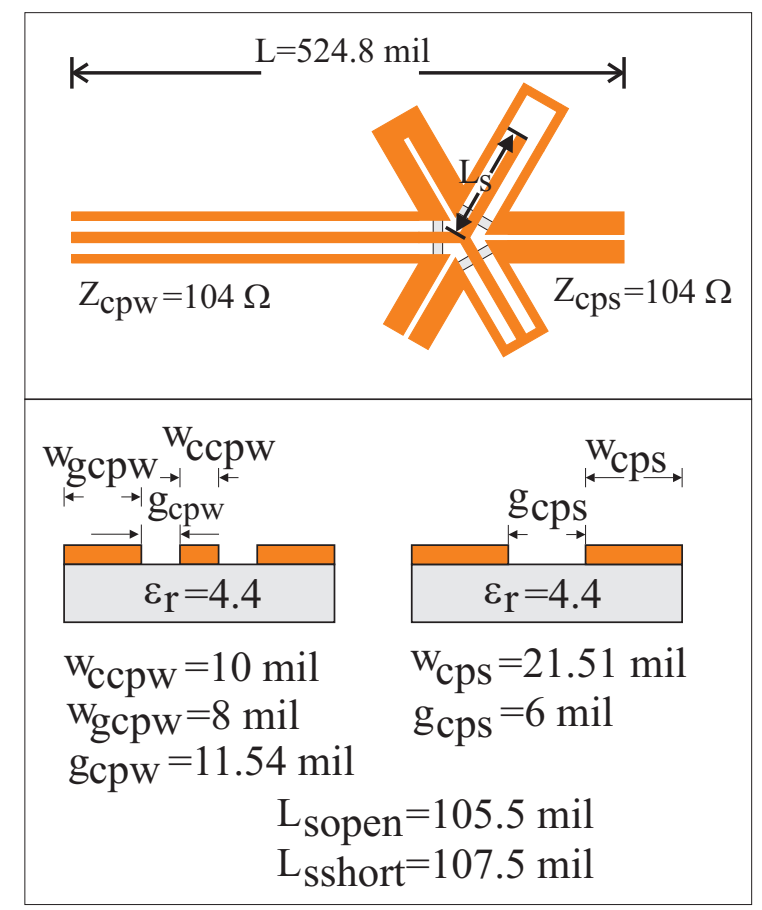

Figure 67: Double-y balun designed with compensated stub lengths.

lines are greater than their physical length; this effect is more pronounced in open transmission lines. Unequal dispersion between CPW and CPS lines is neglected. Approximations for the extension in length of open and shorted lines are available in [23]. The double-y balun in Fig. 29 was re-designed with new stub lengths, as illustrated in Fig. 67.

Figure 68 illustrates Momentum simulation results comparing the balun with and without compensated stubs. It is seen that adjusting the lengths of the stubs to account for fringing, has little effect on the overall performance of the balun. The shift in the $\lambda / 8$ resonance is caused by the shorter stub lengths. While an all-pass behavior can be obtained theoretically for the double-y balun, simulation results indicate that the balun is bandwidth limited.

\subsection{Double-Y Balun Design}

A double-y balun was designed to be manufactured for use in experimental work. Due to manufacturing tolerances, the minimum allowable gap and conductor widths were 


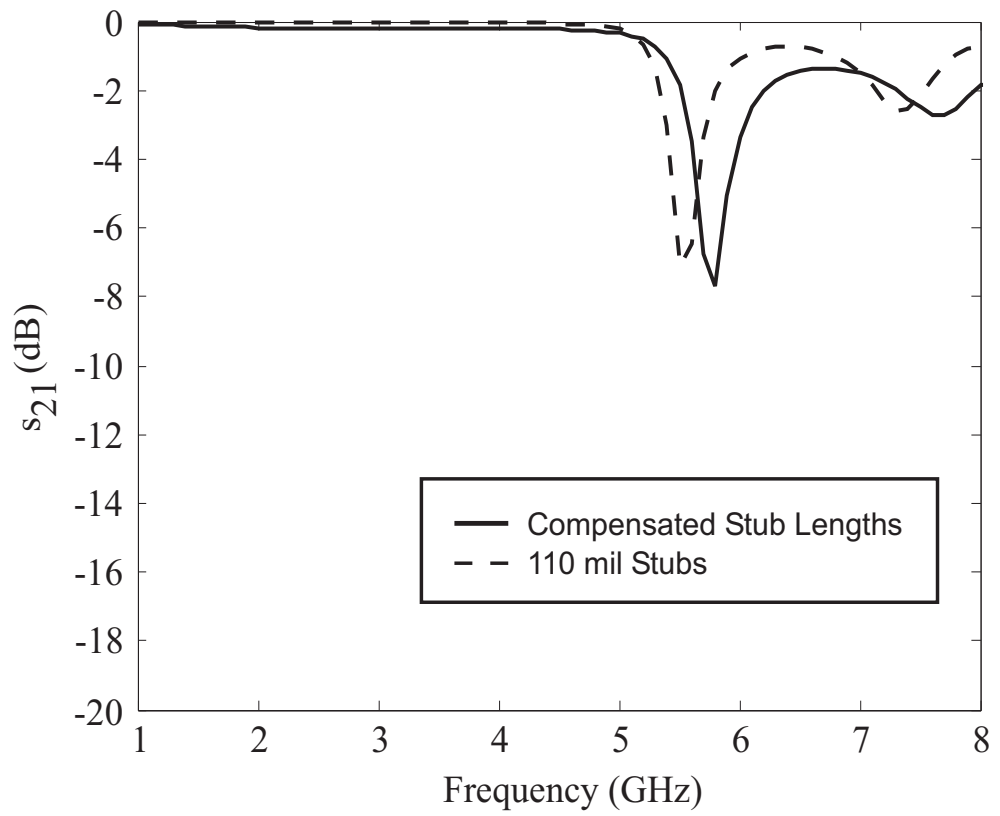

Figure 68: Momentum $\mathrm{s}_{21}$ results for double-y balun with and without compensated stub lengths.

limited to 6 mils. The balun was designed to transition from a $50 \Omega$ coaxial line to a $200 \Omega$ resistively loaded V-dipole [30]. Synthesized values for the gap and conductor widths for the balun are illustrated in Fig. 69. Two versions of the double-y balun were manufactured, one with $80 \mathrm{mil} \mathrm{CPW}$ and CPS stubs, and the other with 110 mil CPW and CPS stubs. Both versions were designed over 25 mil FR4 substrate.

Impedance tapers were added to transition from the $50 \Omega$ coaxial line to the $111 \Omega$ $\mathrm{CPW}$ section of the balun, and to transition from the $111 \Omega$ CPS section of the balun to the $200 \Omega$ antenna feedpoint (the double-y balun cannot transform impedances inherently). Both matching sections were tapered exponentially, with an impedance profile given by

$$
Z(z)=Z_{o} e^{\alpha z}, 0<z<L
$$

where $\alpha$ is given by

$$
\alpha=\frac{1}{L} \ln \left(\frac{Z_{L}}{Z_{o}}\right)
$$

and $Z_{o}$ and $Z_{L}$ are defined as the characteristic impedance at the source and load, respectively [31]. Project specifications limited the lengths of each impedance taper to 


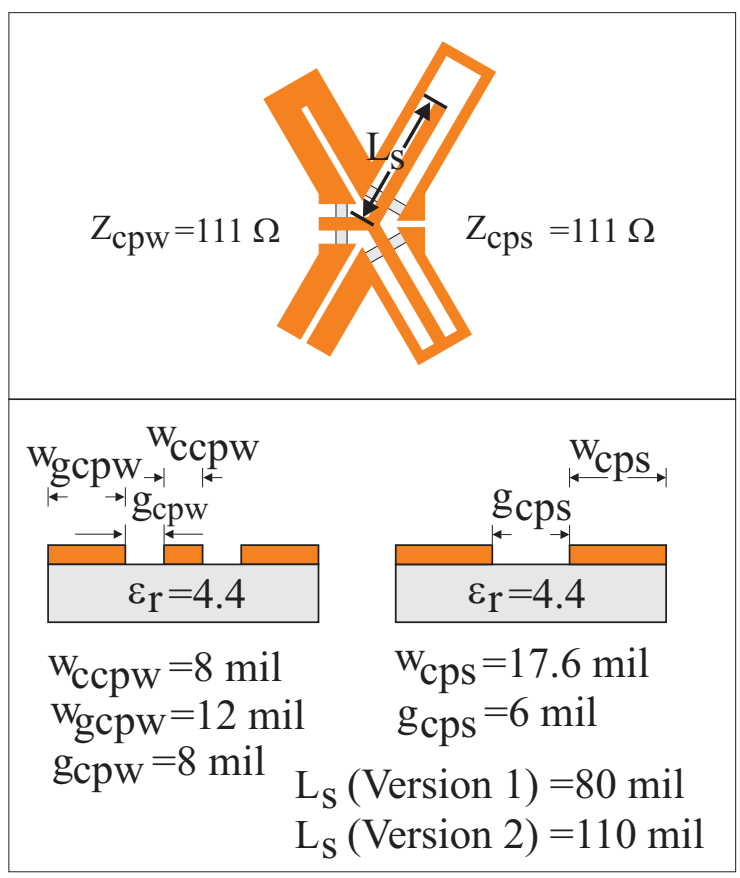

Figure 69: Double-y balun junction designed, over 25 mil FR4 substrate, for use in experimental work.

be 1.6 inches. Figure 70 illustrates the impedance variation along the exponentially tapered CPW section. It is seen that the CPW section starts at $52 \Omega$ instead of $50 \Omega$. This is because the CPW section at the coaxial line to CPW transition was designed as illustrated in Fig. 71. The CPW ground conductors were extended to provide mechanical support for the coaxial line. A slot was provided for fitting the coaxial line (with $2.24 \mathrm{~mm}$ outer diameter). Figure 72 illustrates the synthesized CPW gap and conductor widths along the exponentially tapered section. The CPS section of the double-y balun was designed to taper exponentially from $111 \Omega$, at the balun junction, to $200 \Omega$ at the antenna feedpoint. The impedance profile is illustrated in Fig. 73, where it is seen that the theoretical and synthesized profiles agree exactly. Figure 74 illustrates the exponentially tapered CPS section. The gap was flared towards the antenna feed to increase the impedance as well as to match the physical feed dimensions of the resistively loaded V-dipole. The variation of the gap and conductor widths along the CPS section is illustrated in Fig. 75. 


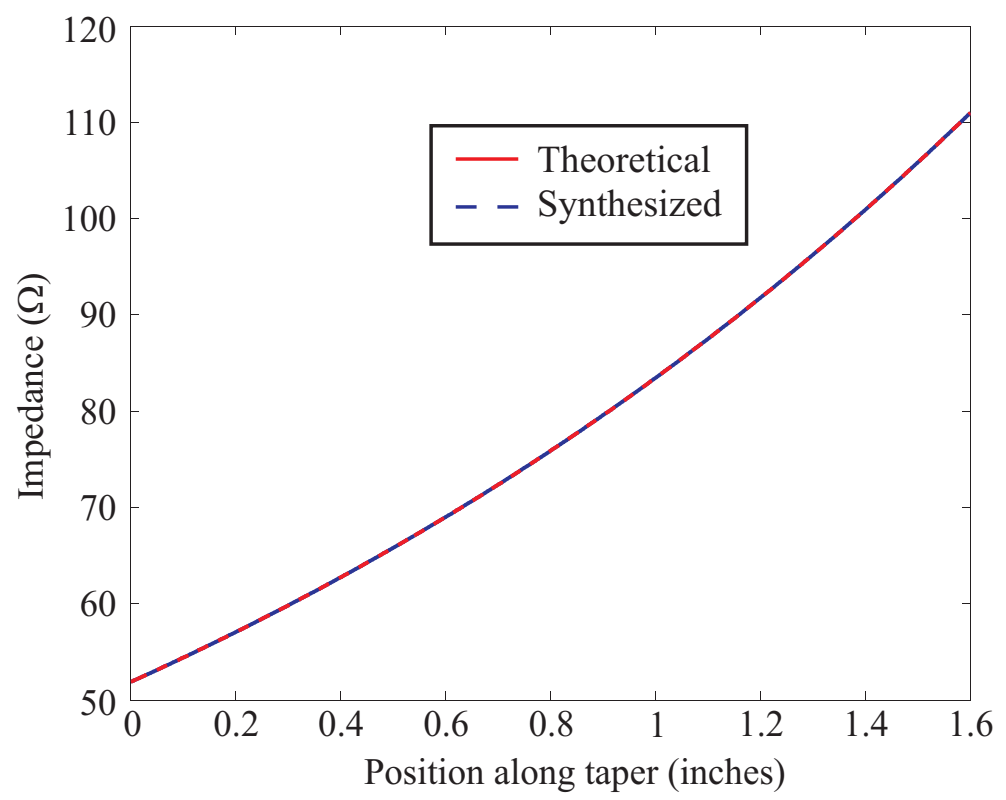

Figure 70: Impedance profile along exponentially tapered CPW section of the double-y balun.

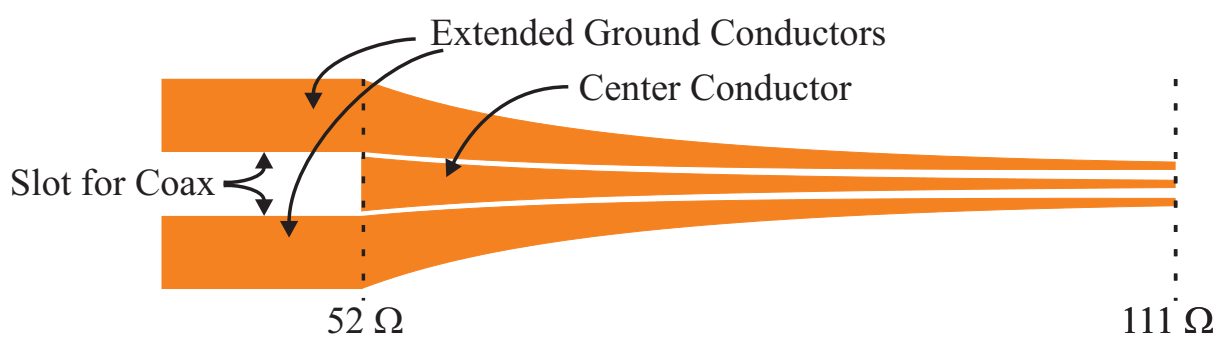

Figure 71: Illustration of CPW tapered section with extended ground conductors at coaxial line to CPW transition. 


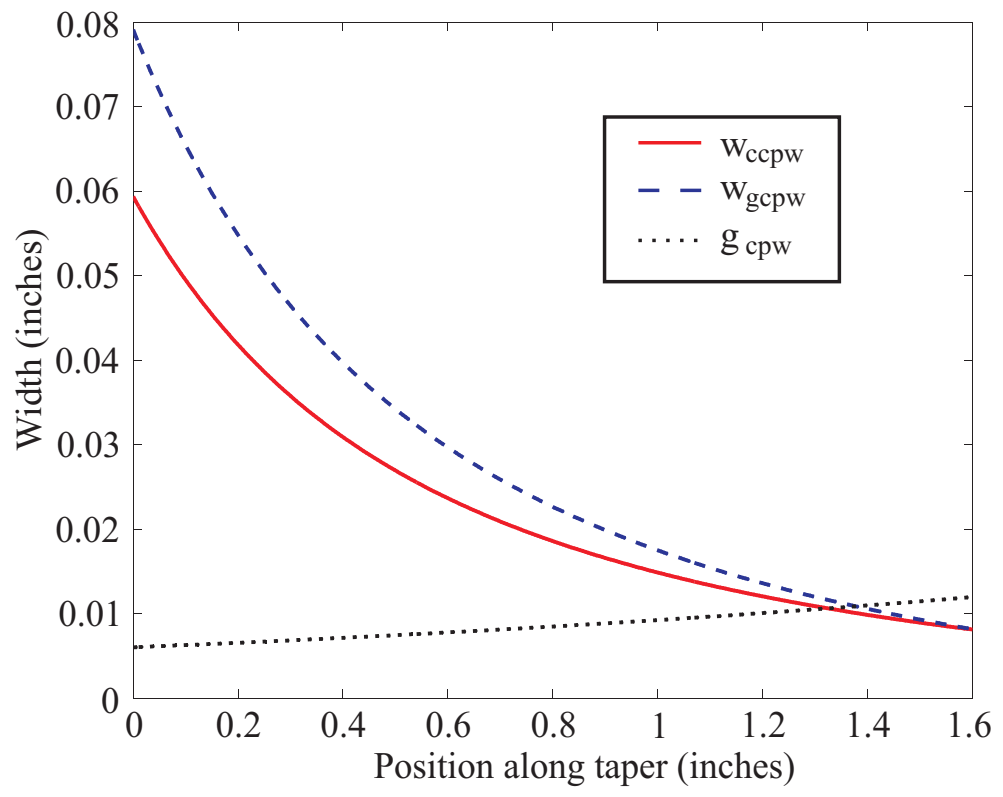

Figure 72: Synthesized CPW gap and conductor widths along exponentially tapered section.

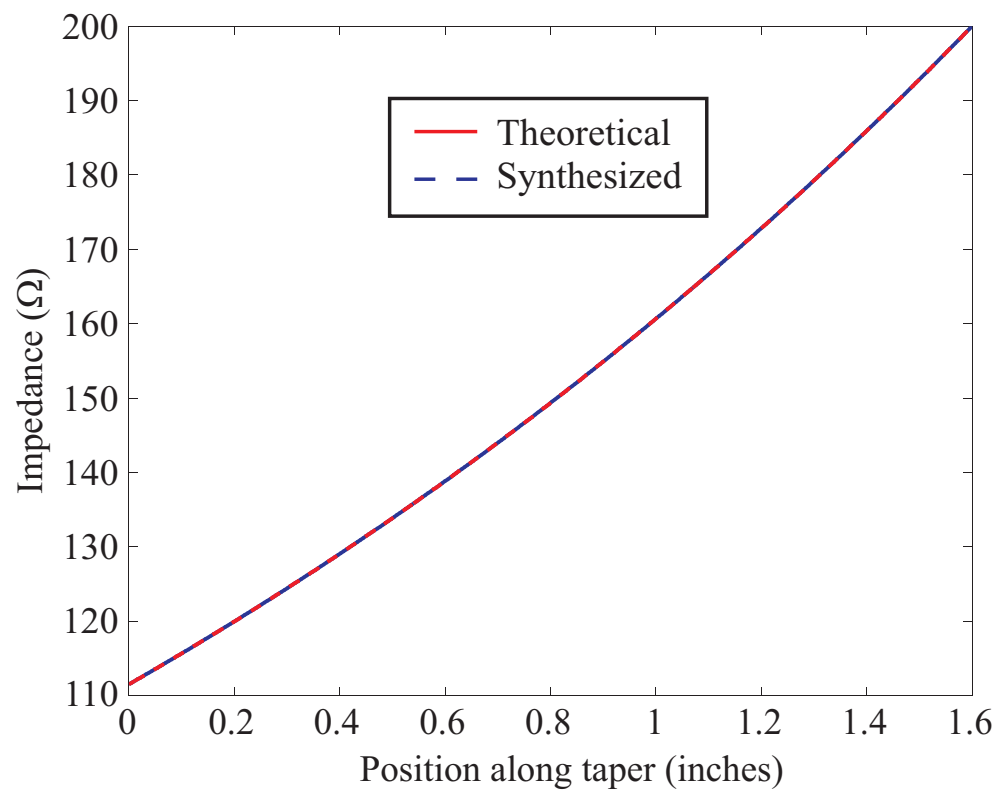

Figure 73: Impedance profile along exponentially tapered CPS section of the doubley balun. 


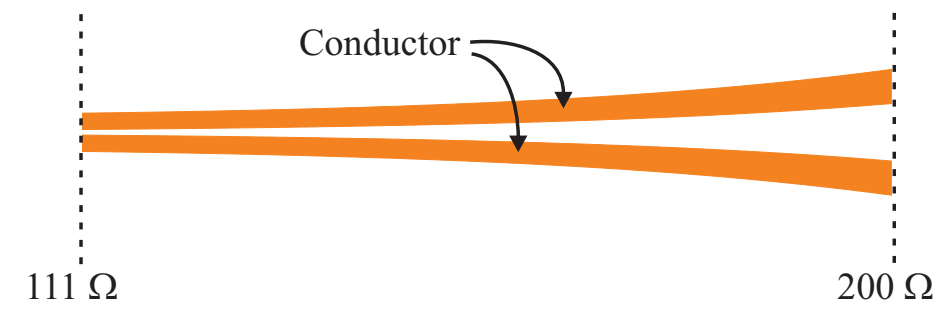

Figure 74: Illustration of exponentially tapered CPS section.

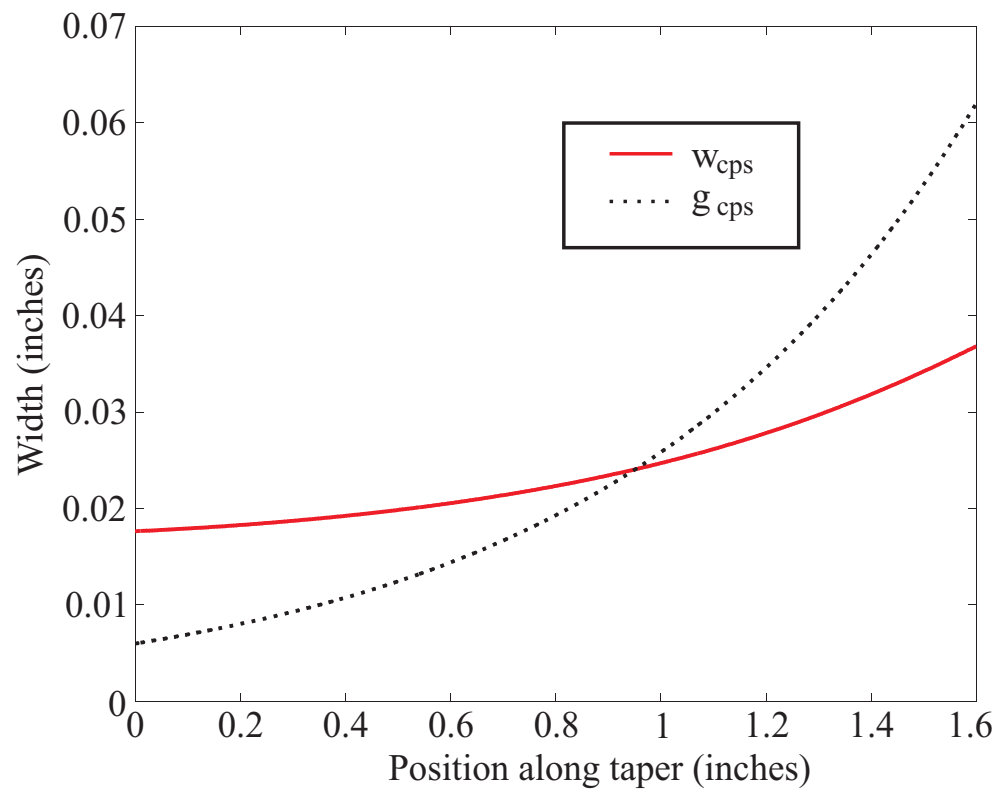

Figure 75: Synthesized CPS gap and conductor widths along exponentially tapered section. 


\section{CHAPTER III}

\section{MODELING BALANCED/UNBALANCED DIPOLE IN NEC}

As discussed in Chapter 1, when a dipole is fed directly with a coaxial line, a commonmode current is induced along the outer conductor of the coaxial line. This induced common-mode current along the outer conductor of the coaxial line causes the feedline to radiate. The double-y balun in this research is investigated for use with pulsed antennas. The dipole is a narrowband antenna, however, it can be modeled numerically with good accuracy and can be constructed with relative ease. Therefore, insight into the effects of an unbalanced feed can be obtained by modeling an unbalanced dipole feed. In this chapter, the effects of an unbalanced dipole feed are investigated using the Numerical Electromagnetic Code (NEC). NEC is a method of moments based electromagnetics code written by the Lawrence Livermore National Laboratory. Currents along the arms and feedline of a balanced vs. unbalanced dipole are examined. Antenna patterns along the azimuth and elevation planes are computed numerically for balanced and unbalanced dipoles.

To better understand the effects of an unbalanced feed on the radiation pattern of an antenna, a $5 \mathrm{~cm}$ dipole and the outer conductor of a coaxial line were modeled using NEC. Constructing a model in NEC serves two purposes: (1) provide a better understanding regarding the effects of an unbalanced feed on the performance of a dipole, and (2) provide a basis to which measured patterns can be compared. The NEC model, along with its equivalent circuit model, is illustrated in Fig. 76. Voltage sources were placed on each arm of the dipole with values of $\alpha$ and 1- $\alpha(\alpha=0.5$ for 
balanced feed and $\alpha=0.1$ for unbalanced feed). The arms of the dipole are balanced when they are driven equally against the feedline; $\alpha=0.5$ models a balanced case. With values of $\alpha \neq 0.5$, the arms of the dipole are no longer balanced; a commonmode current is induced along the feedline thus modeling an unbalanced dipole feed.

Ideally, $\alpha=0$ would represent the worst case for an unbalanced feed, however, NEC does not treat a $0 \mathrm{~V}$ voltage source as a shorted segment. Therefore, the NEC model requires additional modification to model the $\alpha=0$ case; the voltage source with value $\alpha$ has to be removed from the model. This modification becomes necessary when the NEC model is used to study the dipole in the time domain. There is very little difference in the results obtained with $\alpha=0.1$ and $\alpha=0$. Therefore, the NEC model in Fig. 76 is used in this chapter with $\alpha=0.1$ as well as $\alpha=0$ to represent an unbalanced feed. A Wu-King taper, when added to the model in Section 3.3, was placed towards the end of the feedline. Following NEC modeling guidelines, the length of each segment was chosen to be $\lambda / 20$ at the highest frequency, and the radius of the segments were chosen to be $0.0001 \mathrm{~m}$.

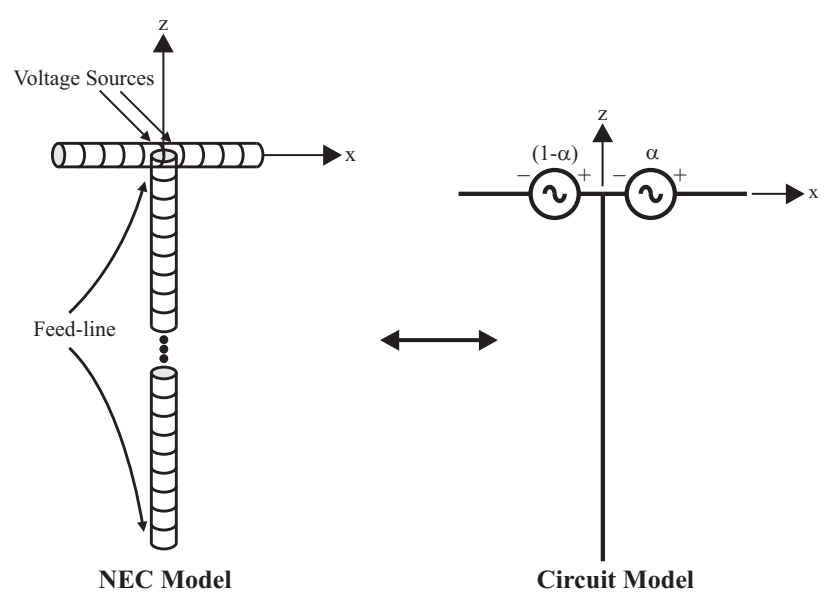

Figure 76: NEC model used to illustrate effects of unbalanced feed on a dipole's radiation pattern. 


\subsection{Balanced/Unbalanced Dipole Currents}

The NEC model in Fig. 76 was used to compute the currents along the arms and feedline of a balanced/unbalanced dipole. The Wu-King loading was not used in the analysis conducted in this section. Figure 77 illustrates the current distribution along the arms of a balanced dipole ( $\alpha=0.5$ in NEC model for voltage sources) at frequencies where the length of the dipole equals $\lambda / 2,3 \lambda / 2$, and $5 \lambda / 2$. The currents are normalized to the maximum value of the current for the $\lambda / 2$ case. At $\lambda / 2$, the current along the arms of the dipole approximates a sinusoidal distribution with its peak at the center of the dipole. The current approaches zero towards the ends of the arms. At $3 \lambda / 2$ and $5 \lambda / 2$, the current distribution along the arms of the dipole has several peaks and nulls before approaching zero towards the ends of the arms.

Figure 78 illustrates the current distribution along the arms of a dipole at $\lambda / 2$ for several values of $\alpha$. The currents are normalized to the maximum value of the current when $\alpha=0.5$. As the arms of the dipole are unbalanced, current is induced along the feedline, thus resulting in unequal currents along the dipole arms. This is also seen to occur at $3 \lambda / 2$ and $5 \lambda / 2$, as illustrated in Figs. 79 and 80 respectively. As the arms of the dipole become unbalanced, the magnitude of the current along one arm of the dipole remains unchanged. However, the magnitude of the current along the other arm of the dipole decreases as the dipole feed becomes more unbalanced; the remaining current is induced along the feedline.

Figure 81 illustrates the current along the feedline for values of $\alpha$. The magnitude of the common-mode current along the feedline increases as the unbalance between the arms of the dipole is increased. For $\alpha=0.5$, the arms of the dipole are balanced, and zero current is induced along the feedline. When the arms of the dipole are unbalanced, the strength of the common-mode current induced along the outer conductor of the coaxial line is dependent on the impedance looking into the feedline. This impedance is dependent on the length of the feedline. This is illustrated in Fig. 82, 
where the current along the feedline is plotted for several feedline lengths, with $\alpha=0.1$ to represent an unbalanced dipole. Maximum current is induced along the feedline for the NEC model in Fig. 76 for odd multiples of a quarter-wavelength. Minimum current is induced along the feed-line for multiples of a half-wavelength.

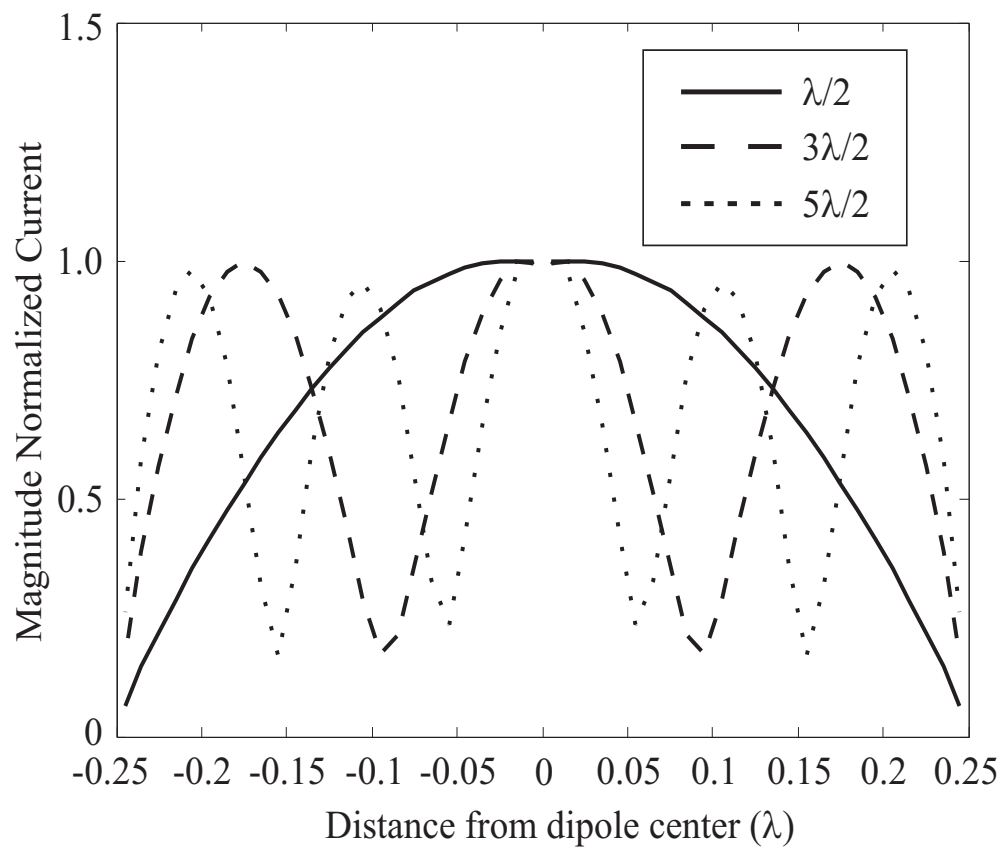

Figure 77: Normalized currents computed using NEC along balanced dipole arms at frequencies where the dipole length is $\lambda / 2,3 \lambda / 2$, and $5 \lambda / 2$. 


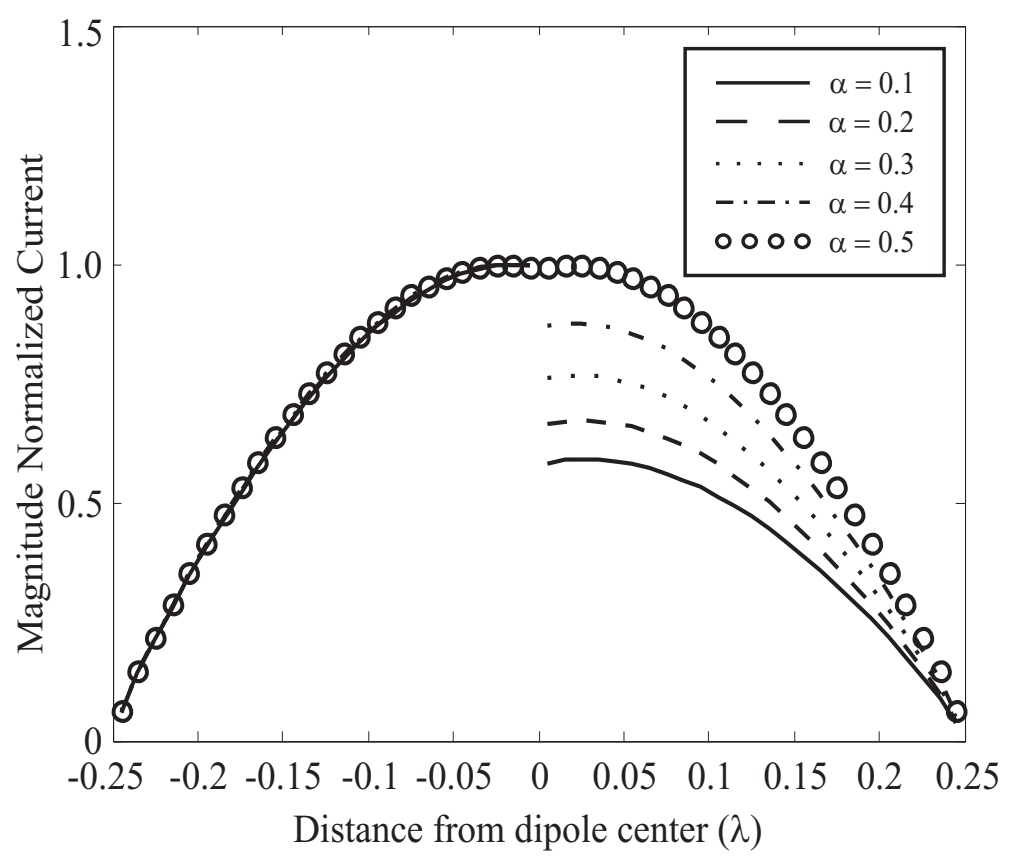

Figure 78: Normalized currents computed using NEC along unbalanced dipole arms of length $\lambda / 2$ and with a feedline length of $5 \lambda / 4$. Currents are normalized to the maximum value obtained with $\alpha=0.5$.

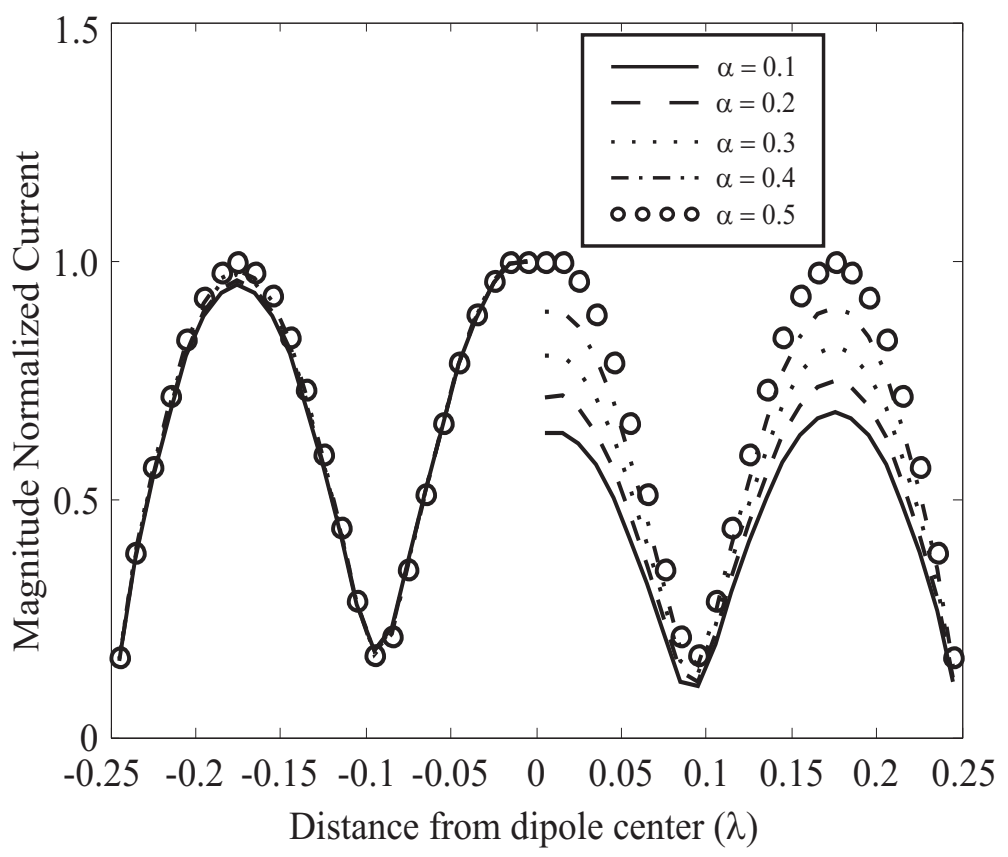

Figure 79: Normalized currents computed using NEC along unbalanced dipole arms of length $3 \lambda / 2$ and with a feedline length of $5 \lambda / 4$. Currents are normalized to the maximum value obtained with $\alpha=0.5$. 


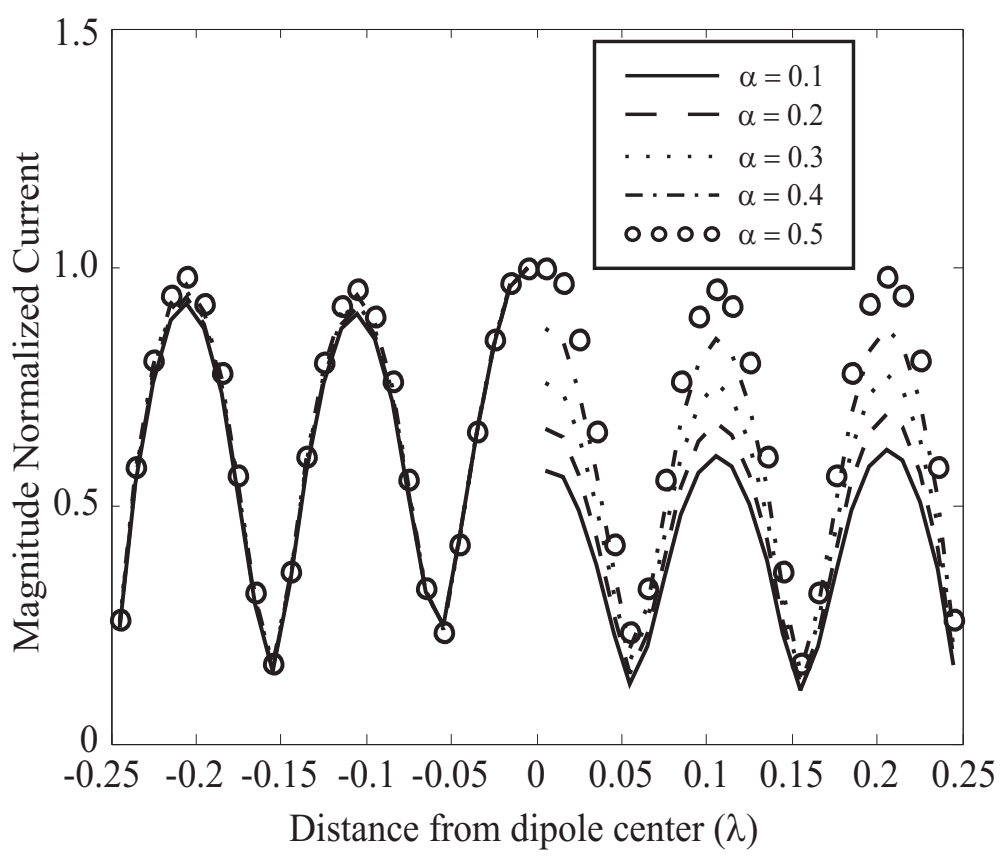

Figure 80: Normalized currents computed using NEC along unbalanced dipole arms of length $5 \lambda / 2$ and with a feedline length of $5 \lambda / 4$. Currents are normalized to the maximum value obtained with $\alpha=0.5$.

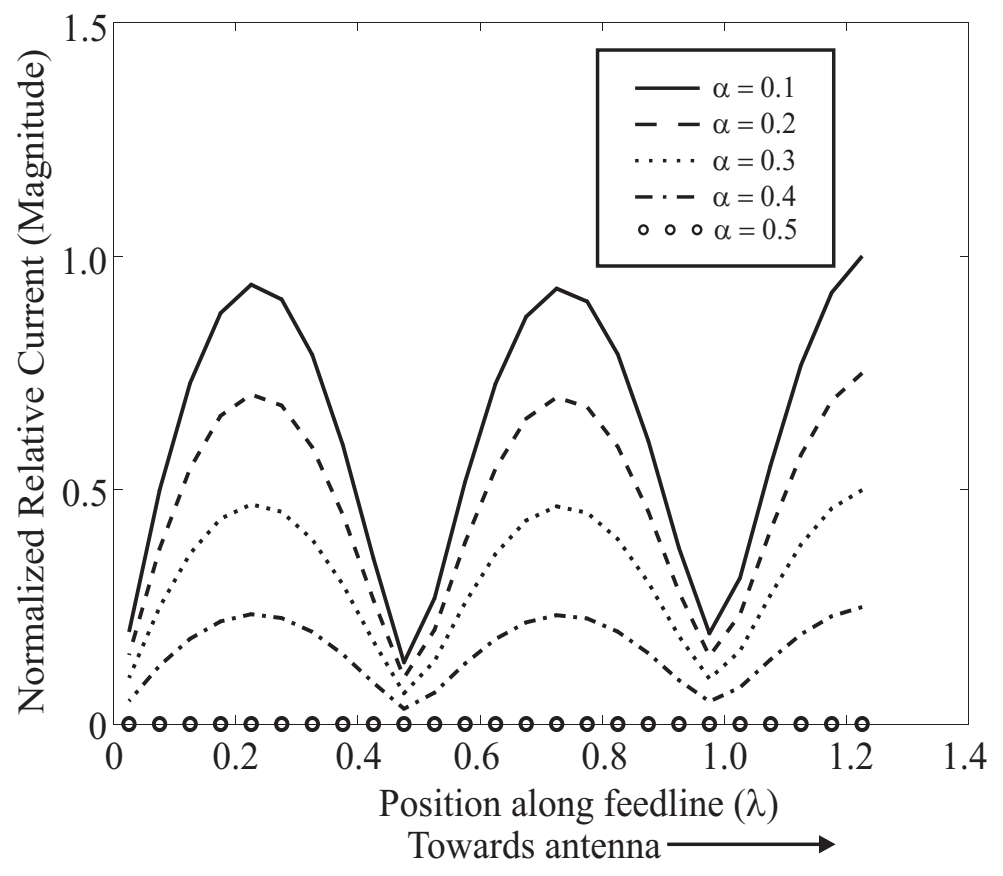

Figure 81: Normalized relative currents computed using NEC along feedline of unbalanced dipole for values of $\alpha$. Currents are normalized to the maximum value obtained with $\alpha=0.5$. 


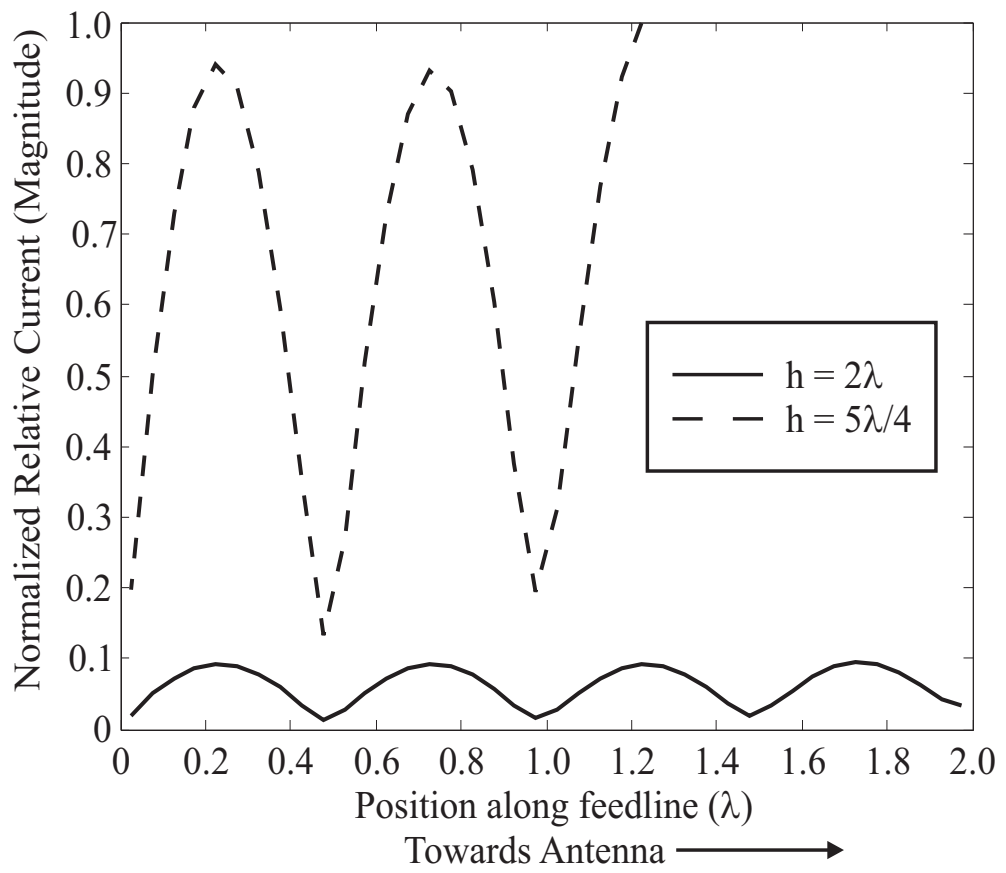

Figure 82: Normalized relative currents computed using NEC along feedline, with height $h$, of unbalanced dipole. Currents are normalized to the maximum value obtained with $\mathrm{h}=5 \lambda / 4$. 


\subsection{Dipole Amplitude Patterns}

A 3-D normalized far field amplitude pattern of a balanced dipole, with arms oriented along the $\mathrm{x}$-axis, is illustrated in Fig. 83a. This is the familiar donut pattern of a balanced dipole. As seen from the 3-D pattern, there is a null in the pattern along the axis of the dipole arms. Normalized amplitude patterns were generated along the azimuth and elevation planes (these planes are defined in Fig. 84) for a balanced dipole using the NEC model illustrated in Fig. 76 without Wu-King loading. The magnitude of the $\mathrm{E}_{\phi}$ component at the far field is plotted along the azimuth plane, and the magnitude of the $\mathrm{E}_{\theta}$ component at the far field is plotted along the elevation plane. Figure 85 illustrates the resulting normalized amplitude patterns for a balanced dipole computed using the NEC model. It is seen that the pattern for a balanced dipole is symmetric in each plane and identical but rotated in both planes.

A 3-D normalized far field pattern of an unbalanced dipole, with arms oriented along the $\mathrm{x}$-axis, is illustrated in Fig. 83b. The pattern was generated using the NEC model in Fig. 76 with $\alpha=0.1$ and a feedline length of $11 \lambda / 4$. The unbalanced feed clearly distorts the desired donut pattern of the dipole. Furthermore, it is seen that the unbalanced dipole radiates strongly towards the feedline. As illustrated in Figs. 85a, 87a, and 86a, unbalancing the arms of a dipole (with $\alpha=0.1$ ) has little effect on the normalized amplitude patterns along the azimuth cut at $\lambda / 2$. Figure 88 compares the normalized amplitude pattern of a balanced dipole vs. that of an unbalanced dipole at $\lambda / 2$. It is clear that it is difficult to discern between the azimuth pattern of a balanced dipole and an unbalanced dipole at $\lambda / 2$. Therefore, when measuring these patterns experimentally, an accurate measurement setup is required; there are no significant differences in the shape of the azimuth pattern between the balanced and unbalanced dipole at $\lambda / 2$ so the errors introduced in the measurement setup must be minimal (e.g. error in alignment of the dipoles, error in alignment with the positioner, presence of clutter, etc.). 
Unlike the azimuth patterns of a dipole at $\lambda / 2$, the azimuth pattern of a balanced dipole at $3 \lambda / 2$ differs significantly from that of an unbalanced dipole at $3 \lambda / 2$. This is illustrated in Figs. 85b, 87b, and 86b. The azimuth pattern of a balanced dipole at $5 \lambda / 2$ differs significantly from that of an unbalanced dipole at $5 \lambda / 2$. This is illustrated in Figs. 85c, 87c, and 86c. As illustrated previously in Fig. 77, the current distribution for the $\lambda / 2$ dipole approximates a sinusoidal waveform with a peak at the center of the dipole and nulls at the ends of the dipole. Unbalancing the currents along the dipole arms at $\lambda / 2$ has little effect on the resulting pattern along the azimuth plane. However, unbalancing the currents along the dipole arms at $3 \lambda / 2$ and $5 \lambda / 2$ affects the resulting azimuth patterns considerably.

As seen from Figs. 87 and 86, the normalized amplitude pattern is significantly affected along the elevation cut when the dipole is unbalanced. This is caused by the current distribution along the feedline. Furthermore, it is seen that the pattern along the elevation plane for an unbalanced dipole is dependent on the length of the feedline, since the current distribution varies with the length of the feedline. The pattern along the elevation plane for an unbalanced dipole with a $5 \lambda / 4$ length feedline differs significantly from the elevation pattern of an unbalanced dipole with a $11 \lambda / 4$ length feedline, as illustrated in Figs. 87 and 86.

Figure 89 illustrates azimuth patterns $\left(\mathrm{E}_{\phi}\right.$ component) generated using the NEC model in Fig. 76, as a function of $\alpha$. As seen from Fig. 89a, it is difficult to discern the change in the pattern at $\lambda / 2$ as a function of $\alpha$. A more significant change in the azimuth pattern, as a function of $\alpha$, is observed in the patterns at $3 \lambda / 2$ and $5 \lambda / 2$, as illustrated in Figs. 89b and 89c, respectively.

Figure 90 illustrates elevation patterns ( $\mathrm{E}_{\theta}$ component) generated using the NEC model in Fig. 76, as a function of $\alpha$. The patterns were generated with a $11 \lambda / 4$ length feedline. The variation in the patterns at $\lambda / 2,3 \lambda / 2$, and $5 \lambda / 2$ is clearly seen as a function of $\alpha$. Figures 89 and 90 illustrate the sensitivity of the normalized patterns 
to variations in $\alpha$.

From the amplitude patterns generated using the NEC model, it is seen that unbalancing the arms of a half-wavelength dipole has little effect on the pattern along the azimuth plane at $\lambda / 2$. However, at higher frequencies, the pattern along the azimuth plane is affected considerably. The elevation patterns of a balanced dipole differ significantly from those of an unbalanced dipole, even at $\lambda / 2$. The NEC patterns for the balanced and unbalanced dipole provide us with insight into how to measure the patterns of balanced and unbalanced dipoles effectively: (1) an accurate measurement setup is required, since there is little difference between the azimuth patterns at $\lambda / 2,(2)$ in addition to the azimuth patterns at $\lambda / 2$, azimuth and elevation patterns at $3 \lambda / 2$ and $5 \lambda / 2$ can be measured to determine whether the arms of a dipole are balanced, and (3) an accurate measurement setup is required to measure the elevation patterns, since these patterns are dependent on the length and position of the radiating feedline; movement in the position of the coaxial feedline could alter the measured data. Radiation from the feedline of an unbalanced dipole must somehow be confined. 

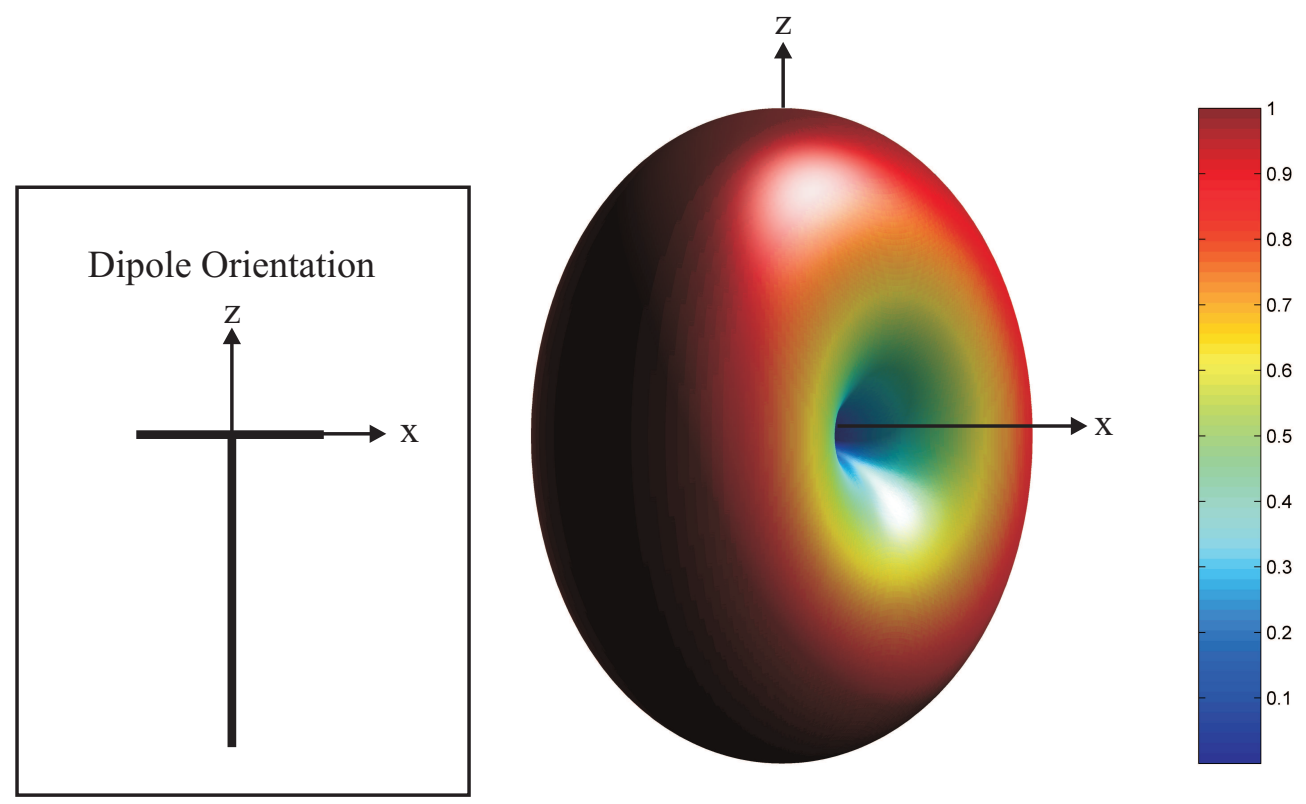

(a)
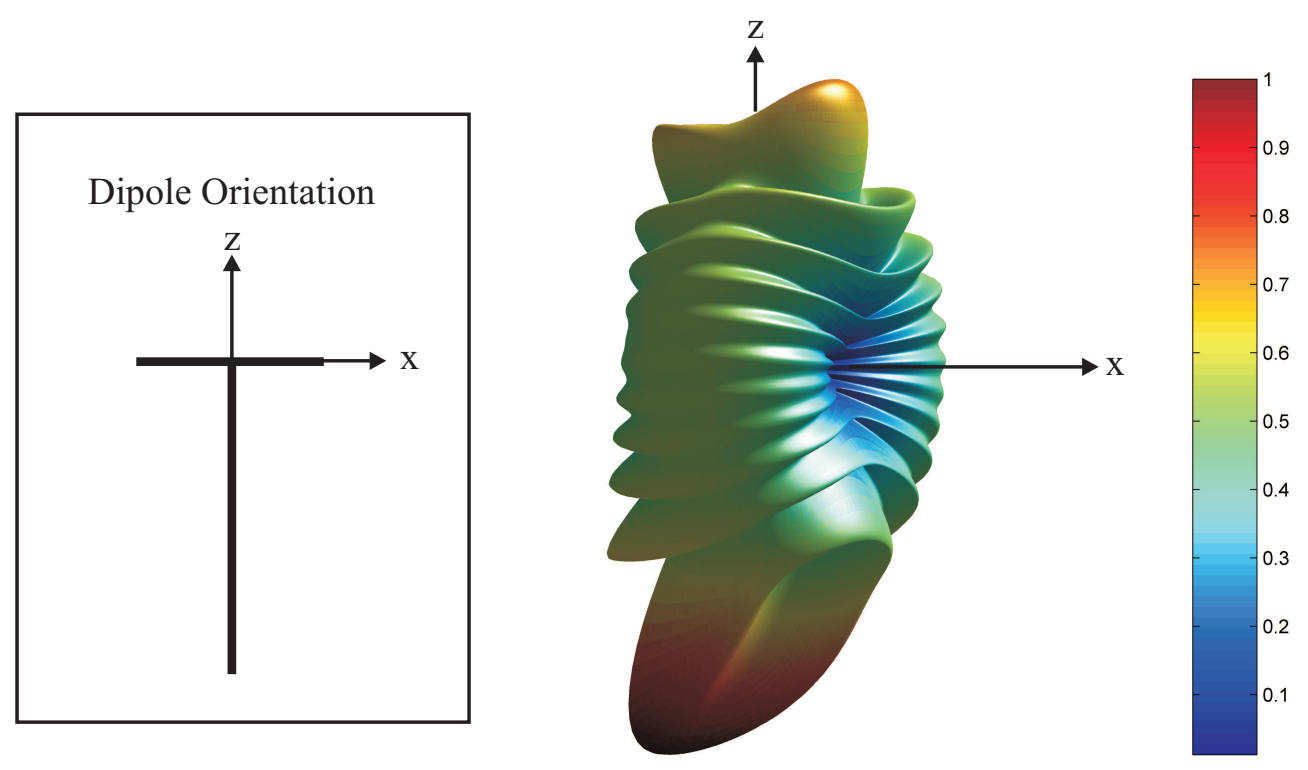

(b)

Figure 83: 3-D normalized far field pattern, generated with NEC model in Fig. 76, of (a) balanced dipole with $\alpha=0.5$ and (b) unbalanced dipole with $\alpha=0.1$. 


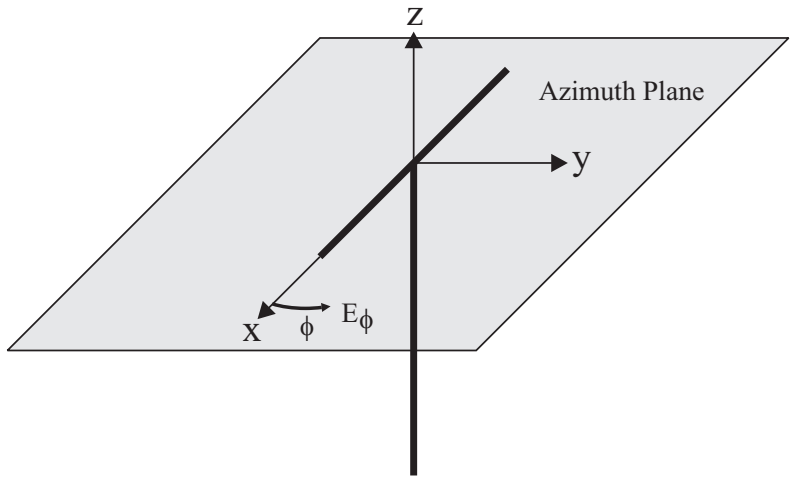

(a)

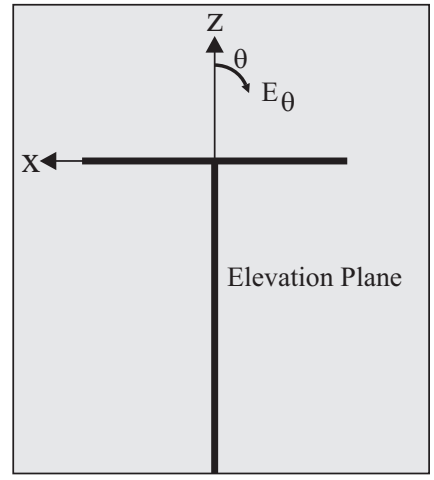

(b)

Figure 84: Illustration of (a) azimuth plane and (b) elevation plane with $\phi$ and $\theta$ defined in spherical coordinates. 


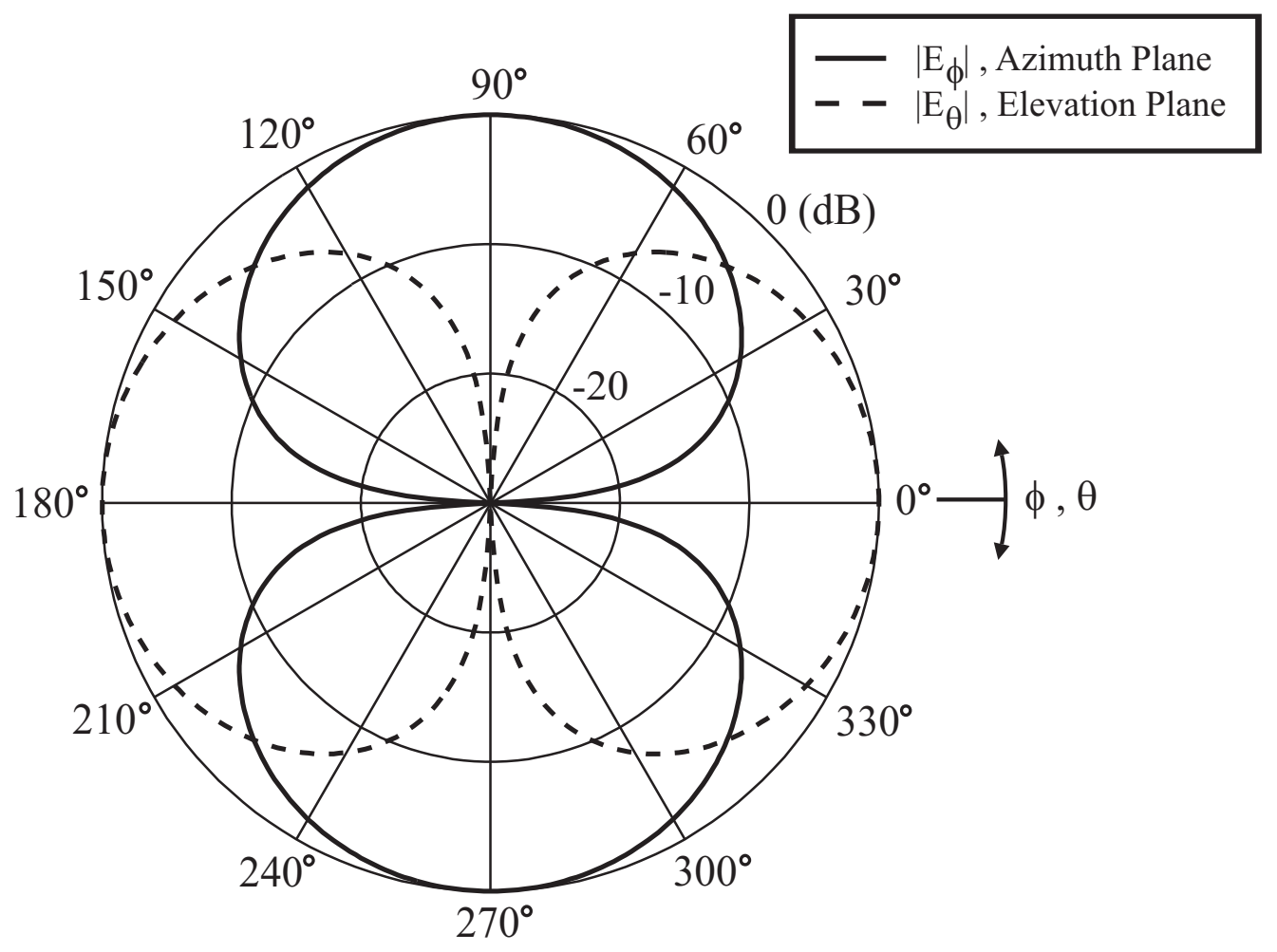

(a)

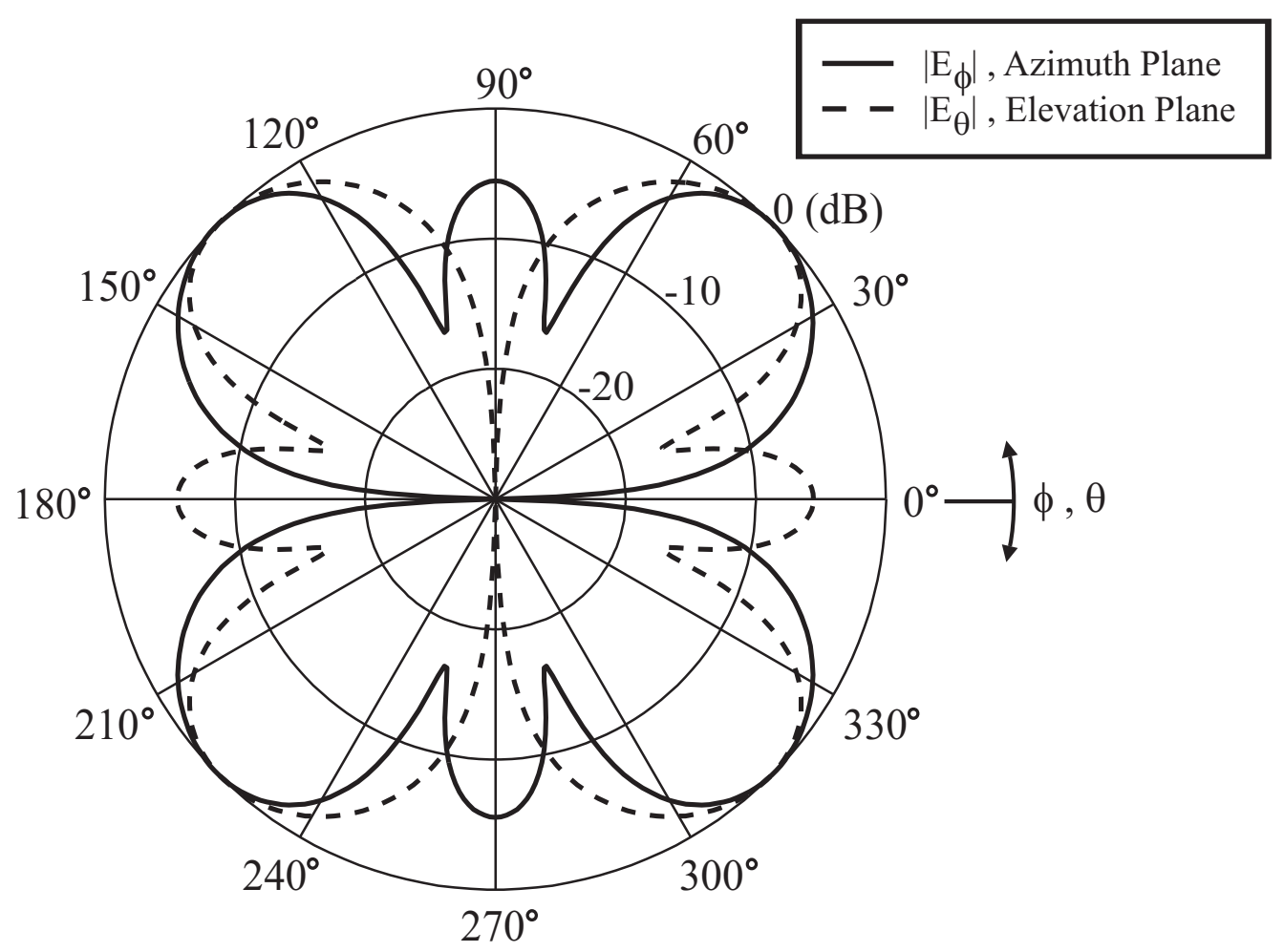

(b)

Figure 85: Normalized amplitude patterns for balanced dipole at (a) $\lambda / 2$, (b) $3 \lambda / 2$, and (c) $5 \lambda / 2$ computed using the NEC model in Fig. 76 with $\alpha=0.5$. 


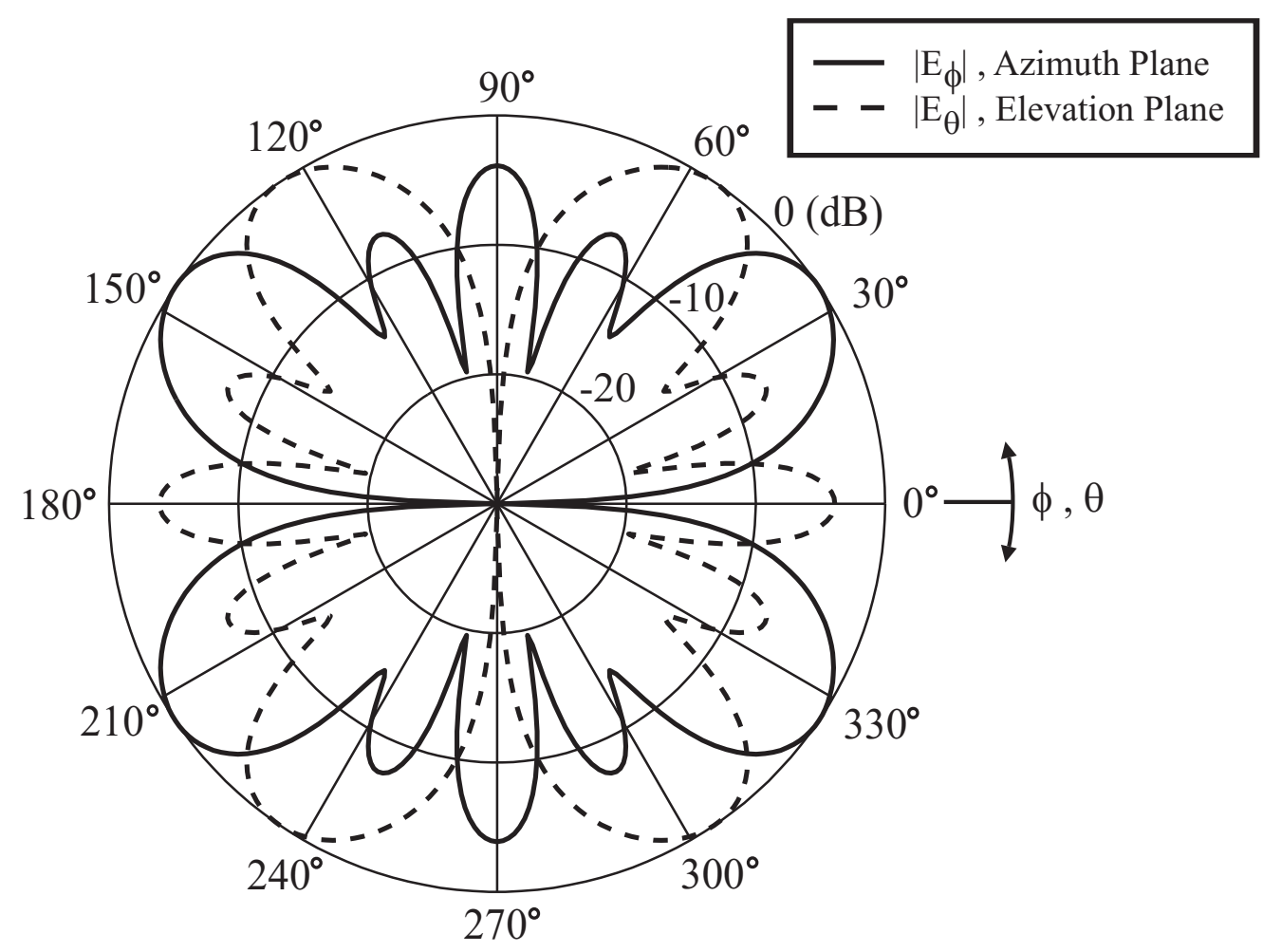

(c)

Figure 85: (Cont'd.) Normalized amplitude patterns for balanced dipole at (a) $\lambda / 2$, (b) $3 \lambda / 2$, and (c) $5 \lambda / 2$ computed using the NEC model in Fig. 76 with $\alpha=0.5$. 


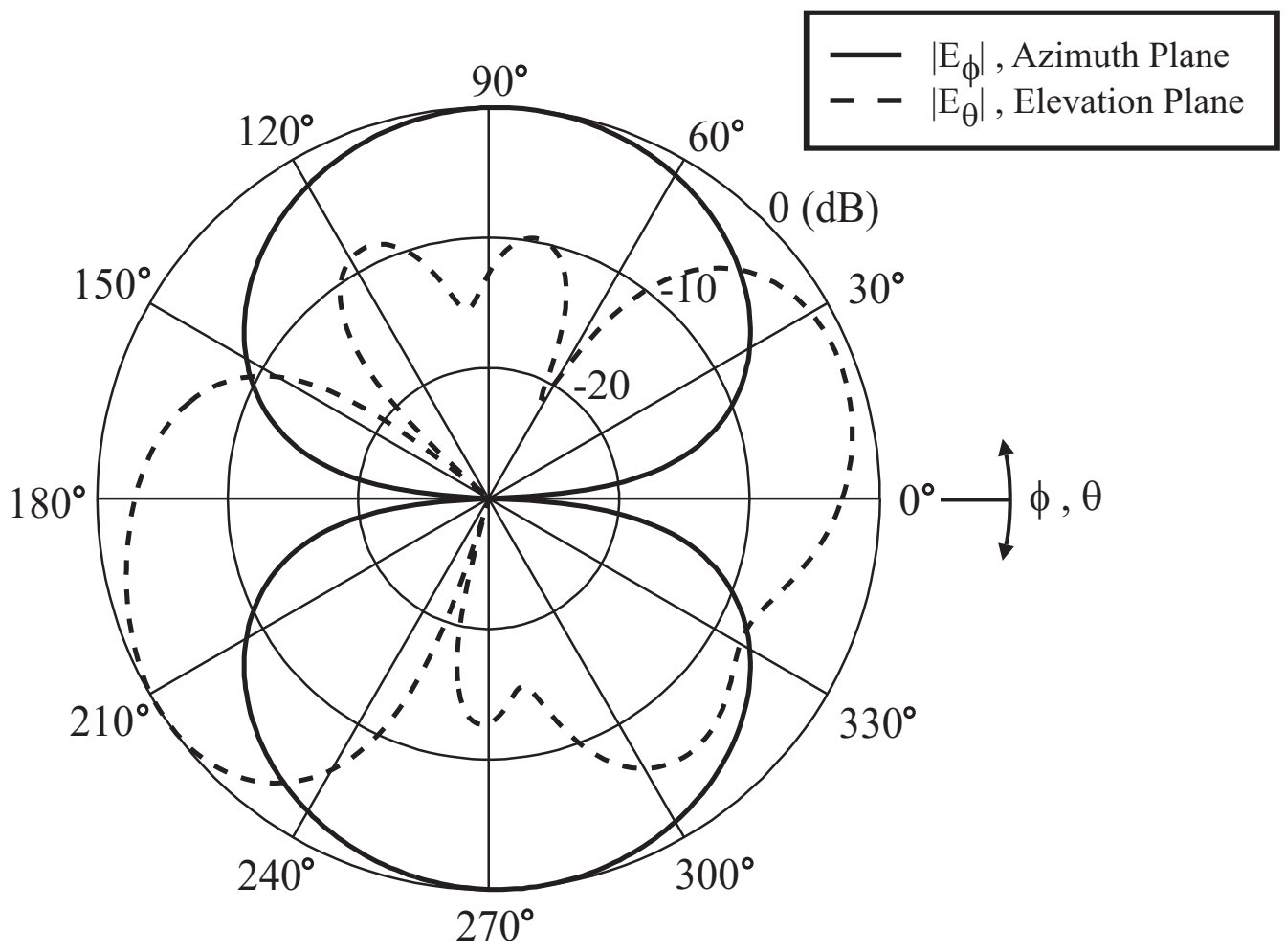

(a)

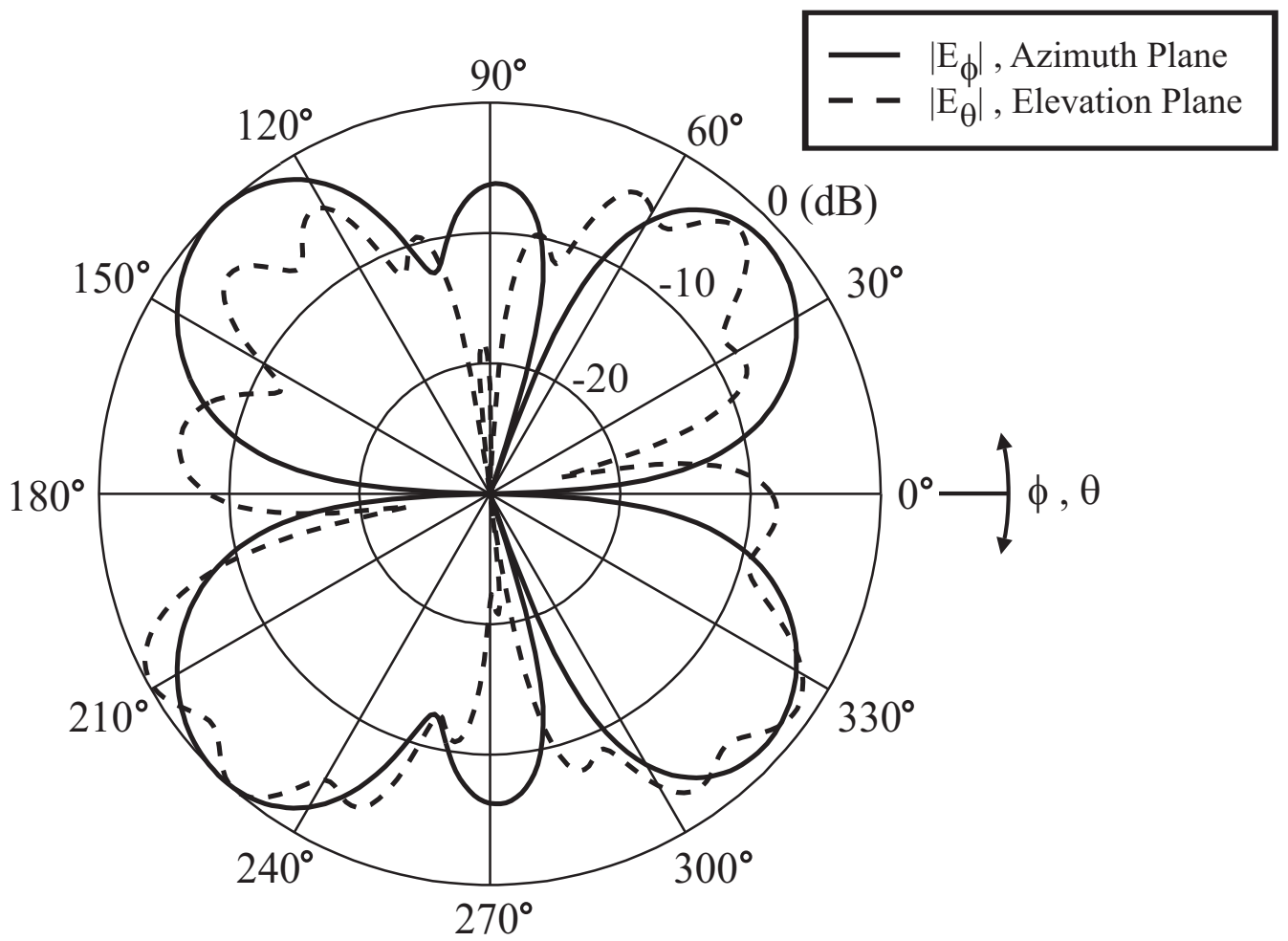

(b)

Figure 86: Normalized amplitude patterns for unbalanced dipole with $5 \lambda / 4$ feedline at (a) $\lambda / 2$, (b) $3 \lambda / 2$, and (c) $5 \lambda / 2$ computed using the NEC model in Fig. 76 with $\alpha=0.1$. 


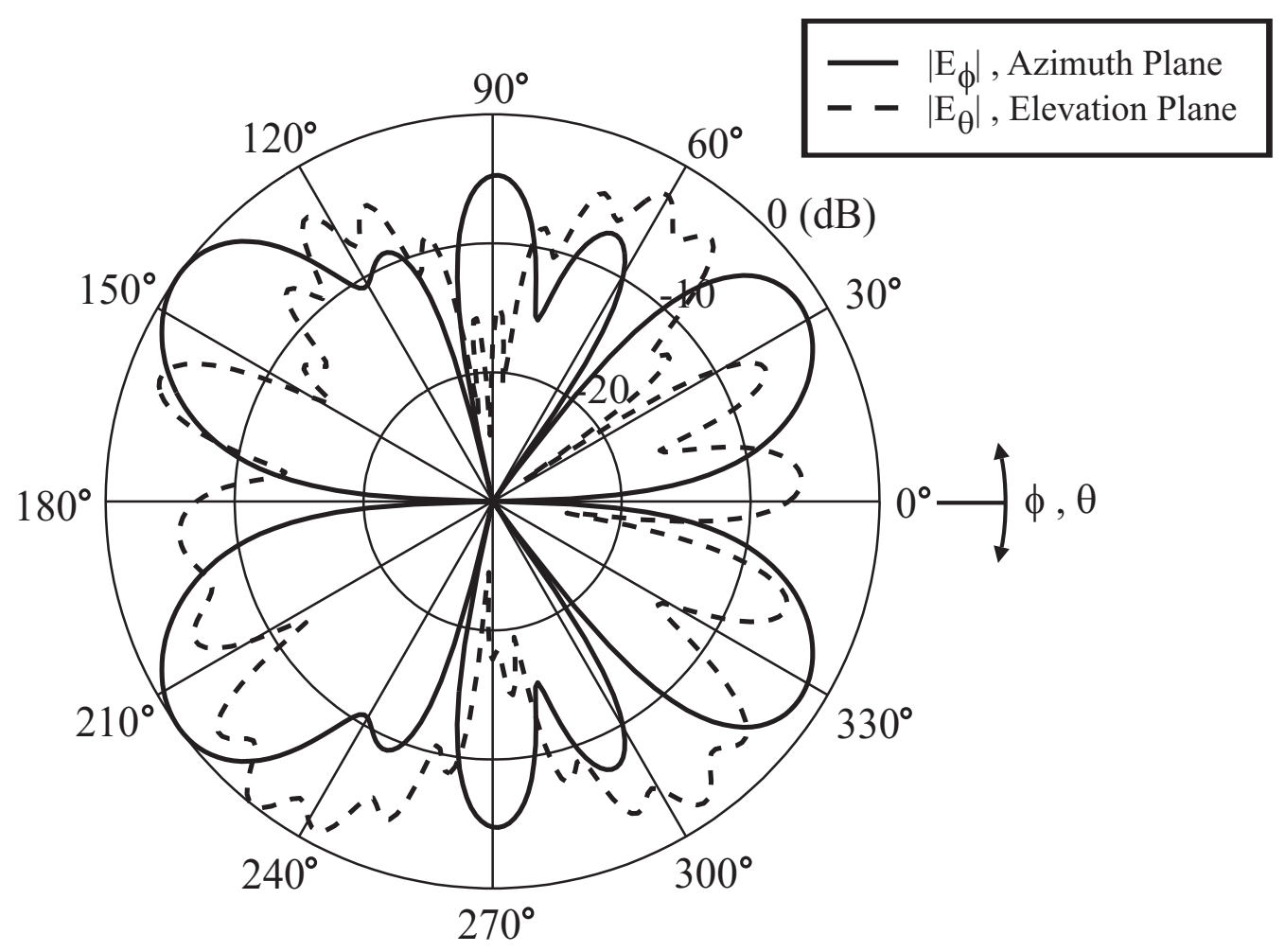

(c)

Figure 86: (Cont'd.) Normalized amplitude patterns for unbalanced dipole with $5 \lambda / 4$ feedline at (a) $\lambda / 2$, (b) $3 \lambda / 2$, and (c) $5 \lambda / 2$ computed using the NEC model in Fig. 76 with $\alpha=0.1$. 


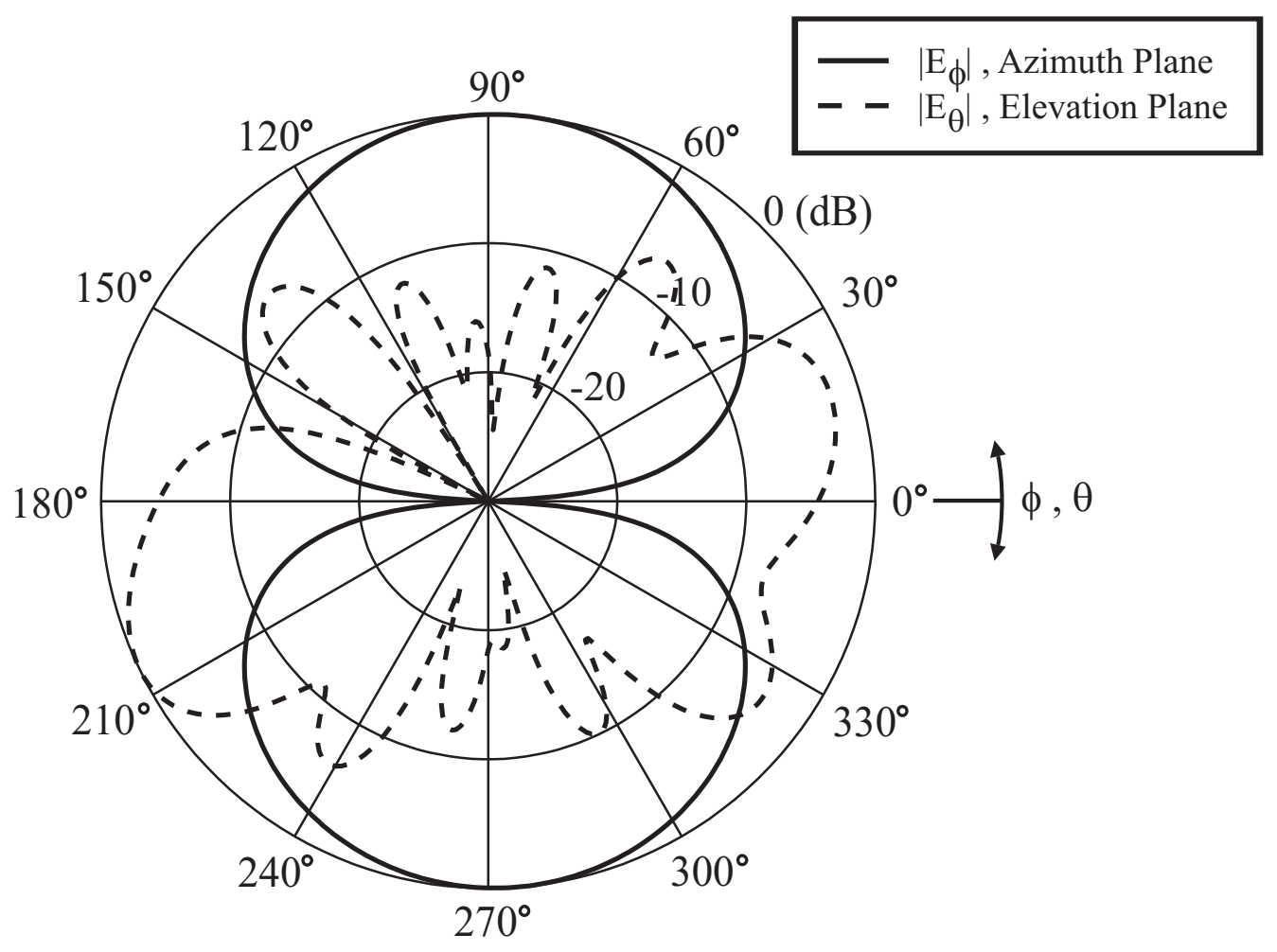

(a)

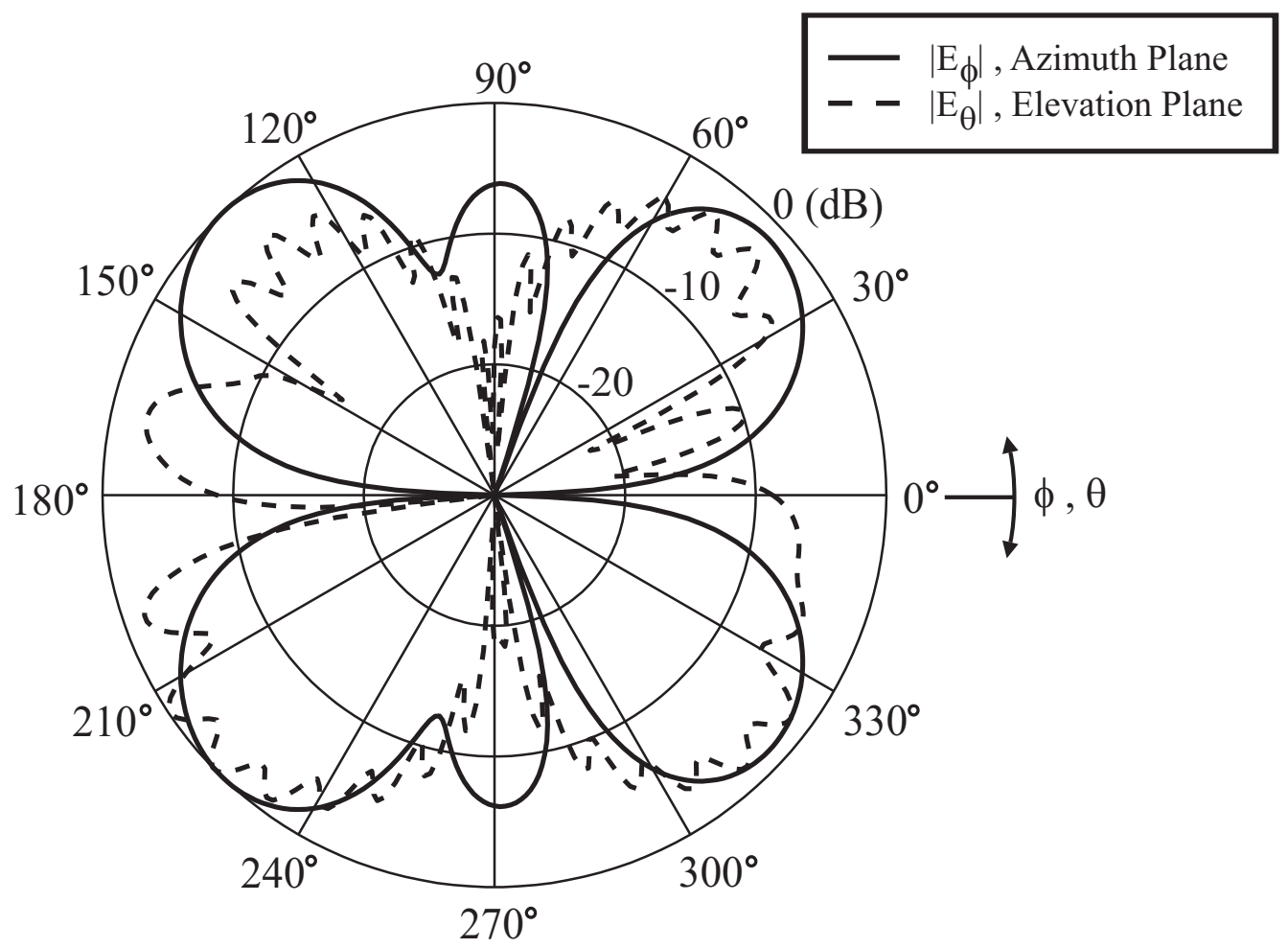

(b)

Figure 87: Normalized amplitude patterns for unbalanced dipole with $11 \lambda / 4$ feedline at (a) $\lambda / 2$, (b) $3 \lambda / 2$, and (c) $5 \lambda / 2$ computed using the NEC model in Fig. 76 with $\alpha=0.1$. 


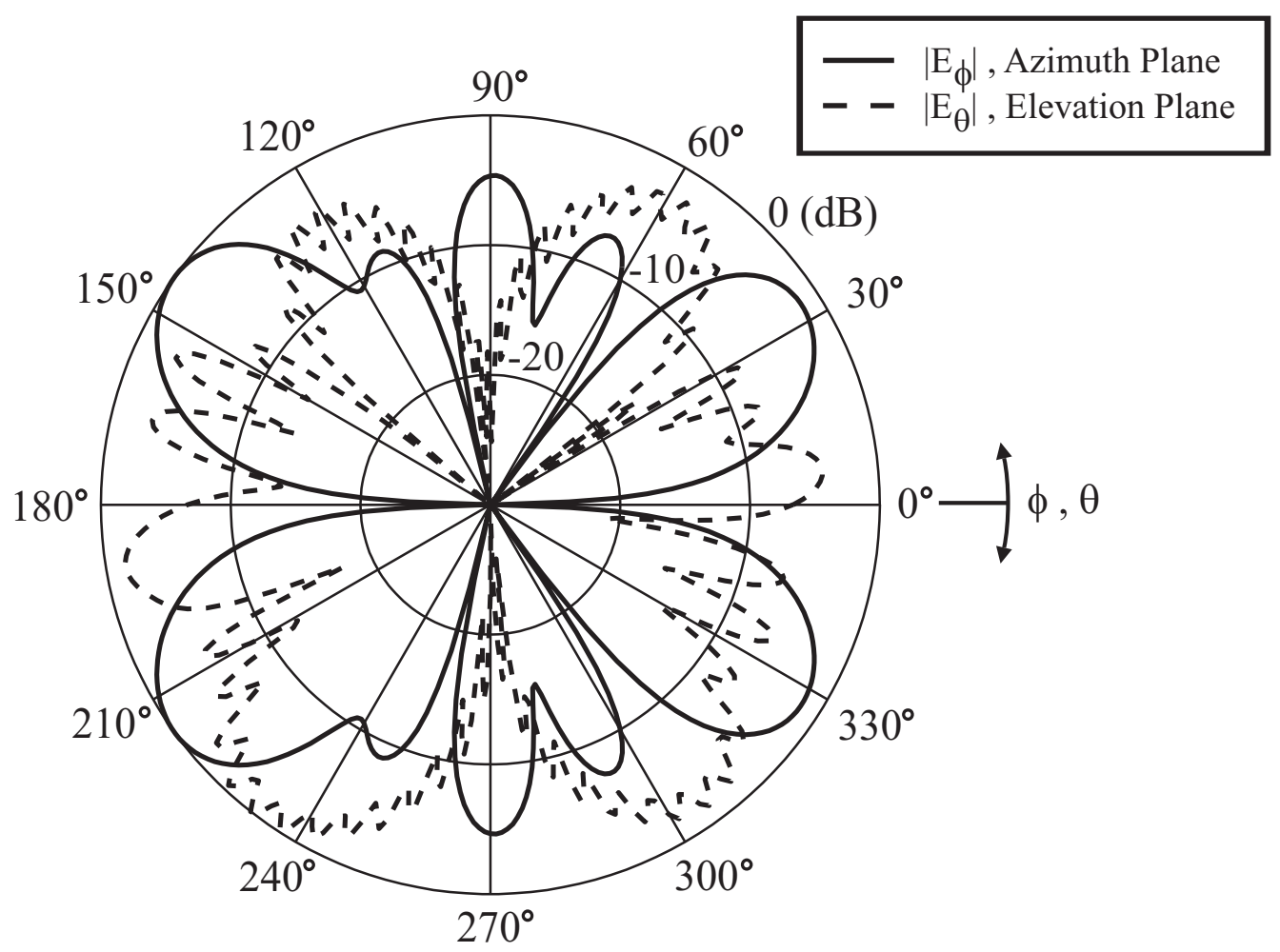

(c)

Figure 87: (Cont'd.) Normalized amplitude patterns for unbalanced dipole with $11 \lambda / 4$ feedline at (a) $\lambda / 2$, (b) $3 \lambda / 2$, and (c) $5 \lambda / 2$ computed using the NEC model in Fig. 76 with $\alpha=0.1$. 


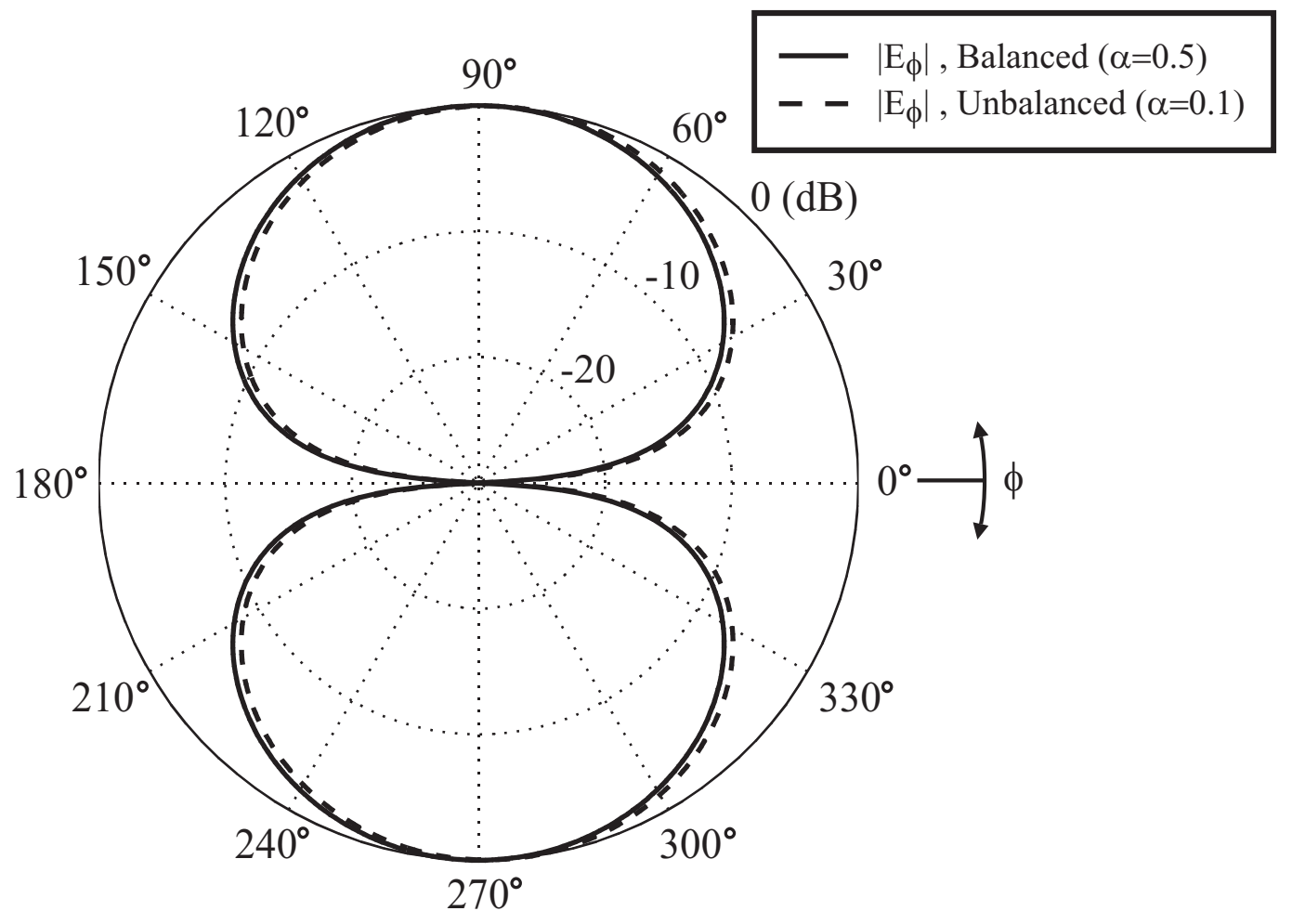

Figure 88: Normalized amplitude pattern computed along azimuth plane, using the NEC model in Fig. 76 with $11 \lambda / 4$ length feedline, for a balanced $(\alpha=0.5)$ dipole and an unbalanced $(\alpha=0.1)$ dipole. 


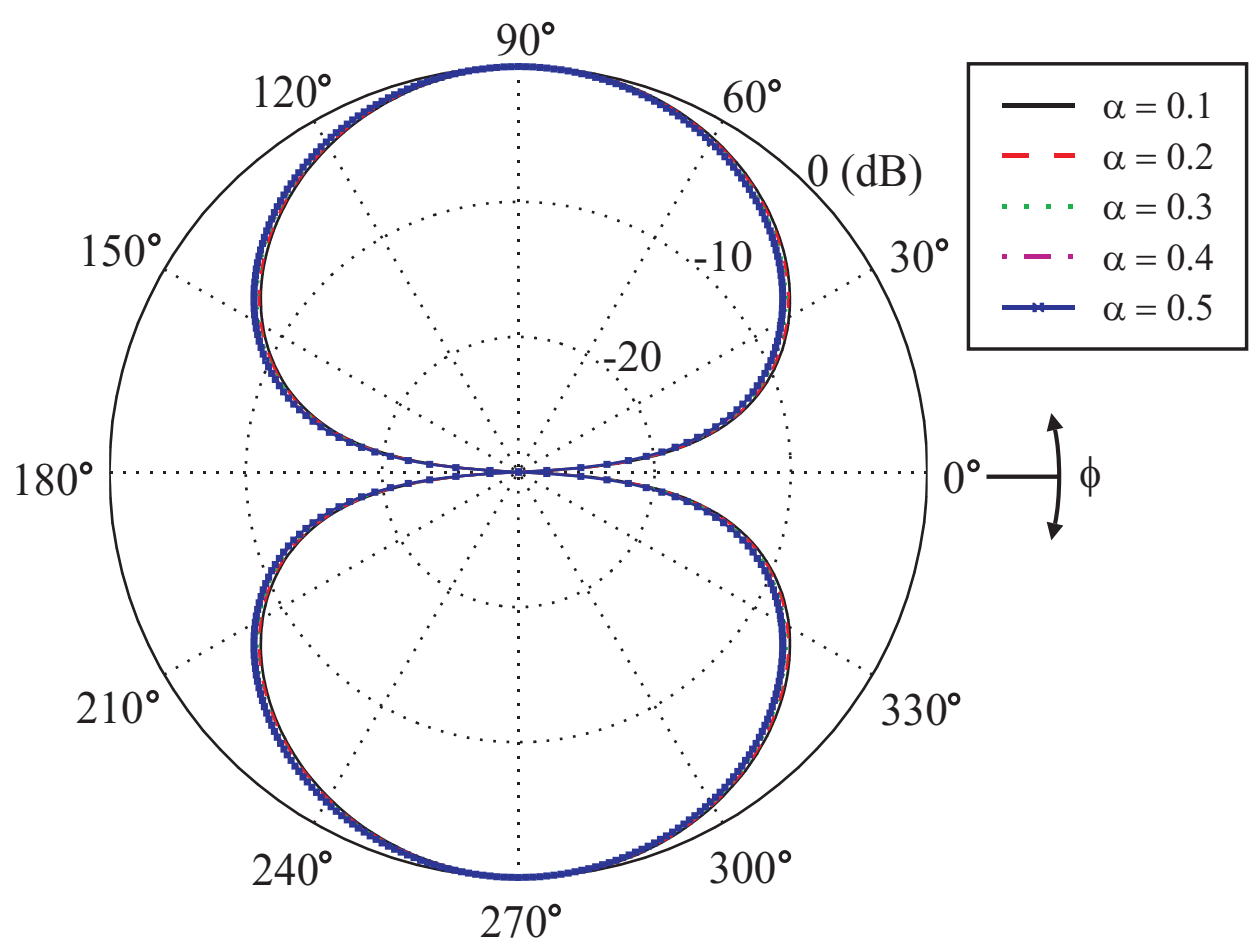

(a)

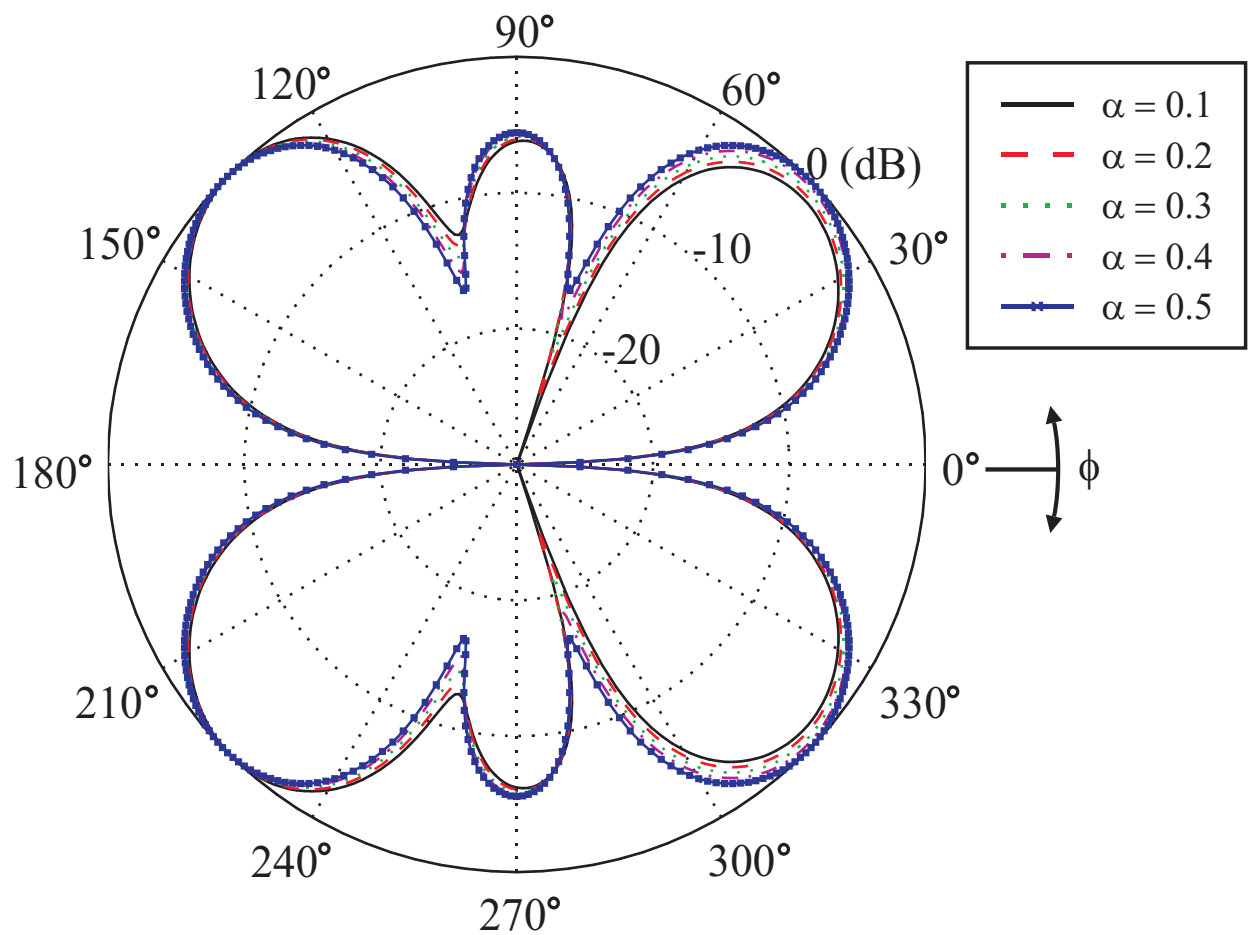

(b)

Figure 89: Normalized amplitude patterns along azimuth plane $\left(\left|E_{\phi}\right|\right)$ for a dipole with $11 \lambda / 4$ feedline at (a) $\lambda / 2$, (b) $3 \lambda / 2$, and (c) $5 \lambda / 2$, computed using the NEC model in Fig. 76 for several values of $\alpha$. 


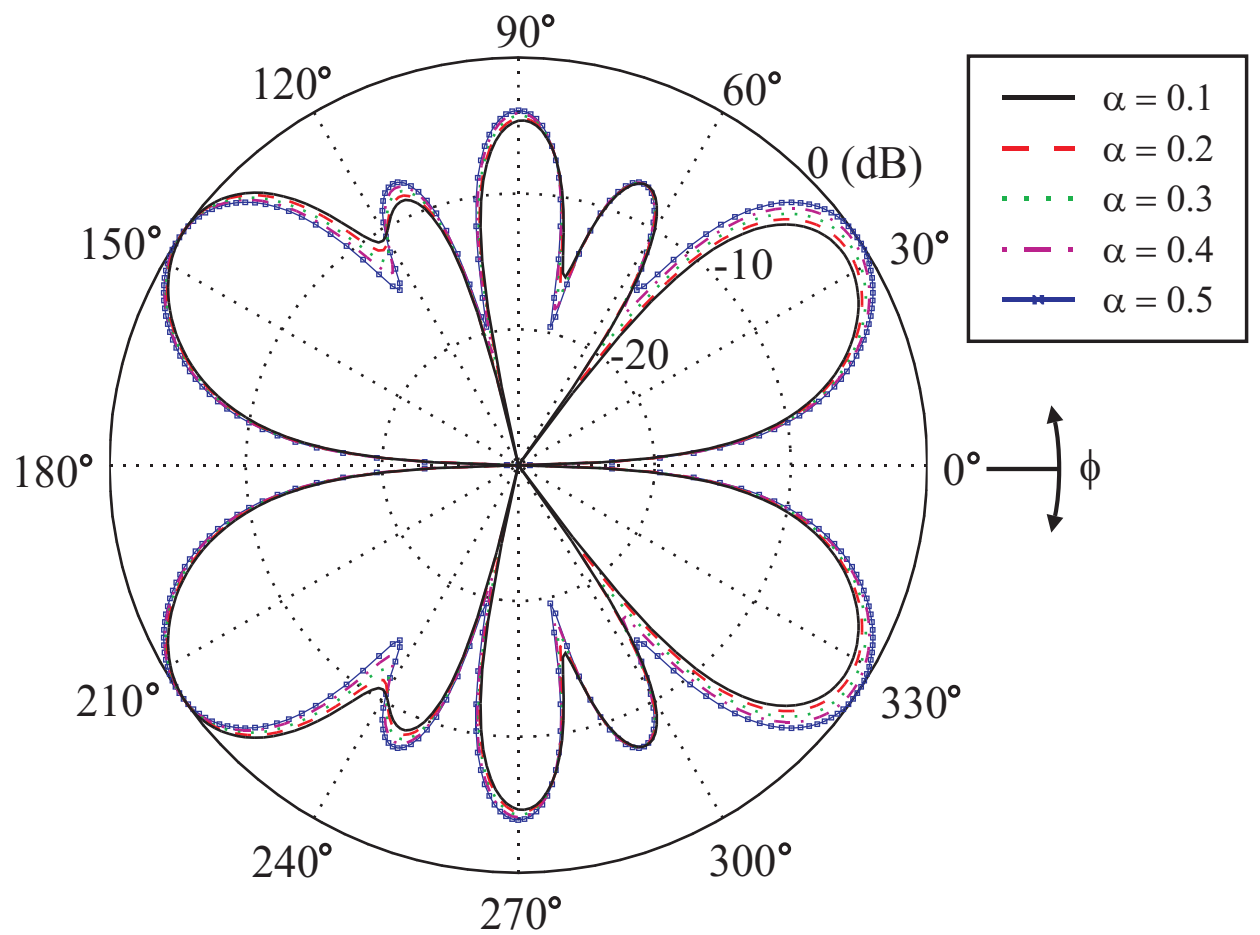

(c)

Figure 89: (Cont'd.) Normalized amplitude patterns along azimuth plane $\left(\left|E_{\phi}\right|\right)$ for a dipole with $11 \lambda / 4$ feedline at (a) $\lambda / 2$, (b) $3 \lambda / 2$, and (c) $5 \lambda / 2$, computed using the NEC model in Fig. 76 for several values of $\alpha$. 


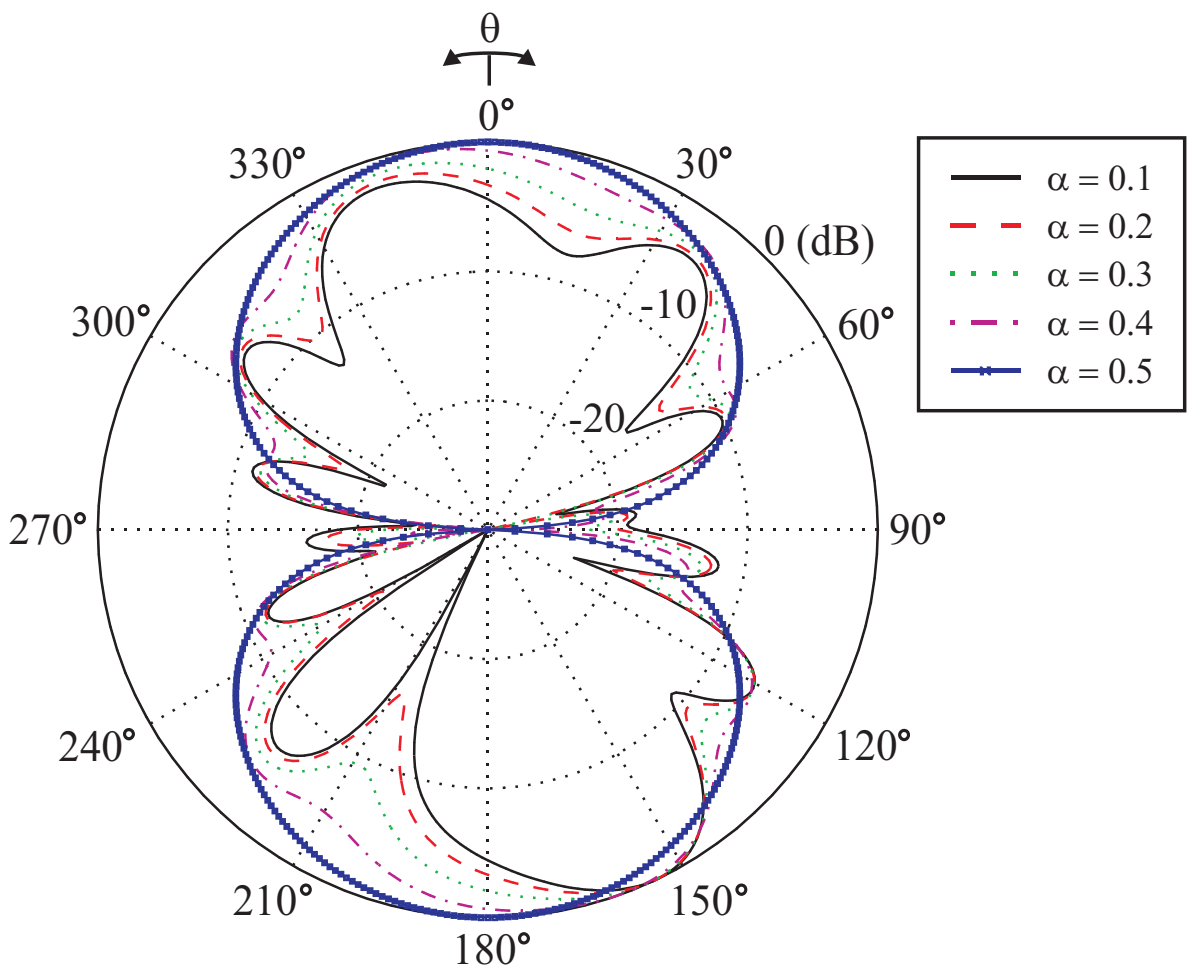

(a)

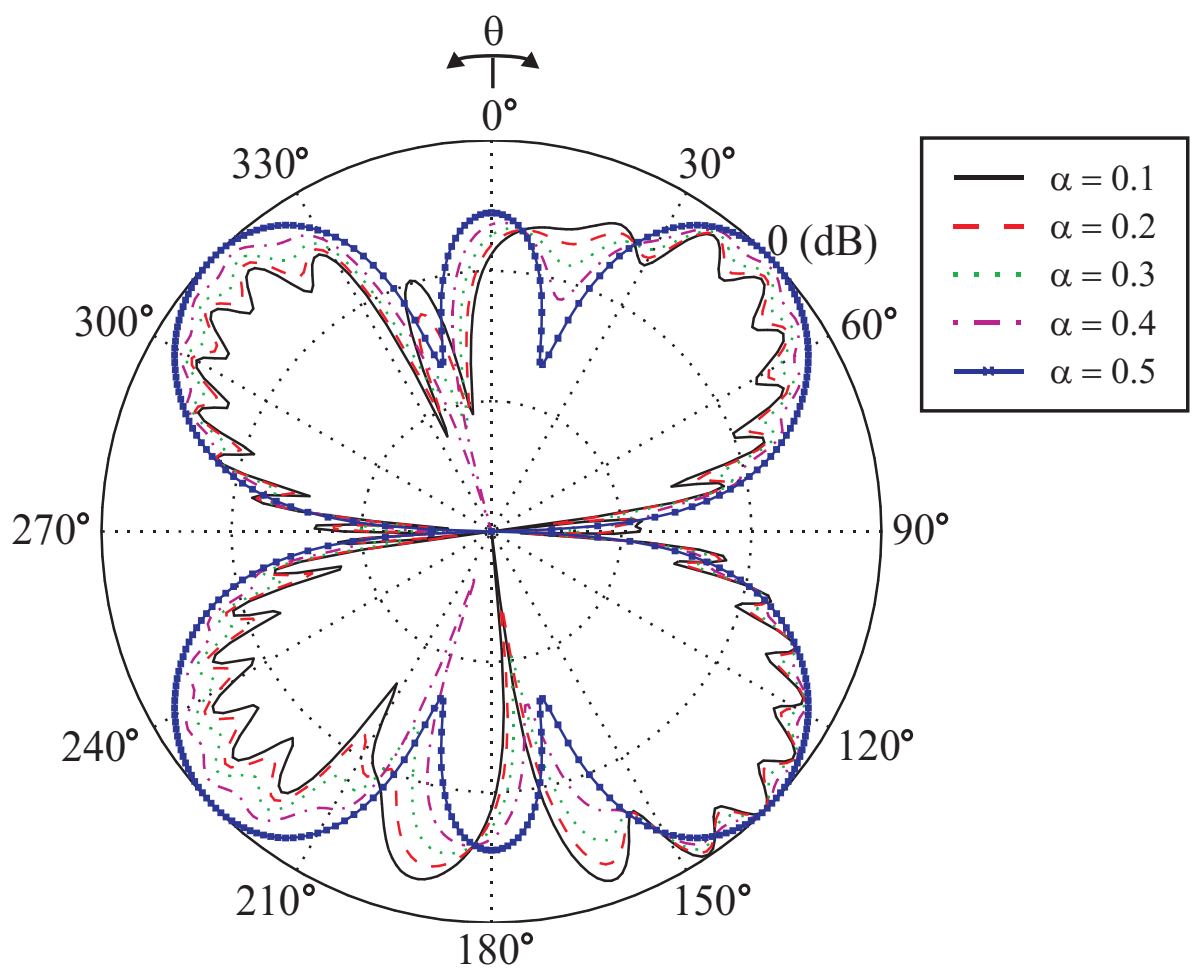

(b)

Figure 90: Normalized amplitude patterns along elevation plane $\left(\left|E_{\theta}\right|\right)$ component) for a dipole with $11 \lambda / 4$ feedline at (a) $\lambda / 2$, (b) $3 \lambda / 2$, and (c) $5 \lambda / 2$ computed using the NEC model in Fig. 76 for several values of $\alpha$. 


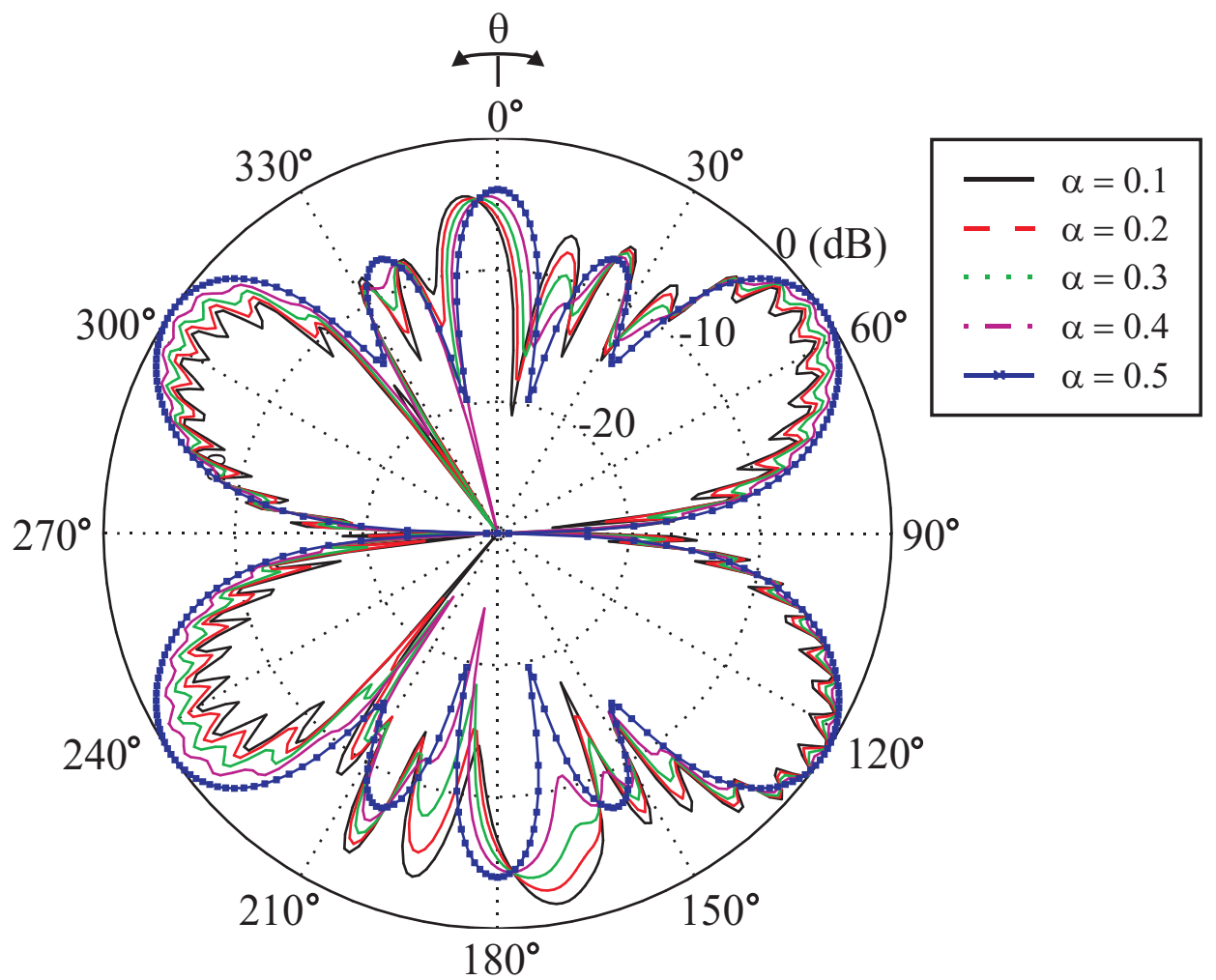

(c)

Figure 90: (Cont'd.) Normalized amplitude patterns along elevation plane $\left(\left|E_{\theta}\right|\right)$ component) for a dipole with $11 \lambda / 4$ feedline at (a) $\lambda / 2$, (b) $3 \lambda / 2$, and (c) $5 \lambda / 2$ computed using the NEC model in Fig. 76 for several values of $\alpha$. 


\subsection{Adding Wu-King Loading}

The previous section investigated the azimuth and elevation plane patterns of an unbalanced dipole with a finite-length feedline. However, in practice, the length of the coaxial feedline can be much longer than that modeled in the previous section. We wish to investigate the effect of an infinitely long feedline on the radiation pattern of an unbalanced dipole. Therefore, to model a feedline of infinite length, a Wu-King loading profile was incorporated into the NEC model in Fig. 76; the modified NEC model is illustrated in Fig. 91. Adding the Wu-King loading is done strictly for numerical purposes to model an infinite feedline. The internal impedance per unit length is given by

$$
z^{i}\left(z^{\prime}\right)=\frac{60 \Psi}{h-\left|z^{\prime}\right|}
$$

where $h$ is the height over which the Wu-King loading is applied, and $\Psi$ is a frequency dependent parameter defined in [32]. Table 2 illustrates values of $\Psi$ computed using Equation 30 (computed for NEC wire segments with radius=0.0001 m) for dipole lengths of $\lambda / 2,3 \lambda / 2$, and $5 \lambda / 2$. The loading profile computed using Equation 30 is illustrated in Fig. 92. The wire segments in the NEC model were loaded with the real and imaginary parts of the impedance profile, illustrated in Fig. 92, using the $L D$ command.

When the arms of the dipole in Fig. 91 are unbalanced, a common-mode current is induced along the feedline. This current undergoes reflections at the antenna feedpoint and at the end of the feedline; a standing wave is generated along the

Table 2: Computed parameters for Wu-King loading along feedline of radius 0.0001 $\mathrm{m}$.

\begin{tabular}{|c|c|c|}
\hline Dipole Length $(\mathrm{m})$ & $h(\mathrm{~m})$ & $\Psi$ \\
\hline$\lambda / 2$ & 0.1 & $8.972-2.984 \mathrm{i}$ \\
\hline $3 \lambda / 2$ & 0.1 & $6.782-3.087 \mathrm{i}$ \\
\hline $5 \lambda / 2$ & 0.1 & $5.757-3.107 \mathrm{i}$ \\
\hline
\end{tabular}


feedline. For an infinitely long feedline, the common-mode current travels along the feedline without any reflections. Practically, the current travels along the feedline and decays exponentially because of conductor and radiation losses. As illustrated in Fig. 91, a Wu-King taper is added towards the end of the feedline to model an infinitely long line. The Wu-King loading causes current to decay as it flows through the loaded segments, hence reducing reflections from the end of the feedline. Small reflections are present along the feedline because of the impedance discontinuity presented by the Wu-King impedance profile. Hence, adding the Wu-King taper only approximates the feedline as being infinite in length. This approximation is sufficient for our purposes. Normalized amplitude patterns, using the NEC model in Fig. 91, are presented next.

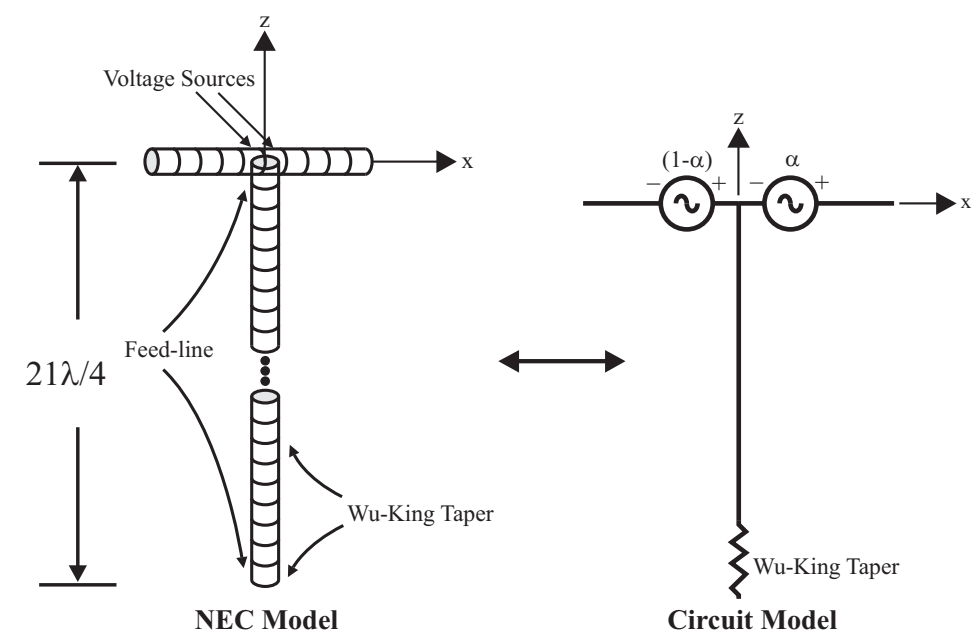

Figure 91: NEC model in Fig. 76 modified to include Wu-King loading along feedline. The Wu-King loading profile is used to approximate an infinite feedline.

A 3-D far field plot for the unbalanced dipole in Fig. 91 ( $\alpha=0.1)$, with Wu-King loading along the feedline, is illustrated in Fig. 93. This pattern is not as distorted as that obtained for an unbalanced dipole with a finite length feedline, illustrated previously in Fig. 83b. This is because the standing waves along the feedline are removed with the Wu-King loading. However, the resulting pattern in Fig. 93 still differs from that of a balanced dipole, illustrated in Fig. 83a. It is seen that the 


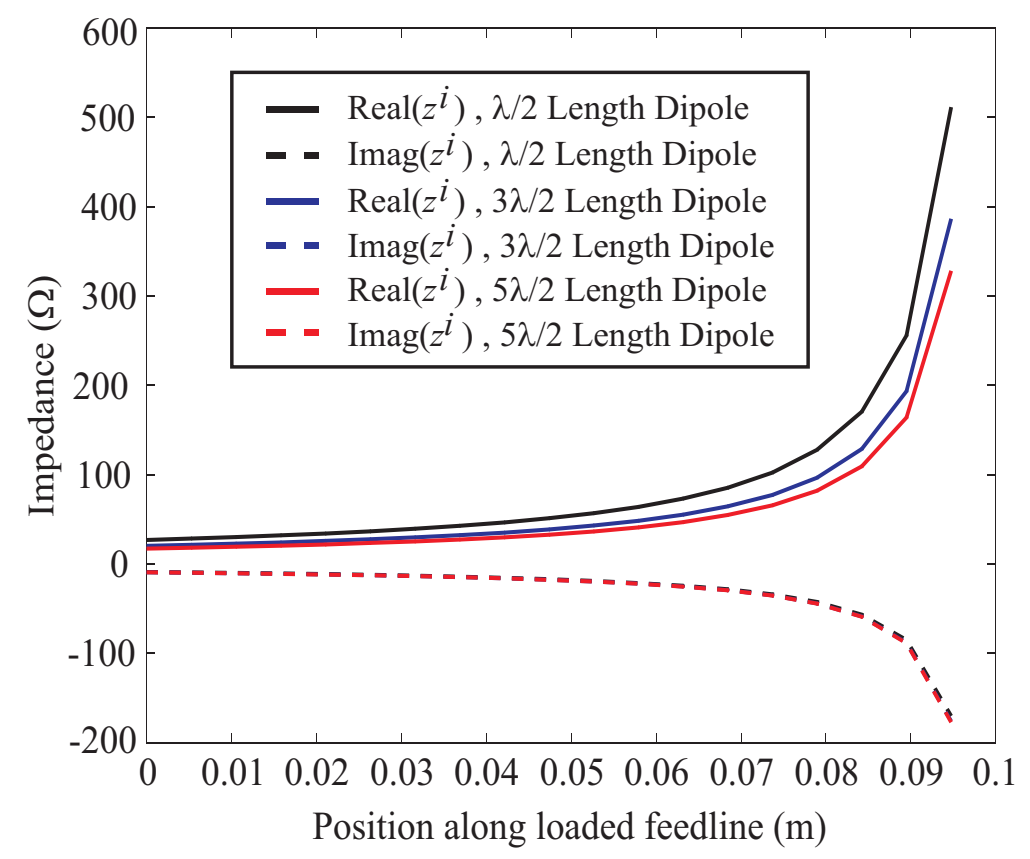

Figure 92: Wu-King loading profile along feedline of NEC model in Fig. 76.

direction of maximum radiation is along the feedline.

Normalized patterns along the azimuth and elevation planes are illustrated in Fig. 94 for the NEC model in Fig. 91 with $\alpha=0.1$ and Wu-King loading along the feedline. It is seen that loading the feedline has less effect on the resulting amplitude patterns along the azimuth plane than along the elevation plane. When compared to the elevation plane patterns of an unbalanced dipole with a finite length feedline in Figs. 87 and 86, the patterns along the elevation plane for the unbalanced dipole with feedline loading exhibit improved symmetry.

A dipole, along with the outer conductor of the coaxial line, was modeled in Section 3.1. In Section 3.2, amplitude patterns along the azimuth and elevation planes were generated for balanced and unbalanced dipoles with a finite length feedline. However in practice, the coaxial feedline could be much longer than the wavelength. Therefore, to approximate an infinite feedline, the feedline in Fig. 76 was extended to be $21 \lambda / 4$ and loaded with a Wu-King impedance profile, as illustrated in Fig. 91. The resulting patterns, presented in this section, illustrate that varying the length of the feedline has 

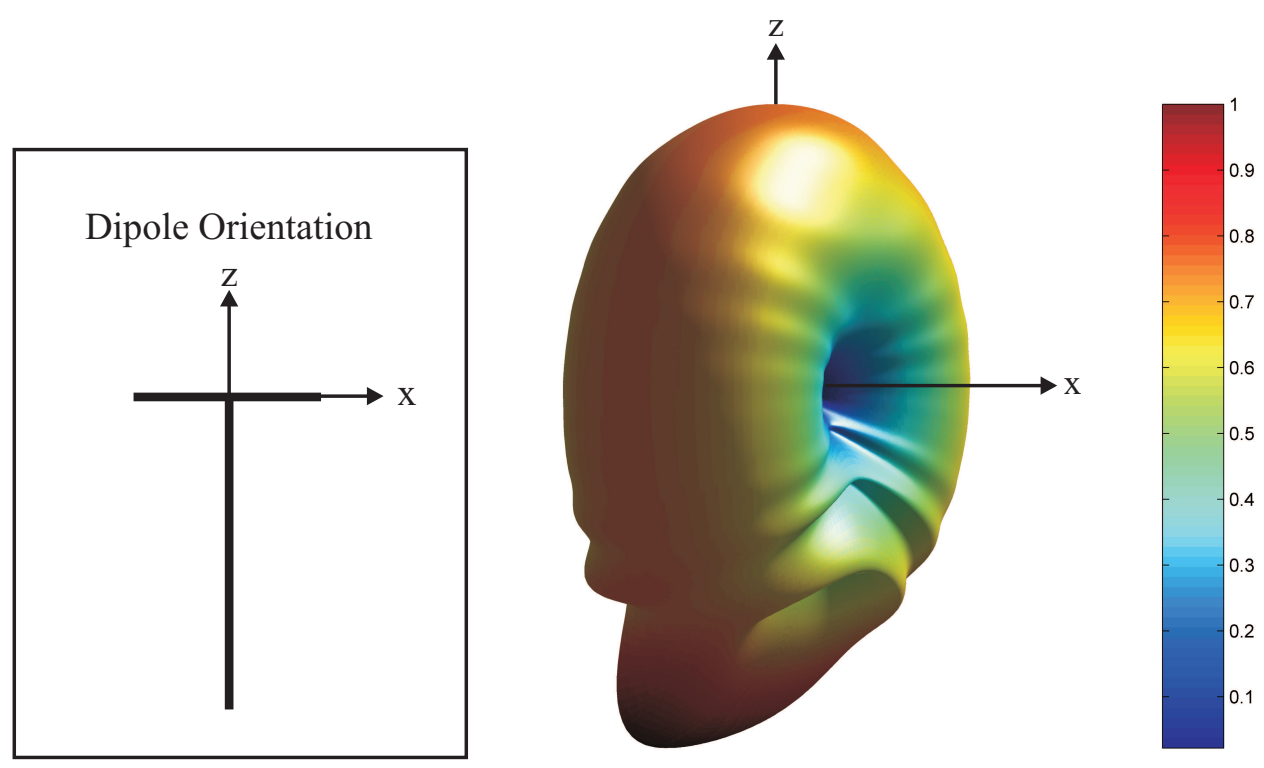

Figure 93: 3-D normalized far field pattern of unbalanced dipole generated using NEC model in Fig. 91 with $\alpha=0.1$ and Wu-King loading along feedline of length $21 \lambda / 4$.

little effect on the amplitude patterns along the azimuth plane. However, the infinite feedline does affect the pattern along the elevation plane by improving the pattern symmetry in this plane for an unbalanced dipole. While symmetry is improved, the patterns still illustrate an unbalanced feed. Thus, the length of the feedline clearly affects the pattern along the elevation plane. 


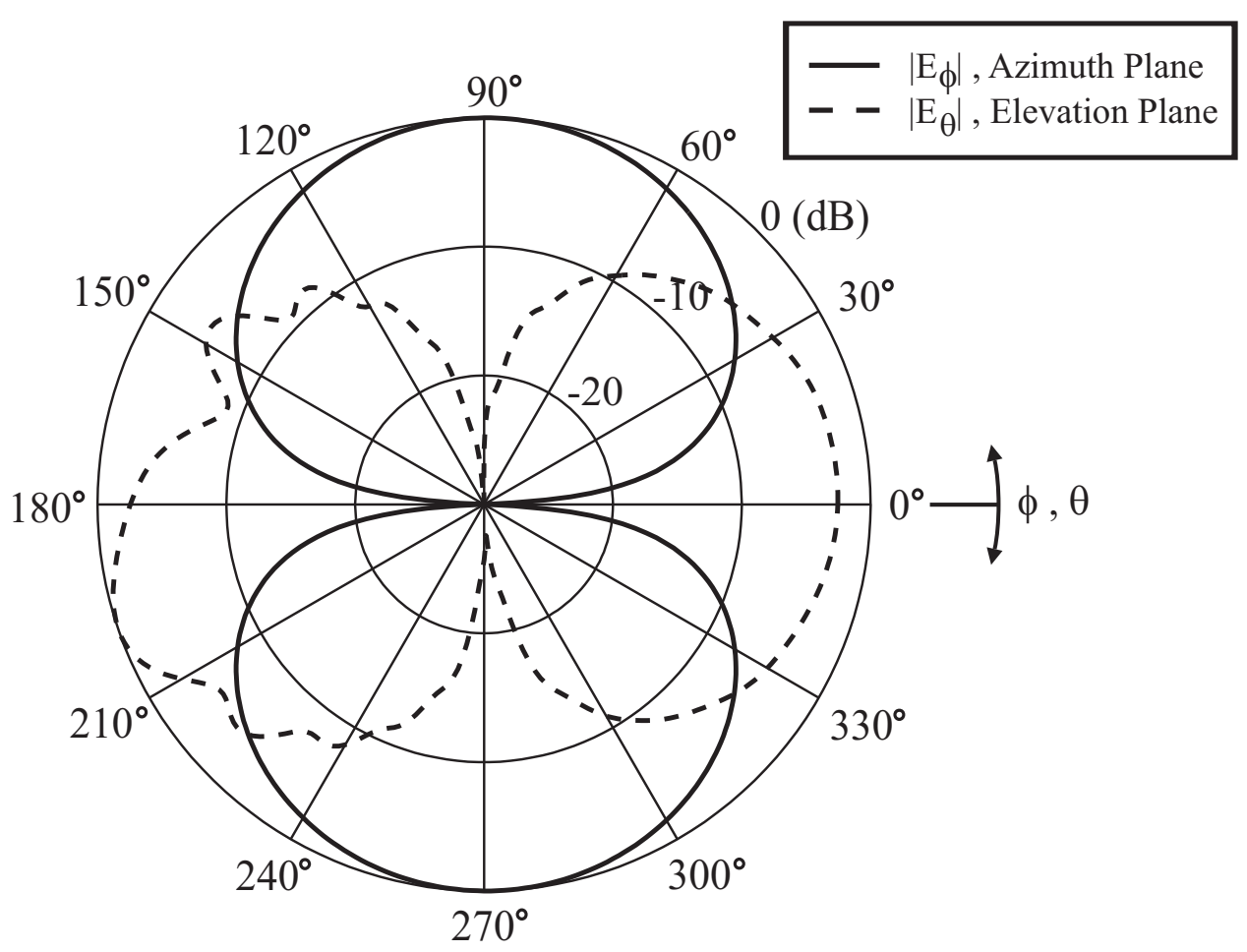

(a)

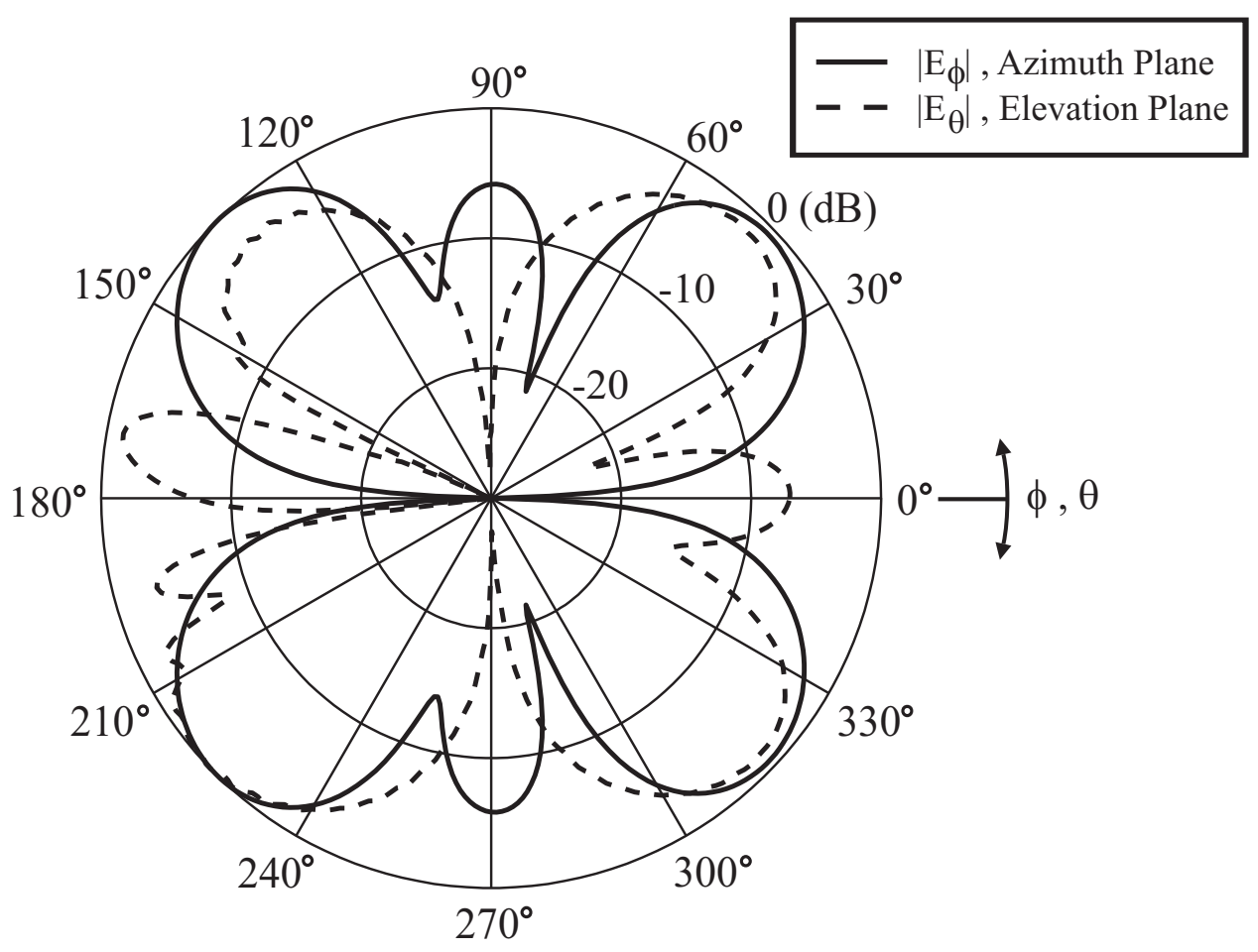

(b)

Figure 94: Normalized amplitude patterns for unbalanced dipole with Wu-King loading along feedline of length $21 \lambda / 4$, at (a) $\lambda / 2$, (b) $3 \lambda / 2$, and (c) $5 \lambda / 2$ computed using NEC model in Fig. 91. 


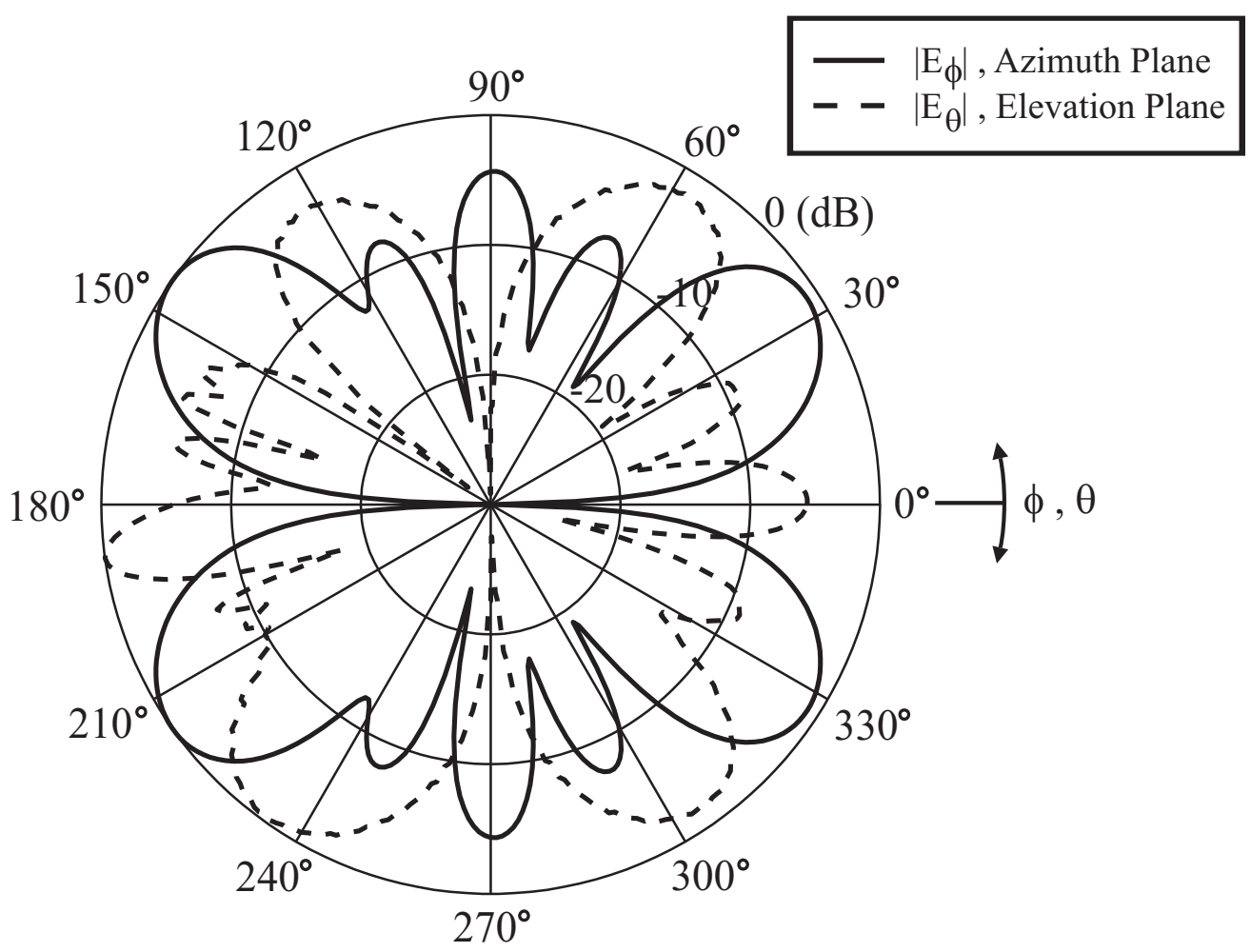

(c)

Figure 94: (Cont'd.) Normalized amplitude patterns for unbalanced dipole with Wu-King loading along feedline of length $21 \lambda / 4$ at (a) $\lambda / 2$, (b) $3 \lambda / 2$, and (c) $5 \lambda / 2$ computed using NEC model in Fig. 91. 


\subsection{Fields of Dipole and Travelling-Wave Antenna}

When the arms of a dipole are unbalanced, a common-mode current is induced along the feedline. This current causes the feedline to radiate. In Section 3.2, patterns of an unbalanced dipole with a finite length feedline were presented. In Section 3.3, patterns of an unbalanced dipole with Wu-King loading along the feedline were presented. The Wu-King loading approximated the feedline as being infinitely long. Radiation from an unbalanced dipole with a long feedline (length of feedline $\gg \lambda[33]$ ), can be modeled as the superposition of the fields radiated by a balanced dipole and the fields radiated by a travelling wave antenna (current along the long feedline causes it to behave as a travelling wave antenna). In this section, analytical expressions are derived for the far-field electric field radiated by a balanced dipole oriented along the $\mathrm{x}$-axis and a travelling wave antenna oriented along the $\mathrm{z}$-axis. The superposition of these fields, computed analytically, is compared with the pattern obtained using NEC for an unbalanced dipole with Wu-King loading along the feedline.

Figure 34 illustrates the far-field amplitude pattern of a travelling-wave antenna, computed by modeling just the feedline (21 $\lambda / 4$ length feedline in Fig. 91) with WuKing loading in NEC. The far-field amplitude pattern of a balanced dipole was illustrated previously in Fig. 83. The superposition of these amplitude patterns should approximate the pattern of an unbalanced dipole with a long feedline, illustrated in Fig. 93.

The electric field of a dipole (in the far-field), with arms oriented along the x-axis, is given by

$$
\begin{aligned}
& E_{\theta}=\frac{-\mu_{o} I_{o} e^{-j k r} \omega}{4 \pi r}\left[\frac{e^{\frac{j k l}{2} \cos \phi \sin \theta}-e^{\frac{j k l}{2}}}{j k(\cos \phi \sin \theta-1)}+\frac{e^{-\frac{j k l}{2}}-e^{\frac{j k l}{2} \cos \phi \sin \theta}}{j k(-1-\cos \phi \sin \theta)}\right] \cos \phi \cos \theta \\
& E_{\phi}=\frac{\mu_{o} I_{o} e^{-j k r} \omega}{4 \pi r}\left[\frac{e^{\frac{j k l}{2} \cos \phi \sin \theta}-e^{\frac{j k l}{2}}}{j k(\cos \phi \sin \theta-1)}+\frac{e^{-\frac{j k l}{2}}-e^{\frac{j k l}{2} \cos \phi \sin \theta}}{j k(-1-\cos \phi \sin \theta)}\right] \sin \phi,
\end{aligned}
$$



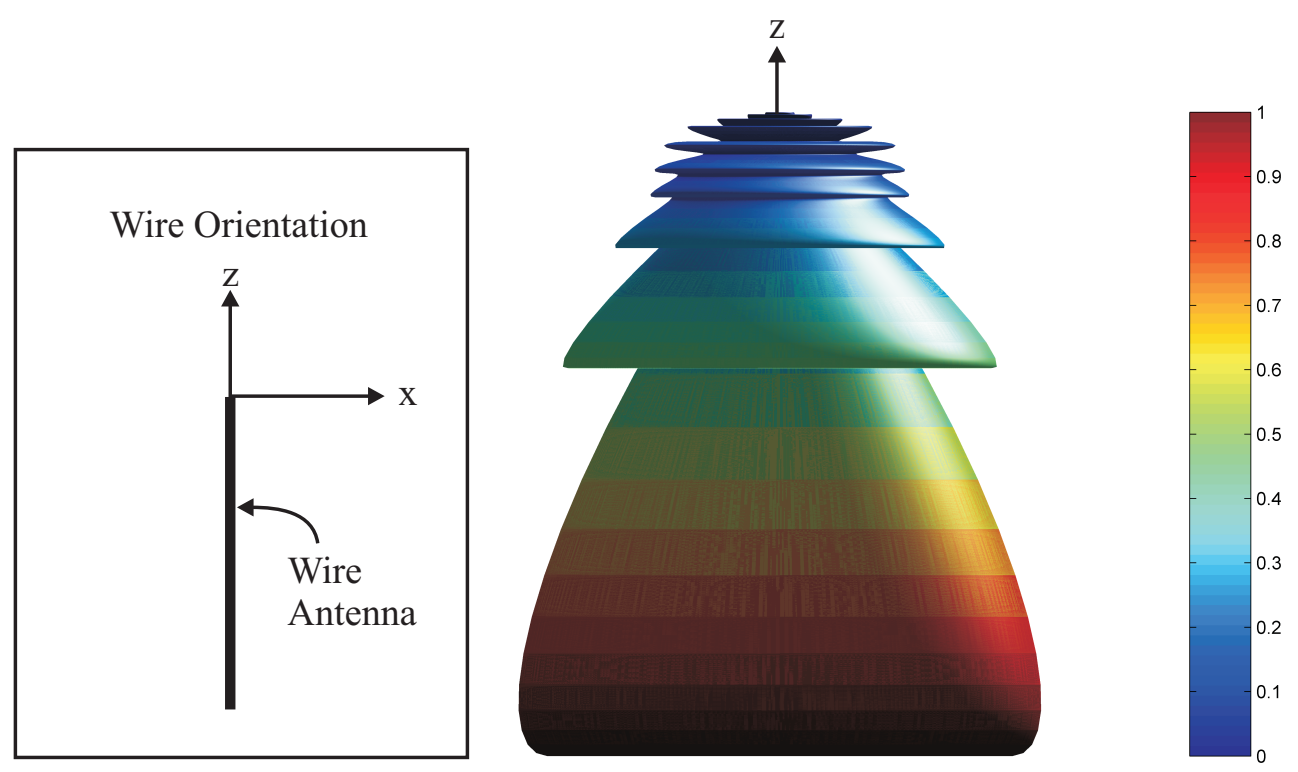

Figure 95: 3-D normalized far field pattern of travelling-wave wire antenna, modeled in NEC with Wu-King loading along feedline of length $21 \lambda / 4$.

where the current along the dipole arms is assumed to have the form

$$
I_{x}=I_{o} \sin \left[k\left(\frac{l}{2}-\left|x^{\prime}\right|\right)\right] .
$$

The electric field (in the far-field) of a travelling-wave wire antenna is given by

$$
E_{\theta}=\frac{\mu_{o} I_{o f} e^{-j k r} \omega}{4 \pi r}\left[\frac{e^{-j k l(1+\cos \theta)}-1}{k(1+\cos \theta)}\right] \sin \theta
$$

where the current along the wire (models the feedline of an unbalanced dipole) is assumed to have the form

$$
I_{z}=I_{o f} e^{j k z}
$$

Figure 96 illustrates the theoretical far-field pattern obtained via the superposition of fields given by Equations 31-35. It is seen that the pattern in Fig. 96 agrees well with that obtained for an unbalanced dipole with Wu-King loading along the feedline, in Fig. 93. Ripples are observed in the pattern in Fig. 96; these ripples result from the finite length feedline used in the analytical model. In this section, it was shown that the far field amplitude pattern of an unbalanced dipole with a long feedline $(\gg$ 
$\lambda[33])$ can be approximated by superposition of the fields radiated by a balanced dipole and a travelling wave wire antenna.
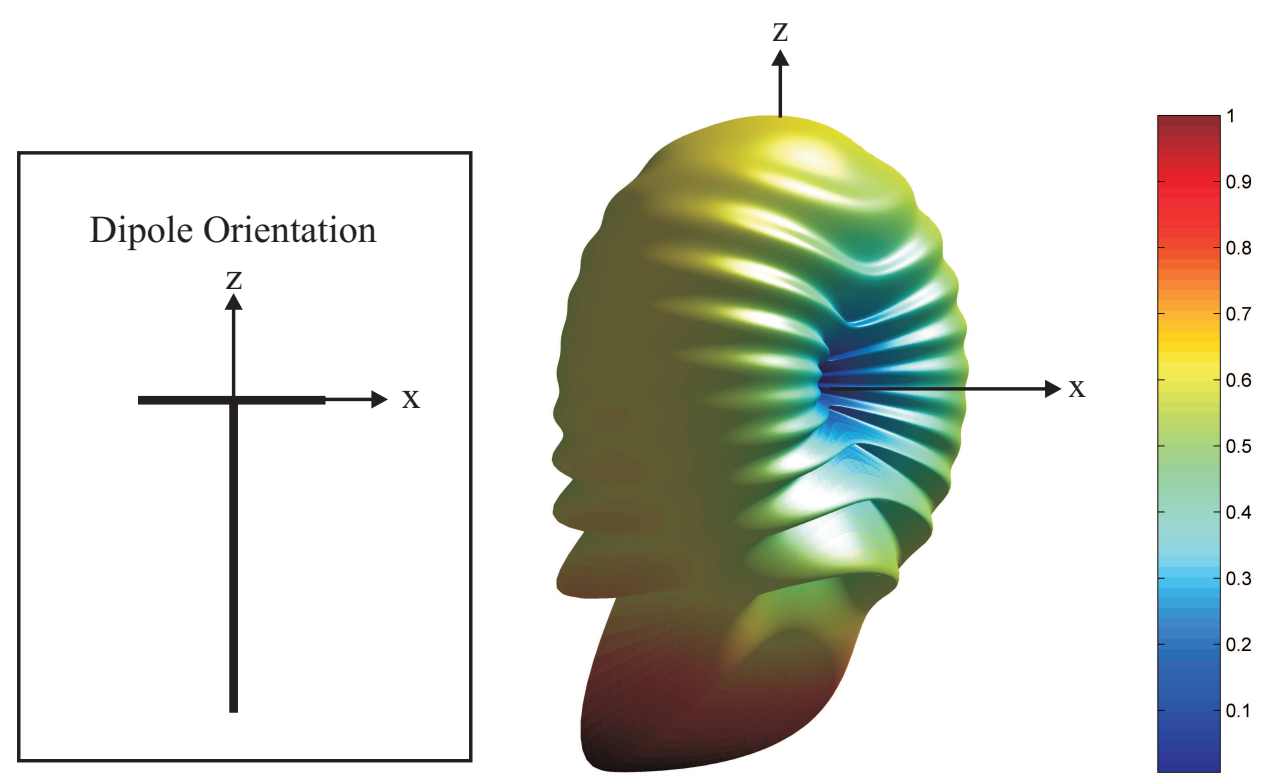

Figure 96: 3-D normalized far-field pattern illustrating superposition of fields from balanced dipole and travelling-wave wire antenna, computed using Equations 31-35. 


\subsection{Time-Domain Analysis}

\subsubsection{Time Gating Reflected Pulses}

In this section, the effects of an unbalanced dipole feed are studied in the time domain. Investigating an unbalanced dipole in the time domain provides us with better physical insight into the effects of an unbalanced feed. The NEC model in Fig. 76 was expanded to include a second dipole, as illustrated in Fig. 97. This expanded NEC model was used to conduct time-domain analysis of balanced and unbalanced dipoles fed with the double-y balun designed in Section 2.7. The balun transitions from a $50 \Omega$ CPW section to a $200 \Omega$ CPS line (dipole is fed with the CPS line). Since the dipoles in Fig. 97 are assumed to be fed with the balun, an impedance mismatch exists between the dipoles and the CPS section of the double-y balun. This impedance mismatch must be accounted for when obtaining the transfer function between the dipoles.

As illustrated in Fig. 97, voltage sources were placed on the arms of the transmitting (TX) dipole; the two center segments of the receiving (RX) dipole were each loaded with $100 \Omega$ to model the impedance mismatch created by the double-y balun feeding the RX dipole. To model a balanced dipole, the voltage sources were modeled as done in Fig. 76, with $\alpha=0.5$. However, to model the unbalanced case, only one of the sources was used, with a value of $1 \mathrm{~V}(\alpha=0)$. Only one source was used because the input impedance of the antenna computed by NEC was used to calculate the transfer function of the dipoles. The input impedance computed by NEC is clearly defined only when the two sources are balanced or when there is only one source.

The two dipole model in Fig. 97 is represented in Fig. 98 as a two port network to better illustrate the derivation of the transfer function of the dipoles. In the equations that follow, frequency domain is implied with the use of $(f)$, and time domain is implied with the use of $(t)$. The frequency-domain transfer function between the two 
dipoles in Fig. 97 is given by

$$
V_{\text {dipole }}(f)=\frac{V_{L}(f)}{V_{\text {in }}(f)},
$$

where $V_{L}(f)$ is the voltage across the $200 \Omega$ resistively loaded segments of the RX dipole; $V_{i n}(f)$ is defined shortly. The voltage across the $200 \Omega$ segments, $V_{L}(f)$, is given by

$$
V_{L}(f)=I_{L}(f) R_{L}
$$

where $I_{L}$ is the load current computed by NEC across the $200 \Omega$ segments, $R_{L}$. $V_{\text {source }}(f)$ is defined as the $1 \mathrm{~V}$ source voltage applied to the TX dipole arms. Since it is assumed that both, the TX and RX dipoles are fed with the double-y balun, the effect of the $200 \Omega$ characteristic impedance of the CPS section must also be included for the TX dipole. To do this, the source voltage can be related to the input voltage, $V_{\text {in }}(f)$ in Equation 36, by

$$
V_{\text {source }}(f)=(1+\Gamma(f)) V_{\text {in }}(f),
$$

where $\Gamma(f)$ is given by

$$
\Gamma(f)=\frac{Z_{\text {in }}(f)-Z_{o}}{Z_{\text {in }}(f)+Z_{o}} .
$$

In Equation $39, Z_{\text {in }}(f)$ is the input impedance of the TX dipole computed by NEC, and $Z_{o}$ is the $200 \Omega$ characteristic impedance of the double-y balun's CPS section. Hence, with the above relations, the frequency-domain transfer function between the two dipoles, taking into account the mismatch created by the characteristic impedance of the double-y balun's CPS section, is given by

$$
T_{\text {dipole }}(f)=\frac{I_{L}(f) R_{L}}{V_{\text {source }}(f)}(1+\Gamma(f)) .
$$

Having derived the frequency-domain transfer function in Equation 40 between the two dipoles in Fig. 97, we now wish to obtain the time-domain voltage at the RX 
dipole. Figure 99a illustrates a time-domain plot of a differentiated Gaussian pulse, given by

$$
V_{i n c}(t)=-V_{o}\left(\frac{t}{\tau_{p}}\right) \exp \left\{-\left[\left(\frac{t}{\tau_{p}}\right)^{2}-1\right] / 2\right\},
$$

where $V_{o}$ is a constant, and $\tau_{p}$ is the pulse width given by

$$
\tau_{p}=\frac{1}{2 \pi f_{p}}
$$

The frequency spectrum of the differentiated Gaussian pulse in Fig. 99a is illustrated in Fig. 99b. It is seen that the spectral peak occurs at $f_{p}$. The time-domain voltage received by the RX dipole in Fig. 97 is given by

$$
V_{\text {dipole }}(t)=i f f t\left[\left\{\frac{I_{L}(f) R_{L}}{V_{\text {source }}(f)}(1+\Gamma(f))\right\} \cdot V_{\text {inc }}(f)\right]
$$

where ifft is the inverse fast-Fourier transform.

Figure 100 compares the time-domain voltage at the receiving dipole (RX), as defined by Equation 43, for balanced and unbalanced $5 \mathrm{~cm}$ dipoles (length of dipole arms is $5 \mathrm{~cm}$ ). The transmitting dipole (TX) is excited with a $1 \mathrm{~V}$-peak differentiated Gaussian pulse having a spectral peak at 3 GHz. In Fig. 100, additional reflections are observed for the case where the dipole arms are unbalanced. These reflections are caused by the common-mode current induced along the feedline of the unbalanced dipole. In the time domain, a pulse travels down the feedline of the unbalanced dipole, reflects at the end of the finite-length feedline, and travels up the feedline again towards the dipole arms, where part of the pulse is transmitted to the arms of the dipole (corresponds to the reflections observed in Fig. 100) and part of the pulse reflects back down the feedline. For the balanced dipole, no such reflections are observed because there is no current induced along the feedline.

In Sections 3.2 and 3.3, amplitude patterns were generated for balanced and unbalanced dipoles using the NEC models in Fig. 76 and 91, respectively. These patterns were generated to illustrate the effects of an unbalanced dipole feed. In this section, 


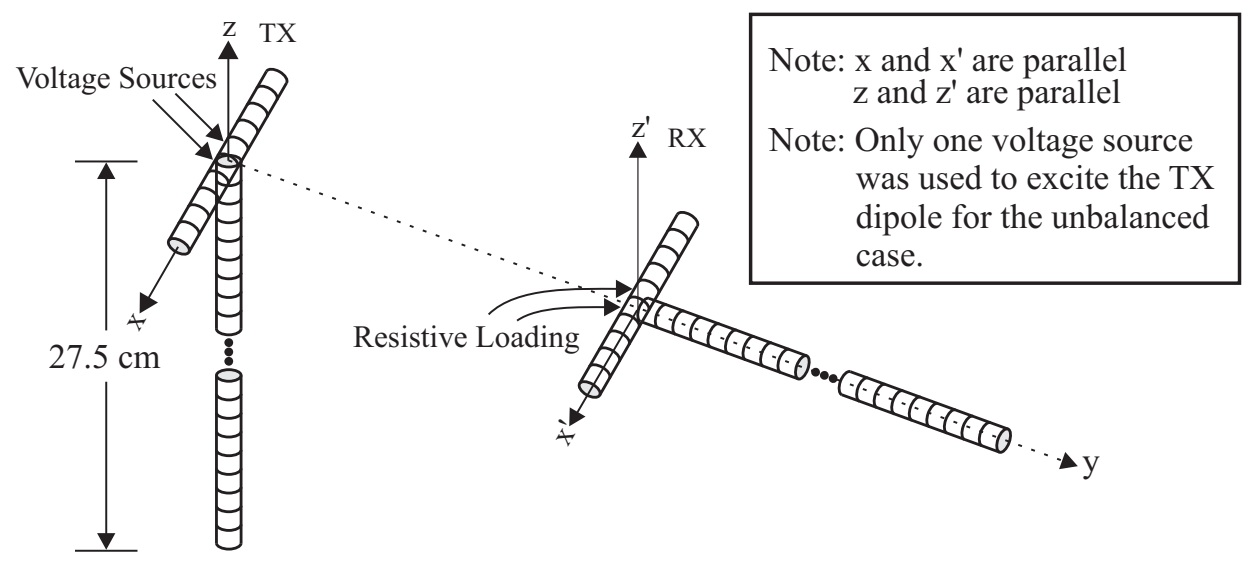

Figure 97: NEC model used to study balanced/unbalanced dipoles fed with a double-y balun in the time domain.

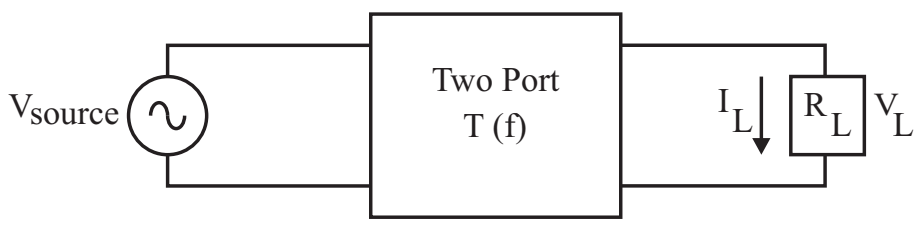

Figure 98: Representation of NEC model in Fig. 97 as a two port network.

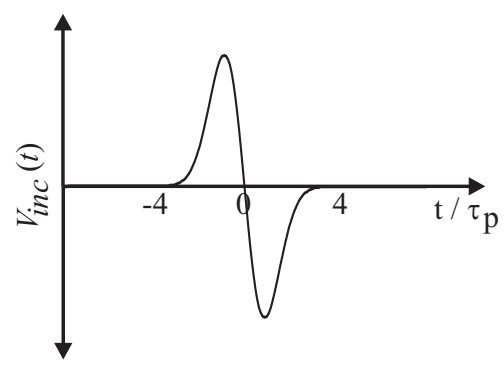

(a)

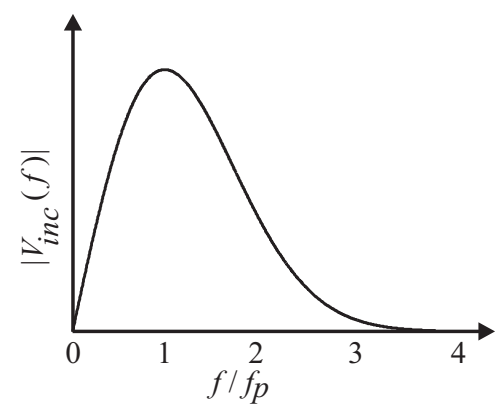

(b)

Figure 99: Illustration of (a) a differentiated Gaussian pulse in the time domain with pulse width $\tau_{p}$, and (b) its frequency spectrum showing spectral peak at $f_{p}$. 


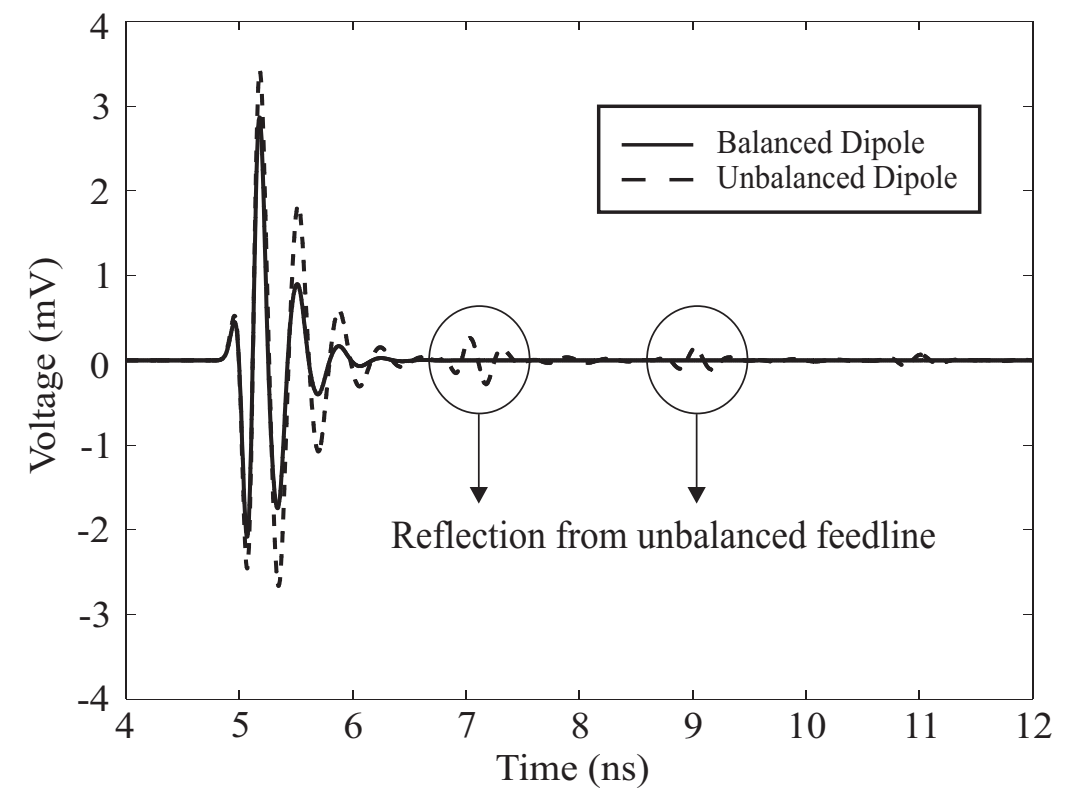

Figure 100: Time-domain voltage received by RX dipole in Fig. 97 illustrating feedline reflections from unbalanced TX dipole. The TX dipole was excited with a differentiated Gaussian pulse with spectral peak at $3 \mathrm{GHz}$.

an unbalanced dipole feed was analyzed in the time domain using the NEC model in Fig. 97. Additional reflections were observed in the received time-domain voltage waveform when the transmitting dipole was unbalanced. Next, the effect of these additional reflections on the normalized amplitude patterns is investigated. The effect of time-gating the reflections from the feedline of an unbalanced dipole on the resulting amplitude patterns at $\lambda / 2$ and $3 \lambda / 2$ is investigated.

To investigate the effect of the feedline reflections on the resulting antenna pattern, the unbalanced TX dipole in Fig. 97 was rotated $360^{\circ}$ along the azimuth direction in increments of $5^{\circ}$, as illustrated in Fig. 101. The received time-domain voltage pattern by the RX dipole at each angle is illustrated in Fig. 102, where secondary reflections are observed due to the unbalanced feed. Figure 103 illustrates an expanded view of the time-domain pattern in Fig. 102 at $90^{\circ}$. With no time gating applied, all reflections from the feedline are allowed to exist. With an $8.5 \mathrm{~ns}$ time gate, only the first reflection from the feedline is allowed in the time window; the later reflections are zeroed out. The 6.5 ns time gate removes all reflections from the feedline. 
The time-domain plot at each angle was transformed into the frequency domain without time gating any reflections; the resulting frequency domain patterns at $\lambda / 2$ and $3 \lambda / 2$ are illustrated in Figs. 104a and 104b, for the three time gating configurations applied in Fig. 103. Without any time gating, the azimuth patterns in Fig. 104 should and do match the patterns generated in NEC in Fig. 87. The nulls are not as deep because the patterns in Fig. 104 were generated in five degree increments instead of two. There is little difference in the patterns in Fig. 104 for the case where no time gating was applied and the case where time gating started at $8.5 \mathrm{~ns}$. Similarly, time gating all feedline reflections (time gating at $6.5 \mathrm{~ns}$ ) has little effect on the resulting patterns along the azimuth plane, as illustrated in Fig. 104. Hence, time gating the feedline reflections has little impact on the amplitude pattern along the azimuth plane. To understand why time gating the feedline reflections has little impact on the azimuth pattern, we observe that the TX dipole is oriented such that the RX dipole only receives the radiated fields from the dipole arms. Therefore, only the component of the feedline reflection that is transferred to the dipole arms affects the pattern. This component is very small, as observed from Figs. 103 and 104.

Next, the effect of time gating the feedline reflections on the amplitude pattern along the elevation plane is investigated. The NEC model in Fig. 101 was modified as illustrated in Fig. 105. The resulting time-domain amplitude pattern is illustrated in Fig. 106. It is interesting to note that reflections appear at all angles. As was done previously with the time-domain patterns along the azimuth plane, time gating was applied at $6.5 \mathrm{~ns}$ and $8.5 \mathrm{~ns}$, as illustrated in Fig. 107. However, it is seen that time gating the feedline reflections does alter the resulting pattern significantly along the elevation plane, as illustrated in Fig. 108. To understand why time gating the feedline reflections significantly affects the pattern along the elevation plane, we observe that the TX dipole in Fig. 105 is oriented such that the RX dipole receives the radiated fields from the dipole arms as well as the feedline. Therefore, when the 
reflections from the feedline are time gated out as is Fig. 107, the radiated fields from the feedline are altered, thus affecting the patterns along the elevation plane in in Fig. 108. It is seen that the time-domain pattern in Fig. 103 is identical to that in Fig. 107. This occurs because in both cases the TX dipole is at an angle such that the RX dipole only receives the radiated fields from the dipole arms.

In this section, time-domain analysis was conducted for an unbalanced dipole using NEC. Reflections from the feedline were observed in the time domain for an unbalanced dipole. The effects of time gating these reflections on the resulting amplitude patterns were investigated. Resulting amplitude patterns showed that time gating feedline reflections had little effect on the resulting pattern along the azimuth plane. However, time gating the reflections did alter the resulting pattern along the elevation plane. This is in agreement with the analysis conducted in the previous section, where the elevation pattern varied with feedline length. Removing the feedline reflections in the time domain is equivalent to modeling an infinite feedline.

When conducting pattern measurements, reflections from nearby objects can clutter the measured data. These reflections can be removed from the measured data in the time domain. However, as illustrated by the results in this section, reflections are also observed in the time domain from the feedline of an unbalanced dipole. Timegating the first reflection from the feedline affects the patterns along the elevation plane significantly; this reflection should not be time-gated out. Thus, results in this section suggest that sufficient time window should be allowed in the measured data to capture the first reflection from the feedline, before time-gating out the clutter. 


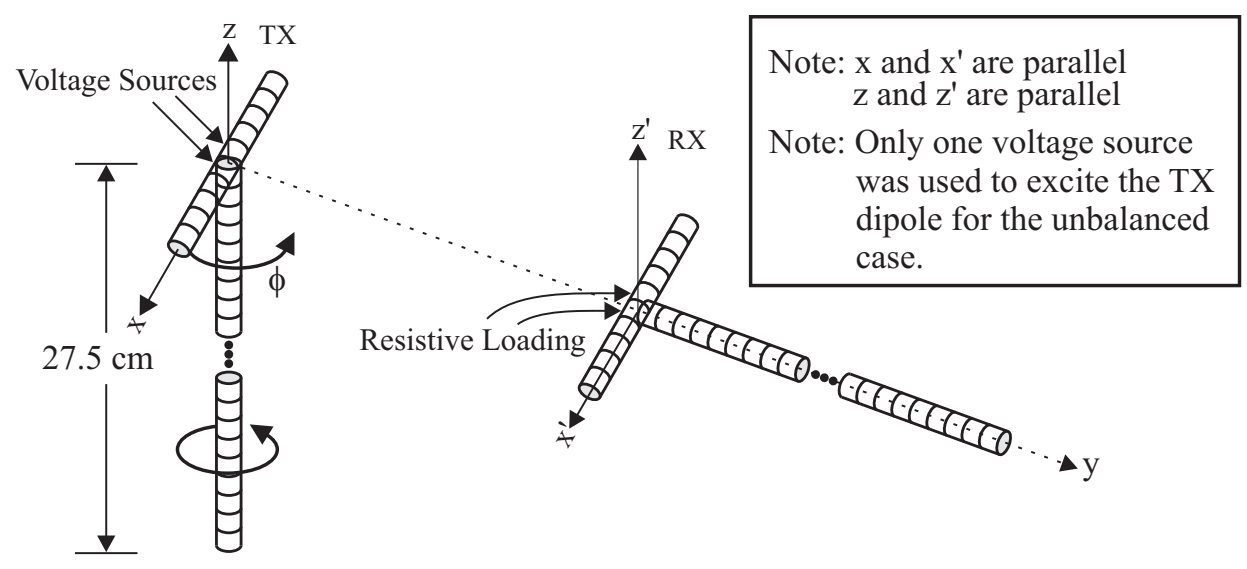

Figure 101: NEC model used to study the effect of time gating secondary reflections caused by unbalanced dipole feed on the frequency domain amplitude pattern along the azimuth plane. 


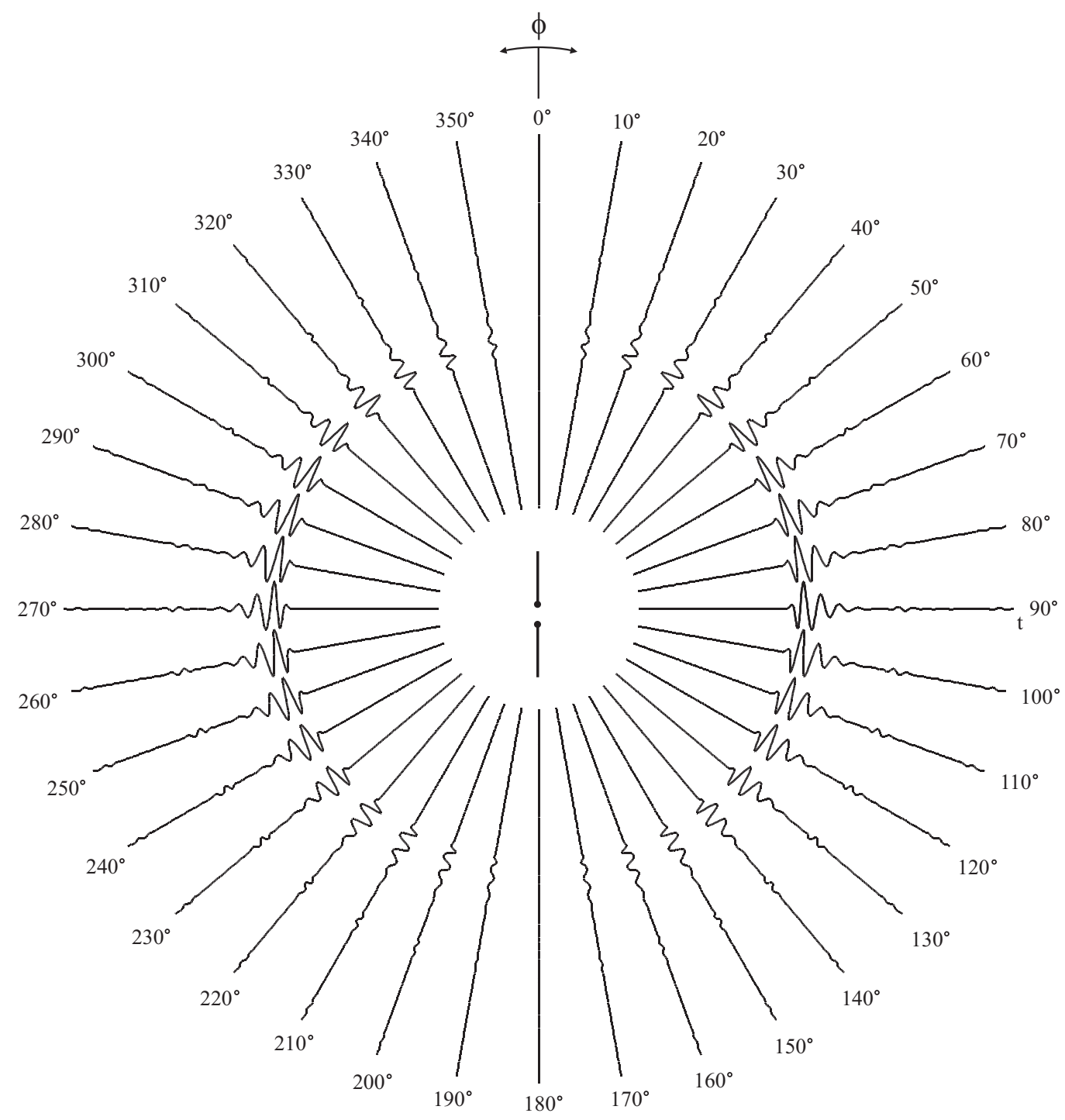

Figure 102: Received time-domain voltage by RX dipole in Fig. 101, as defined in Equation 43, with an unbalanced TX dipole excited with a $1 \mathrm{~V}$-peak differentiated Gaussian pulse with spectral peak at $3 \mathrm{GHz}$. The TX dipole was modeled with a feedline length of $11 \lambda / 4$ and was rotated in increments of $5^{\circ}$. 


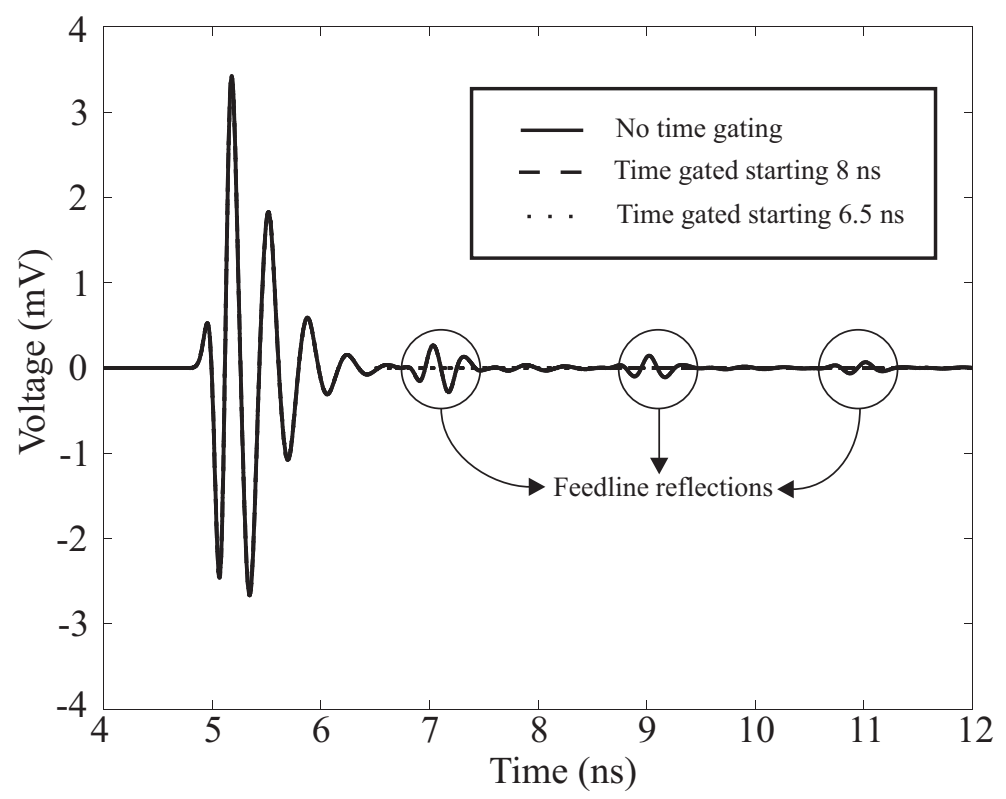

Figure 103: Time-domain voltage plots for unbalanced $5 \mathrm{~cm}$ dipole generated via NEC model in Fig. 101 at $\phi=90^{\circ}$. 

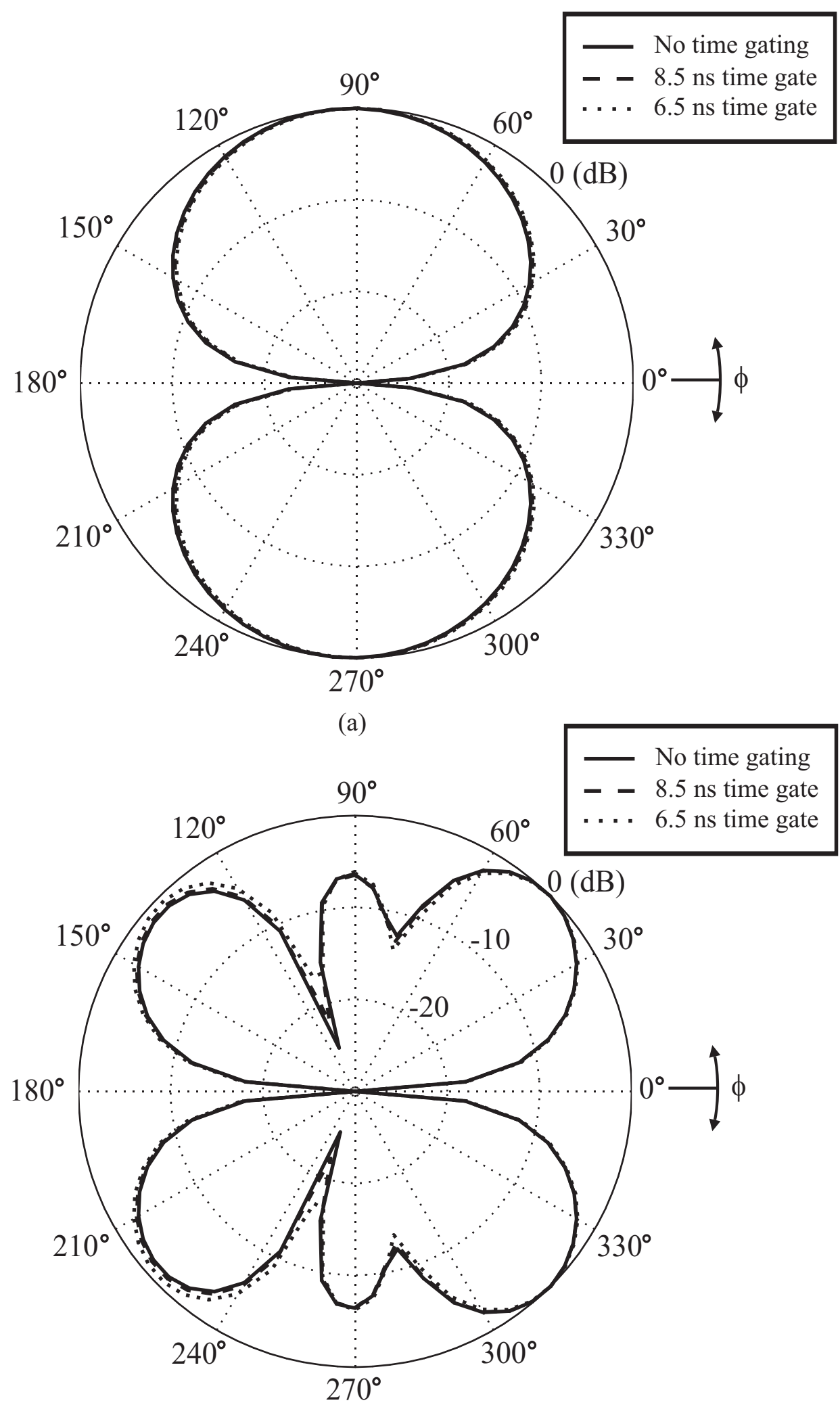

(b)

Figure 104: Normalized amplitude patterns $\left(\left|\mathrm{E}_{\phi}\right|\right)$ along azimuth plane generated from NEC model in Fig. 101 at (a) $\lambda / 2$ and (b) $3 \lambda / 2$. 


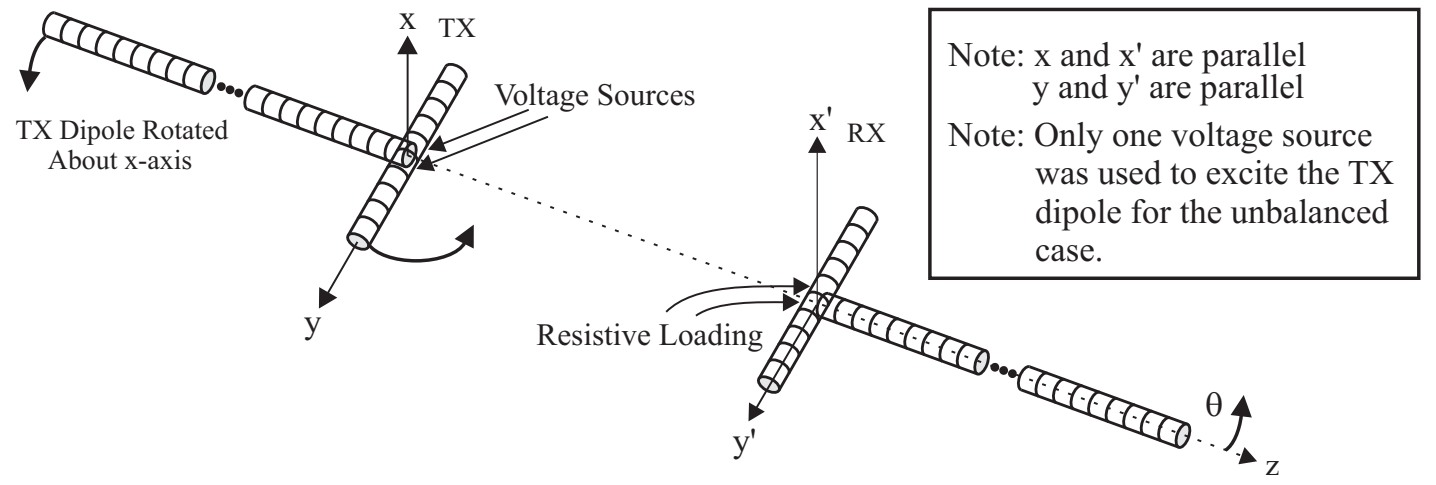

Figure 105: NEC model used to study the effect of time gating secondary reflections caused by unbalanced dipole feed on the frequency domain amplitude pattern along the elevation plane. 


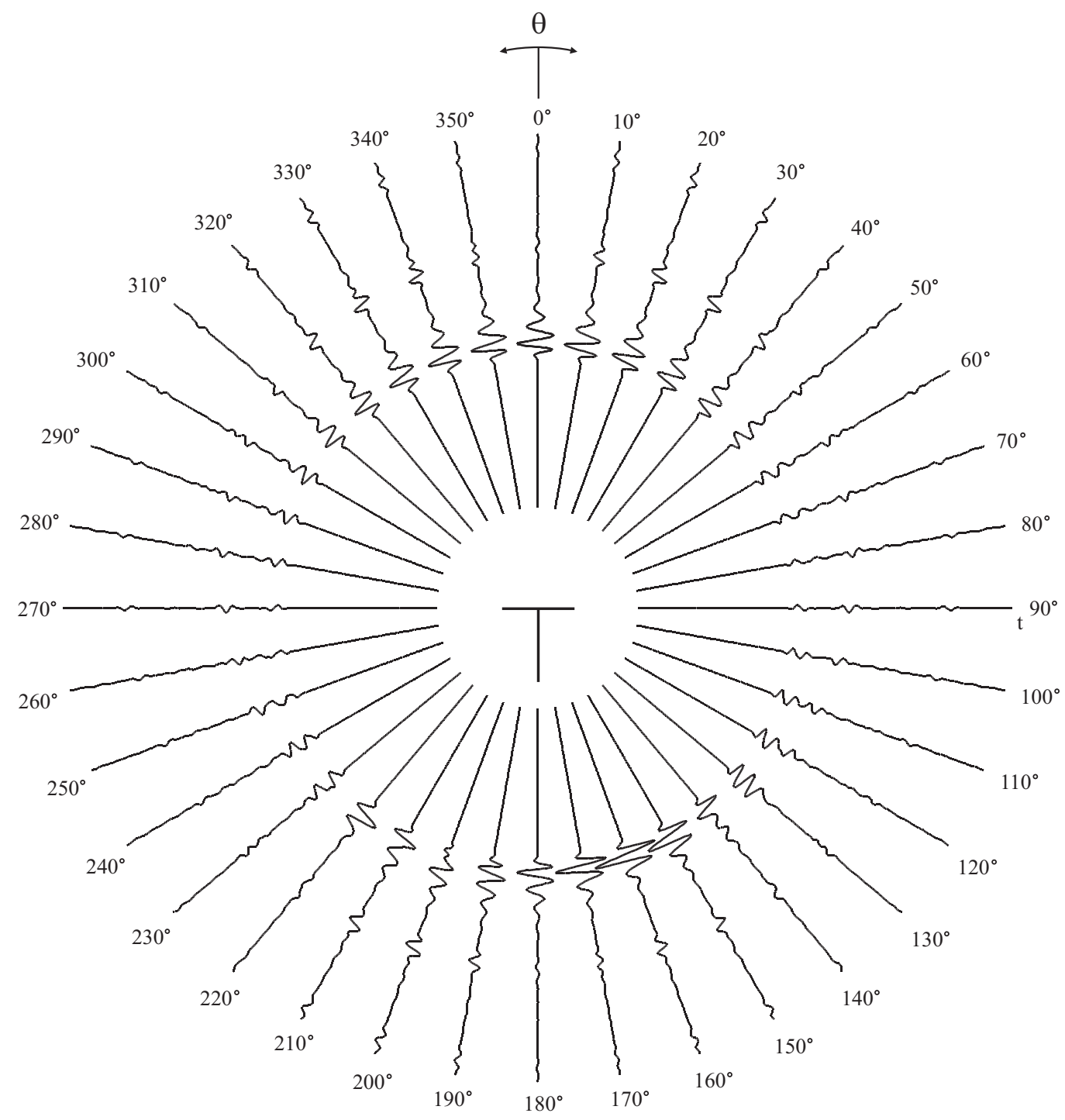

Figure 106: Received time-domain voltage by RX dipole in Fig. 105, as defined in Equation 43, with an unbalanced TX dipole excited with a $1 \mathrm{~V}$-peak differentiated Gaussian pulse with spectral peak at $3 \mathrm{GHz}$. The TX dipole was modeled with a feedline length of $11 \lambda / 4$ and was rotated in increments of $5^{\circ}$. 


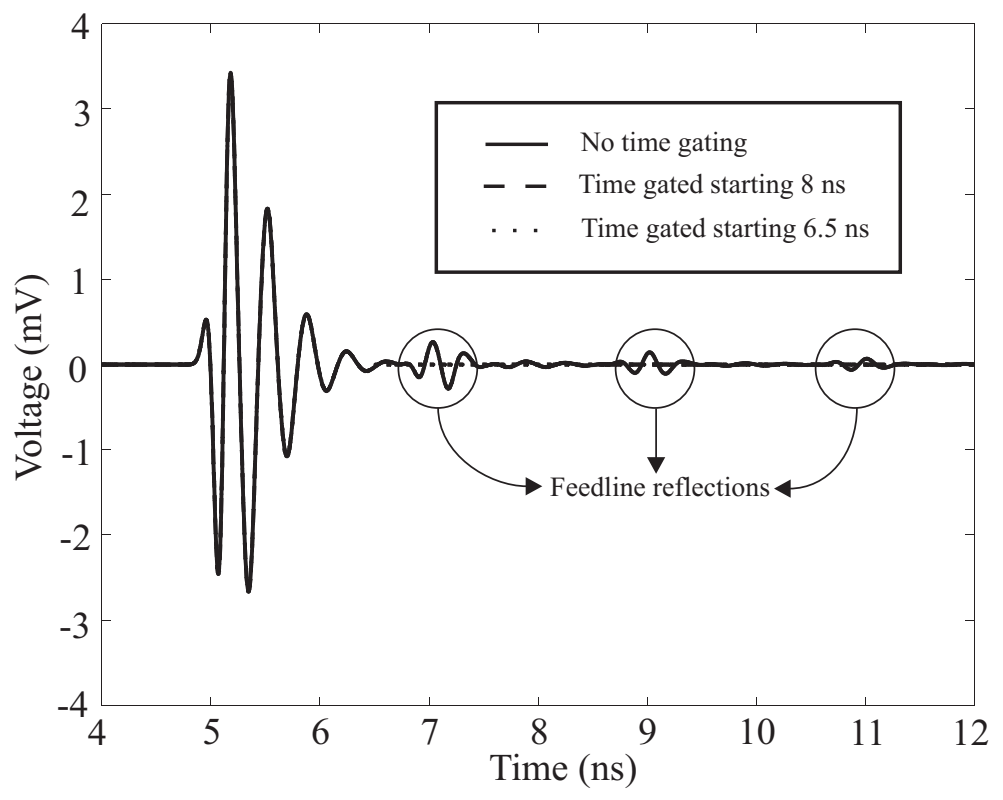

Figure 107: Time-domain voltage plots for unbalanced $5 \mathrm{~cm}$ dipole generated via NEC model in Fig. 105 at $\theta=0^{\circ}$.

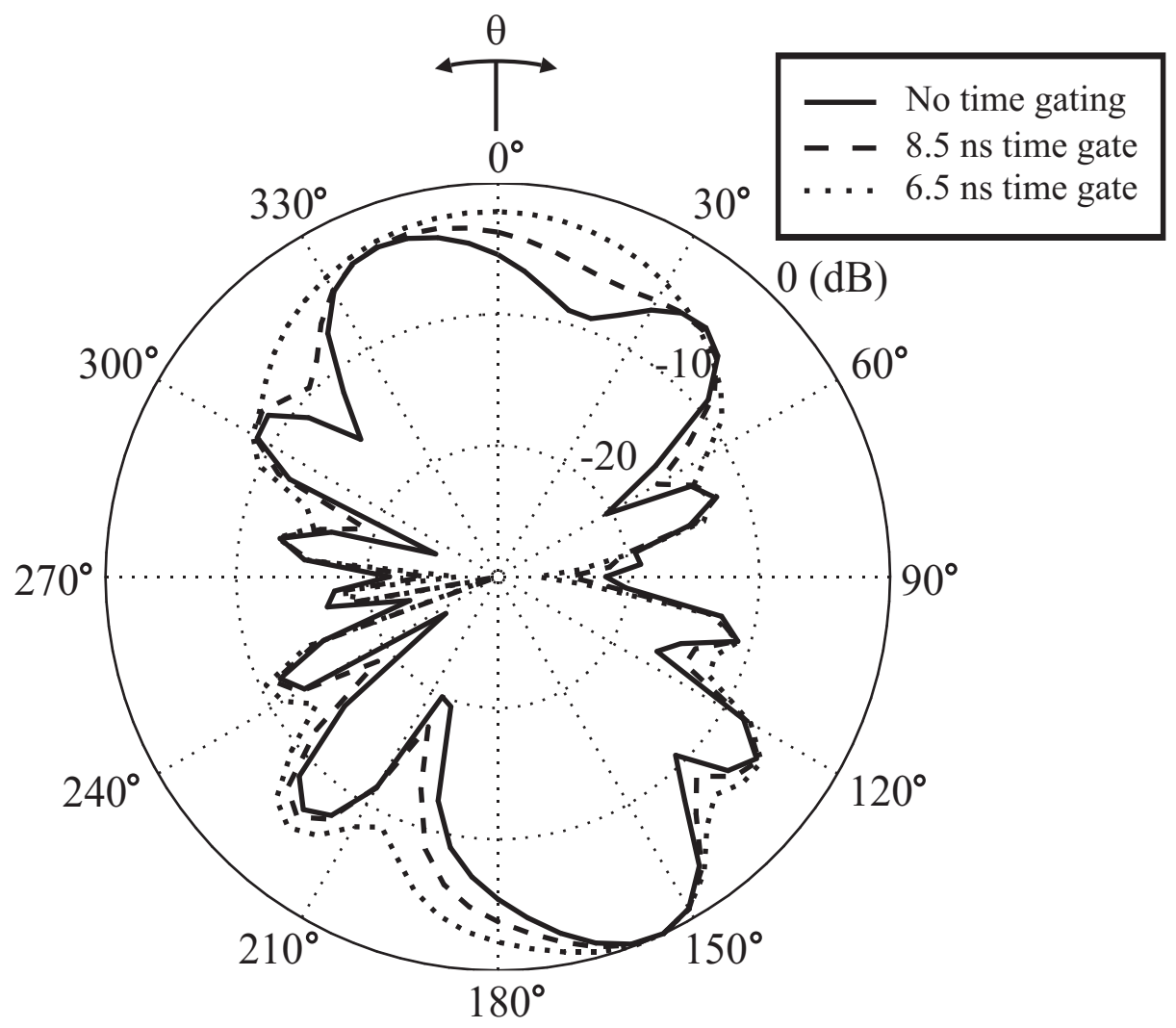

Figure 108: Normalized amplitude patterns $\left(\left|\mathrm{E}_{\theta}\right|\right)$ along elevation plane generated from NEC model in Fig. 105 at $\lambda / 2$. 


\subsubsection{Measuring Cross-Polarized Component}

As explained in Chapter 1, an unbalanced dipole feed induces a common-mode current along the feedline structure. This common-mode current causes the feedline to radiate. Radiation from the vertical feedline is vertically polarized, in contrast to the horizontally polarized field radiated by the arms of the dipole. Hence, if a receiving dipole has its arms oriented horizontally, the desired horizontal field component from the arms of the transmitting dipole is referred to as the co-polarized component, and the undesired vertical field component from the feedline is denoted as the cross-polarized component. A perfectly balanced dipole induces no current along the feedline, therefore, the cross-polarized field component is zero; the cross-polarized component of the radiated field of an unbalanced dipole is nonzero. While the presence of a cross-polarized component of the radiated field indicates an unbalanced feed, this section investigates whether the performance of a balun can be evaluated by measuring the cross-polarized component of the radiated field of a dipole fed with and without the balun.

To calculate the radiation from the feedline (cross-polarized component) of an unbalanced dipole fed with the double-y balun, the NEC model in Fig. 101 was modified as illustrated in Fig. 109, where the arms of the RX probe dipole were oriented vertically, parallel to the TX dipole's feedline. The dipoles were placed 1.5 meters apart, and the length of the TX dipole was chosen to be $11 \lambda / 4$. The TX dipole was rotated $360^{\circ}$ in increments of $5^{\circ}$, and the time-domain transfer function was obtained at each angle for the two dipoles.

The received time-domain voltage by RX dipole in Fig. 109, as defined in Equation 43, with a balanced TX dipole excited with a $1 \mathrm{~V}$-peak differentiated Gaussian pulse with spectral peak at $3 \mathrm{GHz}$, is illustrated in Fig. 110. The TX dipole was modeled with a feedline length of $11 \lambda / 4$ and was rotated in increments of $5^{\circ}$. Since the arms of the dipole are balanced, no current is induced along the feedline. Therefore, only 
the arms of the balanced dipole radiate. This radiation, however, is horizontally polarized, and is not received by the RX dipole oriented vertically in Fig. 109.

The received time-domain voltage by RX dipole in Fig. 109, as defined in Equation 43, with an unbalanced TX dipole excited with a 1 V-peak differentiated Gaussian pulse with spectral peak at $3 \mathrm{GHz}$, is illustrated in Fig. 111. From the time-domain pattern, it is seen that the unbalanced dipole radiates in all directions. Furthermore, this radiation is uniform in all directions; no nulls are present along a particular angle. In a practical measurement setup, a dipole is typically fed with a coaxial line. When the feed is unbalanced, the common-mode current induced along the coaxial line causes the feedline to radiate. The time-domain pattern in Fig. 111 was obtained via the NEC model in Fig. 109, with $\mathrm{L}_{f}=11 \lambda / 4$. However, in practice, the coaxial feedline may be much longer than $11 \lambda / 4$, as explained in Section 3.3. The effect of varying the feedline length on the cross-polarized field component of an unbalanced dipole is studied next.

The NEC model in Fig. 101 was modified by extending the feedline length, $\mathrm{L}_{f}$, from $11 \lambda / 4$ to $21 \lambda / 4$ and placing a Wu-King taper at the end of the feedline. This was done to approximate an infinite feedline. The Wu-King profile was that obtained in Section 3.3. The resulting time-domain pattern is illustrated in Fig. 112. Again, for the unbalanced TX dipole in Fig. 109, the cross-polarized component of the radiated field is present at all angles. However, the patterns in Fig. 112 clearly differ from those in Fig. 111. An expanded view comparing both time-domain patterns at $0^{\circ}$ is illustrated in Fig. 113. It is seen that the received pulses at 6, 8, 10, and 12 nanoseconds are not present with the unbalanced dipole with a long feedline. These pulses, spaced two nanoseconds apart, result from travelling down the feedline and radiating at the end of the feedline. Hence, these pulses do not appear for the unbalanced dipole with an infinitely long feedline, modeled using the Wu-King profile. However, it is interesting to note that a radiated pulse from the feedline appears near 
$7 \mathrm{~ns}$ with the $\mathrm{Wu}-\mathrm{King}$ loading. This pulse results from radiation along the $\mathrm{Wu}-\mathrm{King}$ taper. The current along the taper experiences a deceleration, therefore resulting in radiation that is spread in time, as seen in Fig. 113. It is also interesting to note that the pre-pulse is identical in both cases, with a finite length feedline and with an infinite length feedline. Ideally, the magnitude of this pre-pulse could be used to determine the effectiveness of a balun (the better the balance, the smaller the magnitude of the pre-pulse). However, the model in Fig. 101 is a simple model, and does not model the double-y balun or the sleeve balun physically. Therefore, the pre-pulse waveform will differ for a dipole fed with either a double-y balun or a sleeve balun.

Figure 114 illustrates the normalized amplitude patterns for both cases at $\lambda / 2$. It is clearly seen that the cross-polarized field component is dependent on the length of the feedline. That is, while the presence of a cross-polarized component of the radiated field from a dipole indicates an unbalanced feed, measuring this field component does not provide insight into the degree of balancing achieved by a balun. This is because the strength of this field component is sensitive to the feedline length. Results obtained in this chapter are used to construct a new antenna pattern measurement system in subsequent chapters. The performance of the double-y balun is investigated via antenna patterns conducted using the new pattern measurement system. 


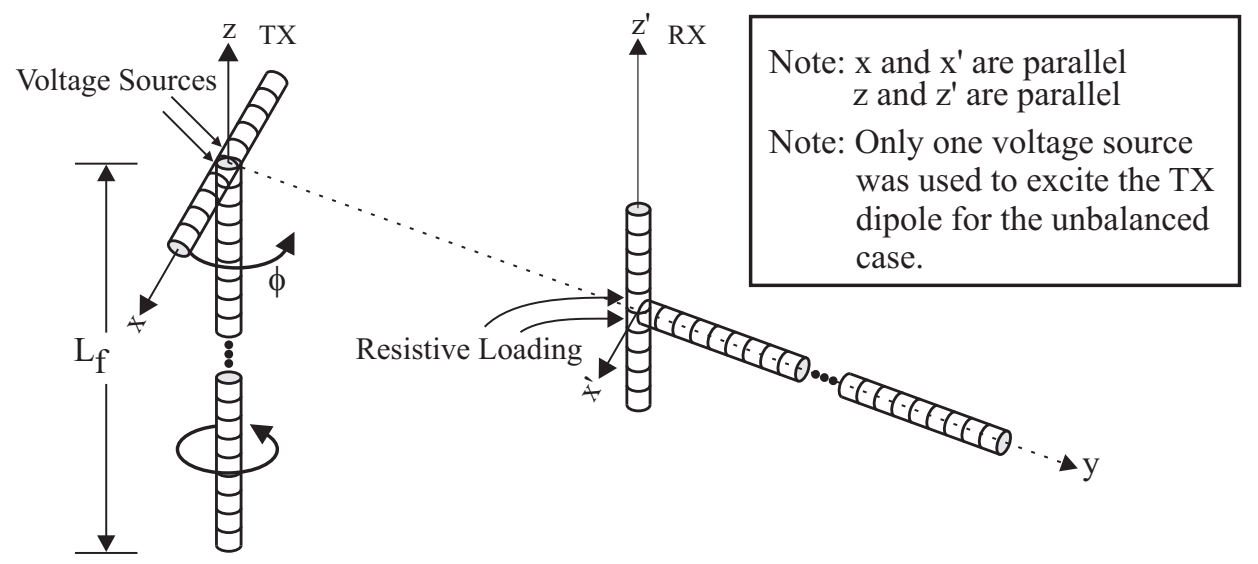

Figure 109: NEC model used to study the cross-polarized component of the radiated field from an unbalanced dipole. 


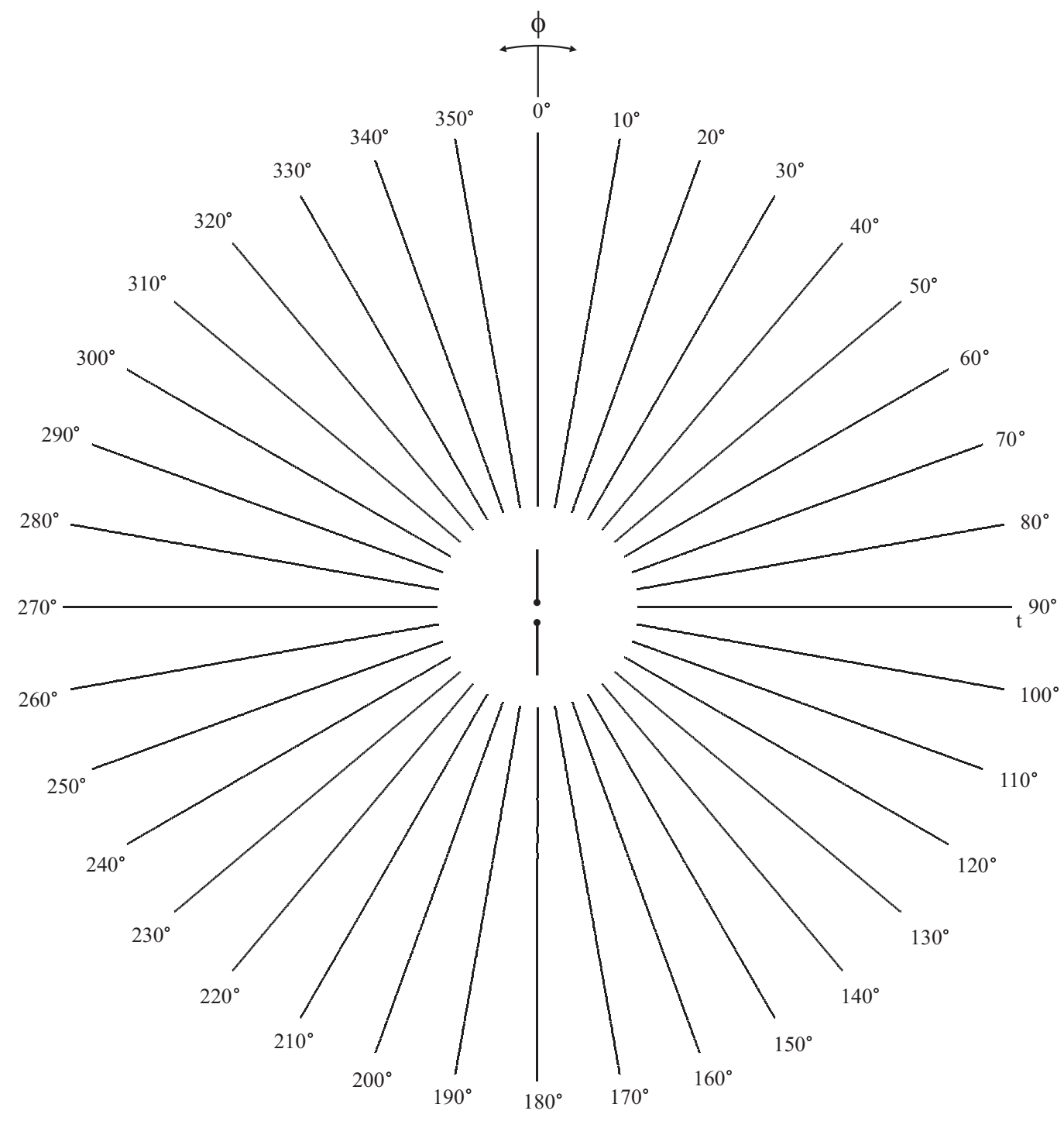

Figure 110: Received time-domain voltage by RX dipole in Fig. 109, as defined in Equation 43, with a balanced TX dipole excited with a $1 \mathrm{~V}$-peak differentiated Gaussian pulse with spectral peak at $3 \mathrm{GHz}$. The TX dipole was modeled with a feedline length of $11 \lambda / 4$ and was rotated in increments of $5^{\circ}$. 


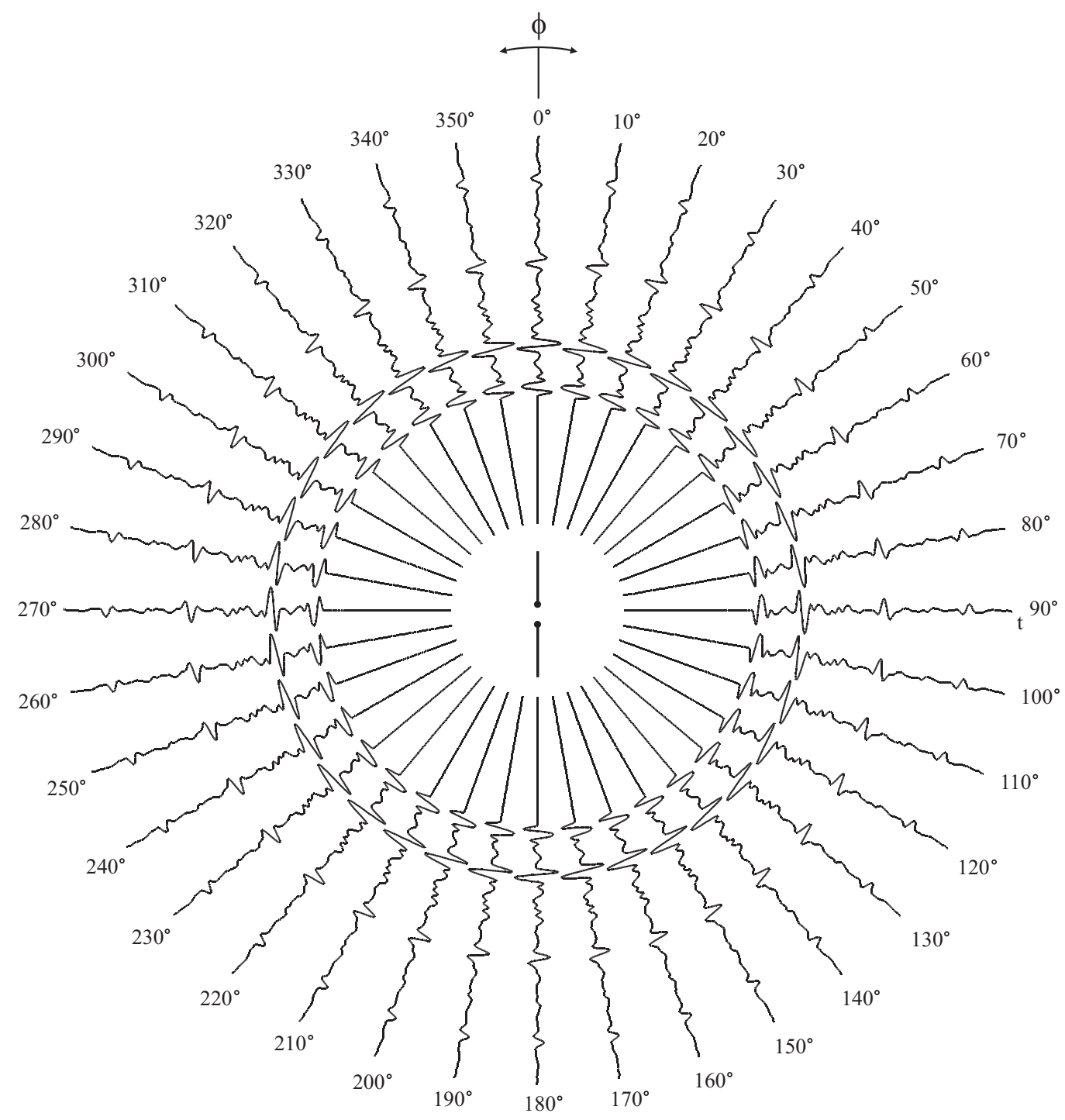

Figure 111: Received time-domain voltage by RX dipole in Fig. 109, as defined in Equation 43, with an unbalanced TX dipole excited with a $1 \mathrm{~V}$-peak differentiated Gaussian pulse with spectral peak at $3 \mathrm{GHz}$. The TX dipole was modeled with a feedline length of $11 \lambda / 4$ and was rotated in increments of $5^{\circ}$. 


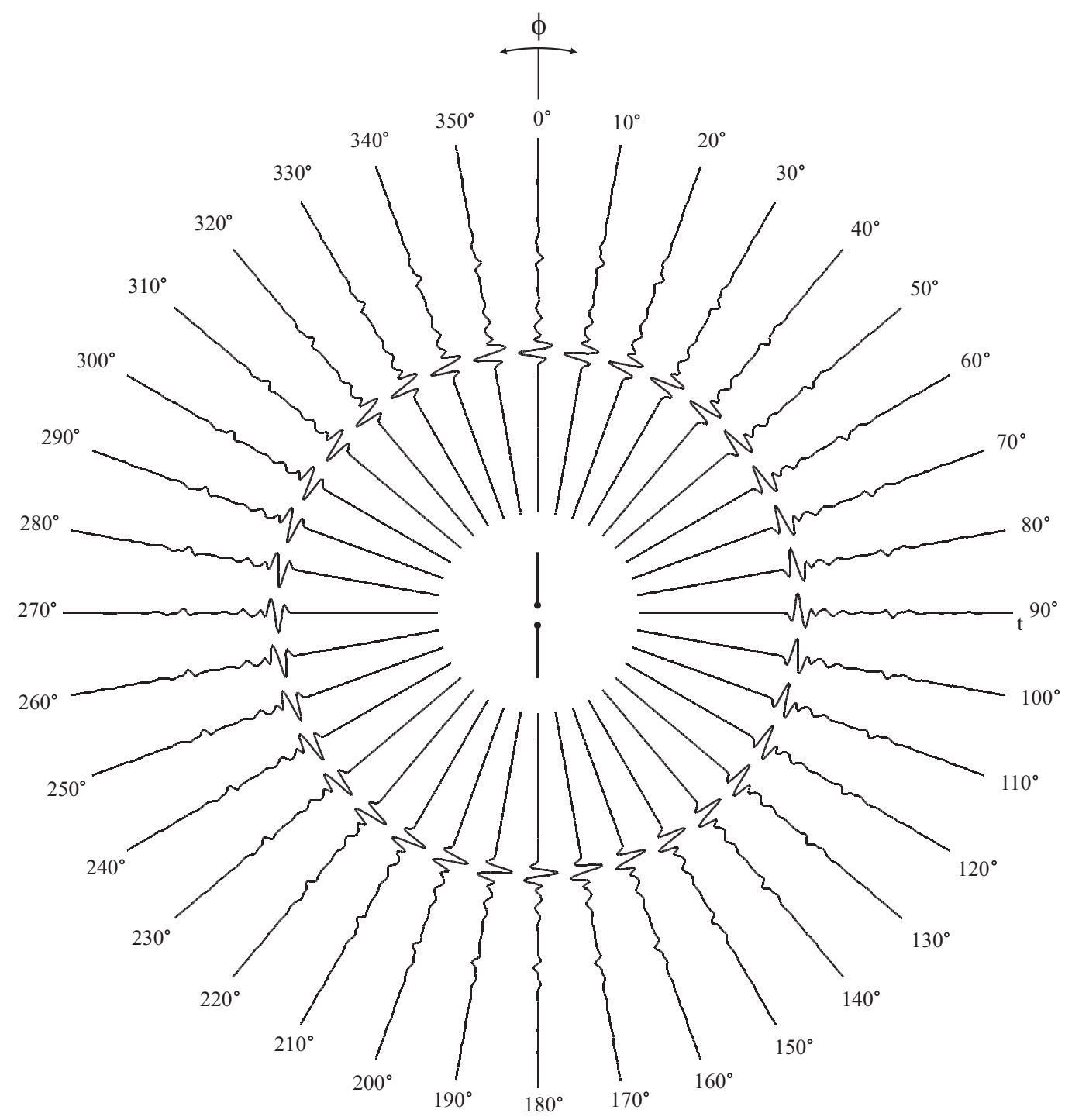

Figure 112: Received time-domain voltage by RX dipole in Fig. 109, as defined in Equation 43, with an unbalanced TX dipole excited with a $1 \mathrm{~V}$-peak differentiated Gaussian pulse with spectral peak at $3 \mathrm{GHz}$. The TX was rotated in increments of $5^{\circ}$ with $\mathrm{Wu}$-King loading along the feedline. 


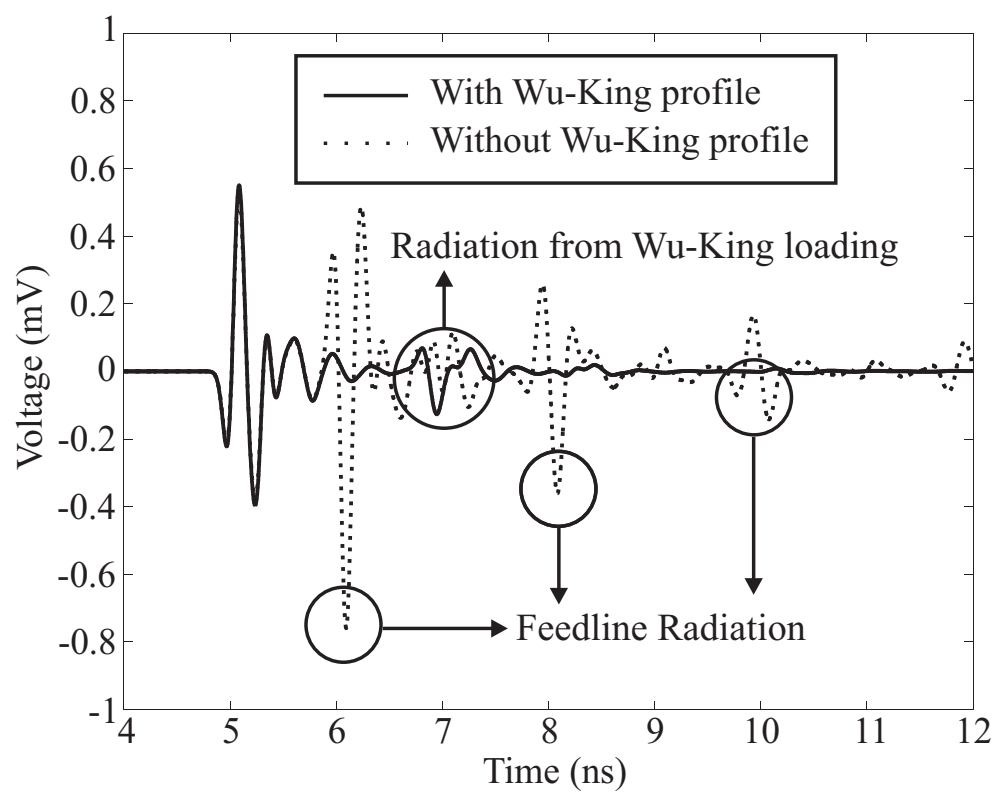

Figure 113: Expanded view of time-domain patterns from Figs. 111 and 112, at $0^{\circ}$.

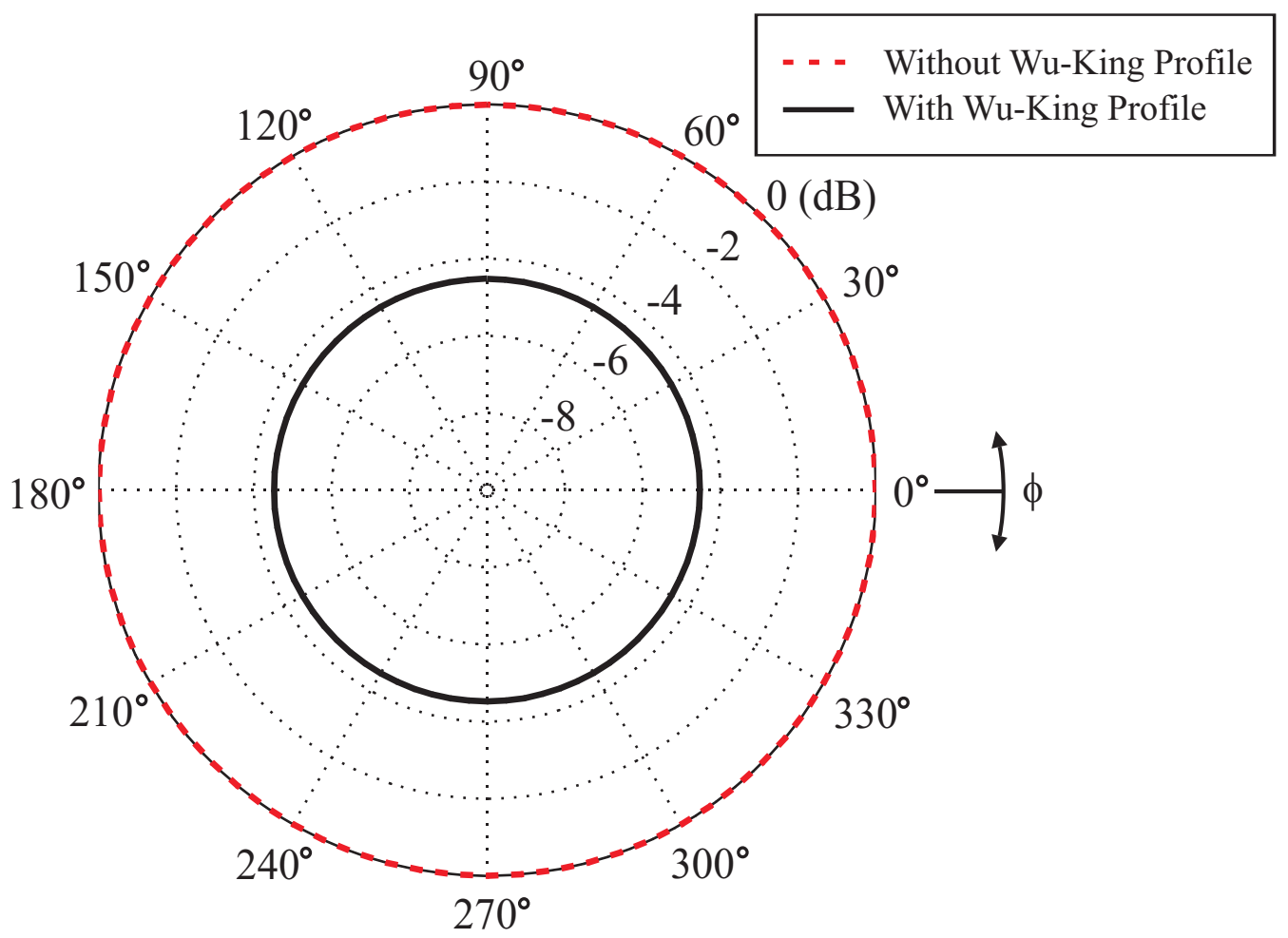

Figure 114: Normalized amplitude pattern computed via NEC model in Fig. 109 for two feedline lengths. 


\section{CHAPTER IV}

\section{OPTICAL LINK}

\subsection{Motivation}

Figure 115 illustrates a commonly used experimental setup for conducting antenna pattern measurements. If symmetric antennas requiring a balanced feed are used in the experimental setup, and the arms of the antenna are not perfectly balanced, a common-mode current is induced along the coaxial feedline. Common-mode current along the feedline (e.g. coaxial line) causes the feed structure to radiate in addition to the antenna. The strength of this common-mode current varies with feedline length, as discussed in the previous chapter.

In Section 3.2, it was shown via NEC models that the amplitude pattern of a dipole along the elevation plane (elevation plane is defined in Fig. 84 in Section 3.2) is significantly affected when the feed is unbalanced. In a practical measurement setup such as the one illustrated in Fig. 115, it is difficult to determine how much of the coaxial line radiates due to an unbalanced feed. If the coaxial cable is radiating, a small movement or shift in the cables could alter the measured antenna patterns significantly. We see that in order to measure the effectiveness of a balun via antenna pattern measurements, a sound measurement system is required, one that allows reproducible results to be obtained. It might prove difficult to obtain consistent pattern measurements with the setup in Fig. 115 if the feed is not perfectly balanced. Radiation from the coaxial feedline needs to be contained somehow.

To measure the radiation pattern along the elevation plane of an antenna under test (AUT) more accurately, a new experimental setup was constructed, as illustrated in Fig. 116, with an optical link replacing the long coaxial feedline connecting the 


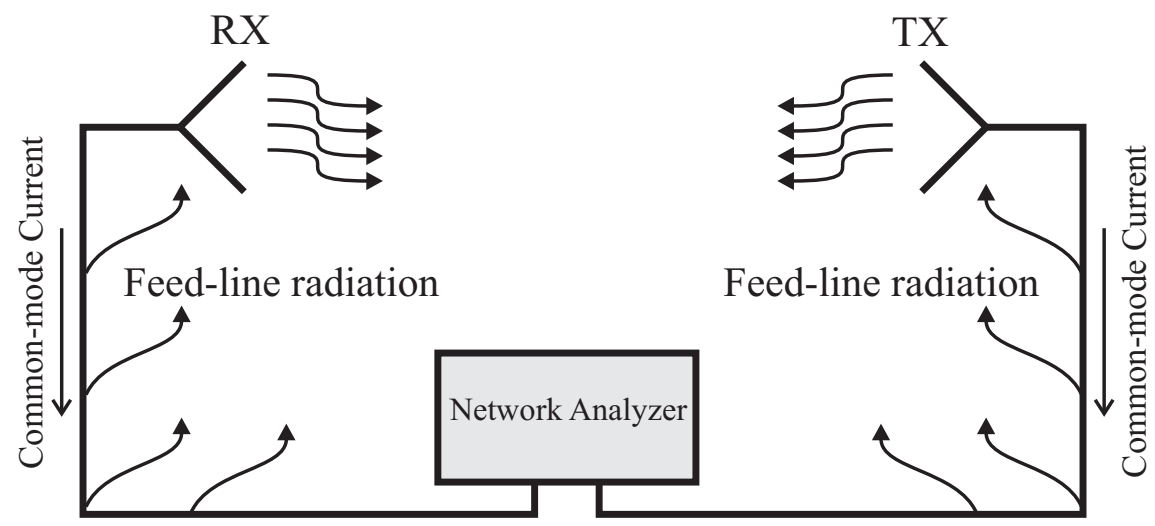

Figure 115: Effect of common-mode currents in an antenna measurement setup.

AUT. The optical link consists of a laser modulator (LM), a laser receiver (LR), and fiber optic cable linking both units. The use of a fiber link confines radiation from common-mode currents to the reduced feedline and the LR unit. Therefore, if the balun is not properly balancing the antenna feed, radiation from the reduced feedline can be accurately measured with the new experimental setup via antenna pattern measurements. In the time domain, the finite length feedline, consisting of the truncated coaxial feedline and the LR unit, enables feedline reflections to exist when the antenna feed is unbalanced, thus allowing the patterns of a balanced and unbalanced feed to be discerned with improved accuracy. Surrounding the RX antenna (stationary probe) with microwave absorbers reduces feedline radiation from the RX probe antenna from coupling into measurement results.

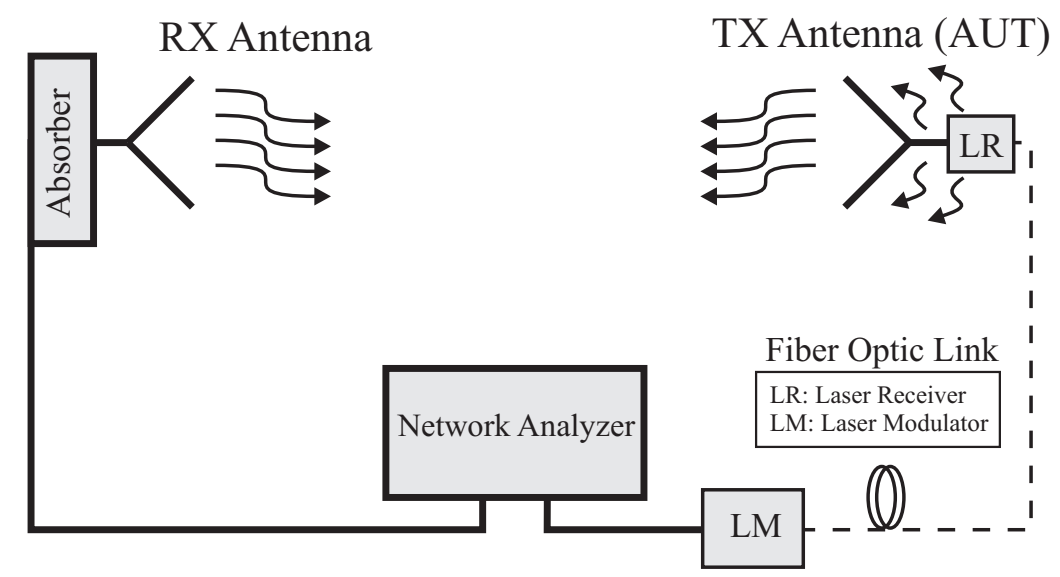

Figure 116: New experimental setup with optical link replacing long coaxial feedline. 


\subsection{Laser Modulator Unit (LM)}

The laser modulator unit, illustrated in Fig. 117, consists of an Alcatel 1916LMM laser module with integrated electro-absorption modulator and the associated components necessary to bias and monitor this module. The 1916LMM module is illustrated in Fig. 118. The RF board housing the laser module and laser driver chip was fabricated on Taconic's CER-10 substrate $\left(\varepsilon_{r}=10.0+/-0.5\right.$ and thickness $=45$ mils $)$. The choice of substrate was dictated by receiver characteristics and is discussed in the next section. The widths of the RF traces for the modulator (pins 11, 12, and 13 of laser module) were synthesized (synthesis was carried out using HP ADS) to be 60 mils (upper design frequency was chosen to be $20 \mathrm{GHz}$ ). The widths of the traces carrying the thermoelectric cooler (TEC) control signals were chosen to be 70 mils in order to handle the higher current amperage from the TEC unit. The module was powered using the external T450-P5P $5 \mathrm{~V}$ transformer from Digi-Key.

The laser was biased at $~ 80 \mathrm{~mA}$ using the HY6330 constant current laser diode driver from Hytek Microsystems. An external protection circuit was added, as illustrated in Fig. 119 to limit the photodiode voltage to $1.8 \mathrm{~V}$ as well as to prevent transient signals from reaching the photodiode. Two 1N4148 diodes were connected in series to limit the voltage (two sets of diodes limit positive and negative voltages) and a $0.01 \mu \mathrm{F}$ capacitor was used to filter transient signals.

The laser module's temperature was controlled by using the HY5650 TEC controller chip. The chip was mounted on an HY5650 evaluation board. A heat sink was mounted on the TEC controller to properly dissipate heat from the chip. The TEC current was limited to $1 \mathrm{~A}$ and the laser module's temperature was set to be 25 ${ }^{\circ} \mathrm{C}$ by using a $10 \mathrm{k} \Omega$ temperature set resistor. The laser module's internal thermistor was used to provide the reference temperature. Table 3 summarizes the optical characteristics of the laser module. 


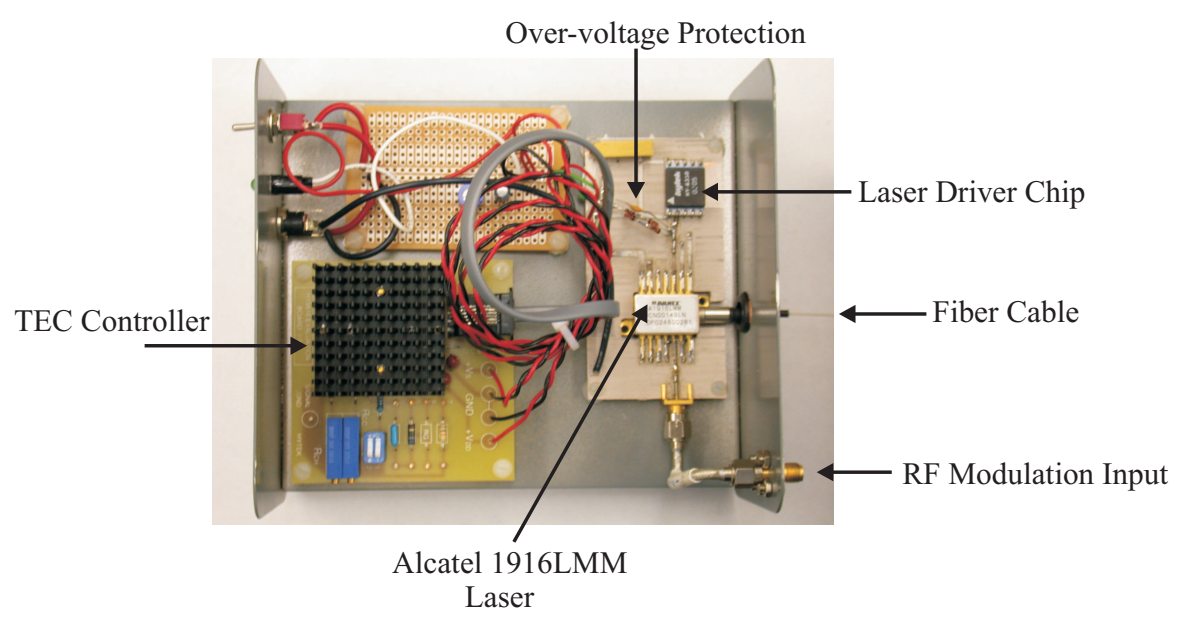

Figure 117: Laser modulator unit.

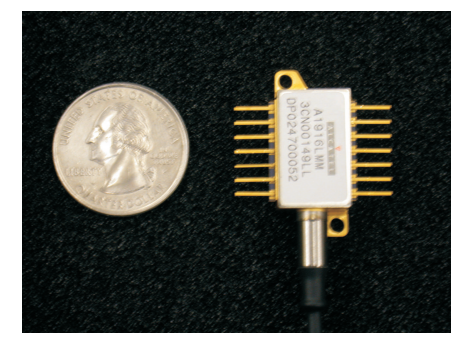

Figure 118: Alcatel 1916LMM laser module with integrated electro-absorption modulator.

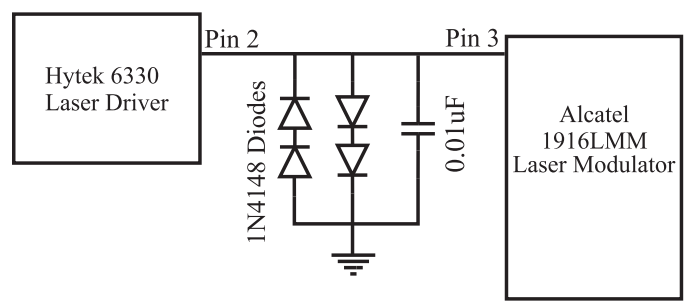

Figure 119: Bias protection circuit for laser diode.

\subsection{Laser Receiver Unit (LR)}

The laser receiver unit is illustrated in Fig. 120. The receiver unit consists of the Alcatel $191510 \mathrm{~Gb} / \mathrm{s}$ receiver module, illustrated in Fig. 121, associated biasing circuitry, and RF amplifiers. Figure 122 illustrates a system level diagram of the receiver circuitry. The receiver unit is powered by three Kokam lithium polymer (Li Poly) cells connected in series. Each Li Poly cell cell has a $3.7 \mathrm{~V}$ nominal voltage and 4.2 $\mathrm{V}$ maximum voltage. Each cell has an absolute minimum voltage of $2.5 \mathrm{~V}$ and 
Table 3: Optical characteristics of the Alcatel 1916LMM laser module [1].

\begin{tabular}{|c||c|c|}
\hline \multicolumn{1}{|c||}{ Parameter } & Value & Units \\
\hline Operating Current & 80 & $\mathrm{~mA}$ \\
\hline Optical Output Power & -1.47 & $\mathrm{dBm}$ \\
\hline Peak Emission Wavelength & 1558.17 & $\mathrm{~nm}$ \\
\hline Modulation Bias Voltage & 0 & $\mathrm{~V}$ \\
\hline Modulation Voltage & 1.0 & $\mathrm{~V}_{p-p}$ \\
\hline TEC Current & 1 & $\mathrm{~A}$ \\
\hline+ Operating Current & 80 & $\mathrm{~mA}$ \\
\hline Thermistor Resistance at $\mathrm{T}=25^{\circ} \mathrm{C}$ & 10 & $\mathrm{k} \Omega$ \\
\hline
\end{tabular}

has a 1020 mAH capacity. Terminal connectors were mounted on the receiver board to connect to the battery.

The battery protection circuit consists of a Max1665V and IRF9956 dual n-channel MOSFET IC, as illustrated in Fig. 123. The Max1665V provides protection against undervoltage for 3-cell lithium-ion and Li Poly battery packs. If the voltage across any cell falls below $+2.5 \mathrm{~V}$, the Max1665V turns off the discharge MOSFET (external IRF9956 IC) connected to pin 2, thereby preventing further discharge of the battery pack.

The battery monitoring circuit is illustrated in Fig. 124. The circuit powers the green LED when the receiver is turned on. Should the battery voltage fall below the minimum allowable cell voltage, the red LED turns on, indicating a low battery status. The green LED has a typical forward voltage of $2.2 \mathrm{~V}$ and the red LED has a typical forward voltage of $2.0 \mathrm{~V}$; both LEDs had a rated current of $20 \mathrm{~mA}$.

A D1 International 3A14 DC-DC converter was used to step down the input voltage from the LiPoly battery pack and provide +/- 5 VDC to the Alcatel 1915 module and RF amplifiers. The 3A14 accepts input voltages in the range of 9-18 VDC and provides an output voltage of +/- 5 VDC with an output current of +/$300 \mathrm{~mA}$. The DC-DC converter operates with an efficiency of $73 \%$. 


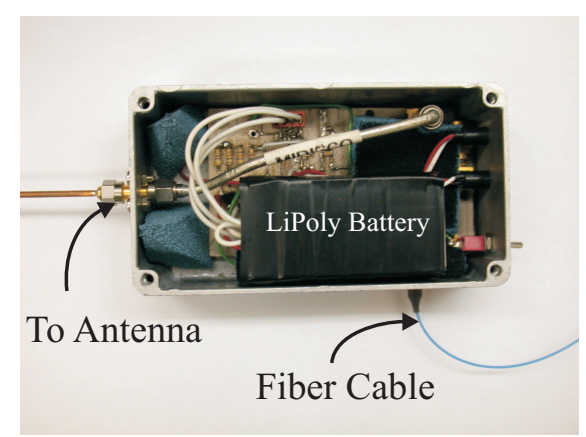

(a)

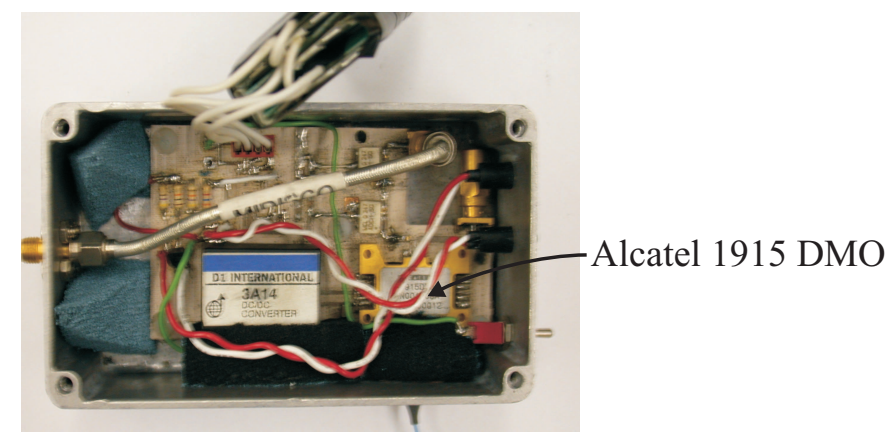

(b)

Figure 120: Illustration of laser receiver (LR) unit (a) with enclosed LiPoly battery and (b) with battery back removed to view internal circuitry.

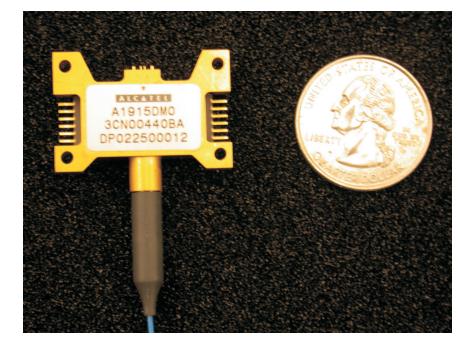

Figure 121: Alcatel 1915DMO receiver module.

The Alcatel 1915 module contains a InGaAs PIN photodiode and a SiGe transimpedance amplifier. Table 4 provides optical and electrical characteristics of the 1915 module. Figure 125 illustrates an expanded view of the differential output traces from the Alcatel 1915 module. Each of the two output pins from the module are designed to drive individual $50 \Omega$ loads or a $100 \Omega$ differential output. Since a single-ended output is required from the receiver unit, one of the differential terminals was terminated with a broadband $50 \Omega$ matched load. To achieve practical $50 \Omega$ 


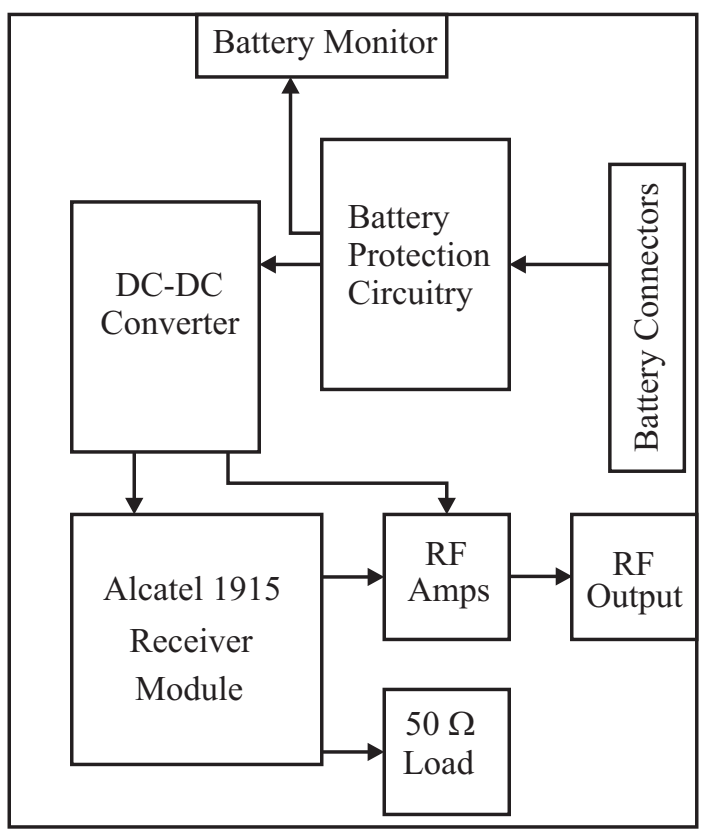

Figure 122: System level diagram of receiver circuitry.

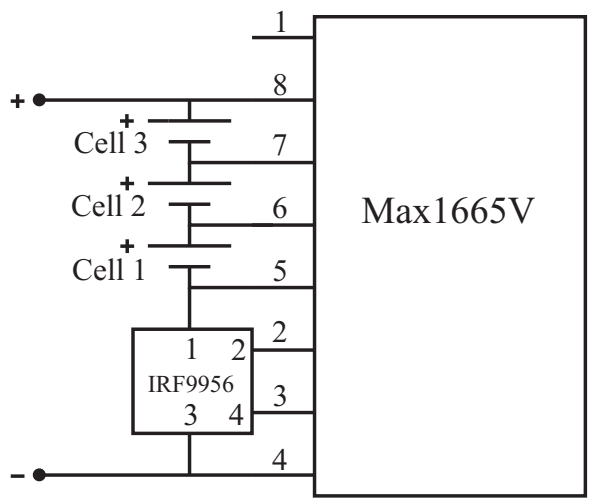

(a)

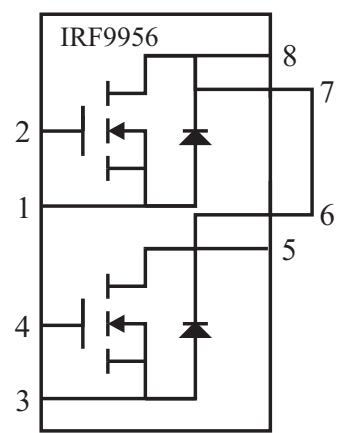

(b)

Figure 123: Operating circuit and pinout of (a) Max1665V and (b) IRF9556 ICs.

transmission lines for the given trace and gap widths, a high permittivity substrate was required. Hence, Taconic's CER-10 substrate $\left(\varepsilon_{r}=10.0+/-0.5\right.$ and thickness $=45$ mils) was chosen to be the substrate for the receiver board.

The RF traces for the differential output from the 1915DMO module were synthesized using ADS. The widths of the RF traces were synthesized to be 47 mils (upper frequency of $10 \mathrm{GHz}$ ) over 45 mil thick CER-10 substrate. However, the output pins from the 1915 module are spaced so close to each other, as illustrated in Fig. 125b, 


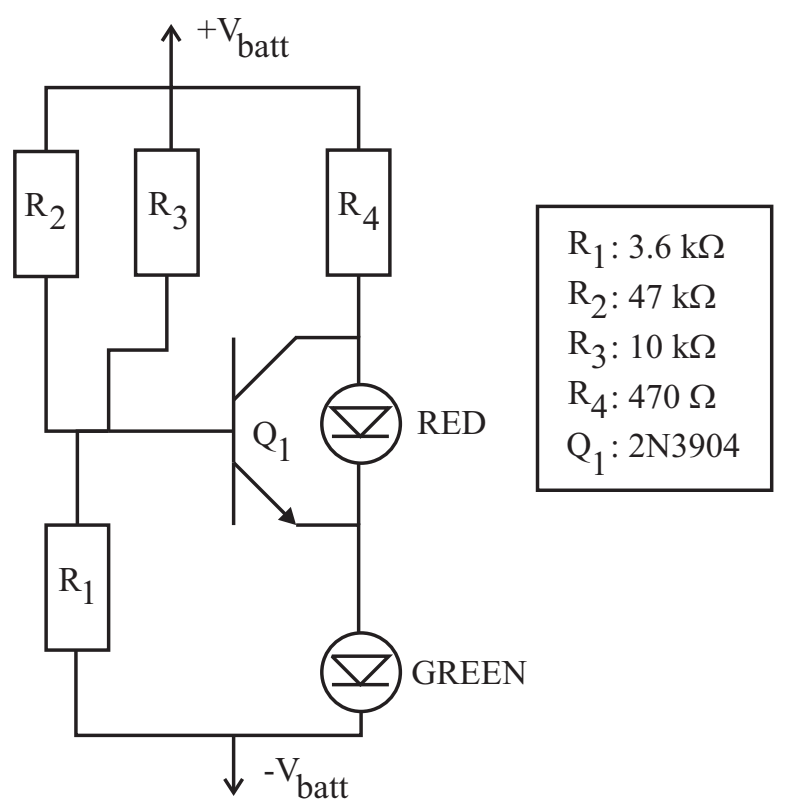

Figure 124: Battery monitoring circuitry.

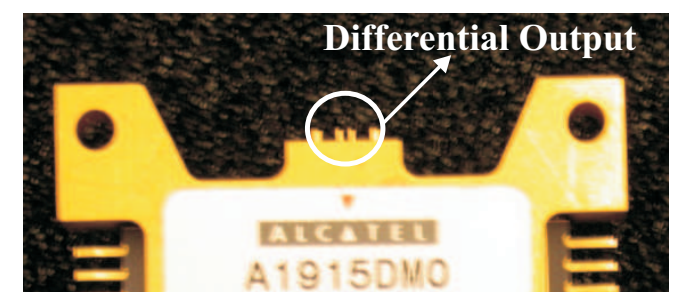

(a)

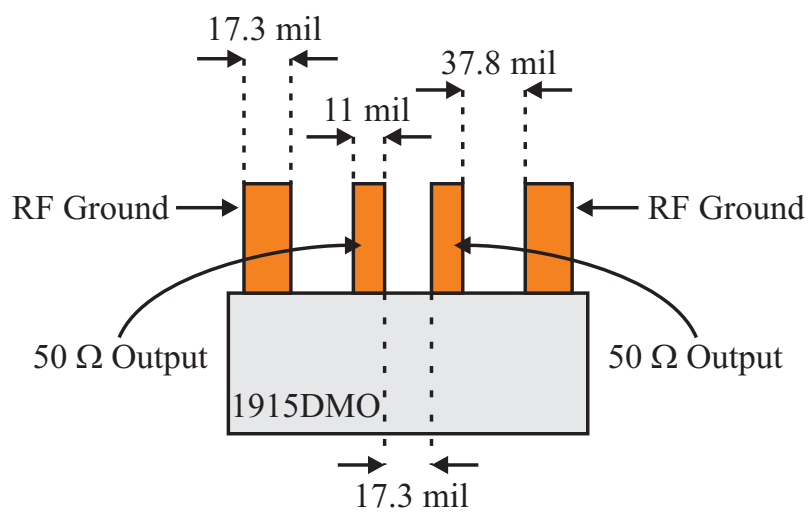

(b)

Figure 125: Illustration of (a) expanded view of differential output pins from 1915 module and (b) dimensions of output pins. 
Table 4: Optical and electrical characteristics of the Alcatel 1915 DMO receiver module [2].

\begin{tabular}{|c||c|c|c|c|}
\hline Parameter & Min & Typical & Max & Units \\
\hline PIN responsivity & 0.8 & 0.85 & - & $\mathrm{A} / \mathrm{W}$ \\
\hline Receiver Optical Sensitivity & - & - & -18 & $\mathrm{dBm}$ \\
\hline Receiver Optical Overload & 0 & 2 & - & $\mathrm{dBm}$ \\
\hline PIN Supply Voltage & 4.8 & 5.0 & 5.2 & $\mathrm{~V}$ \\
\hline TIA Power Supply Voltage & -5.4 & -5.2 & -5.0 & $\mathrm{~V}$ \\
\hline TIA Power Supply Current & - & 60 & 90 & $\mathrm{~mA}$ \\
\hline Transimpedance & 400 & 450 & - & $\Omega$ \\
\hline - 3dB Bandwidth (from $200 \mathrm{MHz}$ ) & 9 & 11 & - & $\mathrm{GHz}$ \\
\hline
\end{tabular}

that microstrip lines connected to these pins become coupled transmission lines. Because of the coupling between the lines, the section of transmission line designed to connect to these pins cannot be designed using equations for a microstrip. Therefore, transmission lines designed to connect to the output pins of the 1915 module, were synthesized by using a coupled microstrip model in ADS. The coupled section was then flanged out to decouple the lines into microstrip lines. The ADS model is illustrated in Fig. 126.

Figure 127 illustrates the insertion loss of the microstrip section designed using ADS and illustrated in Fig. 126. The model was simulated as a three port network; ideally both $\mathrm{s}_{21}$ and $\mathrm{s}_{31}$ should have an insertion loss of $3 \mathrm{~dB}$, since the input power is split equally between both ports. From the ADS simulation results, it is seen that the designed transmission line section, consisting of coupled microstrip lines and microstrip lines, exhibits an additional insertion loss of only $0.15 \mathrm{~dB}$ from $1 \mathrm{GHz}$ to $10 \mathrm{GHz}$.

Two ERA-1SM broadband monolithic amplifiers were used to boost the power of the RF signal from the 1915 module to above $0 \mathrm{dBm}$. Each amplifier stage was designed as illustrated in Fig. 128. An ADCH-80A super wide-band choke RF choke was used as the RF choke. The ERA amplifier was biased using a $35 \Omega$ chip resistor. 


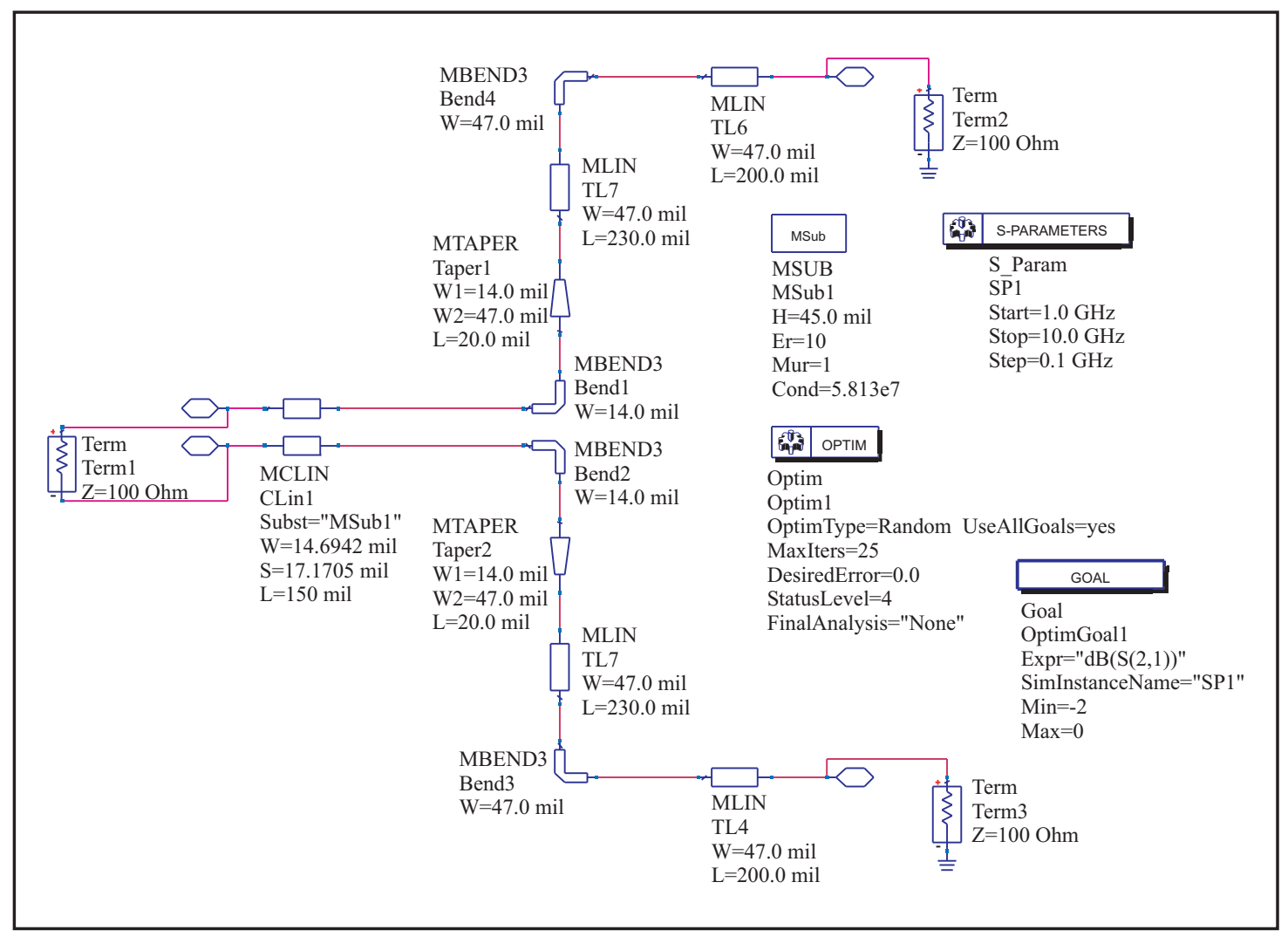

Figure 126: Design of RF traces for output from 1915DMO module using ADS.

DC blocking was achieved by using C08BLBB1X broadband DC block capacitors, manufactured by Dielectric Laboratories. A $1 \mathrm{nF}$ bypass capacitor was used to present a low impedance path to ground for any signal that gets past the RF choke. Power supply bypass capacitors with values of $1 \mathrm{uF}, 0.1 \mathrm{uF}$, and $1 \mathrm{nF}$ were used in parallel. The power supply consisted of 5 VDC provided from the DC/DC converter chip. Figure 129 illustrates the measured output power from the LR unit. The sharp resonances after $6 \mathrm{GHz}$ are likely caused by the LM unit, since the 1916 module's cutoff is $4 \mathrm{GHz}$. The low pass behavior of the power plot could result from poor RF grounding as well as the variable gain of the ERA amplifier versus frequency. From the measured power, it is seen that sufficient power is available up to $6 \mathrm{GHz}$ to conduct pattern measurements with the optical link. 


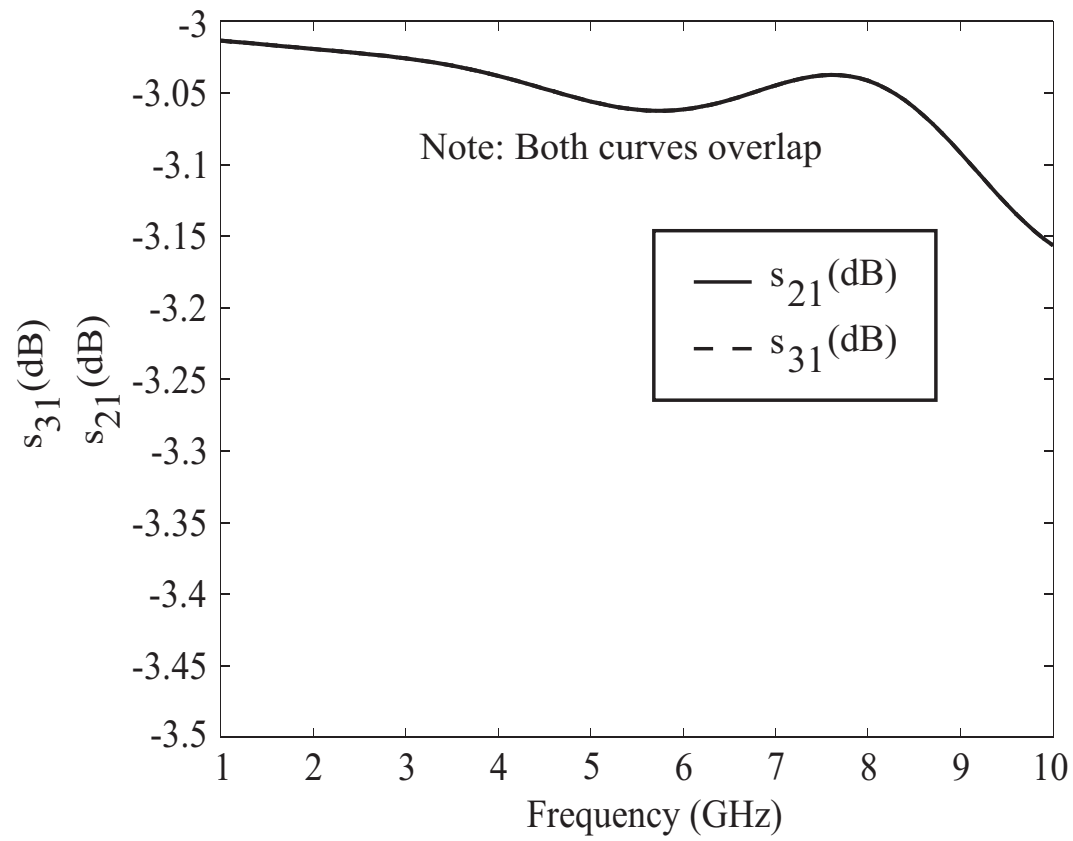

Figure 127: Insertion loss of microstrip section obtained via ADS model illustrated in Fig. 126.

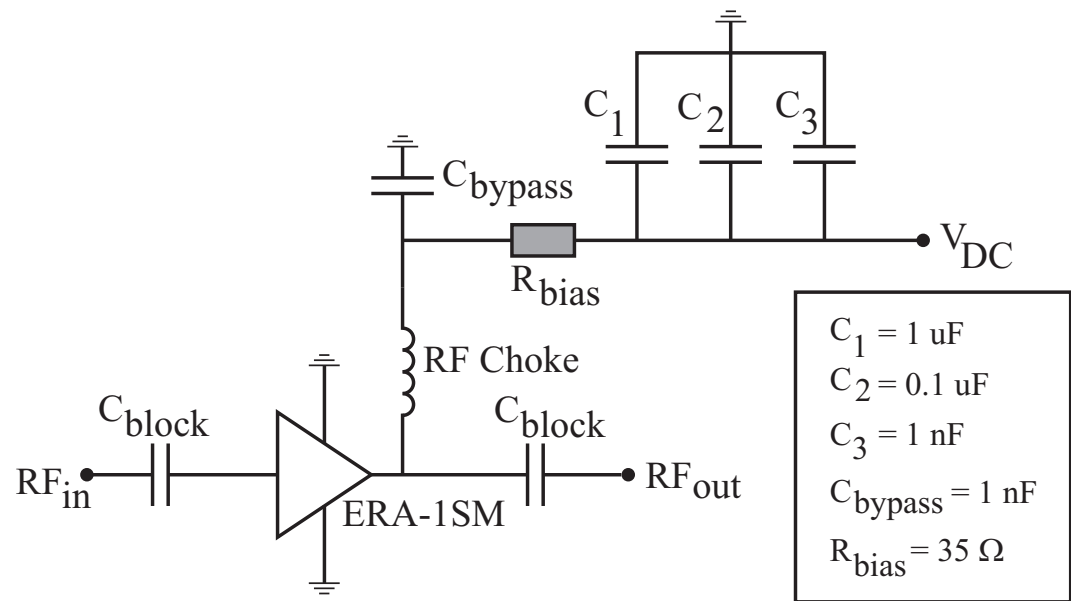

Figure 128: ERA-1SM RF amplifier biasing scheme. 


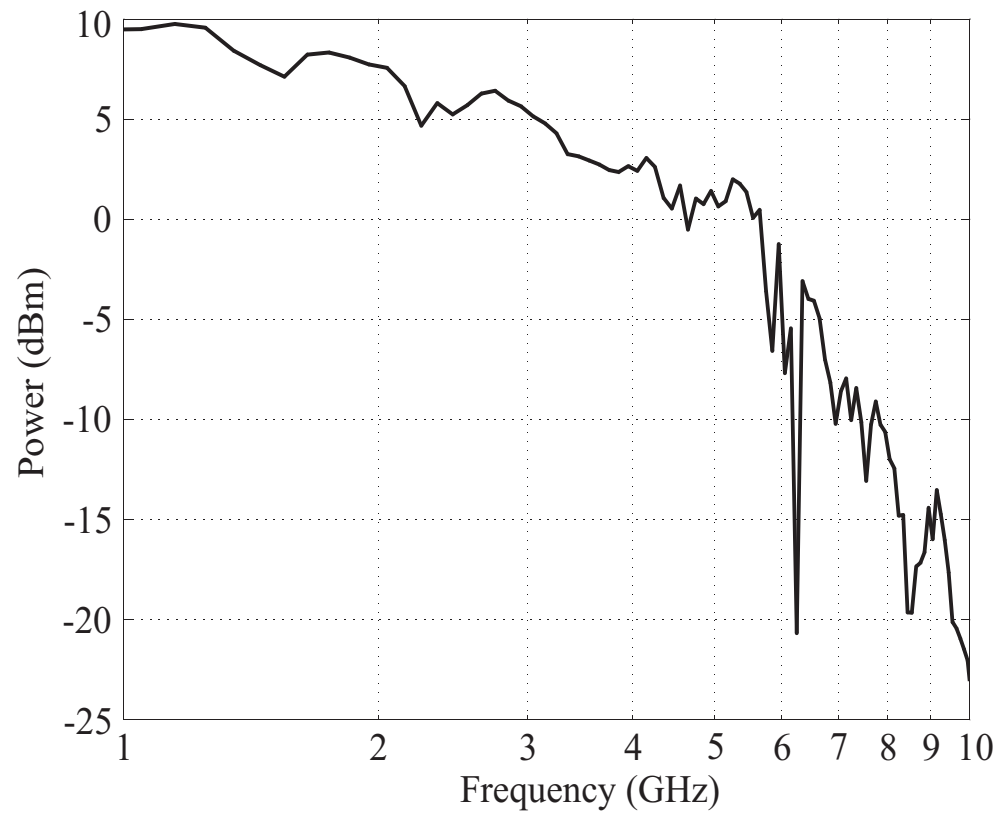

Figure 129: Measured output power from LR unit. 


\section{CHAPTER V}

\section{EXPERIMENTAL WORK}

This chapter details experimental work conducted to characterize the performance of the double-y balun. The performance of the double-y balun was evaluated via VSWR, insertion loss, and antenna pattern measurements. In addition, a sleeve balun (also known as the bazooka balun) was constructed and its performance evaluated via antenna pattern measurements. Pattern measurements were conducted for a $5 \mathrm{~cm}$ dipole, a $12 \mathrm{~cm}$ dipole, and a resistively loaded V-dipole. Antenna pattern measurements are compared with results obtained via NEC models.

\subsection{Measured S-Parameters of Double-Y Balun}

VSWR and insertion loss measurements were conducted for the double-y balun designed in Section 2.7. The balun transitions from a $50 \Omega$ coplanar waveguide (CPW) section to a $200 \Omega$ coplanar strip (CPS) section. Dimensions for the balun are illustrated in Fig. 69 in Section 2.7. Figure 130 illustrates two versions of the double-y balun over 25 mil FR4 substrate, one with 80 mil stubs and the other with 110 mil stubs. Figure 131 illustrates an expanded view of the double-y balun junction. The holes in the balun junction are through plated vias for the CPW bridges. A coaxial cable was directly soldered to the $50 \Omega$ coplanar waveguide (CPW) section of the double-y balun, as illustrated in Fig. 132. Figure 133 illustrates a bottom view of the double-y balun. CPW bridges were placed at the junction; additional CPW bridges were placed 230 mils apart. These bridges were connected to the CPW ground con-

ductors by vias. For VSWR measurements, the $200 \Omega$ CPS section was terminated with two $100 \Omega$ chip resistors in series (series resistors reduce parasitic capacitance 
at high frequencies), as illustrated in Fig. 134. Insertion loss measurements were conducted with back to back double-y baluns.

The HP8720D network analyzer was calibrated with $7 \mathrm{~mm}$ connectors, however, $3.5 \mathrm{~mm}$ SMA adaptors were used when conducting the actual measurements. Figure 135 illustrates the measured VSWR of the double-y balun with 80 mil stubs (with two additional CPW bridges) before and after time-gating the reflection from the adaptor. Time gating the reflection from the adaptor smoothes the VSWR data in the frequency domain. From Fig. 135, it is seen that the double-y balun with 80 mil stubs achieves a VSWR of less than 1.65 from 1 - 10 GHz. Figure 136 illustrates the time-domain reflected voltage of the double-y balun with 80 mil stubs when fed with a 1 V-peak input differentiated Gaussian pulse with spectral peak at $6 \mathrm{GHz}$. The largest reflection, after time-gating the reflection from the adaptor, is seen to occur at the coaxial line to CPW transition. The reflections at the balun junction are smaller than that from the load resistor, therefore, the balun junction is seen to be well matched. In addition, the junction seems to be properly transitioning between the CPW and CPS sections; the reflections at the junction are smaller than that from the matched load, therefore the smaller reflections at the balun junction when compared to the coaxial to CPW transition are not due to the balun loss.

Figure 137 illustrates the measured VSWR for the double-y balun with 110 mil stubs (with two additional CPW bridges as illustrated in Fig. 133). The balun achieves a VSWR of less than 1.65 from 1 - $10 \mathrm{GHz}$; VSWR results for the double-y balun with 80 and 110 mils agree very closely. Figure 138 illustrates the time-domain reflected voltage for the double-y balun with 110 mil stubs when fed with a $1 \mathrm{~V}$-peak input differentiated Gaussian pulse with spectral peak at $6 \mathrm{GHz}$. As was the case with the double-y balun with 80 mil stubs, the largest reflection occurs at the coaxial line to CPW transition. Reflections from the balun junction are smaller than the reflection from the load resistor, therefore, the balun junction is seen to be well matched. As 
was the case with the double-y balun with 80 mil stubs, the junction seems to be properly transitioning between the CPW and CPS sections, as the reflections at the junction are small.

The use of additional CPW bridges was investigated in Section 2.3.2, where it was shown that additional CPW bridges shift the CPW resonances to higher frequencies. $\mathrm{CPW}$ resonances occur when the $\mathrm{CPW}$ section of the balun is equal to multiples of a half wavelength. Figure 133 illustrates the double-y balun with additional CPW bridges. Measured $\mathrm{s}_{21}$ results for the double-y balun with and without additional CPW bridges are illustrated in Fig. 139, where it is seen that CPW resonances are suppressed via the use of additional CPW bridges.

From Fig. 140 it is seen that the double-y balun with 80 mil stubs exhibits an insertion loss of less than $1.8 \mathrm{~dB}$ from $800 \mathrm{MHz}$ to $10 \mathrm{GHz}$. The double-y balun with 110 mil stubs exhibits a higher insertion loss over the same frequency range; it achieves an insertion loss of less than $2.5 \mathrm{~dB}$ from $800 \mathrm{MHz}$ to $10 \mathrm{GHz}$. The double-y balun with 80 mil stubs is expected to exhibit lower insertion loss, since the resonance due to the length of the stubs occurs at a higher frequency. However, the overall performance of the baluns is dictated by the impedance tapers. Therefore, it is difficult to determine whether the $0.7 \mathrm{~dB}$ difference in insertion loss between the baluns is due to the length of the stubs or manufacturing tolerances.

Whether more than one additional CPW bridge is necessary to suppress the CPW resonance, was also investigated. Figure 141 illustrates the measured insertion loss for the double-y balun (80 mil stubs) without CPW bridges, with one additional CPW bridge (located 230 mils from CPW bridge at junction), and with two additional CPW bridges (placed 230 mils apart from each other as illustrated in Fig. 133). The measured results illustrate that additional CPW bridges do aid in the suppression of CPW resonances. 
The VSWR and insertion loss measurements describe the double-y balun as having a wide bandwidth of operation. However, these parameters provide little insight into the degree of balancing achieved by the balun when used for feeding an antenna. Therefore, to characterize the double-y balun more thoroughly, antenna pattern measurements are conducted with the balun feeding a dipole and a resistively loaded V-dipole.

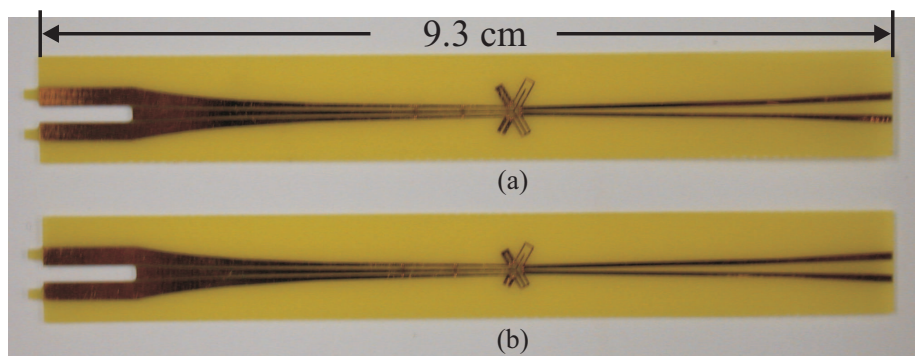

Figure 130: Illustration of double-y balun designed with (a) 110 mil stubs and (b) 80 mil stubs.

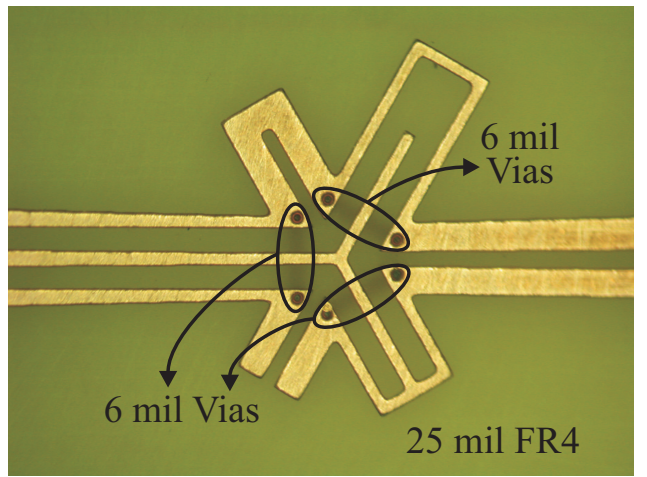

Figure 131: Expanded view of double-y balun junction illustrating through plated via holes for CPW bridges. 


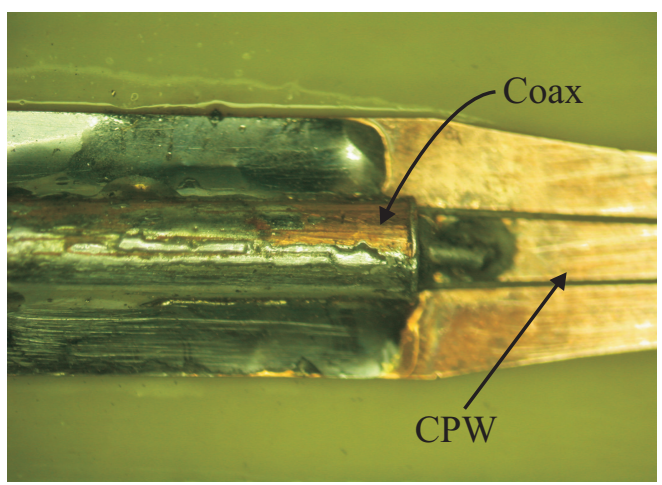

Figure 132: Expanded view of coaxial line soldered to the $50 \Omega \mathrm{CPW}$ section.

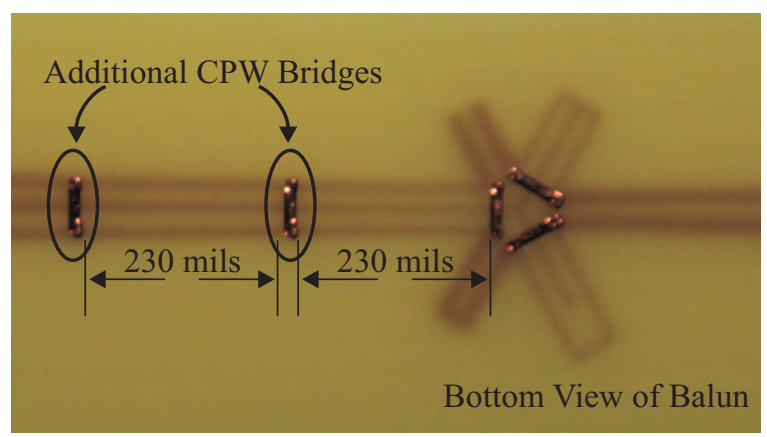

Figure 133: Bottom view of double-y balun with additional CPW bridges.

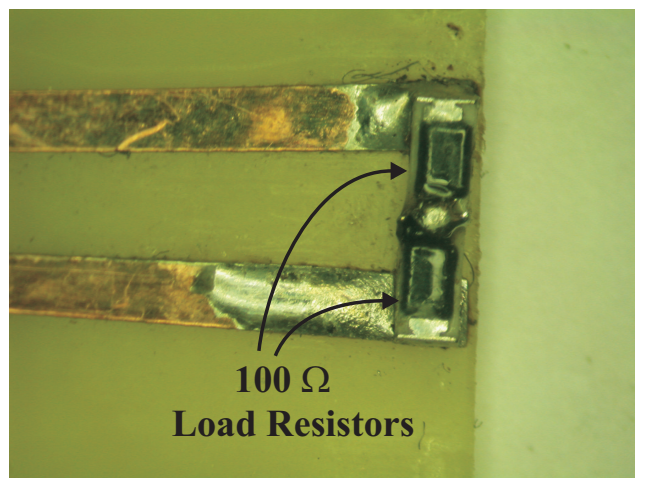

Figure 134: Expanded view of two $100 \Omega$ series load resistors soldered to the CPS section for VSWR measurement. 


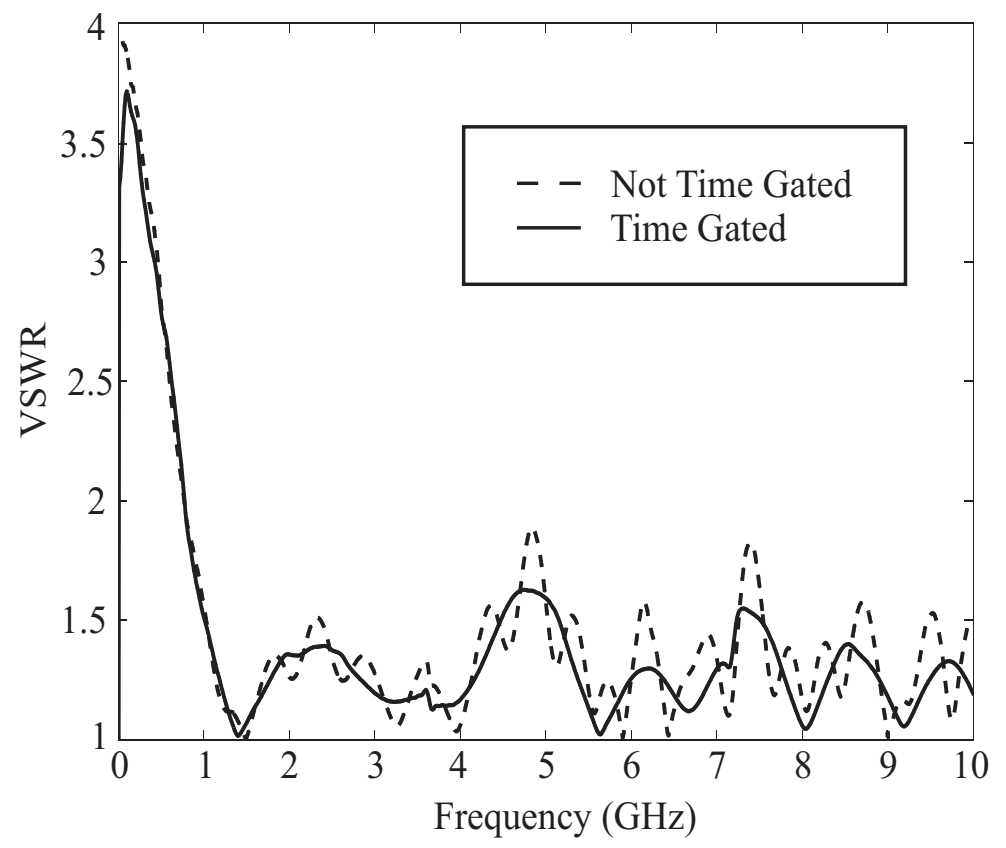

Figure 135: Measured VSWR of double-y balun with 80 mil stubs and $200 \Omega$ load resistor.

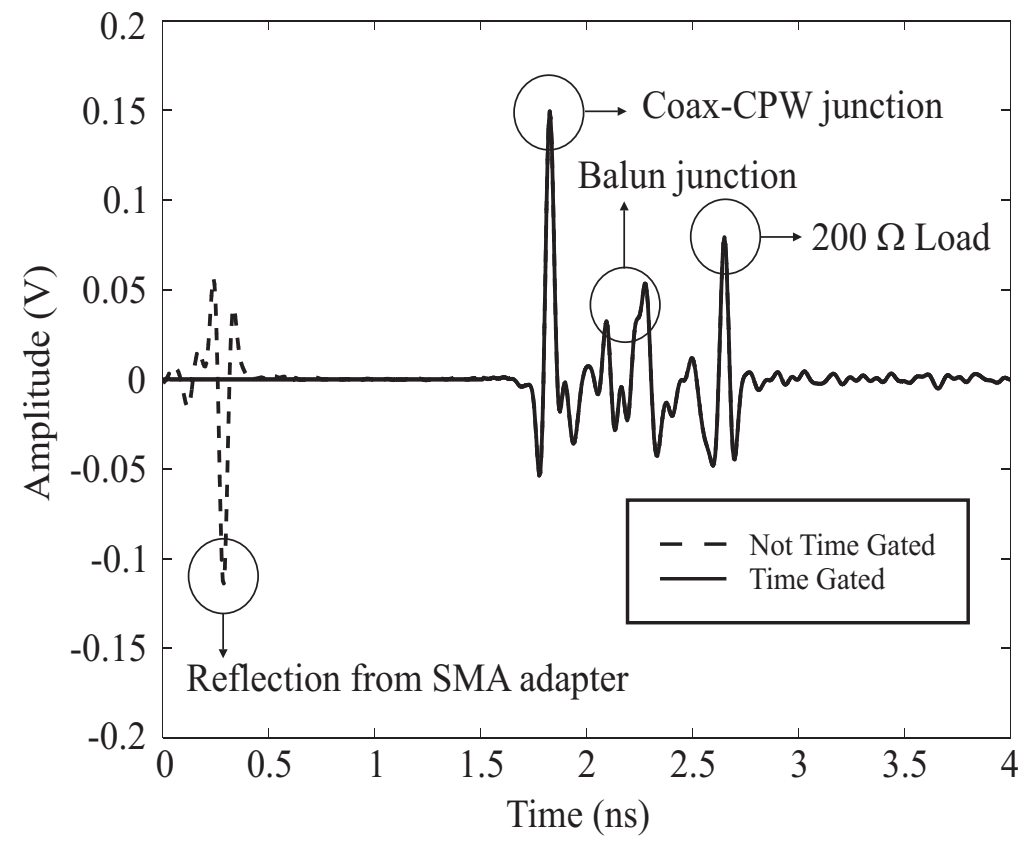

Figure 136: Time-domain reflected voltage of the double-y balun with 80 mil stubs when fed with a $1 \mathrm{~V}$-peak input differentiated Gaussian pulse with spectral peak at $6 \mathrm{GHz}$. 


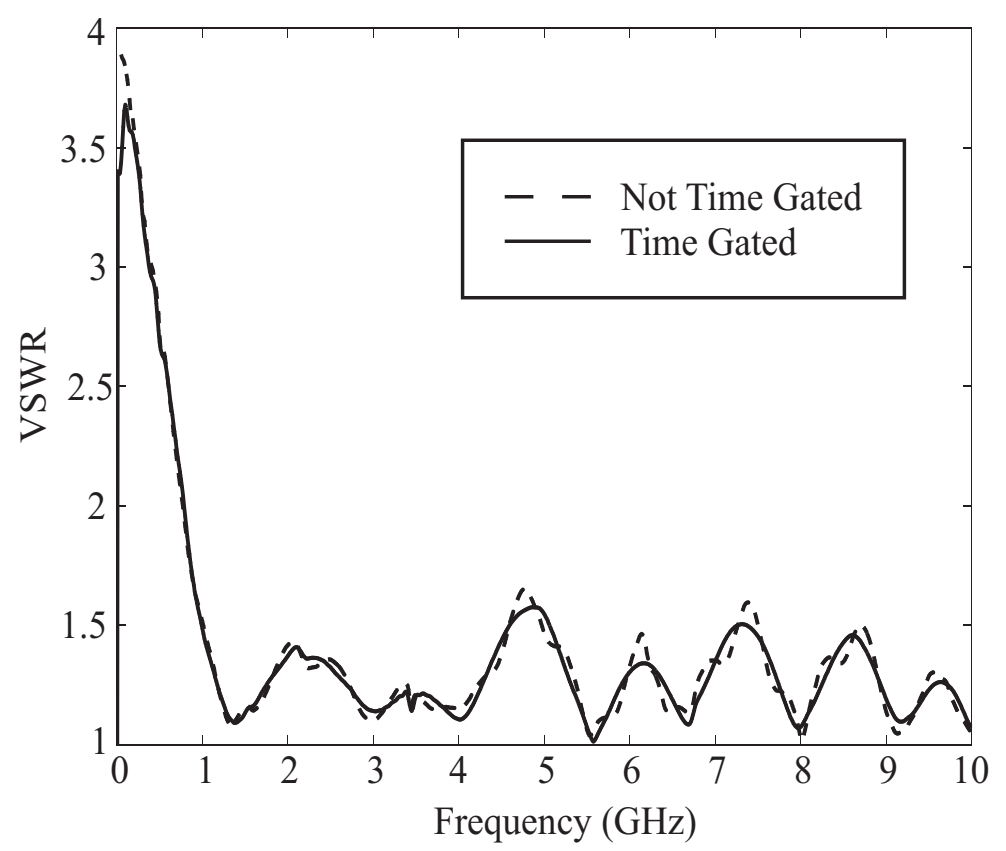

Figure 137: Measured VSWR of double-y balun with 110 mil stubs and $200 \Omega$ load resistor.

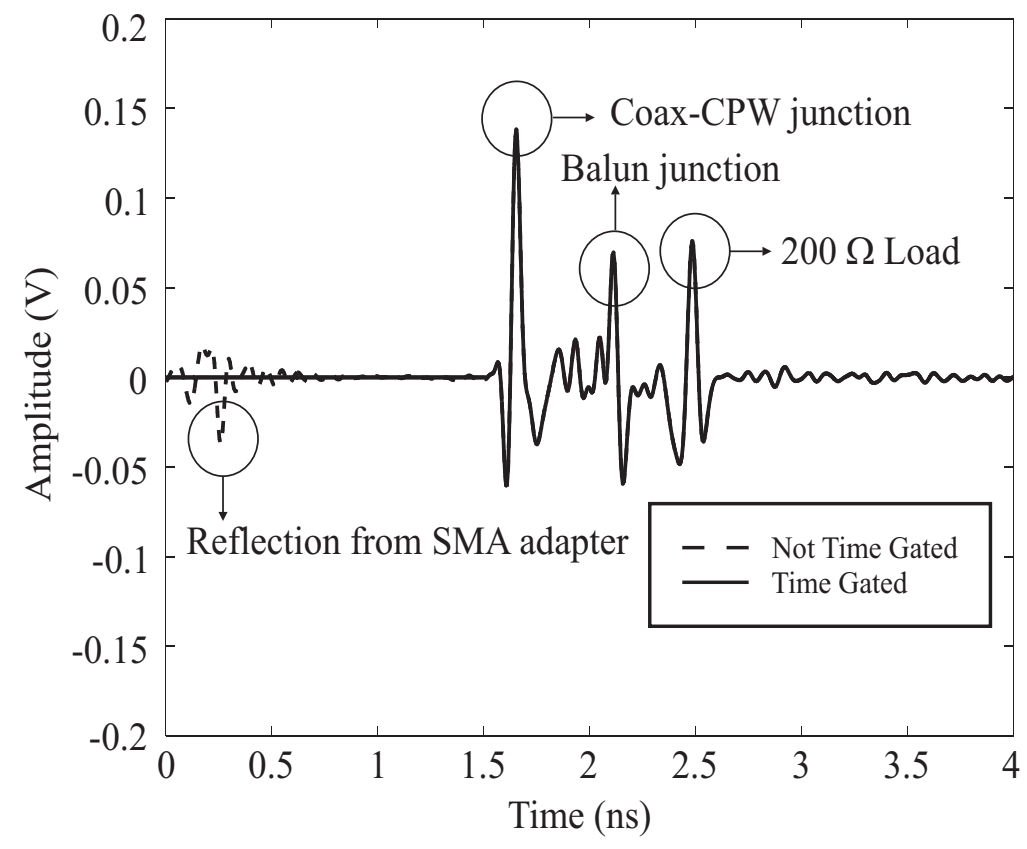

Figure 138: Time-domain reflected voltage of the double-y balun with 110 mil stubs when fed with a $1 \mathrm{~V}$-peak input differentiated Gaussian pulse with spectral peak at $6 \mathrm{GHz}$. 


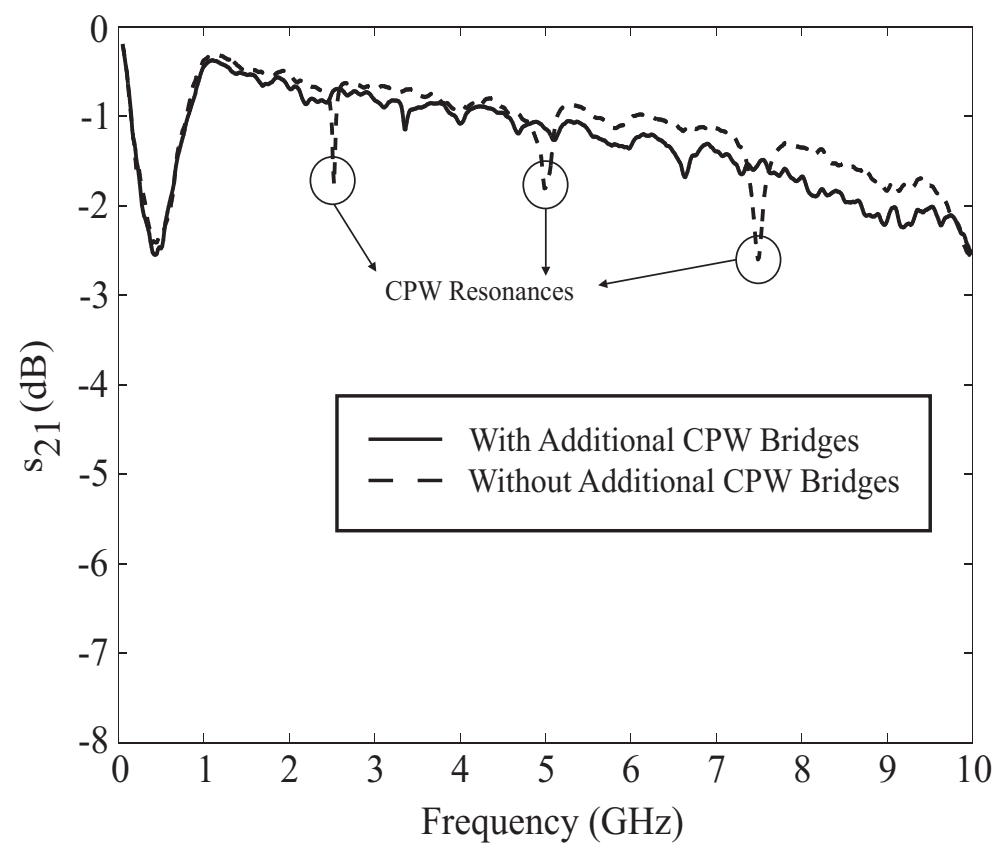

Figure 139: Measured insertion loss of double-y balun with 110 mil stubs with and without additional CPW bridges.

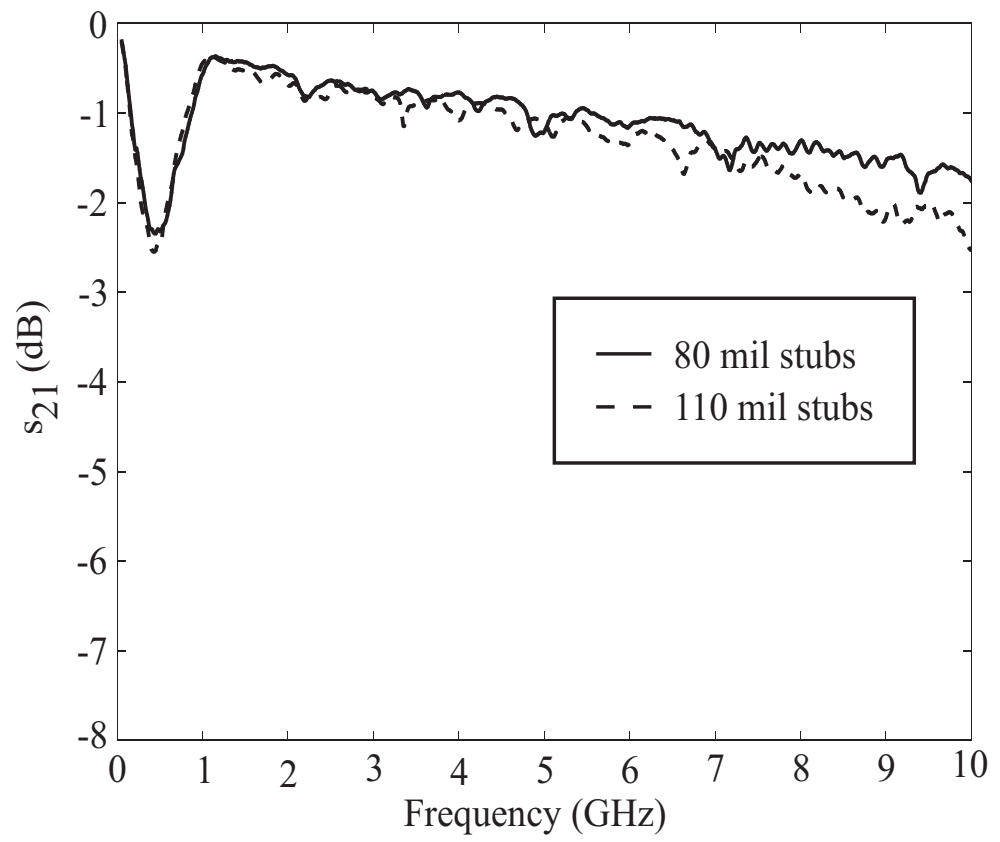

Figure 140: Measured insertion loss of double-y balun with 80 and 110 mil stubs. 


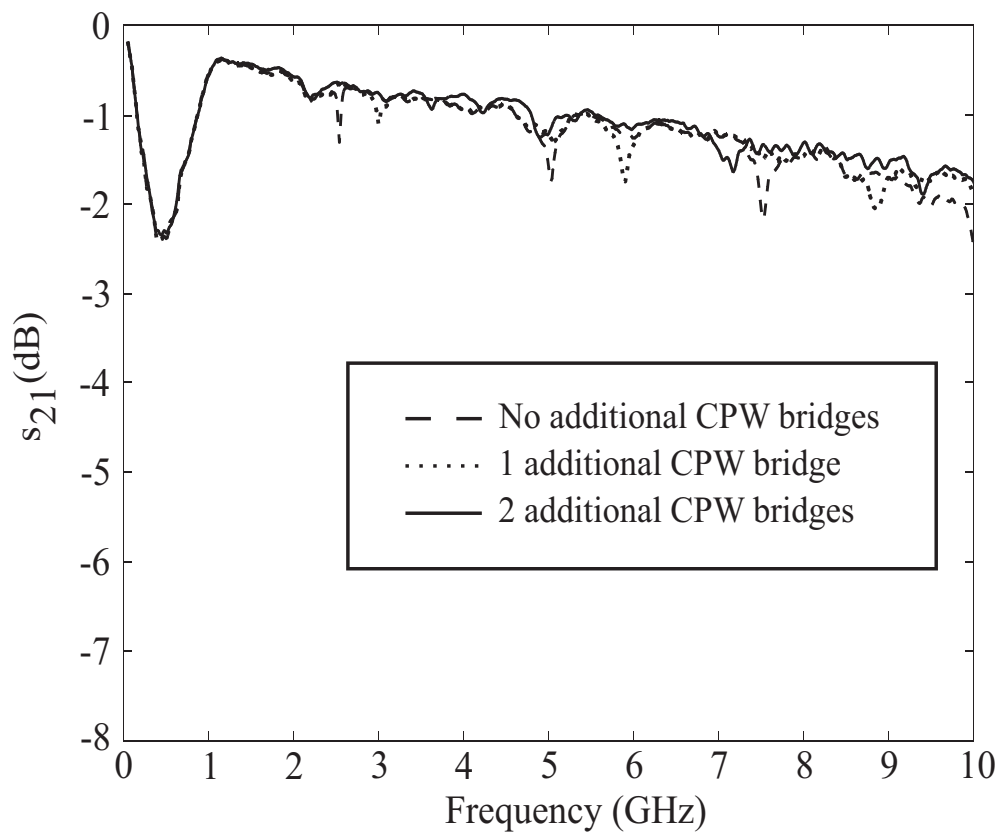

Figure 141: Measured insertion loss of double-y balun (80 mil stubs) without additional CPW bridges, one additional CPW bridge, and two additional CPW bridges. 


\subsection{Antenna Pattern Measurements}

Figure 142 illustrates a block diagram of the measurement system constructed for conducting antenna pattern measurements. The antenna under test (AUT) was mounted on a Velmex rotary positioner, which was controlled using a Velmex VP9000 motor controller. The motor controller was connected serially to a PC using the RS-232 port. The $8720 \mathrm{D}$ HP network analyzer was also connected to the PC using a GPIB interface. An existing LabVIEW (graphical programming language that enables a PC to control external devices) program was modified to control the positioner using the serial RS-232 interface. The entire measurement system was automated using the PC.

To obtain accurate antenna pattern measurements, the AUT has to be aligned properly with the center of the positioner as well the probe antenna. As illustrated in Fig. 143, the AUT was aligned with the center of the positioner as well as the reference probe antenna by using a rotary laser leveler. Aligning the AUT with the positioner at two points ensured that the AUT would rotate properly about the center of the positioner. Figure 144 illustrates the AUT on the rotary position.

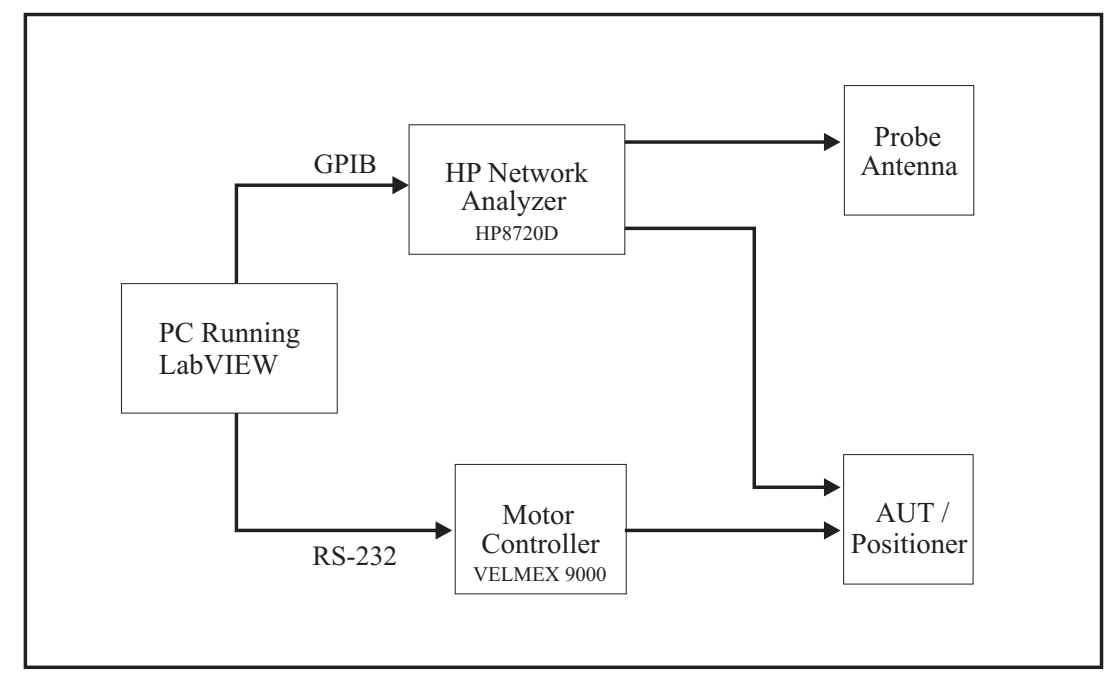

Figure 142: Block diagram illustrating measurement setup constructed for conducting antenna pattern measurements. 


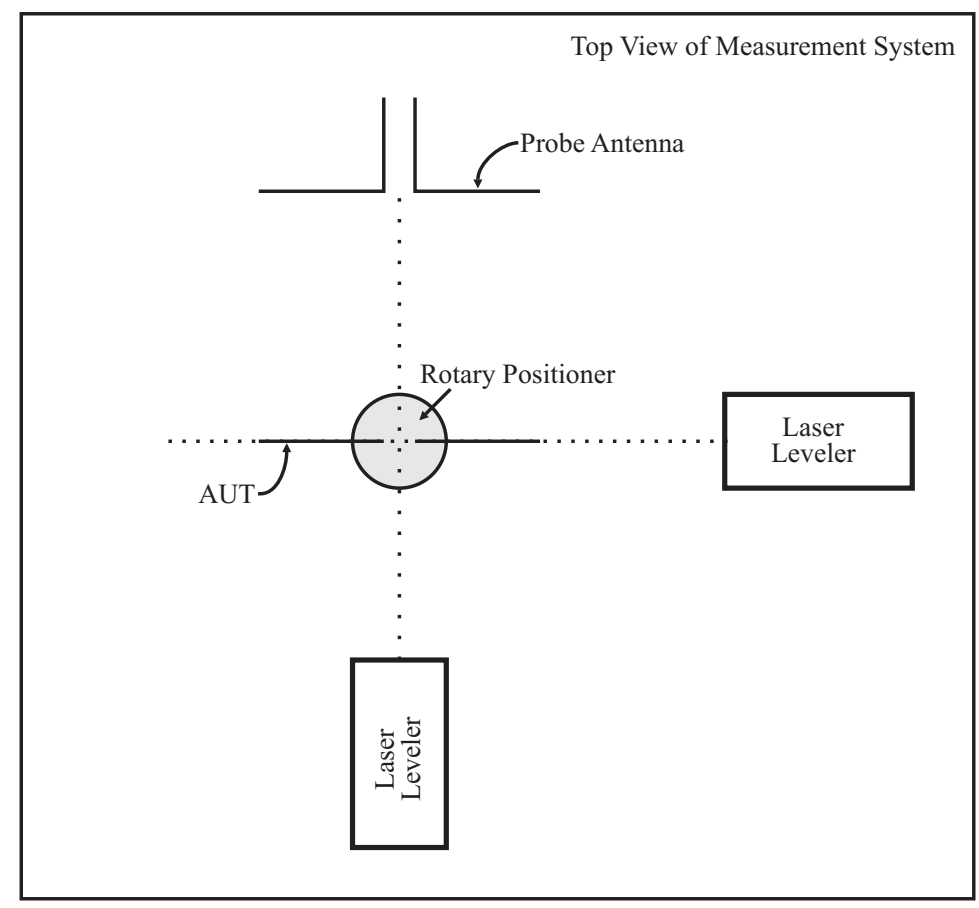

Figure 143: Alignment of AUT with rotary positioner and reference probe.

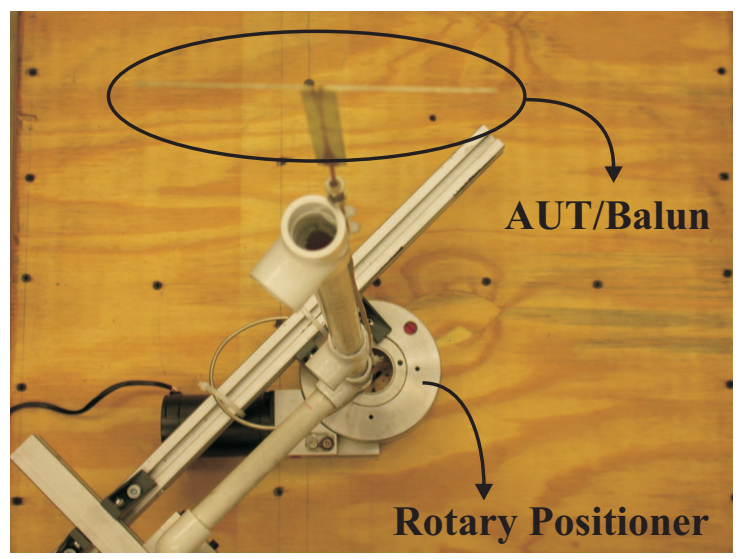

Figure 144: Illustration of AUT on rotary positioner.

\subsection{1 $5 \mathrm{~cm}$ Dipole Azimuth Patterns Without Optical Link}

Amplitude pattern measurements were conducted along the azimuth plane (defined Fig. 84) for a $5 \mathrm{~cm}$ dipole fed without a balun, with the double-y balun, and with a sleeve balun. The AUT was attached to the positioner as illustrated in Fig. 145a. The positioner was covered with microwave absorber to minimize reflections. The probe antenna, illustrated in Fig. 145b, was fed with the double-y balun and embedded in 
microwave absorber. Coaxial lines from the network analyzer were connected to the probe antenna and the AUT.

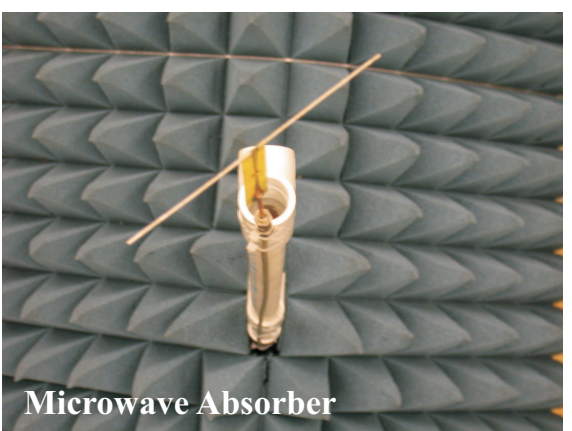

(a)

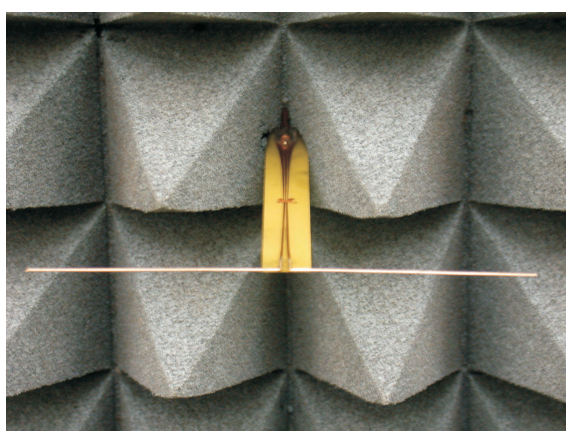

(b)

Figure 145: Illustration of (a) AUT on rotary positioner covered with microwave absorber and (b) probe antenna embedded in microwave absorber.

Measured azimuth patterns at $\lambda / 2$ and $3 \lambda / 2$ are illustrated in Fig. 147 for a $5 \mathrm{~cm}$ unbalanced dipole. The unbalanced dipole was constructed by extending the center conductor of the coaxial line to form one arm of the dipole. The other arm of the dipole was soldered to the outer conductor of the coaxial line, as illustrated in Fig. 146. As seen in Fig. 147, the measured amplitude patterns at $\lambda / 2$ and $3 \lambda / 2$ agree very closely with results obtained using the NEC model in Fig. 76 with $\alpha=0.1$. Figure 148 illustrates the time-domain pattern of the measured voltage at the probe with an incident double differentiated Gaussian pulse (with spectral peak at $3 \mathrm{GHz}$ ) transmitted by the dipole fed without a balun. The time-domain pattern serves to illustrate the directional pattern of the antenna. It is seen that the dipole radiates strongly in the broadside direction $\left(\phi=90^{\circ}\right.$ and $\left.270^{\circ}\right)$, while little or nothing is radiated along the axis of its arms $\left(\phi=0^{\circ}\right.$ and $\left.180^{\circ}\right)$.

The asymmetry in the pattern of the $5 \mathrm{~cm}$ unbalanced dipole was illustrated in Fig. 147b. Asymmetry in the pattern is observed because of the deep nulls near $70^{\circ}$ and $310^{\circ}$. Next, this asymmetry is investigated in the time domain. Figure 149 compares the time-domain plots from Fig. 154 at $70^{\circ}$ and $110^{\circ}$. It is interesting to observe that both waveforms agree very closely. Although the dipole pattern is asymmetrical at 
$3 \lambda / 2$, the effects are not observed in the time domain. This is because the patterns in Fig. 147 are normalized patterns. The dipole actually radiates stronger at $\lambda / 2$ than at $3 \lambda / 2$. Therefore, the time-domain response of the dipole is more dependant on the behavior of the dipole at $\lambda / 2$ than at $3 \lambda / 2$. There is little difference in the time-domain plot in Fig. 149, and correspondingly there is little asymmetry in the patterns at $\lambda / 2$ in Fig. 147a.

Measured azimuth patterns for a $5 \mathrm{~cm}$ dipole fed with the double-y balun are illustrated in Fig. 150. It is seen that the measured patterns agree very closely with patterns generated for a balanced dipole using the NEC model in Fig. 76 with $\alpha=0.5$. At $\lambda / 2$ and $3 \lambda / 2$, the dipole fed with the double-y balun shows symmetry in the measured amplitude patterns in all four quadrants. In contrast, the $\lambda / 2$ pattern for the dipole fed without a balun is slightly rotated. The $3 \lambda / 2$ pattern for the dipole fed without a balun has a deep null between $60^{\circ}$ and $90^{\circ}$ and between $270^{\circ}$ and $300^{\circ}$. The time-domain pattern is illustrated in Fig. 151. As was the case with the unbalanced dipole, the dipole radiates strongly along the broadside direction.

Amplitude patterns were also measured for a $5 \mathrm{~cm}$ dipole fed with a sleeve balun (discussed in Chapter 1). Figure 152 illustrates the dipole fed with the sleeve balun. The normalized amplitude patterns at $\lambda / 2$ and $3 \lambda / 2$ are illustrated in Fig. 153. It is seen that the amplitude pattern at $\lambda / 2$ agrees closely with the pattern computed via NEC for a balanced dipole. However, the pattern at $3 \lambda / 2$ does not agree as well with the NEC pattern for a balanced dipole. Although we observe symmetry in the resulting pattern, there is some discrepancy between the measured results and that obtained from the NEC model. Figure 154 illustrates the time-domain pattern for the dipole fed with the sleeve balun. Maximum radiation is seen to occur along the broadside direction and minimum radiation is seen to occur along the dipole arms.

Figure 155 compares the time domain signal at $\phi=90^{\circ}$ received at the probe with an unbalanced dipole, a dipole fed with the double-y balun, and a dipole fed with a 


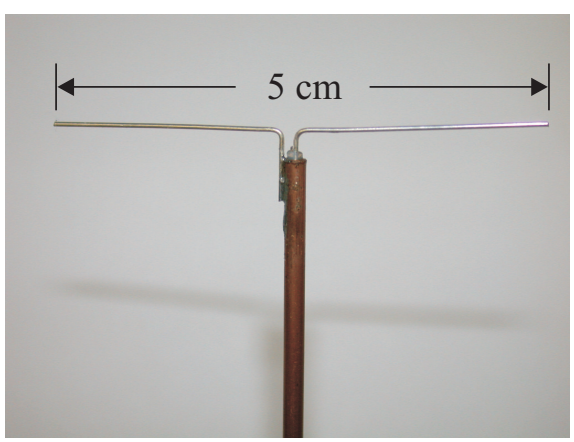

Figure 146: Illustration of $5 \mathrm{~cm}$ dipole fed directly with a coaxial line, without a balun.

sleeve balun transmitting an incident double differentiated Gaussian pulse. To remove clutter from the measurement environment, data beyond 9.5 ns was time-gated out. The total time window of 3.5 ns was dictated by the rotary positioner structure (nearest object from which reflections could clutter the data). Time-gating feedline reflections should not affect the resulting patterns significantly along the azimuth plane, as detailed in Section 3.5.1. 


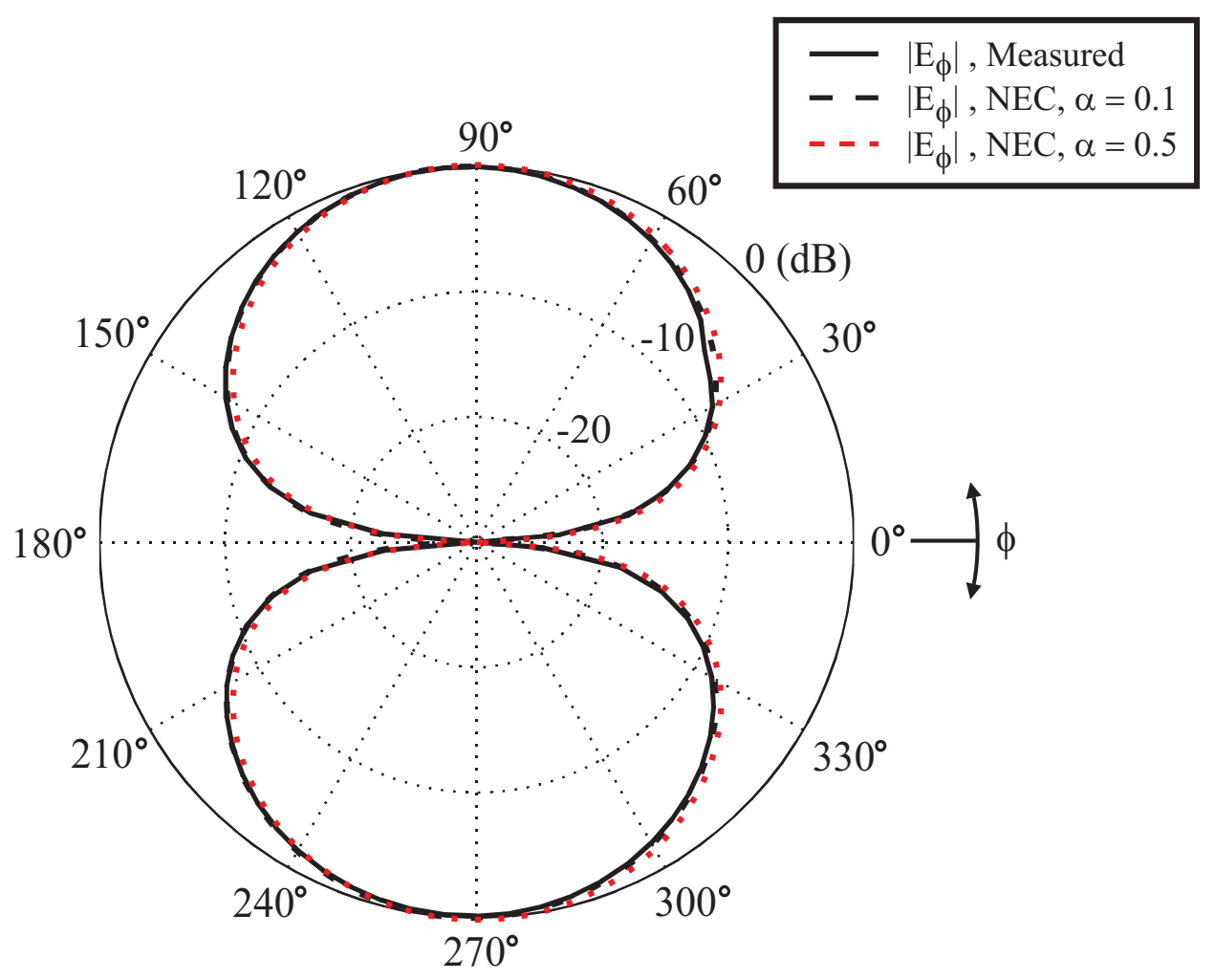

(a)

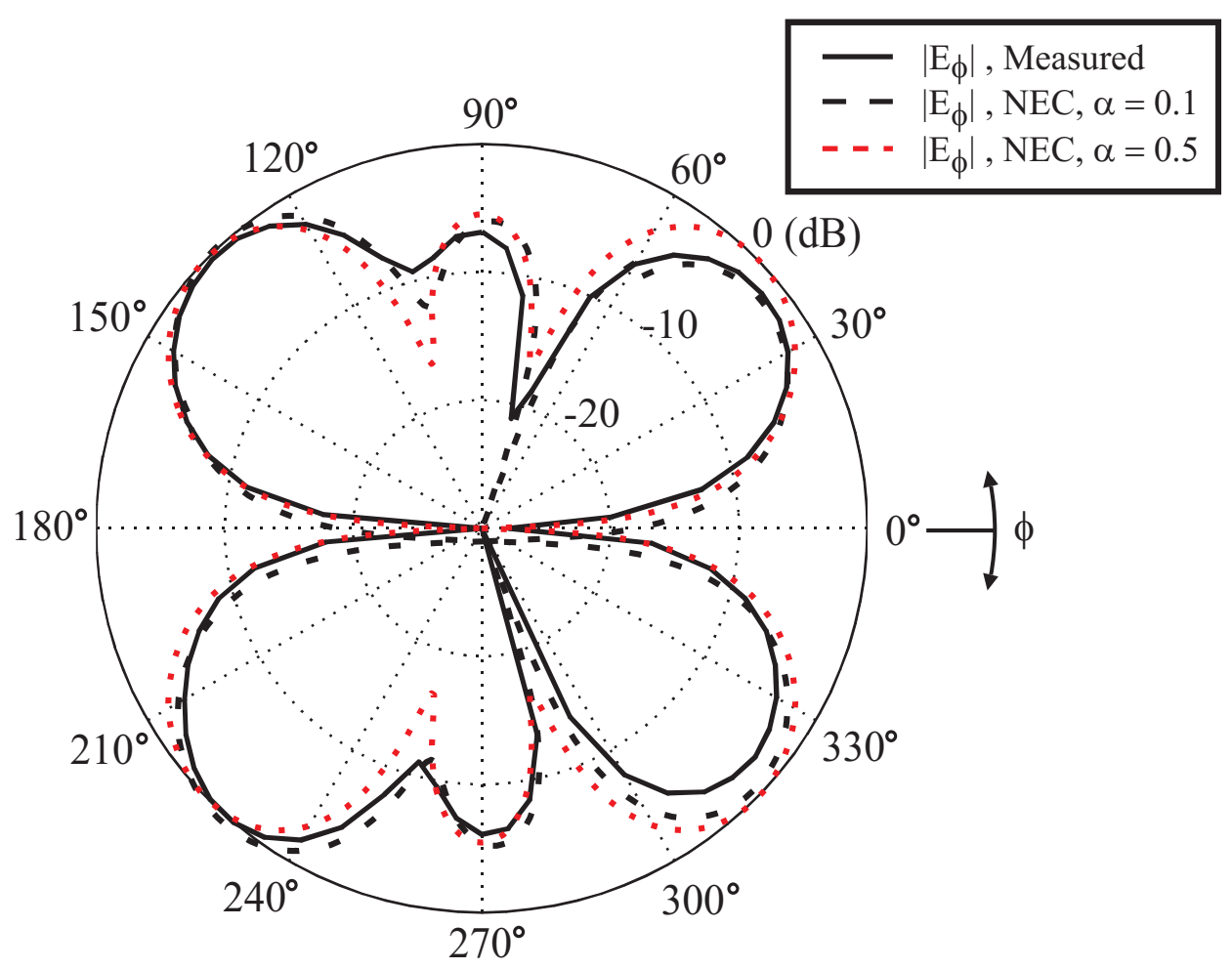

(b)

Figure 147: Measured vs. NEC (NEC model in Fig. 76 with $\alpha=0.1$ for unbalanced and $\alpha=0.5$ for balanced dipole) azimuth amplitude patterns for unbalanced $5 \mathrm{~cm}$ dipole at (a) $\lambda / 2$ and (b) $3 \lambda / 2$. 


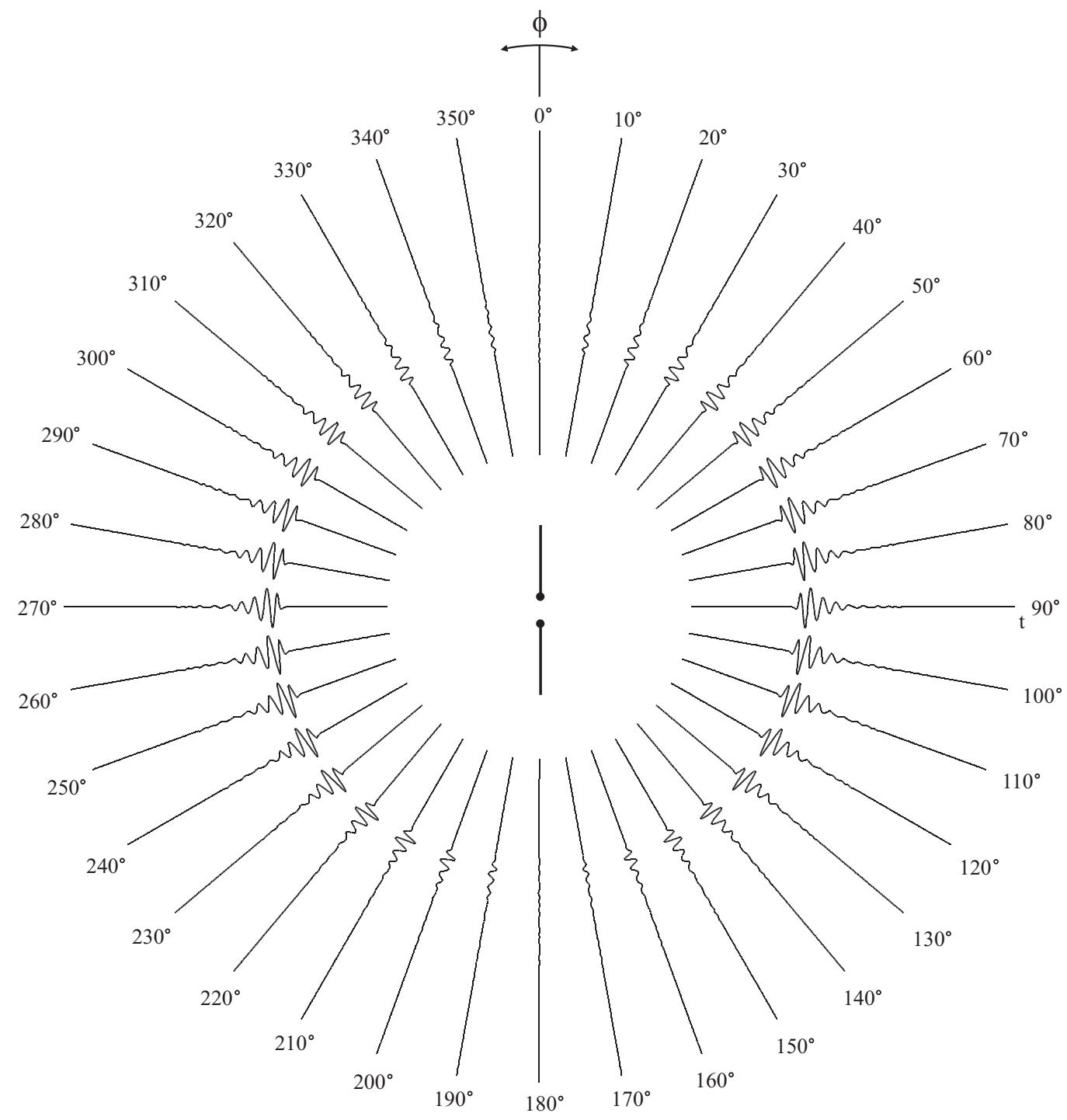

Figure 148: Time-domain pattern of the measured voltage at the probe with an incident double differentiated Gaussian pulse (spectral peak at $3 \mathrm{GHz}$ ) transmitted by a $5 \mathrm{~cm}$ dipole fed without a balun. 


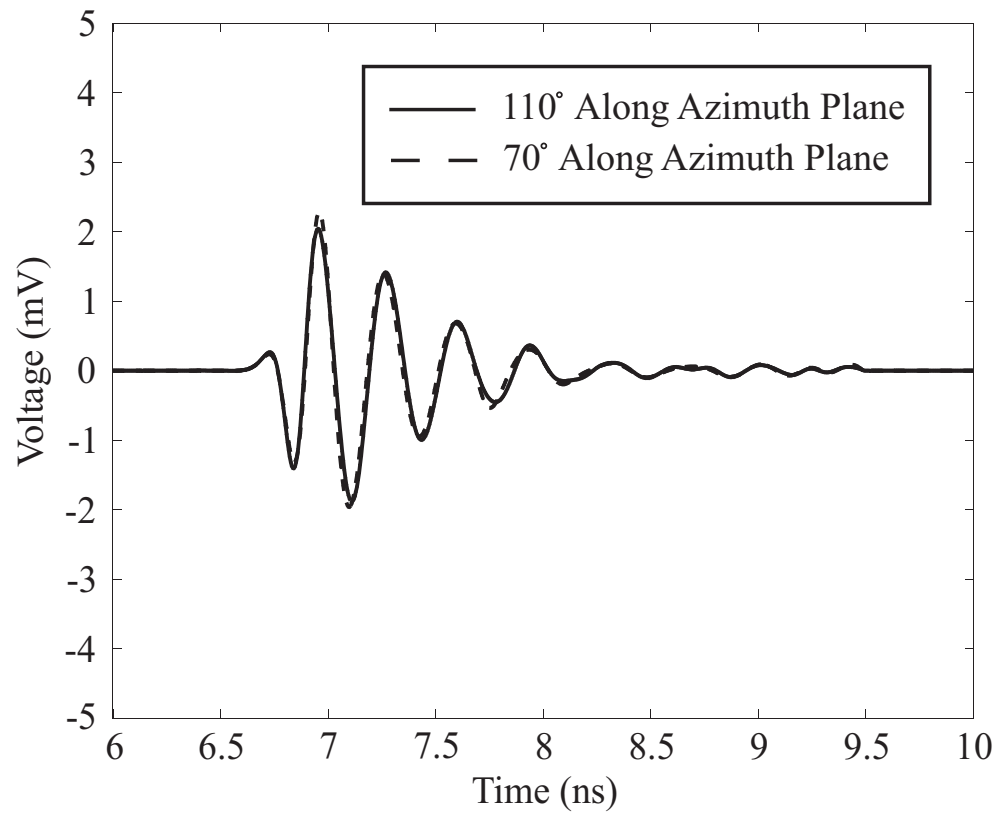

Figure 149: Plot comparing time-domain waveforms from Fig. 148 at $70^{\circ}$ and $110^{\circ}$. 


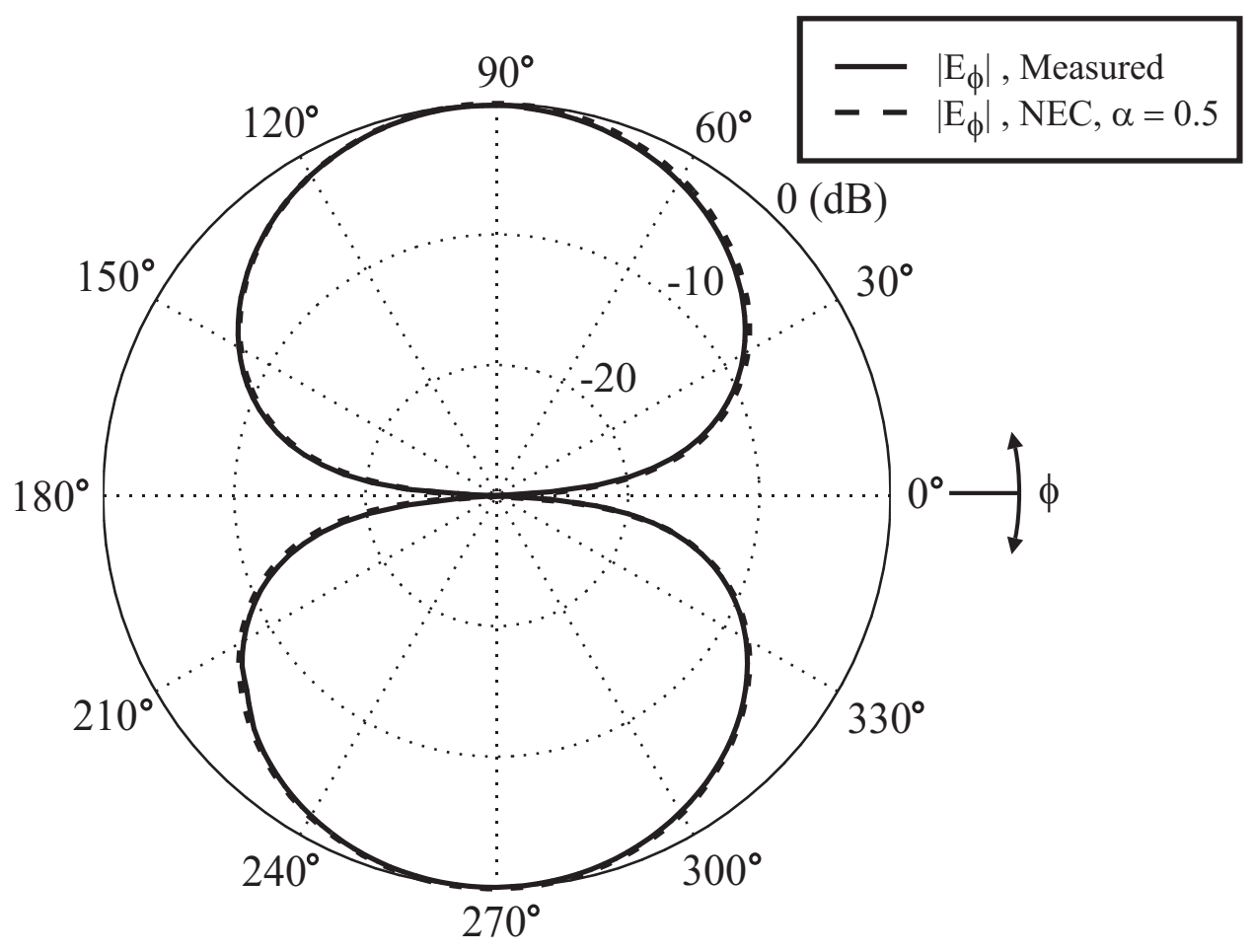

(a)

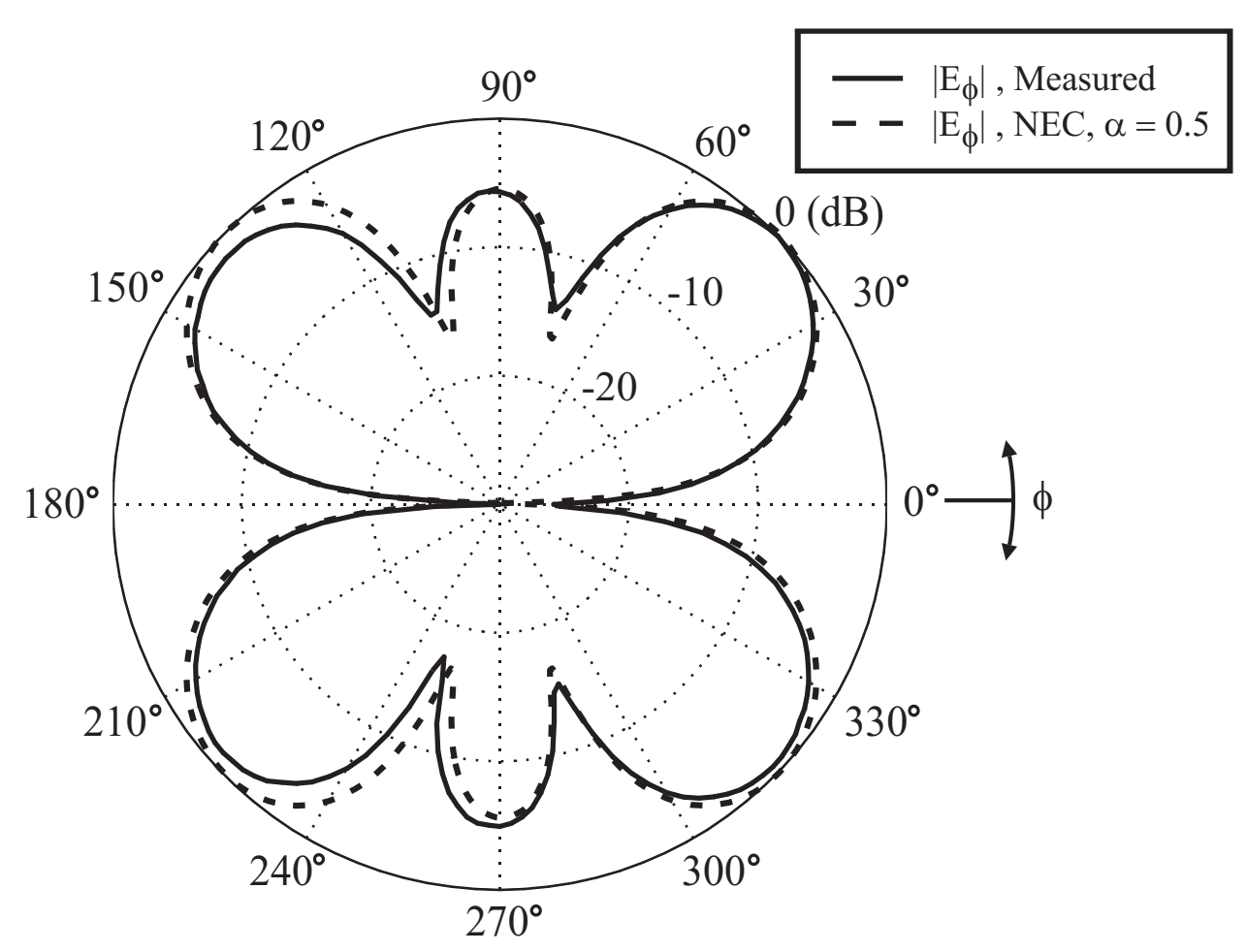

(b)

Figure 150: Measured vs. NEC (NEC model in Fig. 76 with $\alpha=0.5$ for balanced dipole) azimuth amplitude patterns for $5 \mathrm{~cm}$ dipole fed with double-y balun at (a) $\lambda / 2$ and (b) $3 \lambda / 2$. 


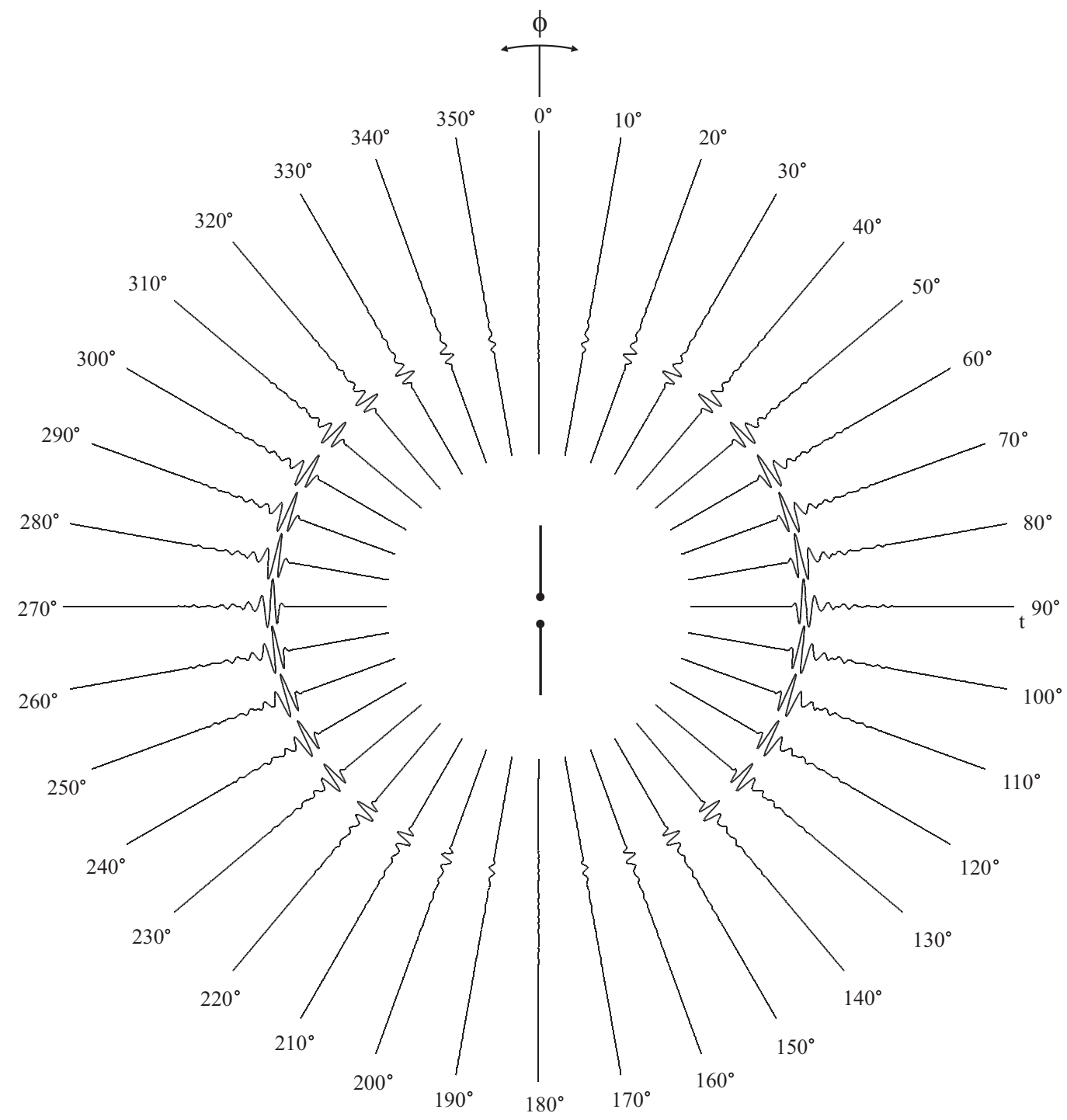

Figure 151: Time-domain pattern of the measured voltage at the probe with an incident double differentiated Gaussian pulse (spectral peak at $3 \mathrm{GHz}$ ) transmitted by a $5 \mathrm{~cm}$ dipole fed with the double-y balun.

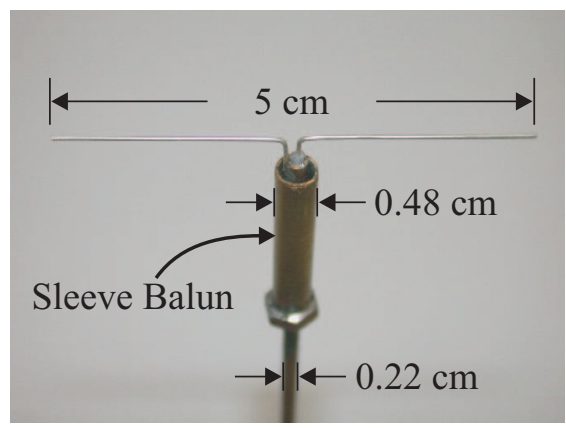

Figure 152: Illustration of $5 \mathrm{~cm}$ dipole fed with sleeve balun. 


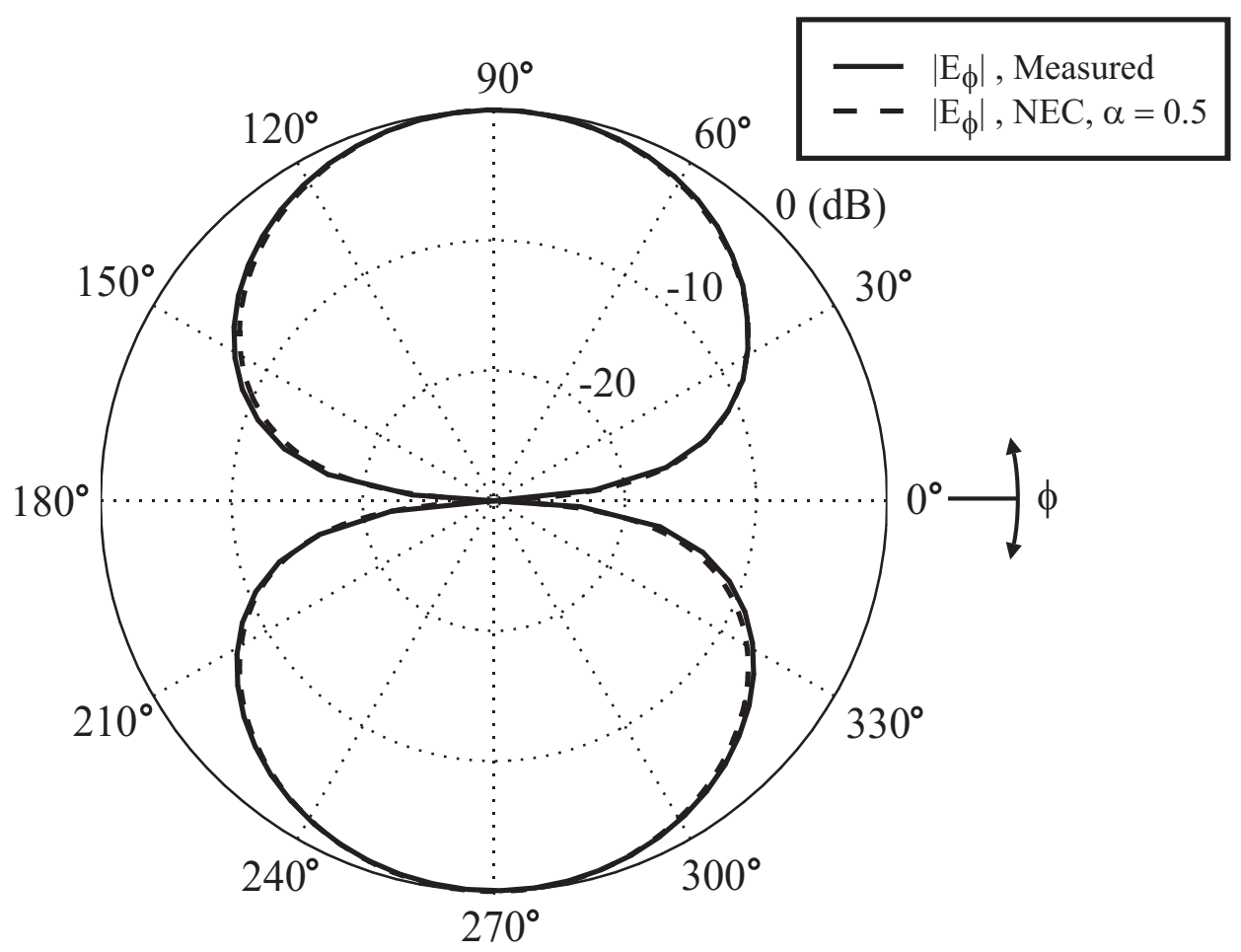

(a)

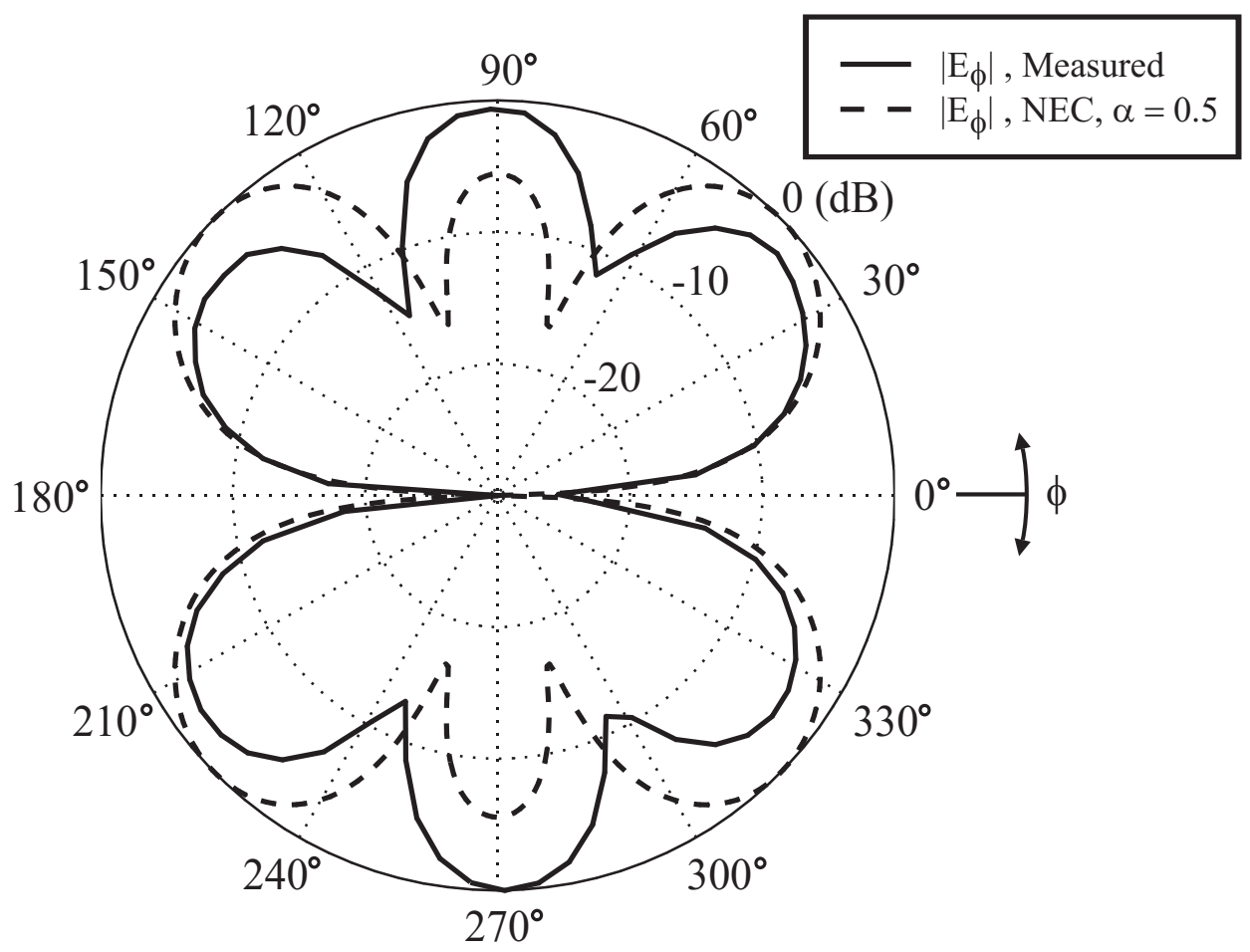

(b)

Figure 153: Measured vs. NEC (NEC model in Fig. 76 with $\alpha=0.5$ for balanced dipole) azimuth amplitude patterns for $5 \mathrm{~cm}$ dipole fed with sleeve balun at (a) $\lambda / 2$ and (b) $3 \lambda / 2$. 


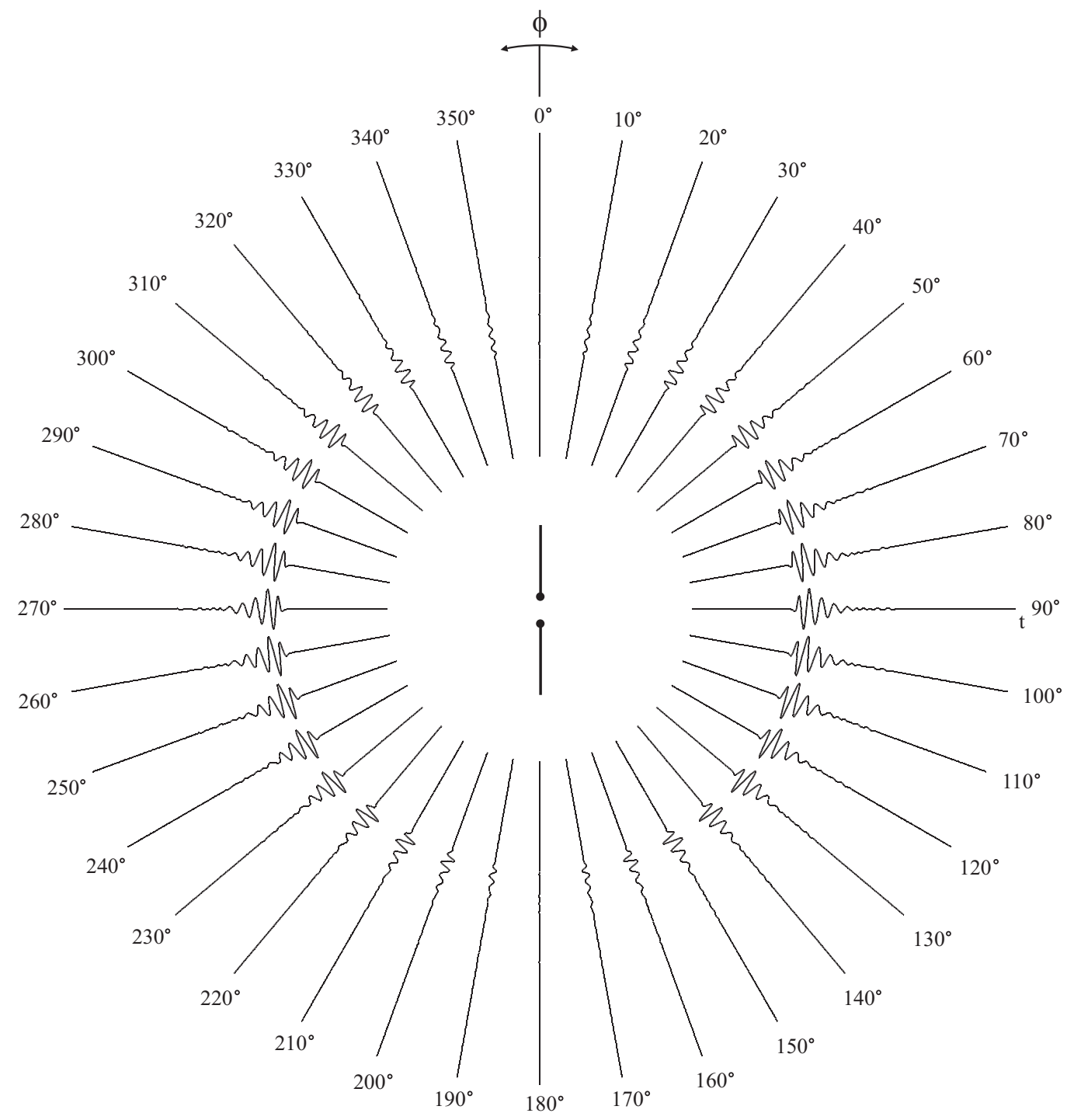

Figure 154: Time-domain pattern of the measured voltage at the probe with an incident double differentiated Gaussian pulse (spectral peak at $3 \mathrm{GHz}$ ) transmitted by a $5 \mathrm{~cm}$ dipole fed with the sleeve balun. 


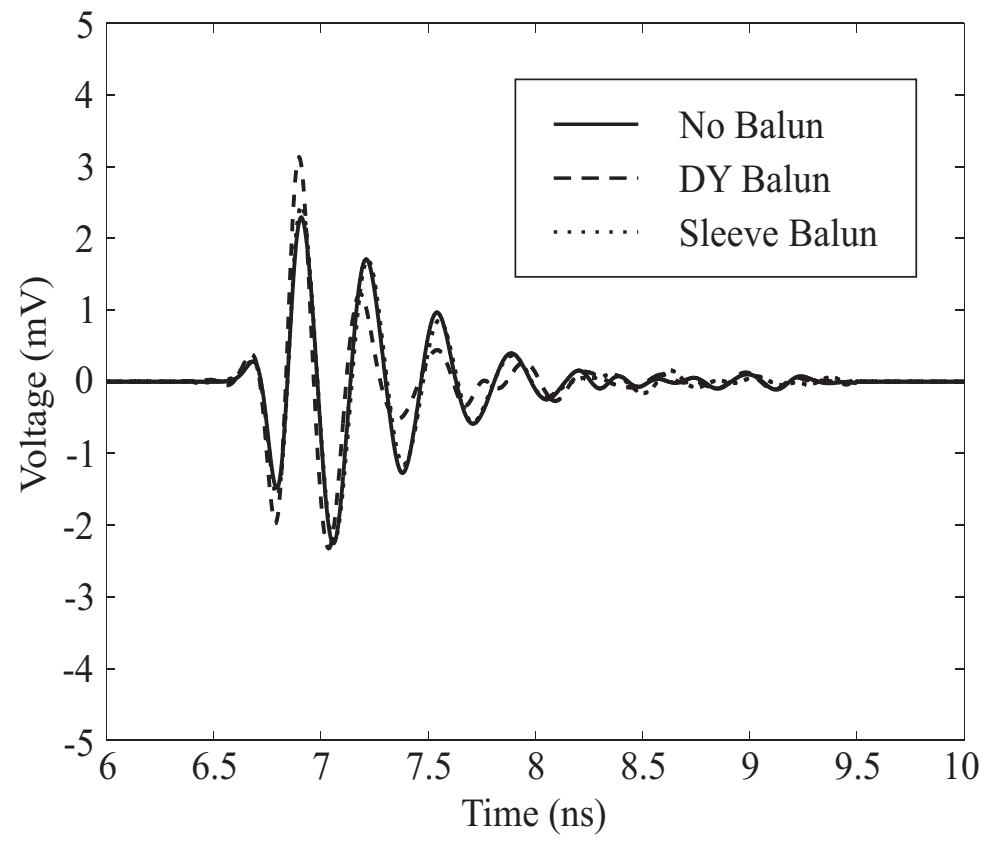

Figure 155: Time-domain plot of the measured voltage at the probe with an incident double differentiated Gaussian pulse (spectral peak at $3 \mathrm{GHz}$ ) transmitted by a $5 \mathrm{~cm}$ dipole along $\phi=0^{\circ}$. 


\subsection{2 $12 \mathrm{~cm}$ Dipole Patterns Without Optical Link}

Amplitude pattern measurements along the azimuth plane were conducted for a 12 cm dipole fed with and without the double-y balun. Measured results are compared with results obtained using the NEC model in Fig. 76 (with $\alpha=0.5$ for a balanced dipole and $\alpha=0.1$ for an unbalanced dipole). The $12 \mathrm{~cm}$ dipole allows measured amplitude patterns to be analyzed at $\lambda / 2,3 \lambda / 2$, and $5 \lambda / 2$. The $12 \mathrm{~cm}$ unbalanced dipole was constructed similarly to the $5 \mathrm{~cm}$ unbalanced dipole, and is illustrated in Fig. 156; the center conductor of the coaxial line was extended out to form one arm of the dipole. The other arm of the dipole was soldered to the outer conductor of the coaxial line. The measurement setup was the same as that used for pattern measurements with the $5 \mathrm{~cm}$ dipole in Section 5.2.1.

Figure 157a compares the measured normalized amplitude pattern of the unbalanced $12 \mathrm{~cm}$ dipole with the amplitude pattern obtained via NEC for an unbalanced dipole. Both patterns agree closely, however, as stated in previous sections, it is difficult to discern between amplitude patterns along the azimuth plane for a balanced vs. unbalanced dipole at $\lambda / 2$. The overall shape of the pattern remains the same at $\lambda / 2$, regardless of whether the feed is balanced or unbalanced.

The effects of an unbalanced feed are seen more clearly at $3 \lambda / 2$, as illustrated in Fig. 157b. The measured pattern for the unbalanced dipole agrees very well with the pattern obtained via the NEC model (Fig. 76 with $\alpha=0.1$ ) for an unbalanced dipole at $3 \lambda / 2$. The pattern exhibits a deep null between $60^{\circ}$ and $90^{\circ}$ and between $270^{\circ}$ and $300^{\circ}$. These deep nulls should not be present in the pattern of a balanced dipole at $3 \lambda / 2$, as illustrated by NEC results in Fig. $85 \mathrm{~b}$.

The measured pattern at $5 \lambda / 2$, illustrated in Fig. $157 \mathrm{c}$, also agrees very well with NEC results obtained for an unbalanced dipole at $5 \lambda / 2$. Again, for a balanced dipole, the pattern should be symmetrical about all four quadrants, as obtained via NEC and illustrated in Fig. 85c. While the pattern exhibits some symmetry between 


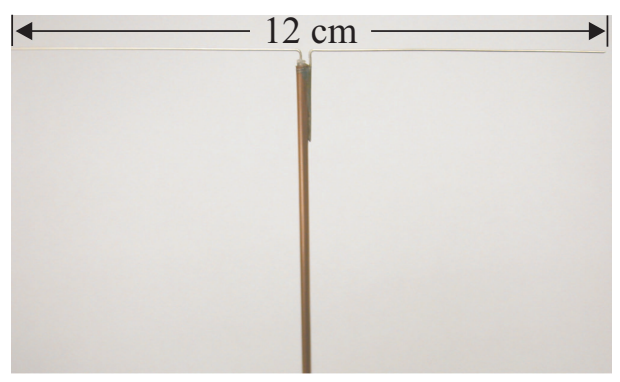

Figure 156: Illustration of $12 \mathrm{~cm}$ dipole fed directly with a coaxial line, without a balun.

the top half and bottom half of the plot, the left and right halves of the pattern are clearly asymmetrical. Hence, the dipole is clearly unbalanced when fed directly with a coaxial line. Figure 158 illustrates the time-domain pattern for the dipole fed without a balun. As was the case with the $5 \mathrm{~cm}$ dipole, maximum radiation is along the broadside and minimum radiation occurs along the dipole axis. The measured time domain pulse shape, however, is different than that of the $5 \mathrm{~cm}$ dipole.

The measured patterns of a $12 \mathrm{~cm}$ dipole fed with the double-y balun are illustrated in Fig. 159. The measured pattern at $\lambda / 2$ agrees closely with the normalized amplitude pattern obtained via NEC for a balanced dipole. There is a slight deviation from NEC pattern between $180^{\circ}$ and $210^{\circ}$, which could be caused by scattering from the PVC support structure. Normalized amplitude patterns at $3 \lambda / 2$ and $5 \lambda / 2$ approximate the amplitude patterns generated via NEC for a balanced dipole at $3 \lambda / 2$ and $5 \lambda / 2$ respectively. While the double-y balun does not achieve perfect balancing at the above frequencies, comparing the measured azimuth patterns for the dipole fed with and without the balun clearly illustrates the improvement obtained via the use of the balun. The patterns agree much more closely to those of a perfectly balanced feed than those of an unbalanced feed. 


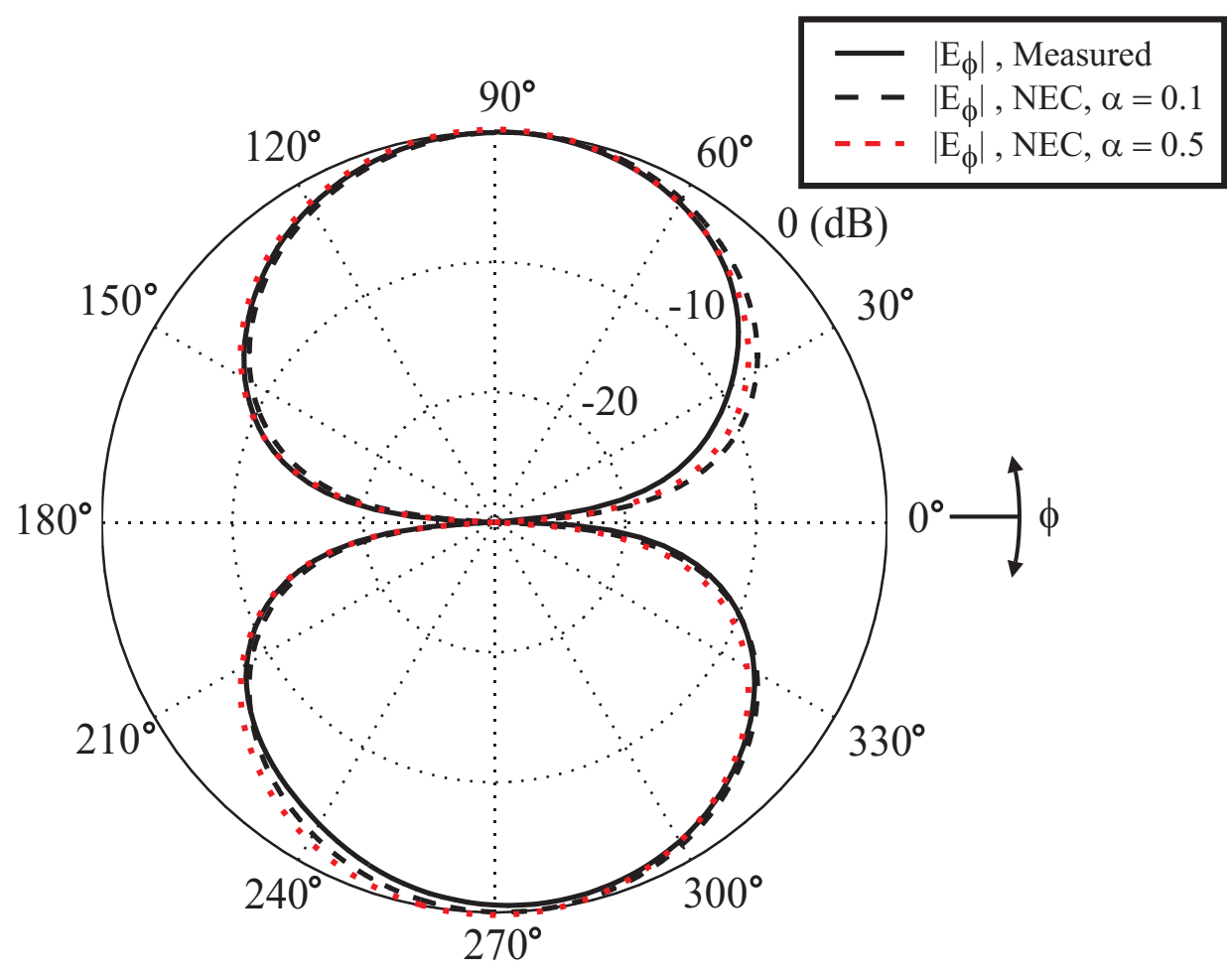

(a)

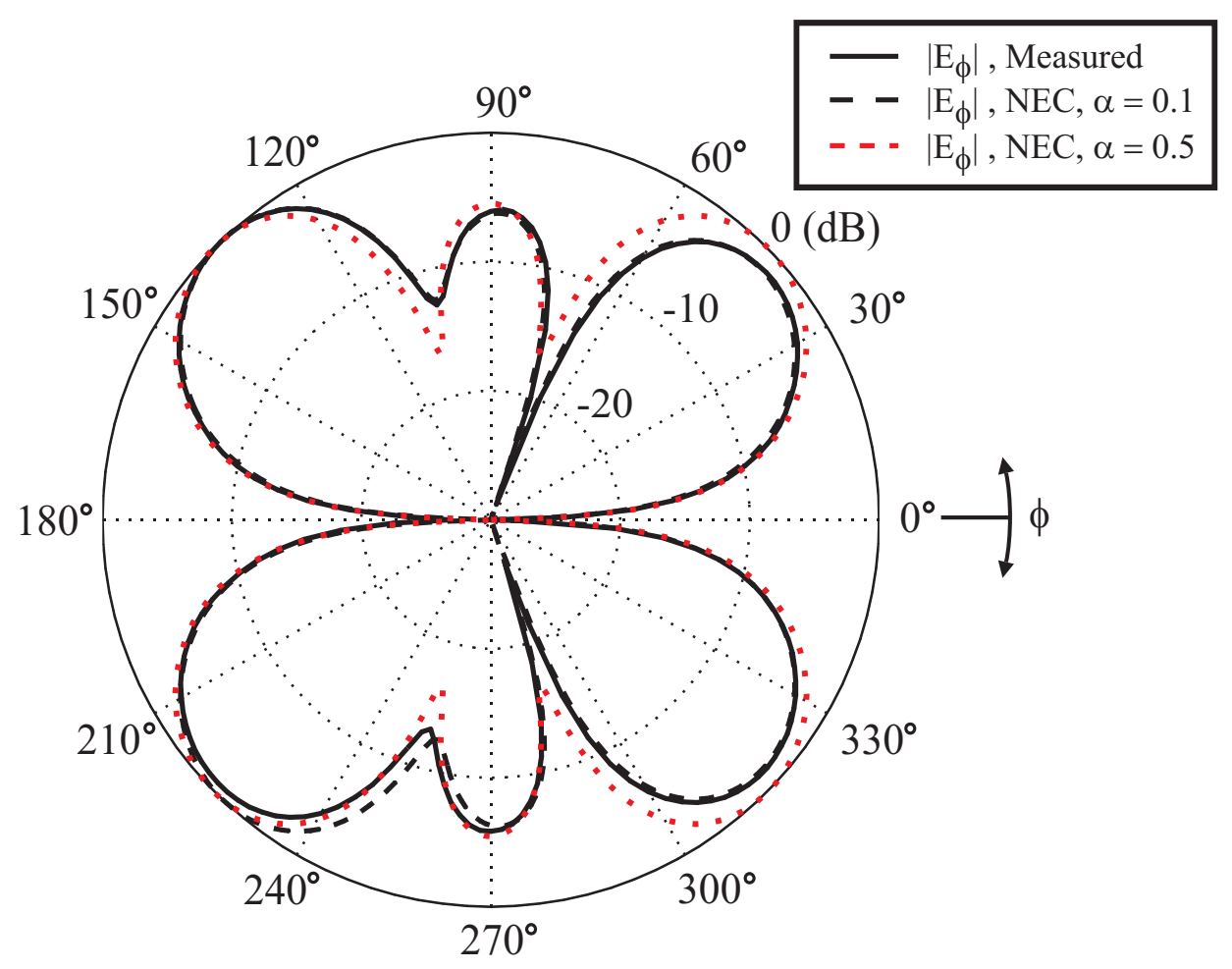

(b)

Figure 157: Measured vs. NEC (NEC model in Fig. 76 with $\alpha=0.1$ for unbalanced and $\alpha=0.5$ for balanced dipole) azimuth amplitude patterns for $12 \mathrm{~cm}$ dipole fed without a balun at (a) $\lambda / 2$, (b) $3 \lambda / 2$, and (c) $5 \lambda / 2$. 


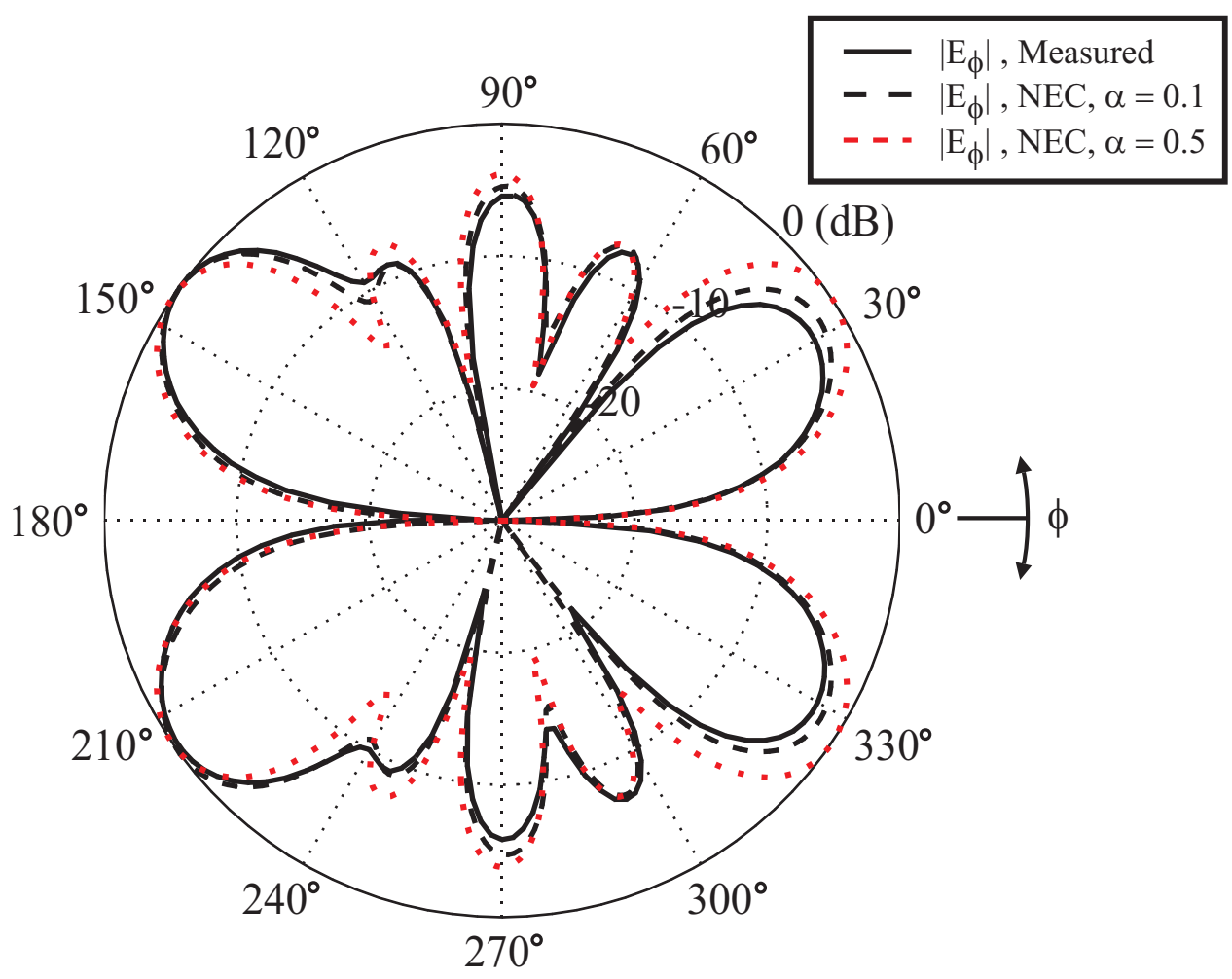

(c)

Figure 157: (Cont'd.) Measured vs. NEC (NEC model in Fig. 76 with $\alpha=0.1$ for unbalanced and $\alpha=0.5$ for balanced dipole) azimuth amplitude patterns for $12 \mathrm{~cm}$ dipole fed without a balun at (a) $\lambda / 2$, (b) $3 \lambda / 2$, and (c) $5 \lambda / 2$. 


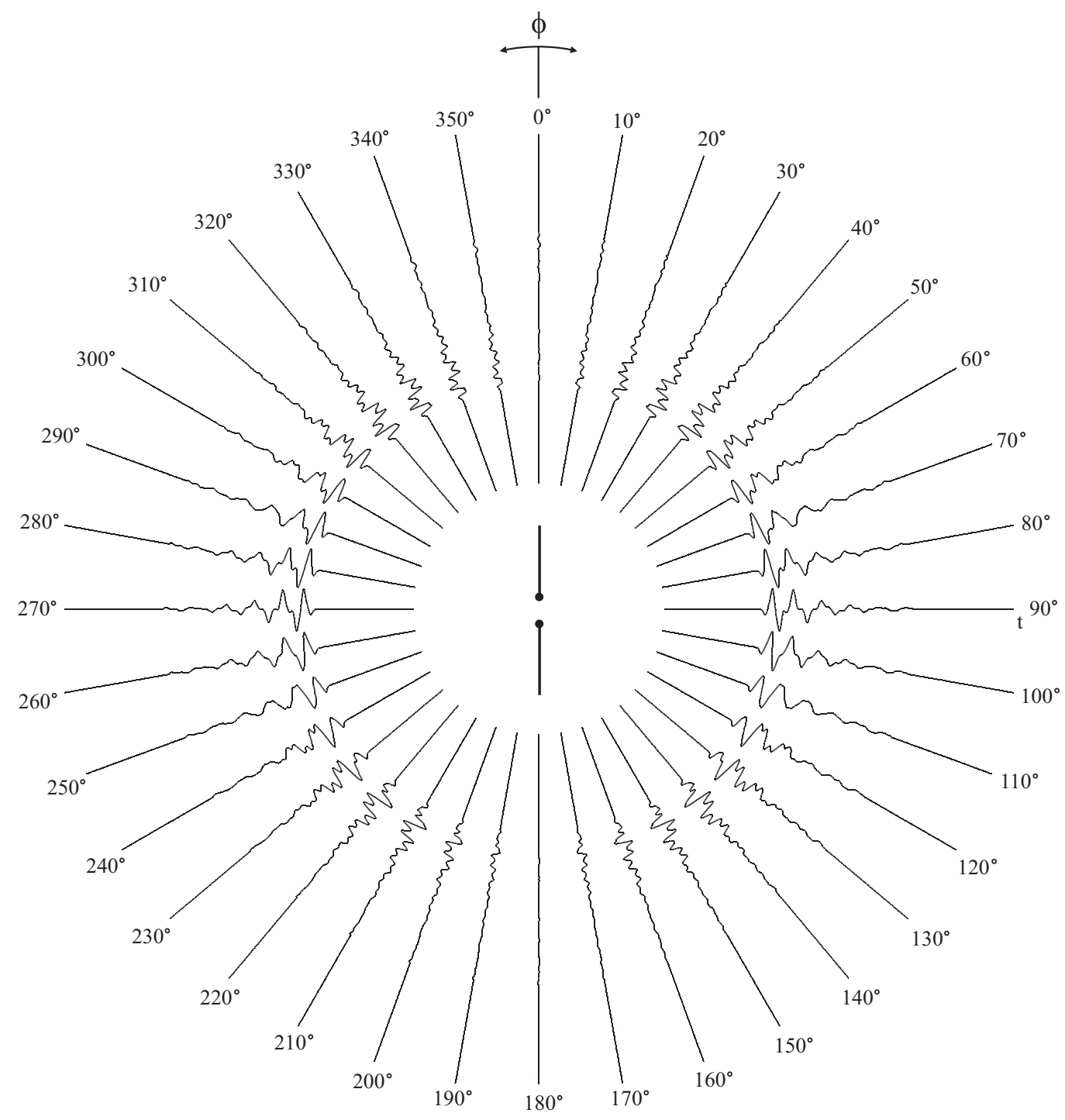

Figure 158: Time-domain plot of the measured voltage at the probe with an incident double differentiated Gaussian pulse (spectral peak at $3 \mathrm{GHz}$ ) transmitted by a $12 \mathrm{~cm}$ dipole fed without a balun. 


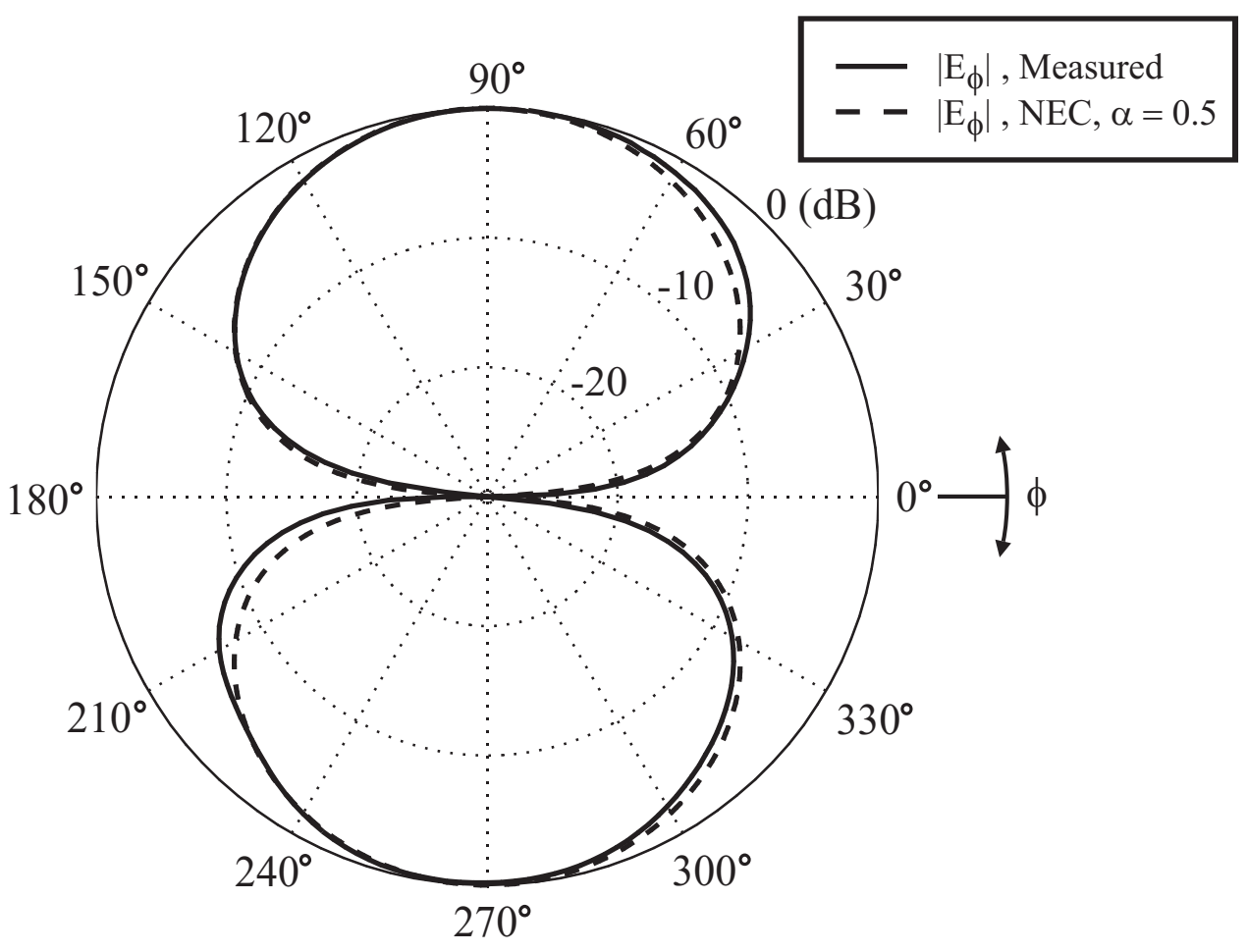

(a)

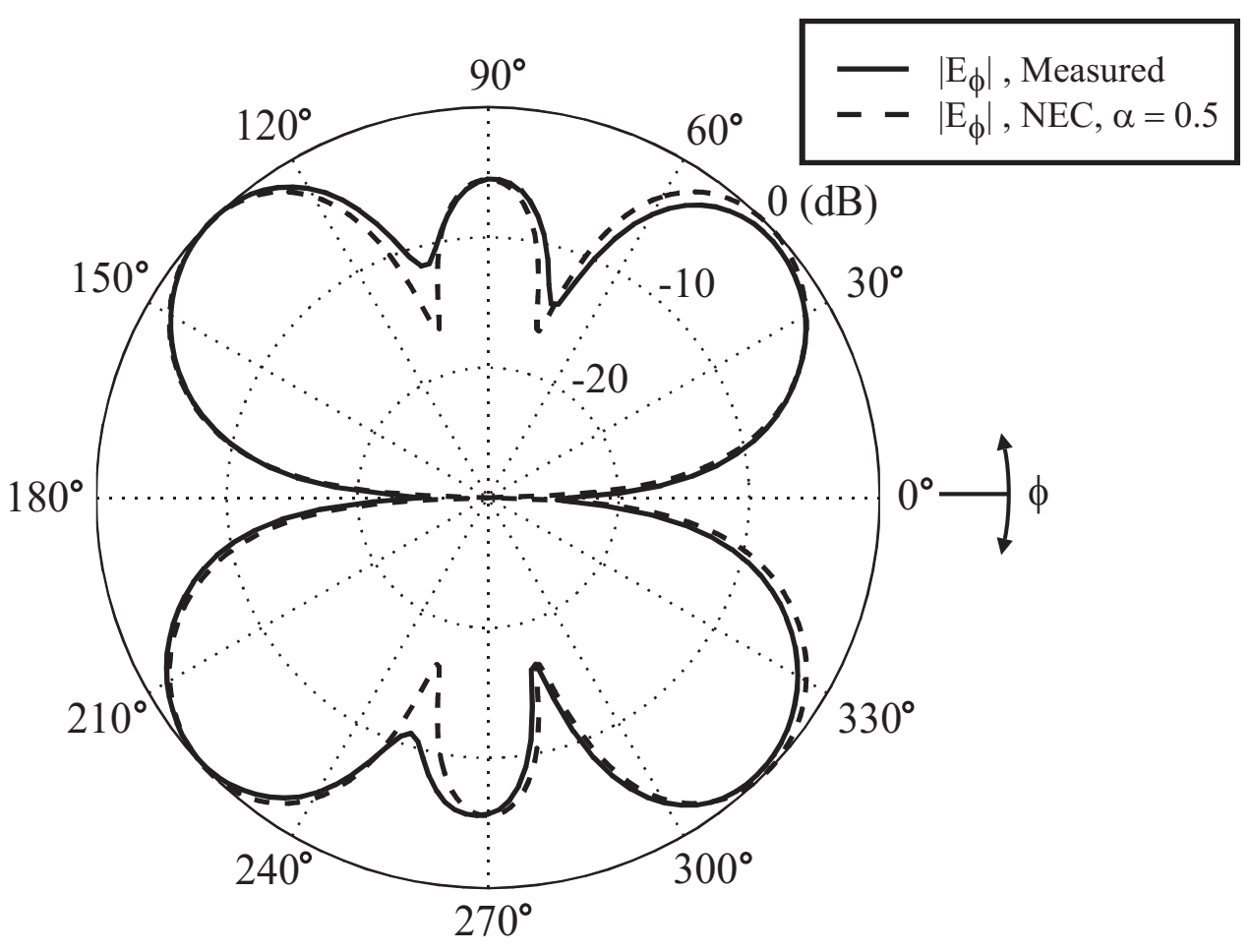

(b)

Figure 159: Measured vs. NEC (NEC model in Fig. 76 with $\alpha=0.5$ for balanced dipole) azimuth amplitude patterns for $12 \mathrm{~cm}$ dipole fed with the double-y balun at (a) $\lambda / 2$, (b) $3 \lambda / 2$, and (c) $5 \lambda / 2$. 


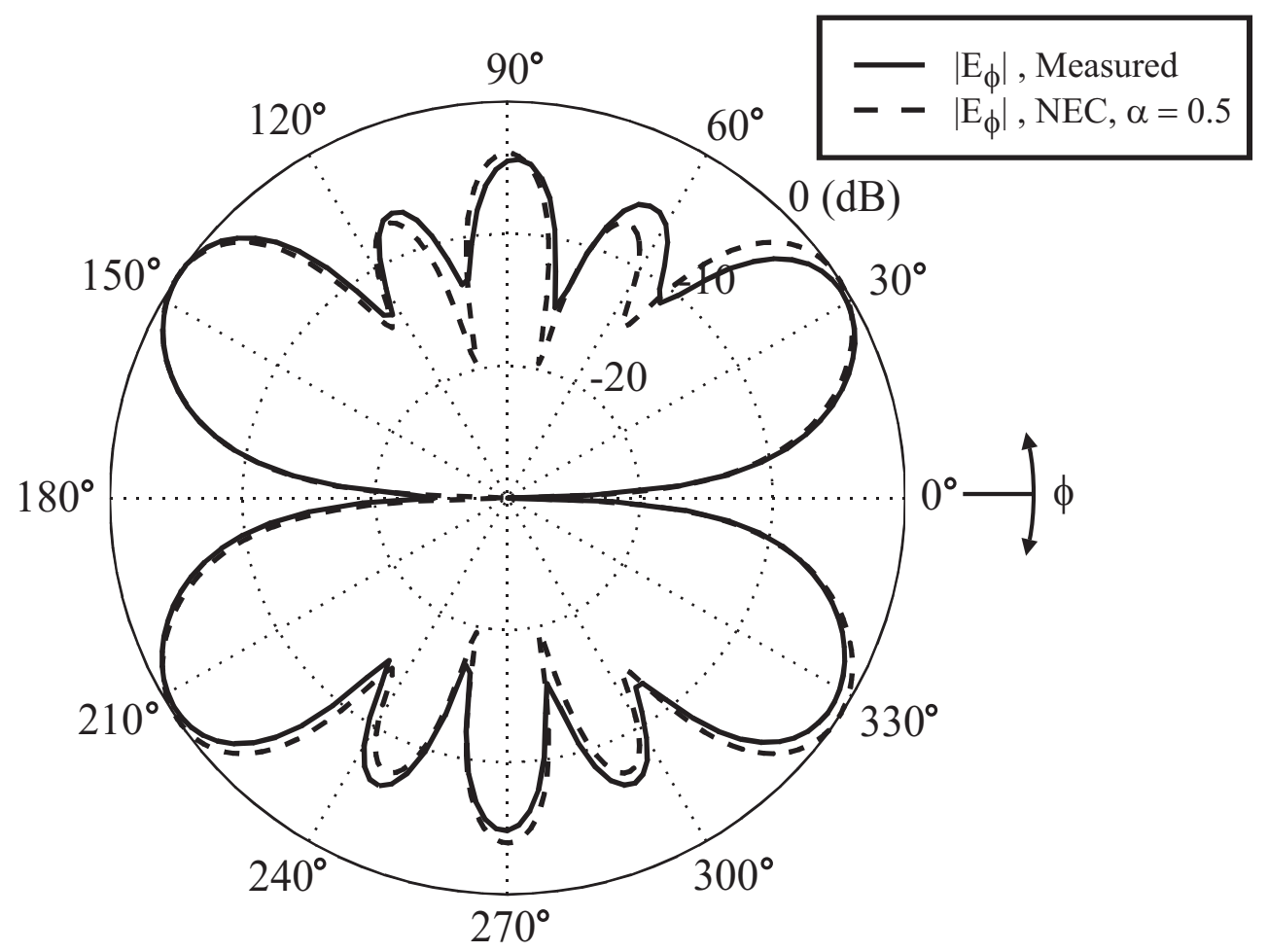

(c)

Figure 159: (Cont'd.) Measured vs. NEC azimuth amplitude patterns for $12 \mathrm{~cm}$ dipole fed with the double-y balun at (a) $\lambda / 2$, (b) $3 \lambda / 2$, and (c) $5 \lambda / 2$. 


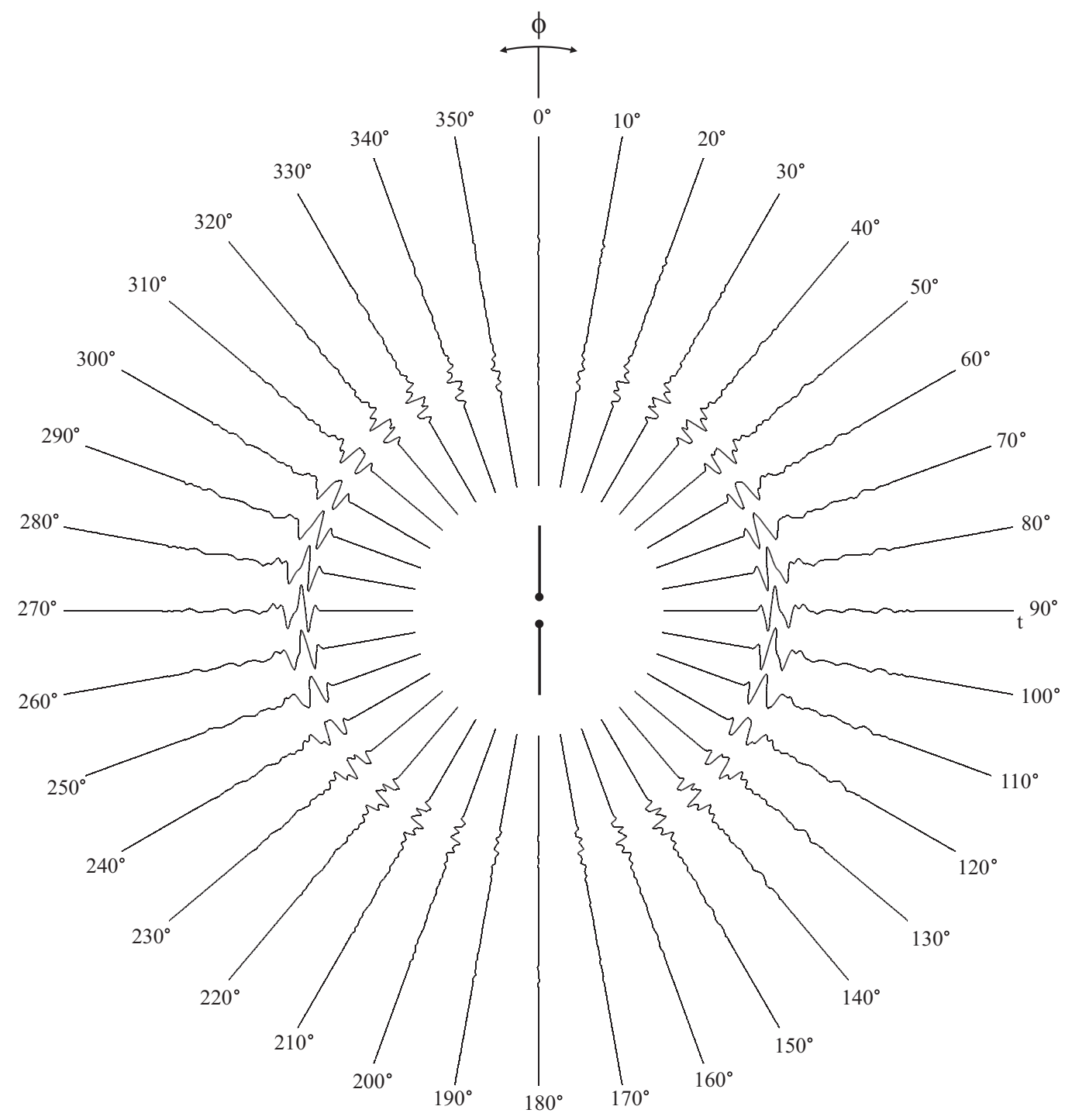

Figure 160: Time-domain plot of the measured voltage at the probe with an incident double differentiated Gaussian pulse (spectral peak at $3 \mathrm{GHz}$ ) transmitted by a $12 \mathrm{~cm}$ dipole fed with the double-y balun. 


\subsection{3 $5 \mathrm{~cm}$ Dipole Patterns with Optical Link}

Antenna patterns for a $5 \mathrm{~cm}$ dipole were measured along the elevation planes using the optical link described in Chapter 4. The use of an optical link confines radiation due to an unbalanced feed to the antenna and the laser receiver unit, thus allowing elevation pattern measurements to be conducted accurately. However, due to the bandwidth limitation imposed by the laser modulator, only $\lambda / 2$ pattern is analyzed for the $5 \mathrm{~cm}$ dipole. Measured elevation patterns are compared with results obtained from the NEC model in Fig. 161, which models the LR unit as well.

A block diagram of the new measurement setup is illustrated in Fig. 162; the setup in Fig. 142 was modified to include the optical link. Instead of feeding the AUT directly via the network analyzer, the network analyzer was used to provide RF modulation for the laser modulator unit (LM). The modulated light was transmitted via fiber optic cable to the laser receiver unit (LR), which demodulated the RF signal for transmission through the AUT. Figures 163a and 163b illustrate the AUT fed with the double-y balun and connected to the laser receiver (LR) unit. For measurements using the optical link, the LM unit was placed underneath the table onto which the positioner was mounted, as illustrated in Fig. 164. A hole was drilled through the table about the center of the rotary positioner, and the fiber optic cable from the LM unit was fed to the laser receiver (LR) unit through the hole. Both, the AUT (connected to the LR) and the probe were oriented to transmit and receive horizontal polarization.

Figure 165 illustrates the measured amplitude pattern along the elevation plane for a $5 \mathrm{~cm}$ dipole fed without a balun, using the optical link in the measurement system. The measured pattern agrees close enough to the NEC model in Fig. 161 (with $\alpha=0.1$ to model an unbalanced dipole) to illustrate that the arms of the dipole are unbalanced. The measured time-domain pattern is illustrated in Fig. 166. This pattern clearly illustrates the lack of symmetry, particularly between the waveforms 


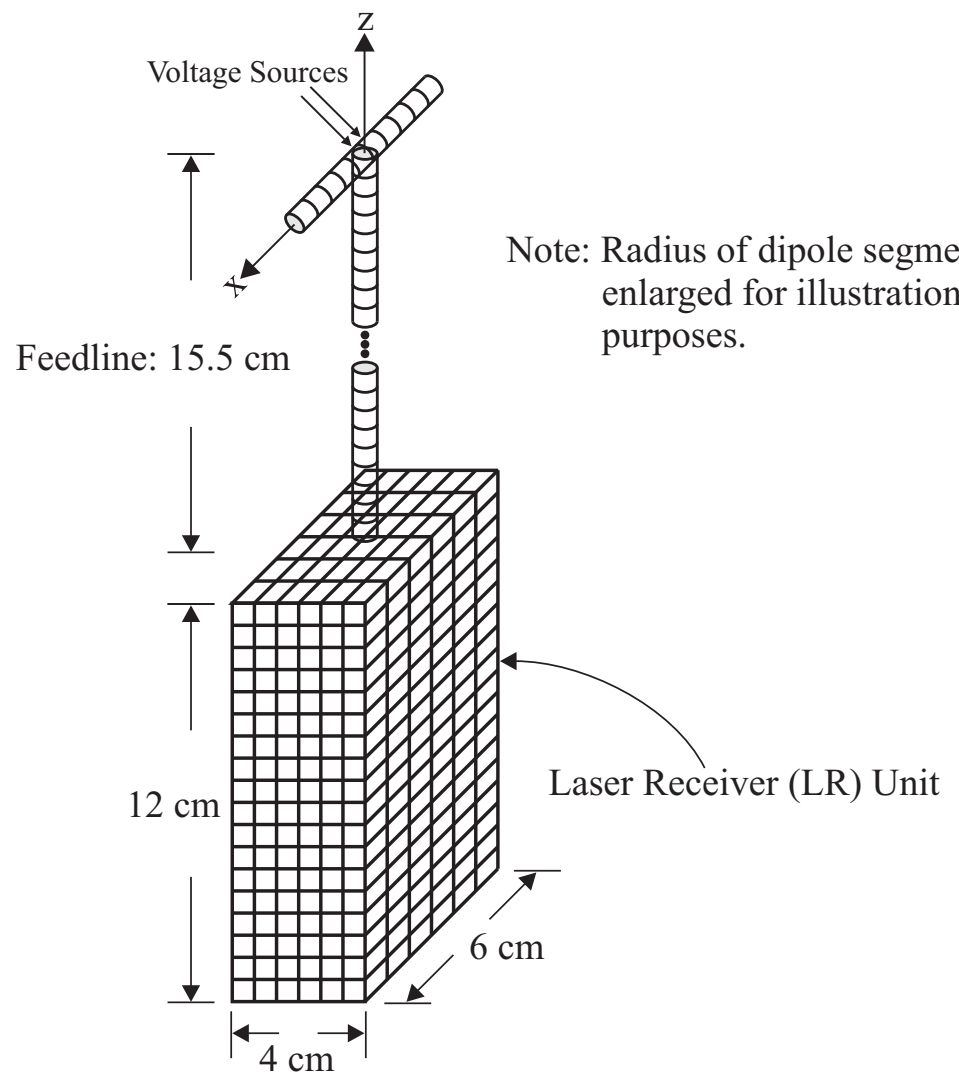

Figure 161: NEC model in Fig. 76 expanded to include laser receiver (LR) unit.

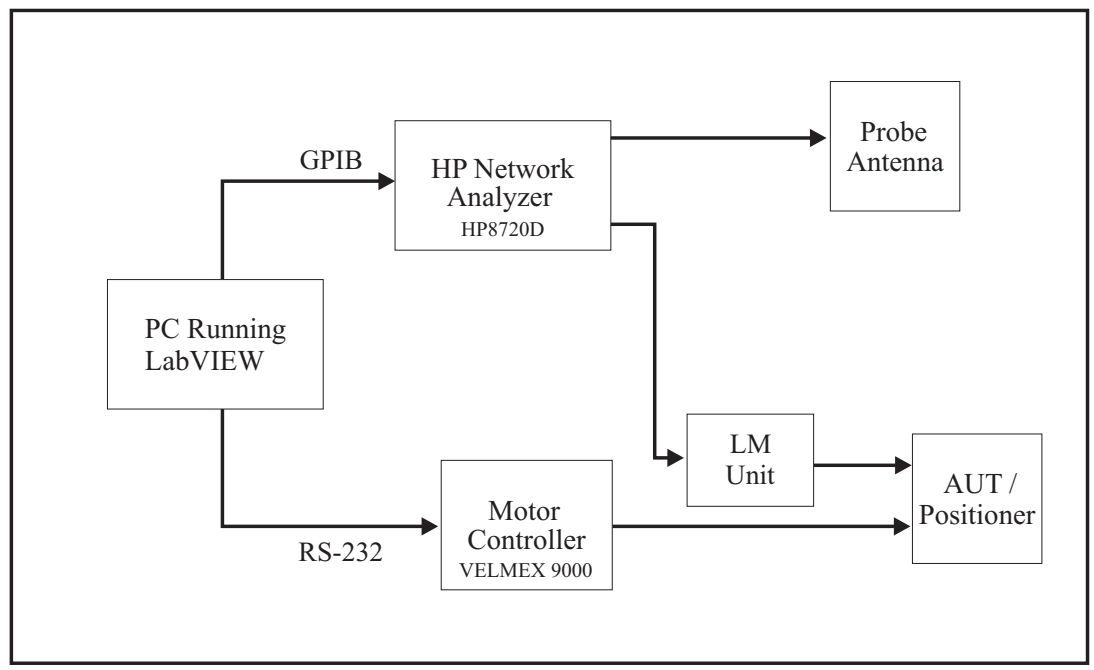

Figure 162: Block diagram of modified measurement system for conducting pattern measurements along elevation plane. 


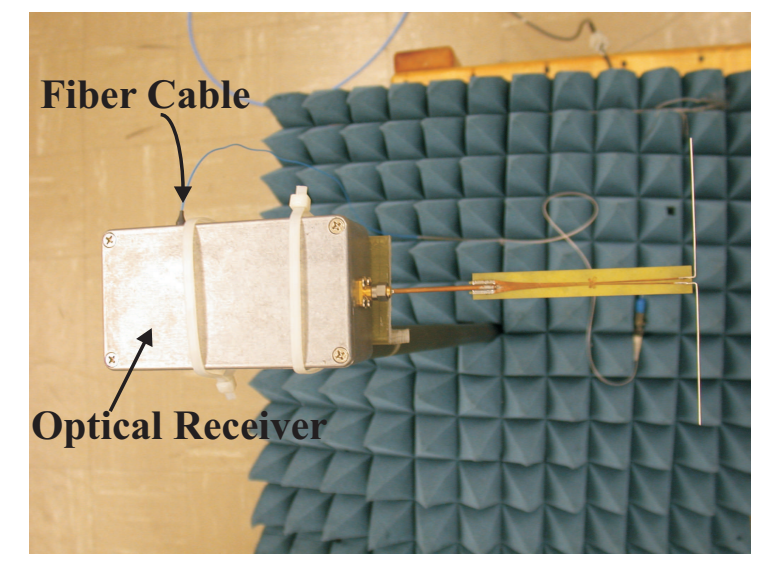

(a)

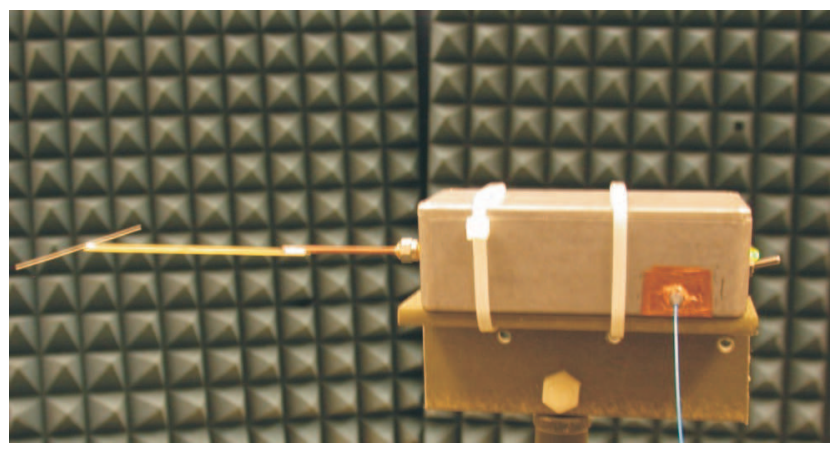

(b)

Figure 163: Illustration of (a) top view and (b) side view of AUT connected to the laser receiver unit mounted on the rotary positioner.

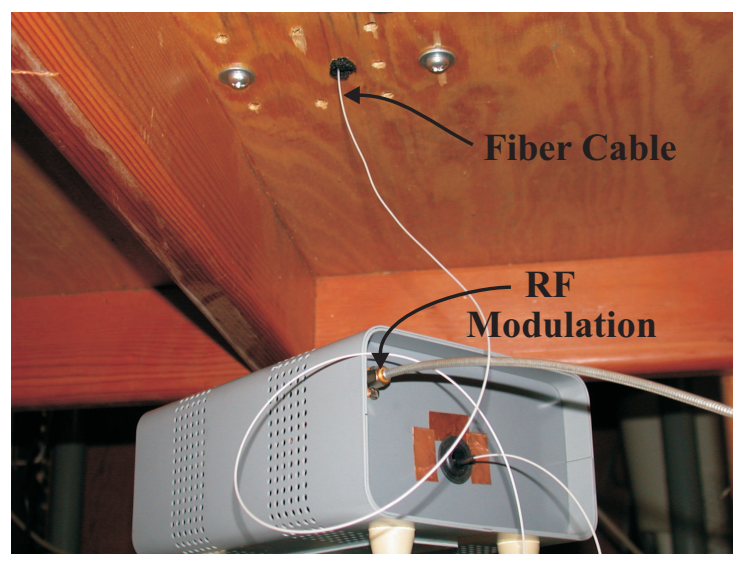

Figure 164: Illustration of laser modulator (LM) unit, placed underneath the positioner during antenna pattern measurement using the optical link detailed in Chapter 4 . 
at $160^{\circ}$ and $200^{\circ}$. To investigate the lack of symmetry more closely, waveforms at $20^{\circ}$ and $340^{\circ}$ are illustrated in Fig. 167. These angles were chosen because at $20^{\circ}$ and $340^{\circ}$ both antennas are facing each other; the LR unit does not shadow the AUT. It is seen that there is a difference in the amplitudes of the initial waveforms near 7 ns. Also, there is a noticeable discrepancy between the waveforms at 8 ns. This is likely caused by ringing of the unbalanced dipole arms. Reflections from the feedline are observed past 9 ns.

Figure 168 illustrates the measured amplitude pattern along the elevation plane for a $5 \mathrm{~cm}$ dipole fed with the double-y balun. The measured pattern is compared with the pattern generated via the NEC model in Fig. 161, with $\alpha=0.5$ to model a balanced dipole feed. While there is some discrepancy between the measured and theoretical patterns, there is sufficient agreement between both patterns to illustrate the improvement obtained with the double-y balun. The measured pattern in Fig. 168 clearly agrees better with the NEC pattern for the balanced case than that generated for the unbalanced case in Fig. 165. The discrepancy in the patterns is likely caused by the difference in electrical lengths between the feedline in NEC $(15.5 \mathrm{~cm})$ and the actual feedline with the double-y balun (common-mode wave travels slower along the double-y balun because of the FR4 substrate). The measured time-domain pattern along the elevation plane is illustrated in Fig. 169. This pattern illustrates the radiated field along the elevation plane in the time domain. Symmetry in the timedomain pattern is investigated in Fig. 170. As seen from Fig. 170, the amplitudes of the initial pulse (before ringing) agree closely. Since the balun does not achieve perfect balance at all frequencies, smaller reflections are observed; these reflections could result from the balun junction.

The amplitude pattern of a $5 \mathrm{~cm}$ dipole fed the sleeve balun was also measured, as illustrated in Fig. 171. There is good agreement between the measured pattern and that obtained via the NEC model in Fig. 161. From Fig. 171, it is evident 
that the sleeve balun improves the feed balance; the measured pattern agrees more closely with the pattern obtained via the NEC model in Fig. 161 with a balanced dipole, than with the pattern of an unbalanced dipole. The time-domain pattern is illustrated in Fig. 172. There is improvement in the overall symmetry of the pattern when compared to Fig. 166. The symmetry in the time domain is investigated more closely in Fig. 173 , at $20^{\circ}$ and $340^{\circ}$. While Fig. 171 illustrates a balanced dipole pattern when fed with the sleeve balun, it is difficult to discern between the timedomain patterns for an unbalanced dipole and a dipole fed with the sleeve balun. This is because the sleeve balun is a narrowband balun, and ideally achieves balance at odd multiples of a single frequency. The time-domain pattern in Fig. 173 contains the response of the dipole fed with the sleeve balun over a wide range of frequencies. Reflections are observed between 9 ns and 10.5 ns; these ripples are likely caused by the shorted quarter-wavelength sleeve balun as well as the feedline. 


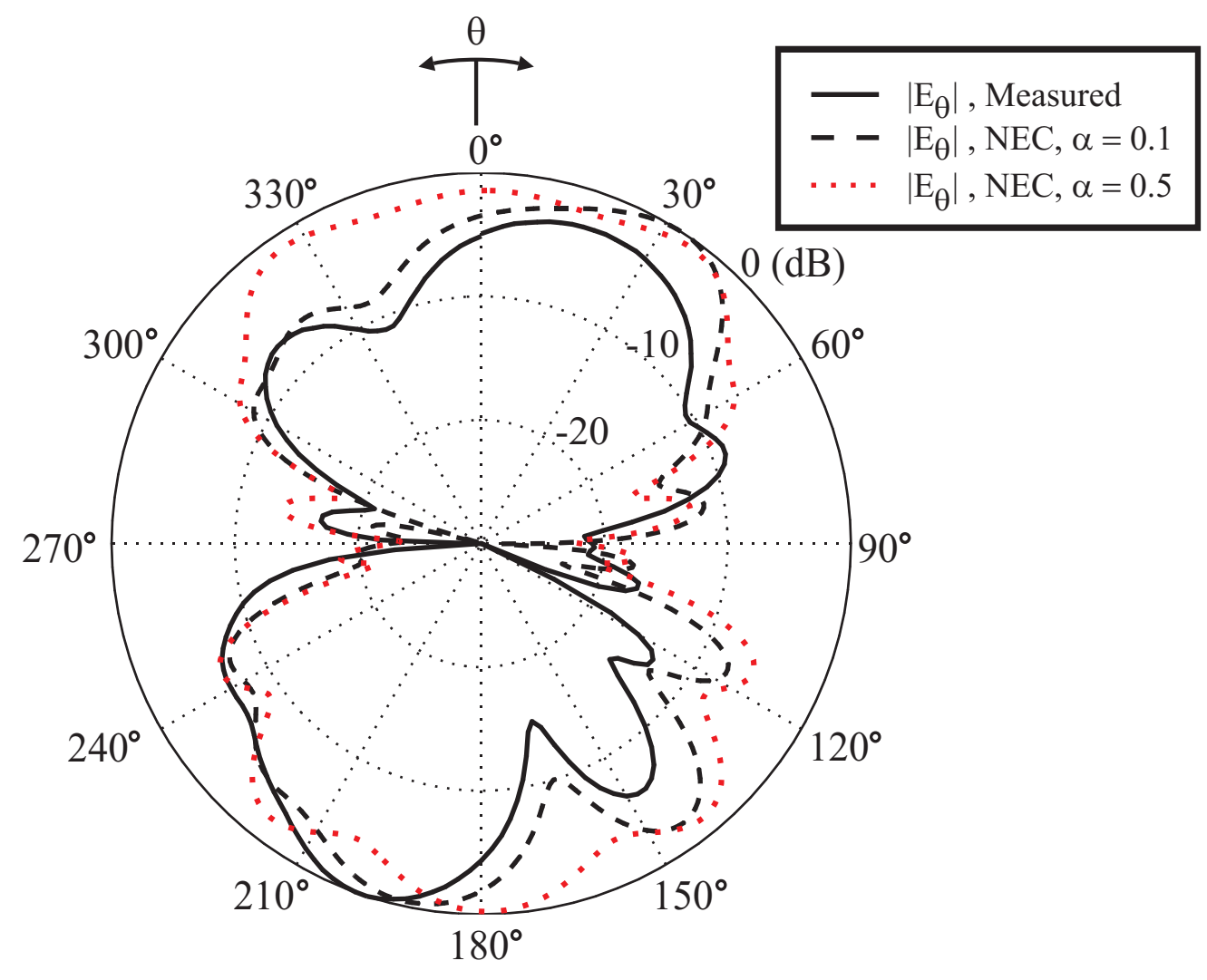

Figure 165: Measured vs. NEC (NEC model in Fig. 161 with $\alpha=0.1$ for unbalanced and $\alpha=0.5$ for balanced dipole) elevation amplitude pattern for $5 \mathrm{~cm}$ dipole fed without a balun at $\lambda / 2$. 


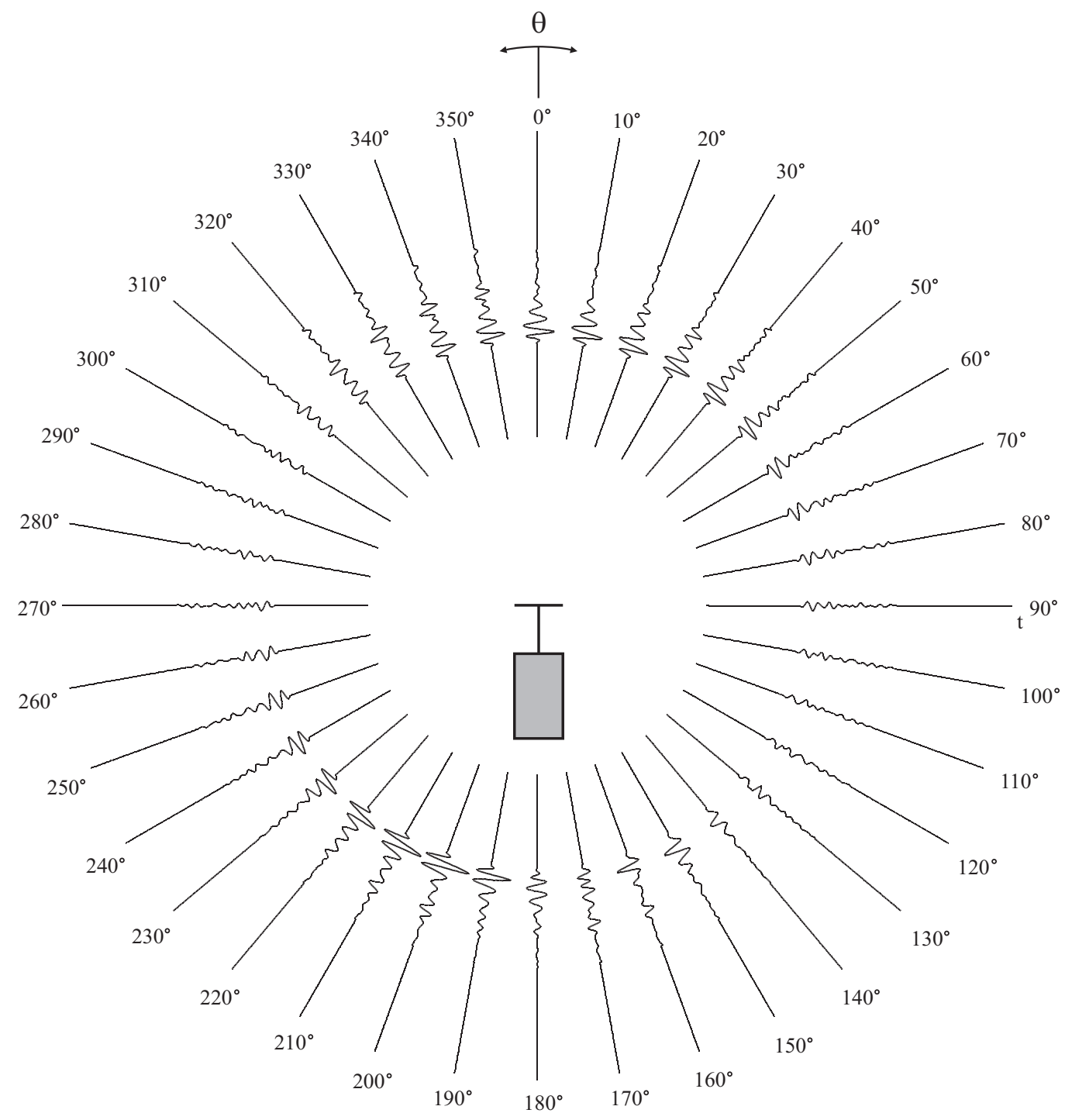

Figure 166: Time-domain pattern of the measured voltage at the probe with an incident double differentiated Gaussian pulse (spectral peak at $3 \mathrm{GHz}$ ) transmitted by a $5 \mathrm{~cm}$ dipole fed without a balun. 


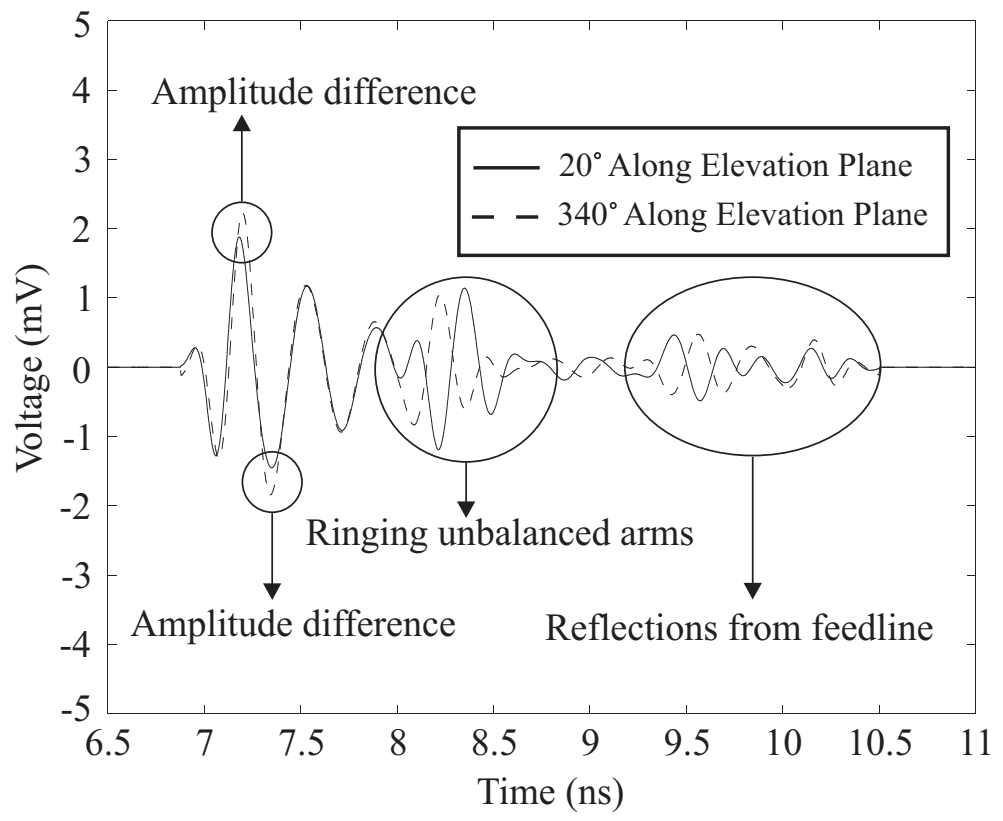

Figure 167: Plot comparing time-domain waveforms from Fig. 166 at $20^{\circ}$ and $340^{\circ}$.

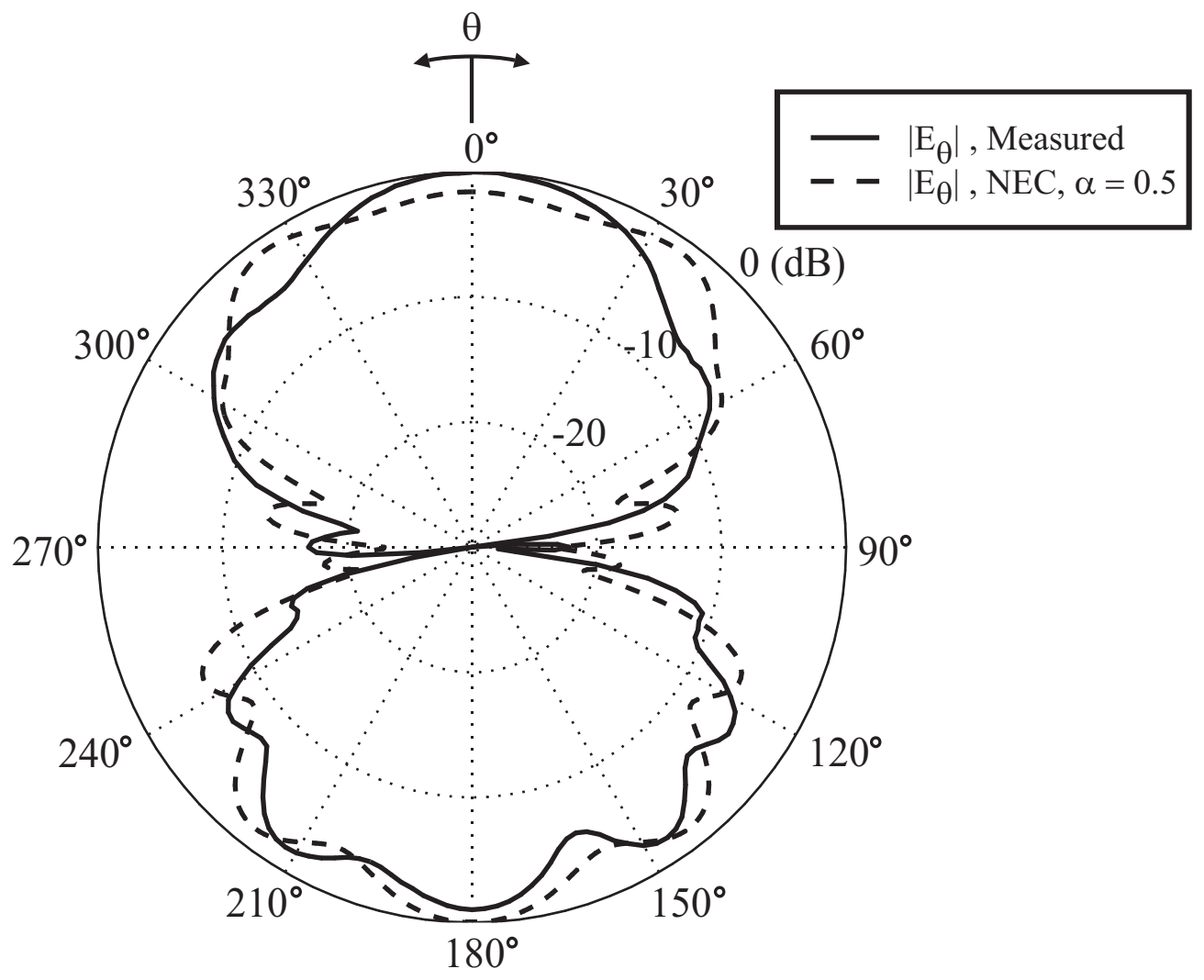

Figure 168: Measured vs. NEC (NEC model in Fig. 161 with $\alpha=0.5$ for balanced dipole) elevation amplitude pattern for $5 \mathrm{~cm}$ dipole fed with the double-y balun at $\lambda / 2$. 


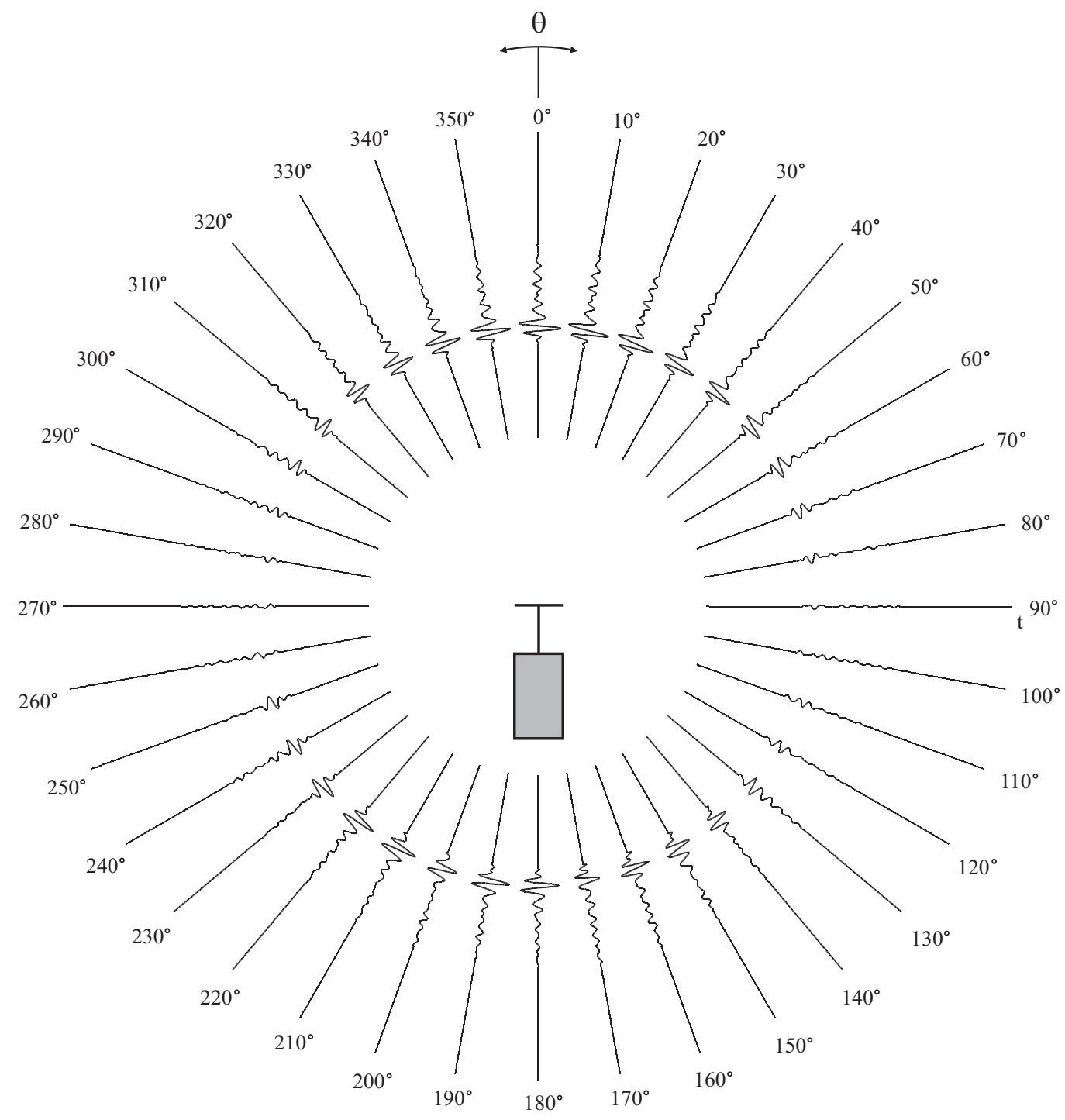

Figure 169: Time-domain pattern of the measured voltage at the probe with an incident double differentiated Gaussian pulse (spectral peak at $3 \mathrm{GHz}$ ) transmitted by a $5 \mathrm{~cm}$ dipole fed with the double-y balun. 


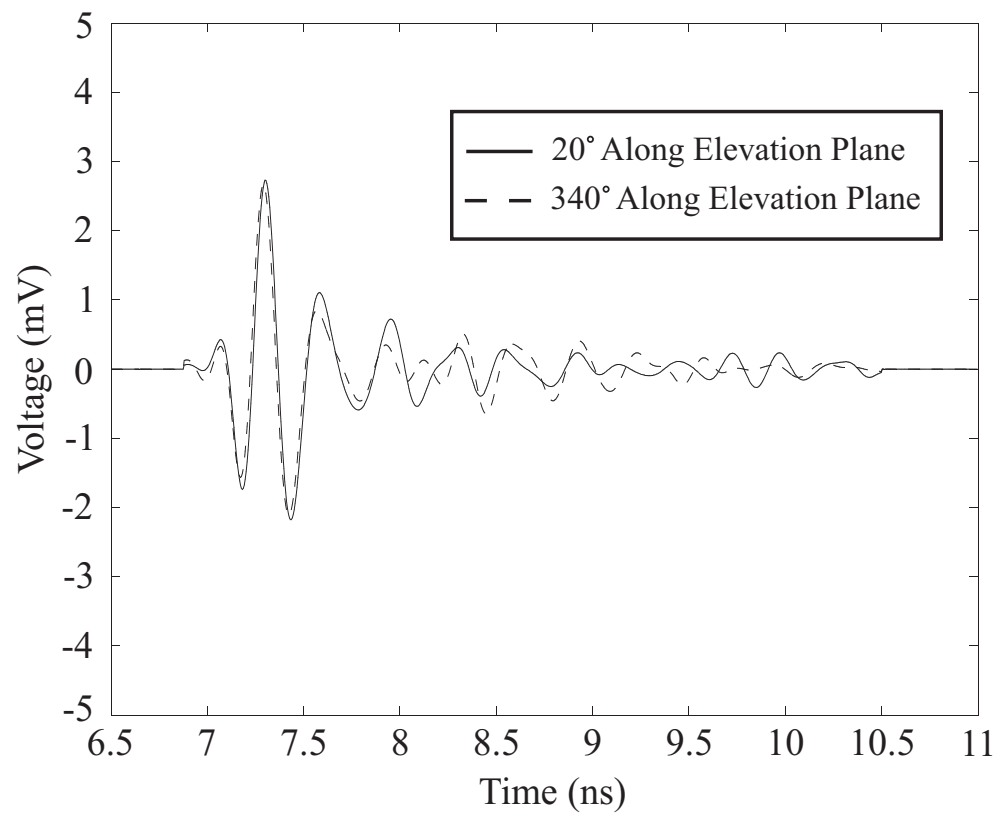

Figure 170: Plot comparing time-domain waveforms from Fig. 169 at $20^{\circ}$ and $340^{\circ}$.

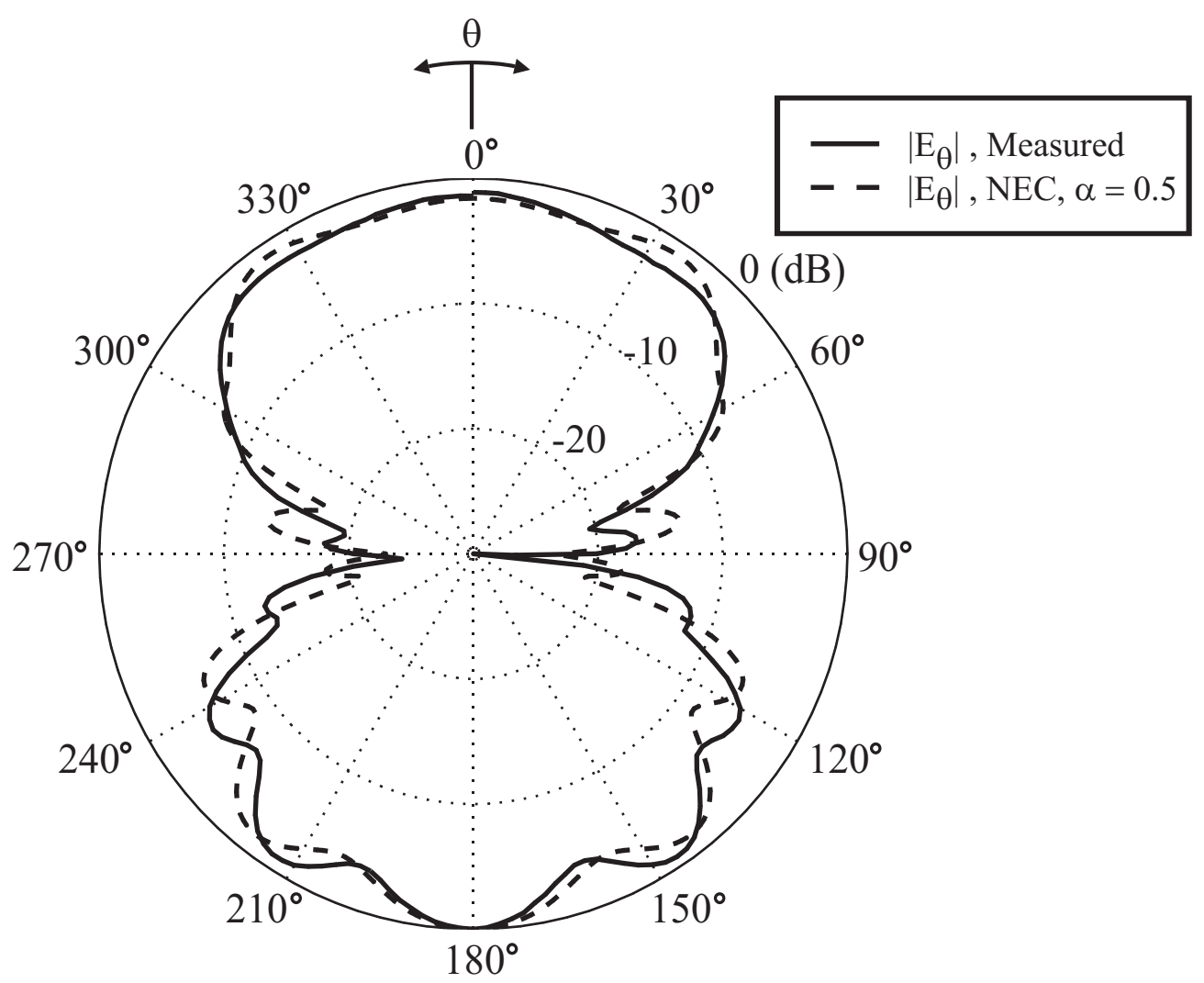

Figure 171: Measured vs. NEC (NEC model in Fig. 161 with $\alpha=0.5$ for balanced dipole) elevation amplitude pattern for $5 \mathrm{~cm}$ dipole fed with the sleeve balun at $\lambda / 2$. 


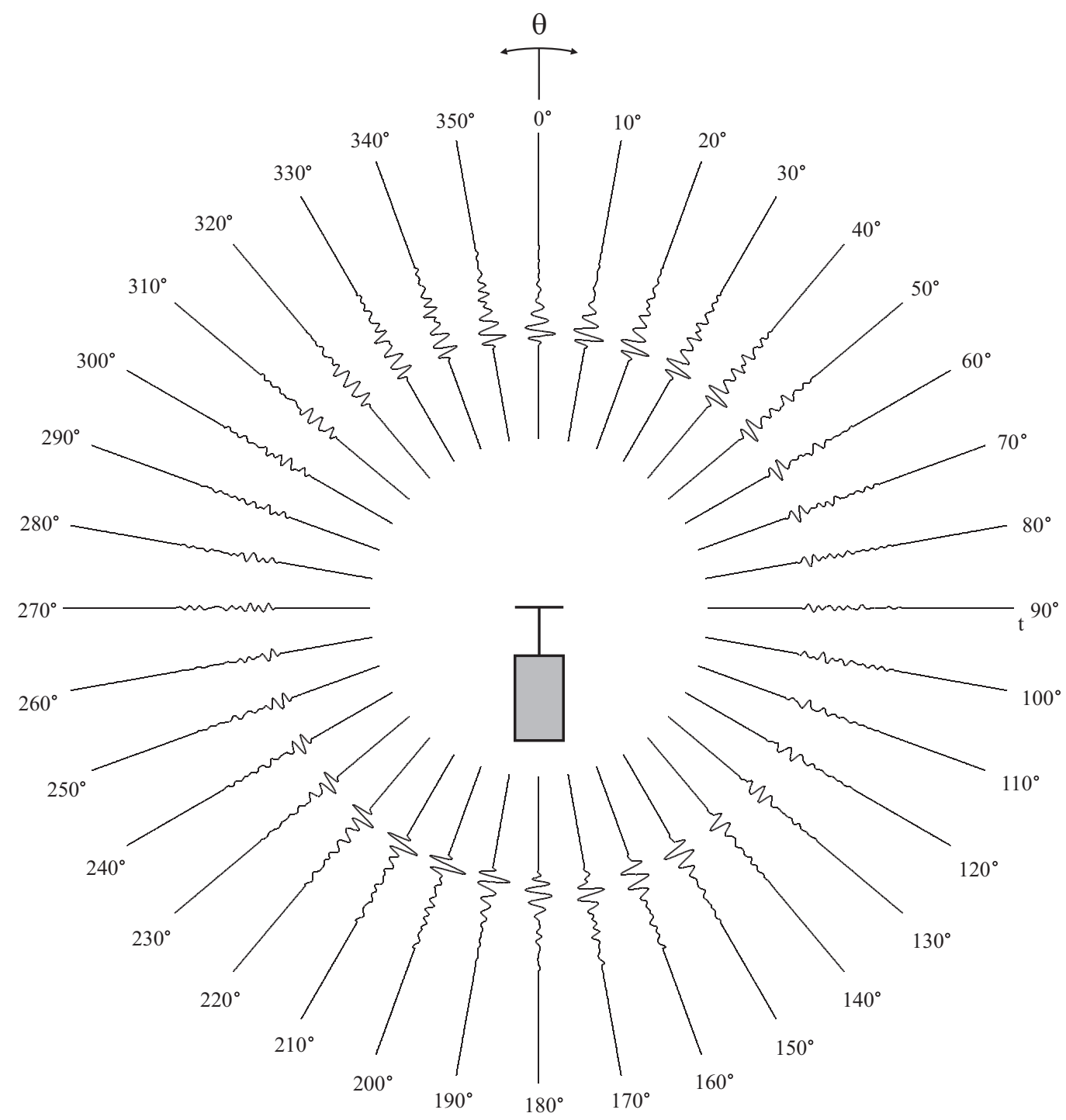

Figure 172: Time-domain pattern of the measured voltage at the probe with an incident double differentiated Gaussian pulse (spectral peak at $3 \mathrm{GHz}$ ) transmitted by a $5 \mathrm{~cm}$ dipole fed with the sleeve balun. 


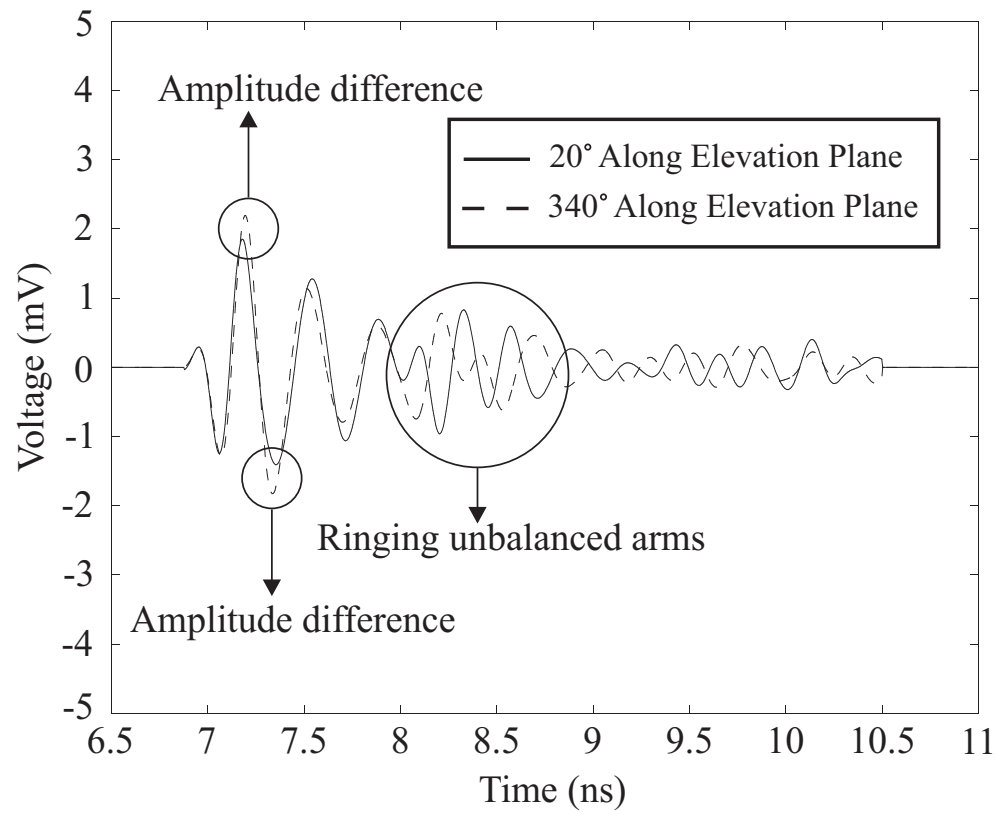

Figure 173: Plot comparing time-domain waveforms from Fig. 172 at $20^{\circ}$ and $340^{\circ}$. 


\subsection{4 $12 \mathrm{~cm}$ Dipole Patterns with Optical Link}

Amplitude patterns were measured along the elevation plane for the $12 \mathrm{~cm}$ dipole fed with and without the double-y balun. The measurement setup was the same as that used in Section 5.2.3. A 3.5 ns time window was allowed before time gating the measured data; reflections from the feedline were computed to occur within the $3.5 \mathrm{~ns}$ time window. This time window could be increased if measurements are conducted in an anechoic chamber.

Figure 174 illustrates measured patterns for the $12 \mathrm{~cm}$ dipole fed directly without a balun. The patterns are compared with the NEC model in Fig. 161 with a $12 \mathrm{~cm}$ unbalanced dipole $(\alpha=0.1)$. While there is some discrepancy between the measured and numerical patterns, the measured patterns clearly illustrate that the arms of the dipole are unbalanced; there is lack of symmetry in the patterns. The measured time-domain pattern is illustrated in Fig. 175. Lack of symmetry is observed in the resulting patterns; the waveform at $30^{\circ}$ differs substantially from that at $330^{\circ}$. This lack of symmetry is illustrated in Fig. 176, where the patterns at $30^{\circ}$ and $330^{\circ}$ are clearly different.

Figure 177 illustrates measured patterns for the $12 \mathrm{~cm}$ dipole fed with the doubley balun. The patterns are compared with the NEC model in Fig. 161 with a $12 \mathrm{~cm}$ balanced dipole $(\alpha=0.5)$. As was the case with the unbalanced dipole, there is some discrepancy between the measured and numerical patterns. However, the patterns agree sufficiently to illustrate the improvement in the pattern when compared with results obtained for the unbalanced dipole in Fig. 174. The measured time-domain pattern for the $12 \mathrm{~cm}$ dipole fed with the double-y balun is illustrated in Fig. 178. The pattern illustrates better symmetry than the pattern for the $12 \mathrm{~cm}$ dipole without a balun, in Fig. 175. Symmetry in the pattern is better illustrated in Fig. 179, where it is seen that the patterns agree closely. Hence, improvement in the symmetry of the time-domain pattern is seen with the use of the double-y balun. 

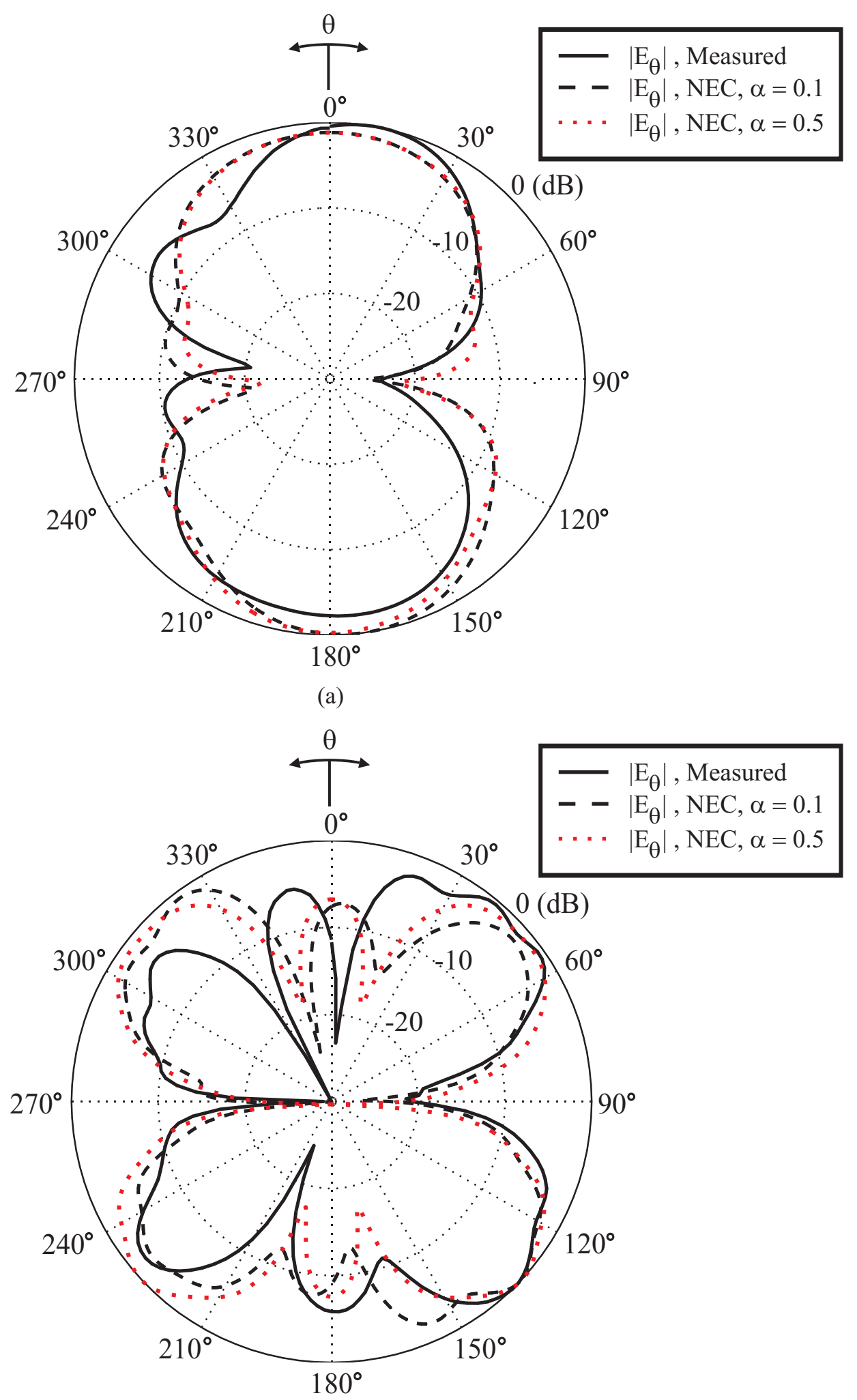

(b)

Figure 174: Measured vs. NEC (NEC model in Fig. 161 with $\alpha=0.5$ for balanced dipole) elevation amplitude pattern for $12 \mathrm{~cm}$ dipole fed without a balun at (a) $\lambda / 2$ and (b) $3 \lambda / 2$. 


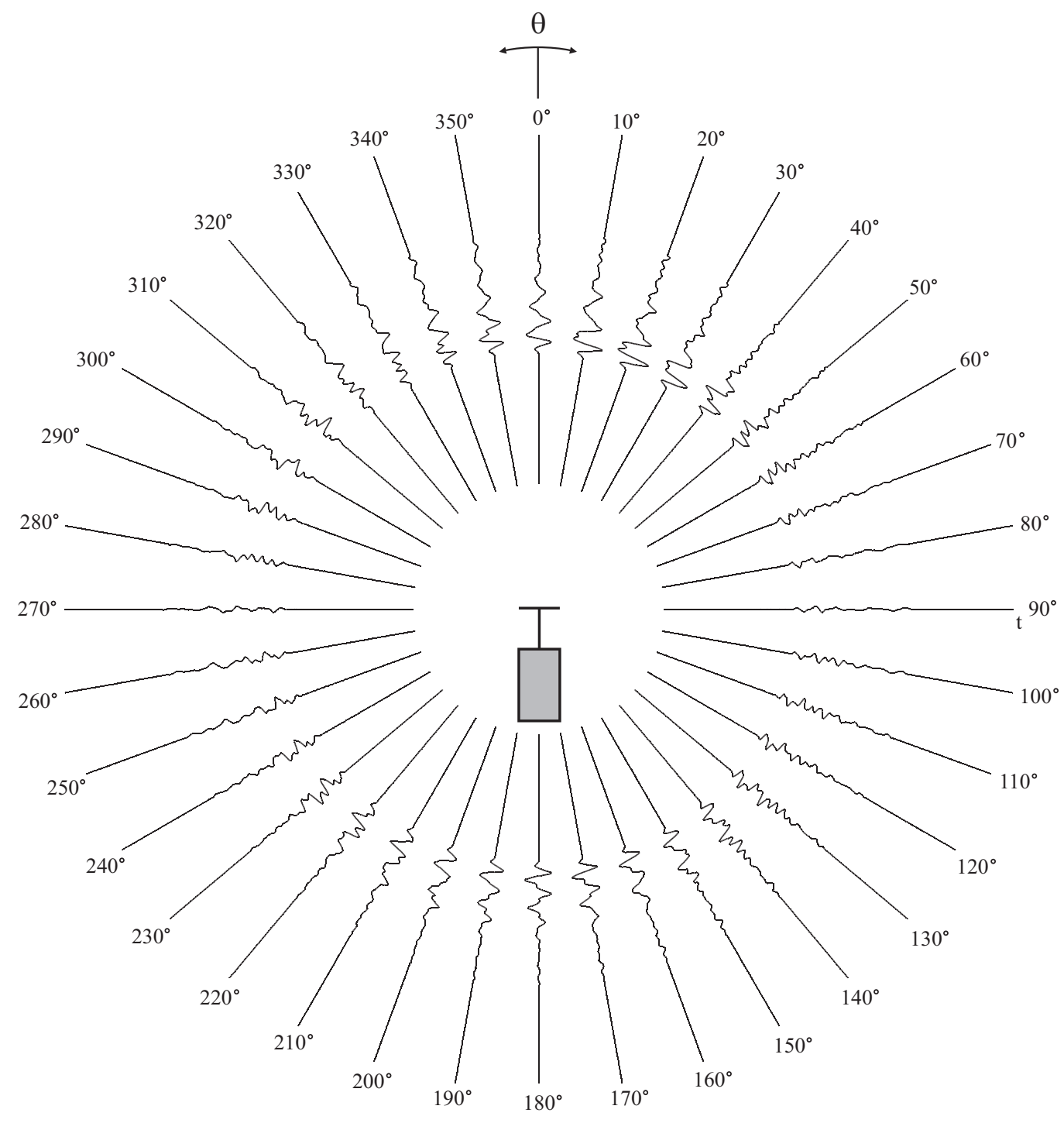

Figure 175: Time-domain pattern of the measured voltage at the probe along the elevation plane, with an incident double differentiated Gaussian pulse (spectral peak at $3 \mathrm{GHz}$ ) transmitted by a $5 \mathrm{~cm}$ dipole fed without a balun. 


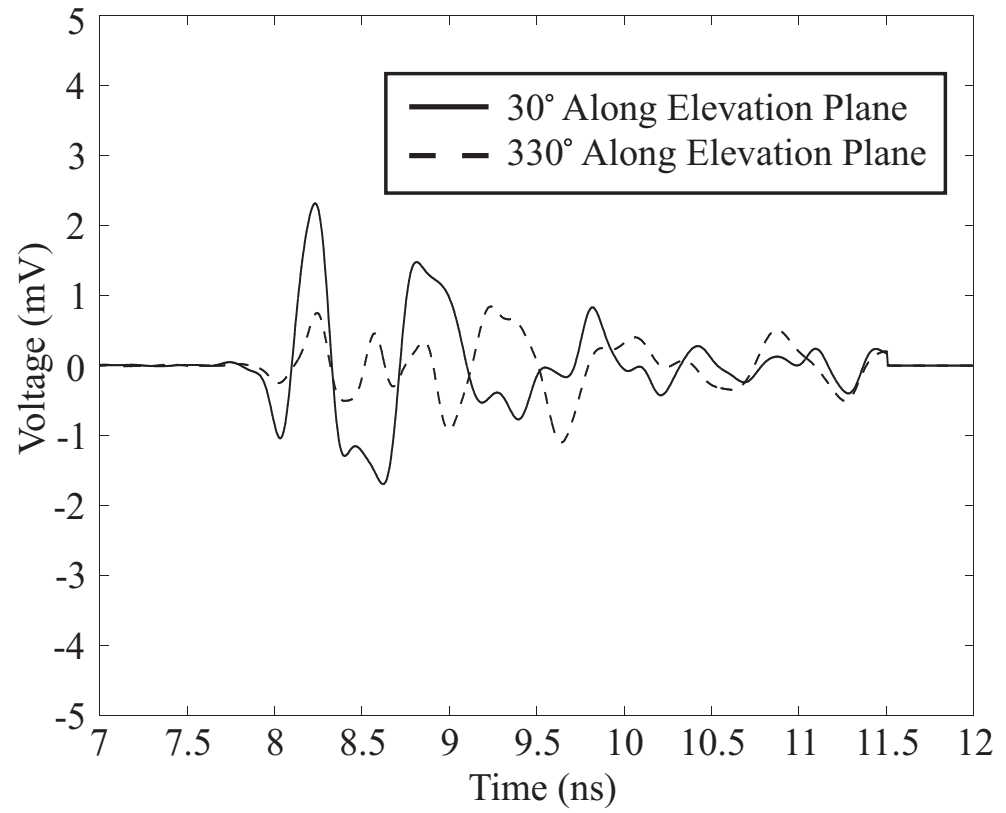

Figure 176: Plot comparing time-domain waveforms from Fig. 175 at $30^{\circ}$ and $330^{\circ}$. 

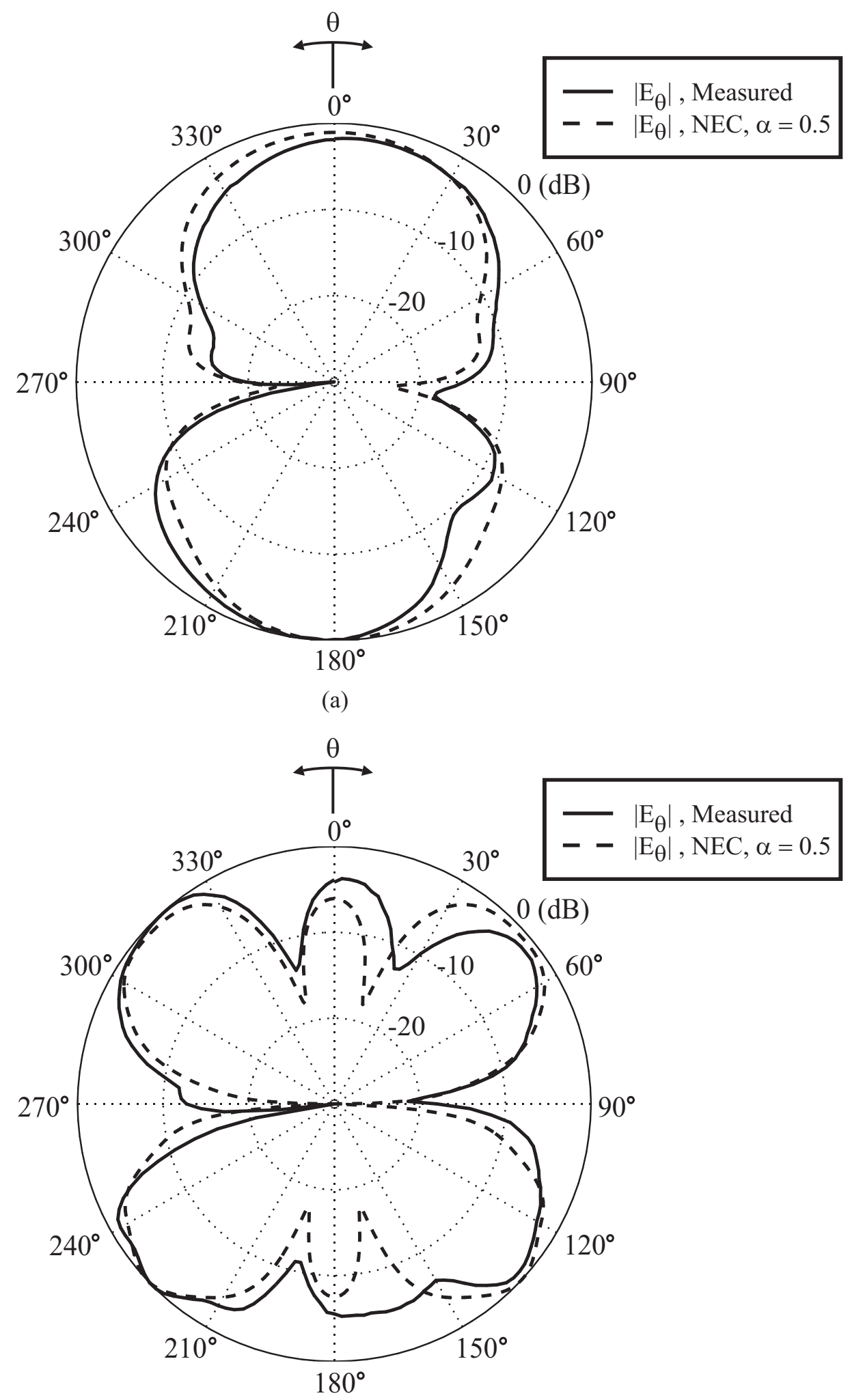

(b)

Figure 177: Measured vs. NEC (NEC model in Fig. 161 with $\alpha=0.5$ for balanced dipole) elevation amplitude pattern for $12 \mathrm{~cm}$ dipole fed with the double-y balun at (a) $\lambda / 2$ and (b) $3 \lambda / 2$. 


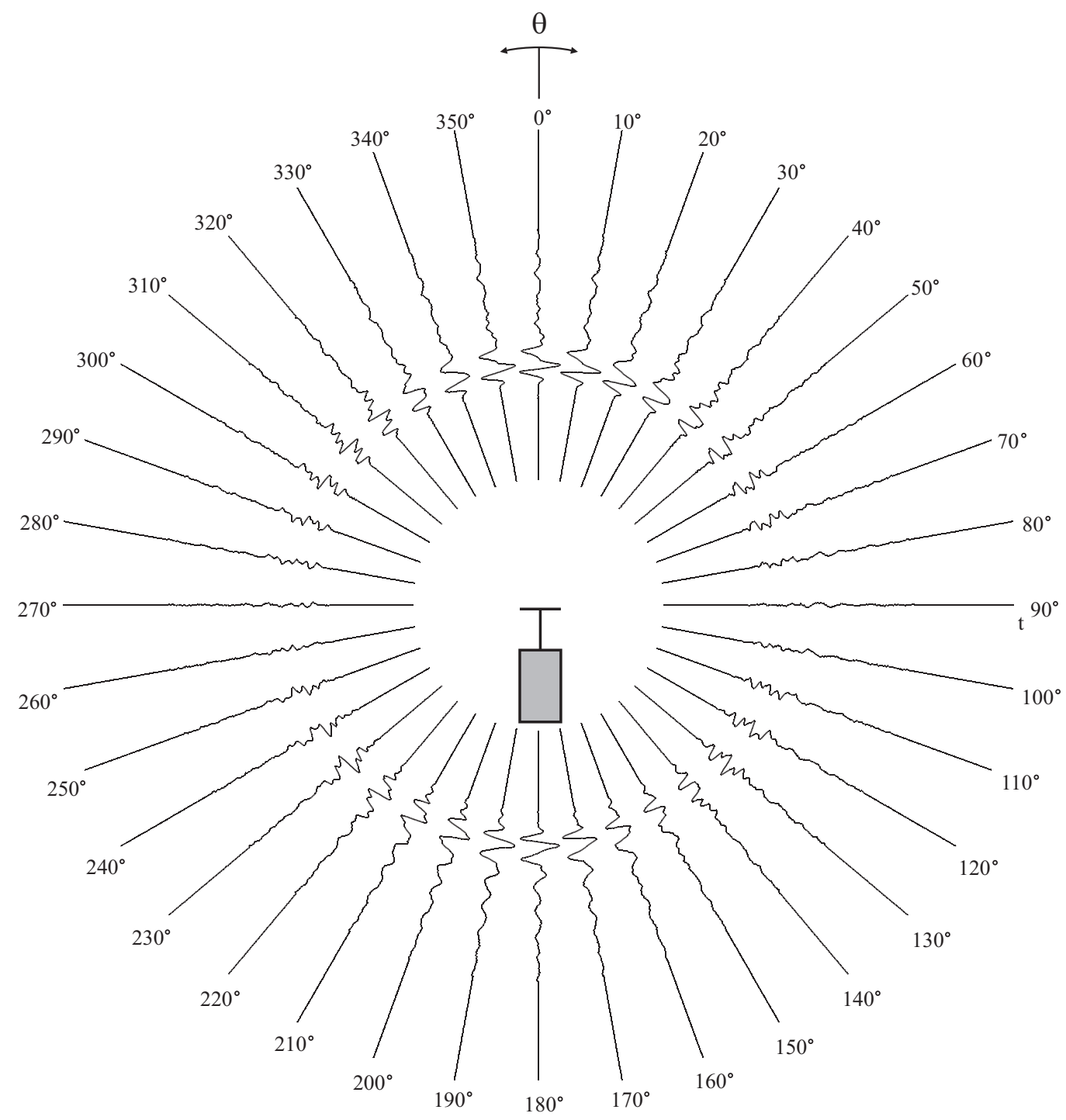

Figure 178: Time-domain pattern of the measured voltage at the probe along the elevation plane, with an incident double differentiated Gaussian pulse (spectral peak at $3 \mathrm{GHz}$ ) transmitted by a $5 \mathrm{~cm}$ dipole fed with the double-y balun. 


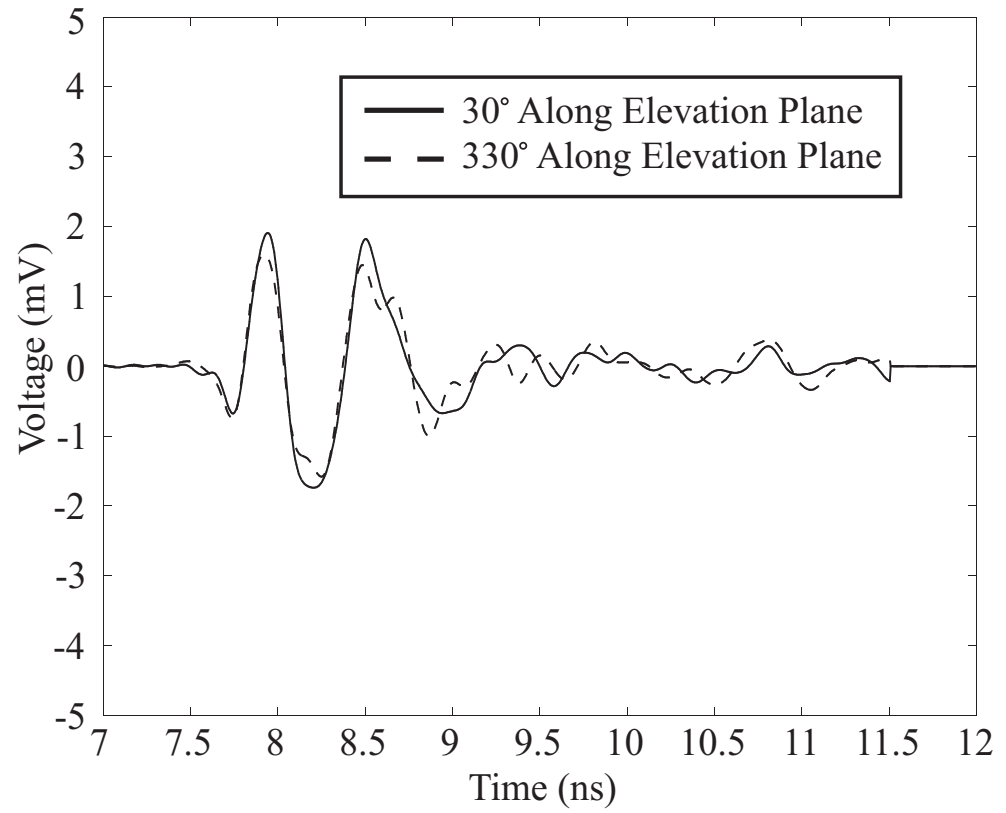

Figure 179: Plot comparing time-domain waveforms from Fig. 178 at $30^{\circ}$ and $330^{\circ}$. 


\subsubsection{Resistively Loaded V-Dipole}

Amplitude patterns were measured for a resistively loaded V-dipole fed with and without the double-y balun. Figure 180a illustrates the V-dipole fed without the double-y balun. The center conductor of a $50 \Omega$ coaxial line was soldered to one arm of the antenna. The other arm of the antenna was soldered to the outer conductor of the coaxial line. An expanded view of the feed is illustrated in Fig. 180b. Figure 181a illustrates the resistively loaded V-dipole fed with the double-y balun (80 mil stub length) designed in Section 2.7. Figure 181b illustrates an expanded view of the feed.

Figure 182 illustrates a top view of the experimental setup used for conducting pattern measurements of a resistively loaded V-dipole, fed with and without the double-y balun. A resistive V-dipole, fed with the 80 mil double-y balun designed in Section 2.7, was used as the stationary RX probe antenna. The coaxial line feeding the probe antenna was connected to port 2 of the network analyzer. The antenna under test (AUT), a resistively loaded V-dipole fed with and without the double-y balun, was fed with the laser receiver (LR) unit and mounted on the rotary positioner. The AUT was rotated about the axis of the positioner to obtain the E-plane pattern.

The measured amplitude pattern along the E-plane, at $1 \mathrm{GHz}$, for the resistive V-dipole fed without the double-y balun, is illustrated in Fig. 183a. The measured patterns in this section are compared with numerical patterns computed via Eiger (method of moments code developed at Lawrence Livermore Labs) in [30]. The numerical patterns model a balanced resistive V-dipole without the LR unit used in the experimental work. It is seen that the normalized pattern of the V-dipole fed without a balun is significantly distorted compared to the pattern generated numerically. The direction of maximum radiation is at $300^{\circ}$ instead of $0^{\circ}$. A $14 \mathrm{~dB}$ null is observed in the measured pattern at $330^{\circ}$. Sharp side lobes are also present in the measured pattern between $90^{\circ}$ and $270^{\circ}$. Figure $183 \mathrm{~b}$ illustrates the measured pattern of the 
V-dipole fed with the double-y balun (illustrated in Fig. 181). Significant improvement is seen in the measured pattern of the V-dipole fed with the double-y balun. The measured pattern agrees more closely with that obtained numerically. The deep null at $330^{\circ}$ is significantly reduced when fed with the double-y balun. Some discrepancy can be expected between the measured and numerical patterns, since the laser receiver unit used in the measurements was not included in the numerical model.

The measured pattern for the V-dipole fed without the double-y balun, at $2 \mathrm{GHz}$, is illustrated in Fig. 184a. A deep null is observed between $60^{\circ}$ and $90^{\circ}$. Additional side lobes are present between $60^{\circ}$ and $300^{\circ}$. The deep null between $60^{\circ}$ and $90^{\circ}$ is not seen in the pattern illustrated in Fig. 184b, for the V-dipole fed with the double-y balun. The discrepancy between the measured and numerical patterns near $45^{\circ}$ and $315^{\circ}$ is believed to result from either an offset in alignment between the AUT and the probe antenna or small rippling of the arms of the dipole when packaged between two pieces of polystyrene foam.

Measured patterns for the resistive V-dipole fed with and without the double-y balun, at $3 \mathrm{GHz}$, are illustrated in Fig. 185. A sharp null is observed in the measured pattern at $60^{\circ}$ and additional side lobes are observed near $300^{\circ}$. The pattern at $3 \mathrm{GHz}$ for the V-dipole fed with the double-y balun is illustrated in Fig. 184b; the measured pattern does not exhibit the sharp null and the additional side lobes at $300^{\circ}$. Comparing the balanced and unbalanced cases at $2 \mathrm{GHz}$, there is little difference between both cases at angles between $-30^{\circ}$ and $30^{\circ}$. However, this is the unpredictable nature of an unbalanced feed; it was shown in Section 3.1 that the strength of the common-mode current induced along the feedline of an unbalanced dipole varied with feedline length. Hence, at some frequencies, the effects of an unbalanced feed are mitigated.

The effects of an unbalanced feed are more clearly seen at $4 \mathrm{GHz}$, as illustrated in Fig. 186. Figure 186a illustrates the normalized pattern for the V-dipole fed without 
a balun. Side lobes are present in the measured pattern from $60^{\circ}$ to $300^{\circ}$. Deep nulls are present between $30^{\circ}$ and $60^{\circ}$ and at $330^{\circ}$. The measured pattern for the V-dipole fed with the double-y balun is illustrated in Fig. 186b. This pattern agrees closely with the pattern generated numerically for a balanced V-dipole without the LR unit. Discrepancies in the measured and numerical patterns could arise from the doubley balun not being a perfect balun, offset in the alignment of the AUT and probe antenna, and the exclusion of the LR unit in the numerical model of the V-dipole.

The patterns of the resistive V-dipole, at $5 \mathrm{GHz}$, are illustrated in Fig. 187. Figure 187a illustrates the normalized pattern for the V-dipole fed directly with a coaxial line. As was the case at $4 \mathrm{GHz}$, the pattern exhibits deep nulls and multiple side lobes between $60^{\circ}$ and $300^{\circ}$. Improvement in the measured pattern is clearly seen when the resistive V-dipole is fed with the double-y balun, as illustrated in Fig. 187b. This pattern agrees closely with the numerical pattern from $-90^{\circ}$ to $90^{\circ}$. Discrepancy from $90^{\circ}$ to $270^{\circ}$ is expected, since the numerical model does not include the LR unit.

Figure 188 illustrates the measured time-domain pattern of the received voltage by the RX V-dipole, with the TX V-dipole fed without a balun. The directional nature of the V-dipole is seen from the time-domain pattern. The pattern also clearly illustrate asymmetry resulting from an unbalanced feed. The unbalanced arms cause radiation to appear at angles between $290^{\circ}$ and $315^{\circ}$. Figure 189 illustrates the measured time-domain pattern of the resistively loaded V-dipole fed with the double-y balun. The symmetry in the pattern is clearly improved when compared with Fig. 188. To observe the improvement in the symmetry, The time-domain plots of the V-dipole fed with and without the double-y balun, at $0^{\circ}$, are compared in Fig. 191. Small reflections trailing the main pulse are observed for both cases. These reflections are likely caused by ringing of the dipole arms as well as reflections from the balun junction (the ripples are more pronounced for the case with the balun). From 12.5 ns to $15 \mathrm{~ns}$, the time-domain data for the $\mathrm{V}$-dipole fed without a balun is seen to 
contain more reflections than the case with the balun. Reflection at $15 \mathrm{~ns}$ is observed in both cases; this reflection is likely caused by clutter, since the feed structures for the V-dipole fed with and without the double-y balun are different. Therefore it is unlikely for a feedline reflection to occur for both cases at $15 \mathrm{~ns}$.

Normalized patterns for the V-dipole were illustrated in Figs. 183-187. These patterns illustrated the improvement in the patterns of the V-dipole when fed with the double-y balun. However, these plots tell us little regarding the gain of the antenna with the use of the double-y balun. Based on the pattern measurements conducted with the V-dipole, fed with and without the balun, a relative gain is defined. This gain is given by

$$
G_{r e l}=10 \log _{10}\left(\frac{V_{d y}}{V_{n b}}\right)^{2},
$$

where $V_{d y}$ is the measured voltage by the RX antenna in Fig. 182, with the TX antenna fed with the double-y balun, and $V_{n b}$ is the measured voltage by the RX antenna in Fig. 182, with the TX antenna fed without the double-y balun. Figure 192 illustrates the relative gain, as defined in Equation 44, with both antennas facing each other. A relative gain between 0 to $3.75 \mathrm{~dB}$ is obtained, with the use of the double-y balun, from $1 \mathrm{GHz}$ to $8 \mathrm{GHz}$. When computing the relative gain, the clutter in Fig. 191 near 15 ns was time-gated out. This resulted in a smoother gain plot in Fig. 192.

The measured patterns of a resistively loaded V-dipole illustrate the effects of an unbalanced feed. While there was little difference at some frequencies between the patterns of a V-dipole fed with and without the double-y balun, the V-dipole without a balun exhibited deep nulls and additional side lobes at higher frequencies. These deep nulls and additional side lobes were not observed for the V-dipole fed with the double-y balun. Hence, improvement is seen in the pattern of the resistively V-dipole fed with the double-y balun. 


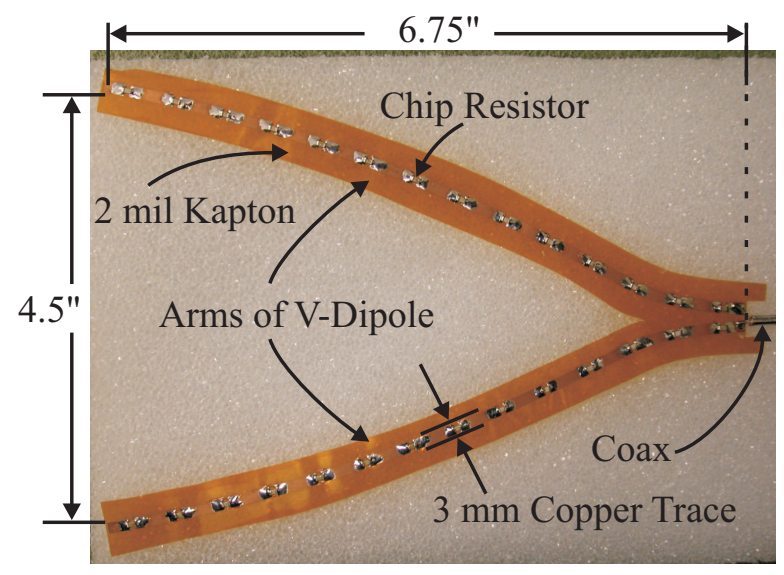

(a)

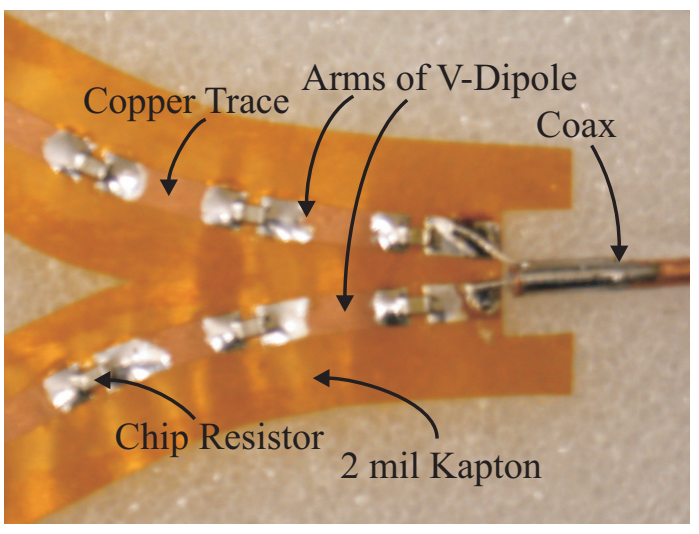

(b)

Figure 180: Illustration of resistively loaded V-dipole fed (a) without a balun and (b) expanded view of feedpoint. 


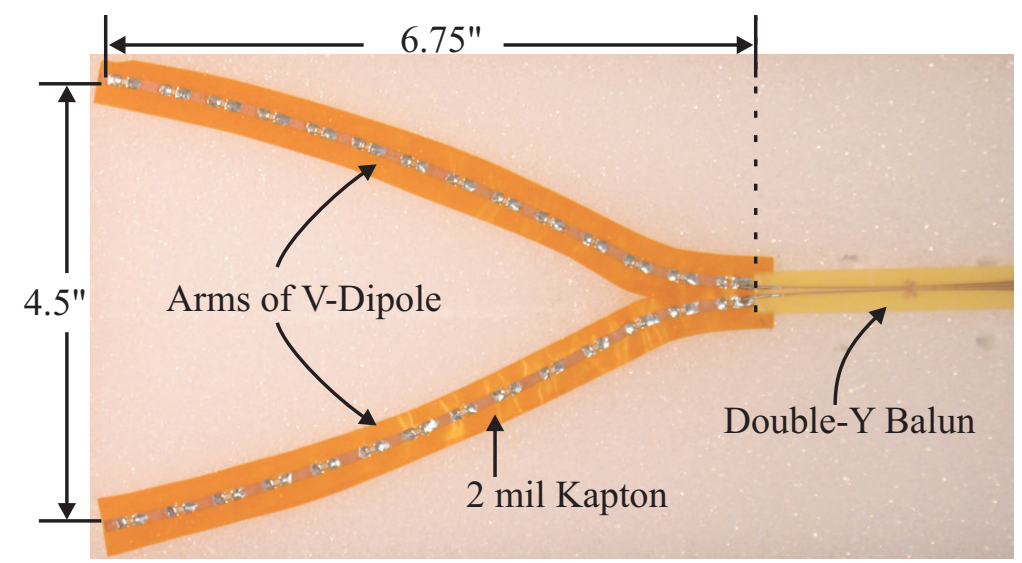

(a)

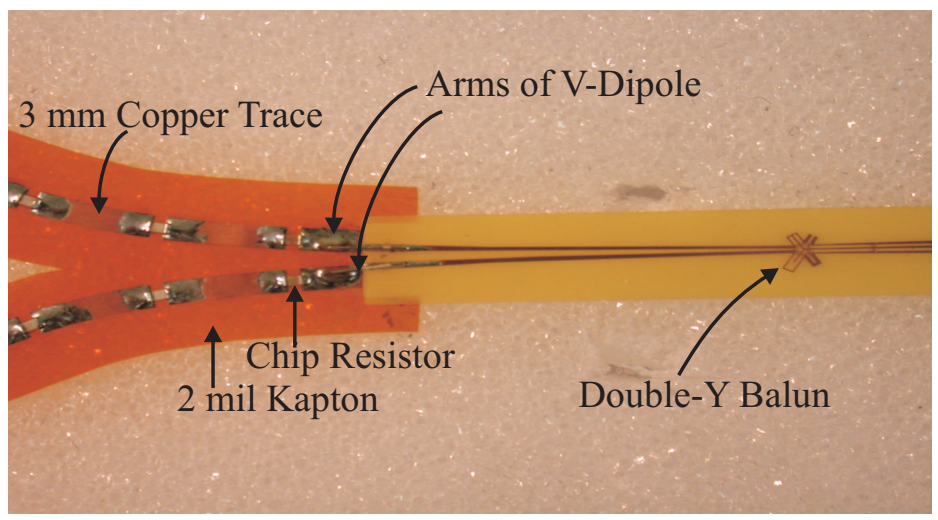

(b)

Figure 181: Illustration of resistively loaded V-dipole fed (a) with the double-y balun and (b) expanded view of feedpoint.

\section{Top View of Measurement Setup}
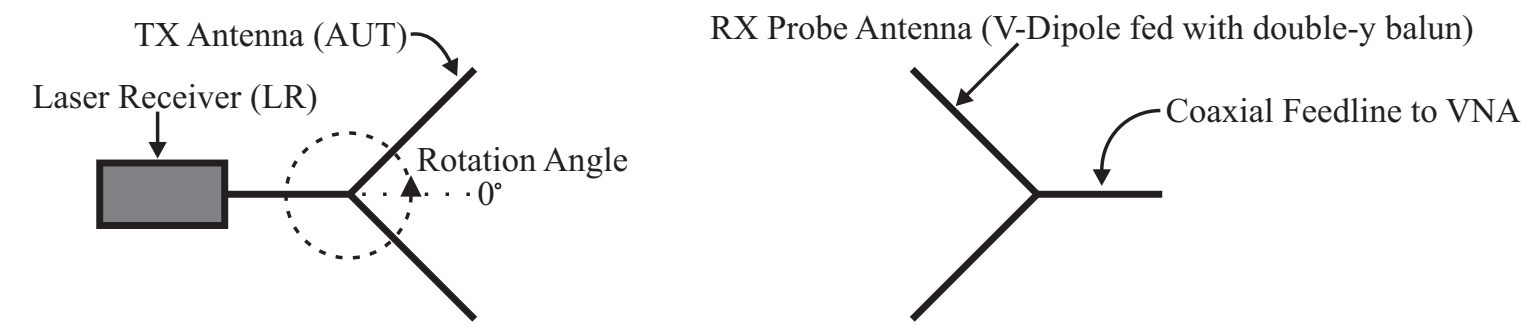

Figure 182: Illustration of experimental setup for measuring the antenna pattern along the E-plane of a resistively loaded V-dipole, fed with and without the double-y balun. 


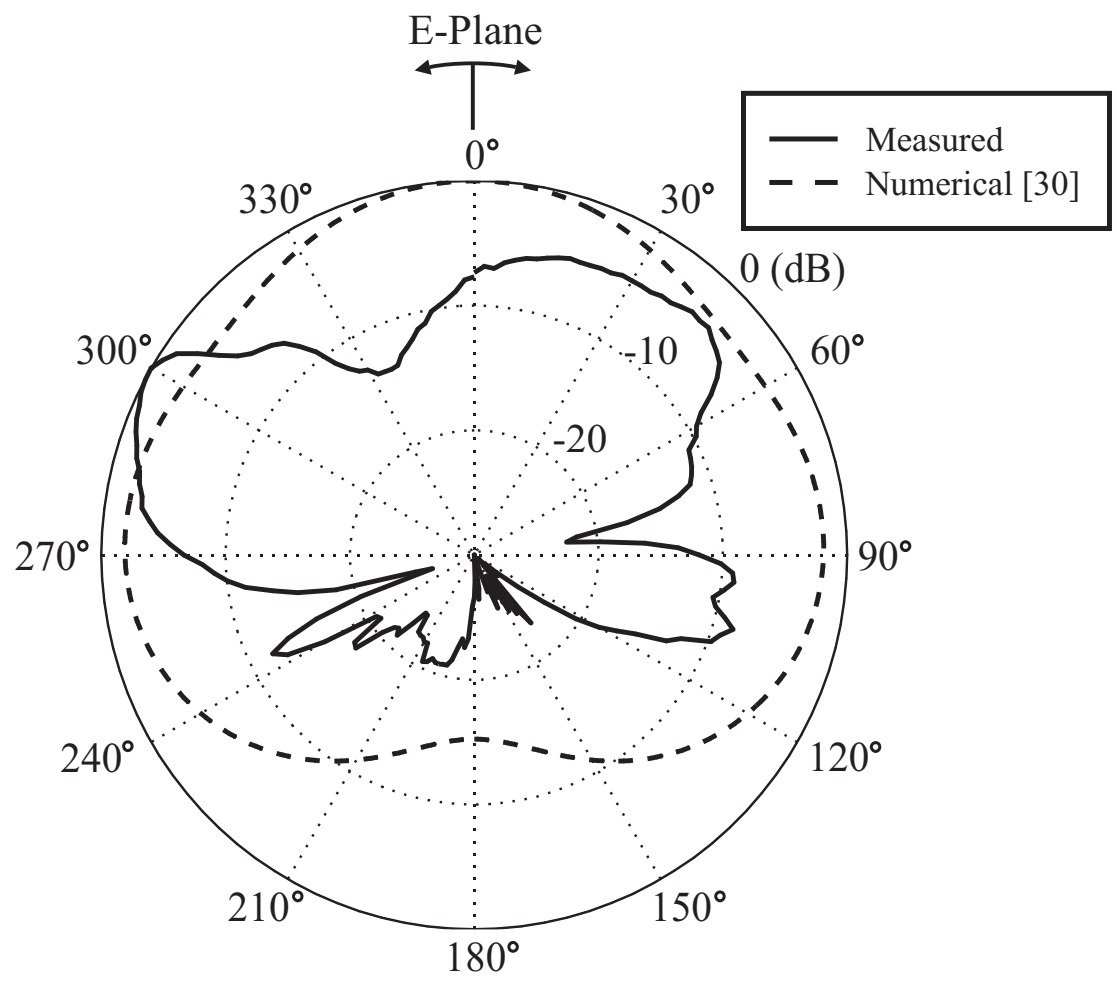

(a)

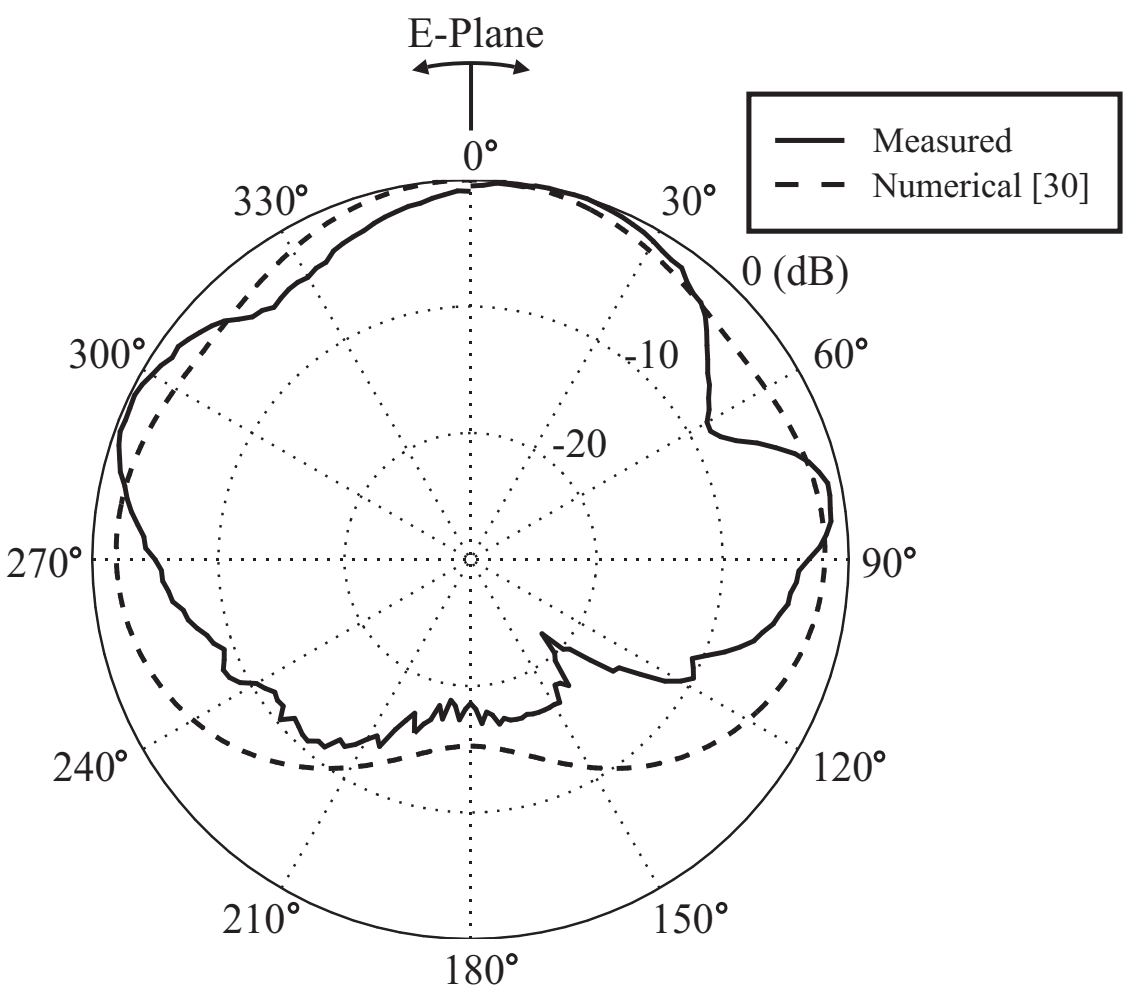

(b)

Figure 183: Measured vs. numerical normalized amplitude pattern at $1 \mathrm{GHz}$ for resistively loaded V-dipole fed (a) without a balun and (b) with the double-y balun. Numerical patterns model a balanced V-dipole. 


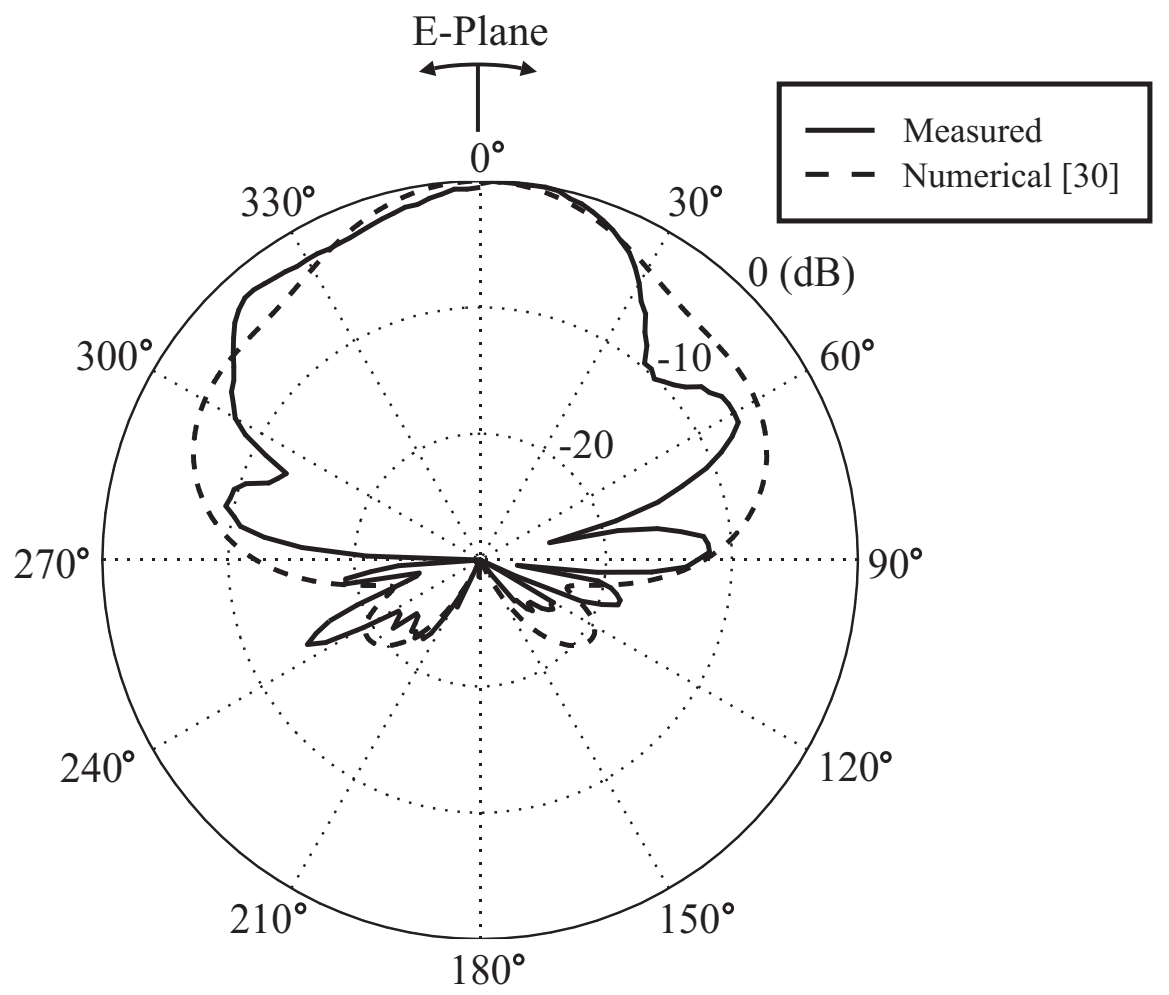

(a)

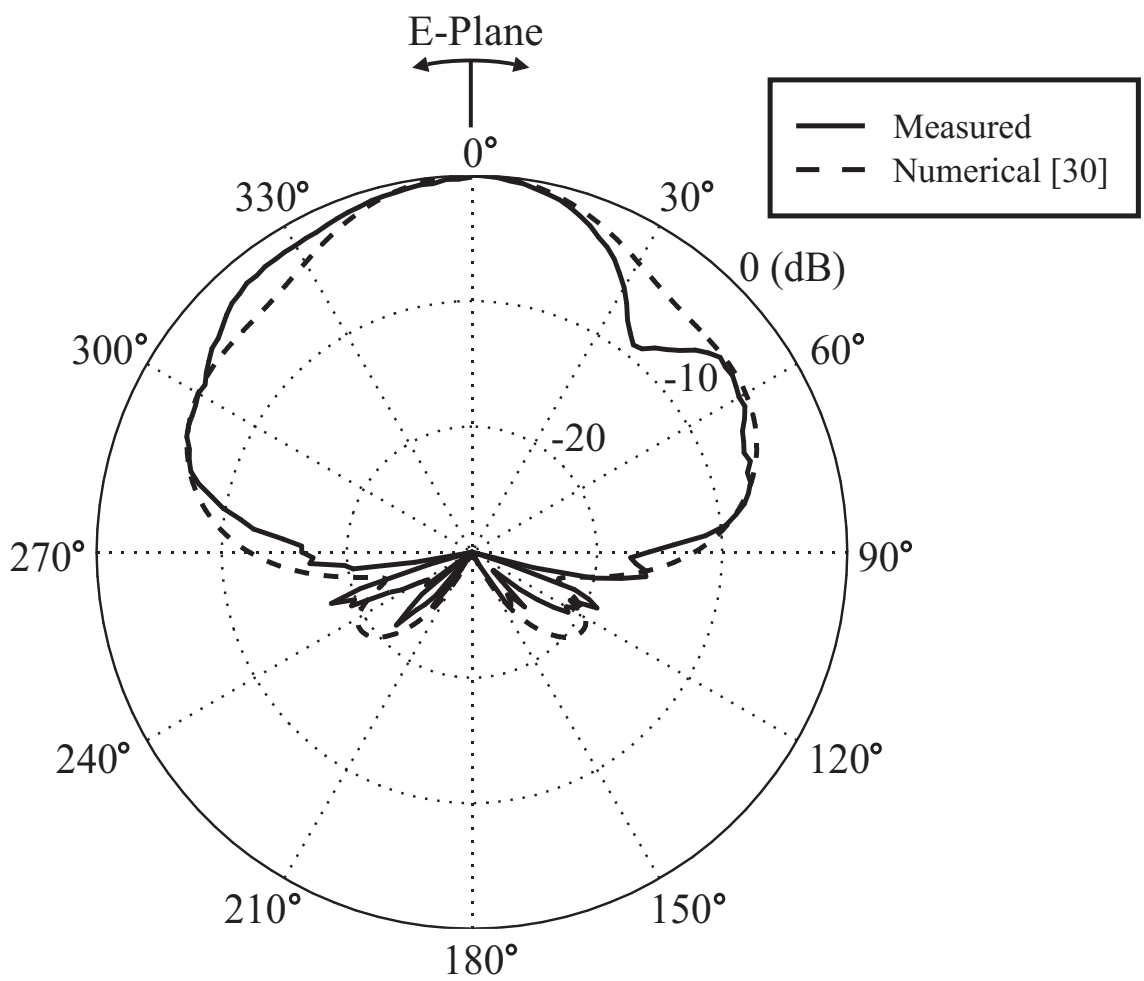

(b)

Figure 184: Measured vs. numerical normalized amplitude pattern at $2 \mathrm{GHz}$ for resistively loaded V-dipole fed (a) without a balun and (b) with the double-y balun. Numerical patterns model a balanced V-dipole. 


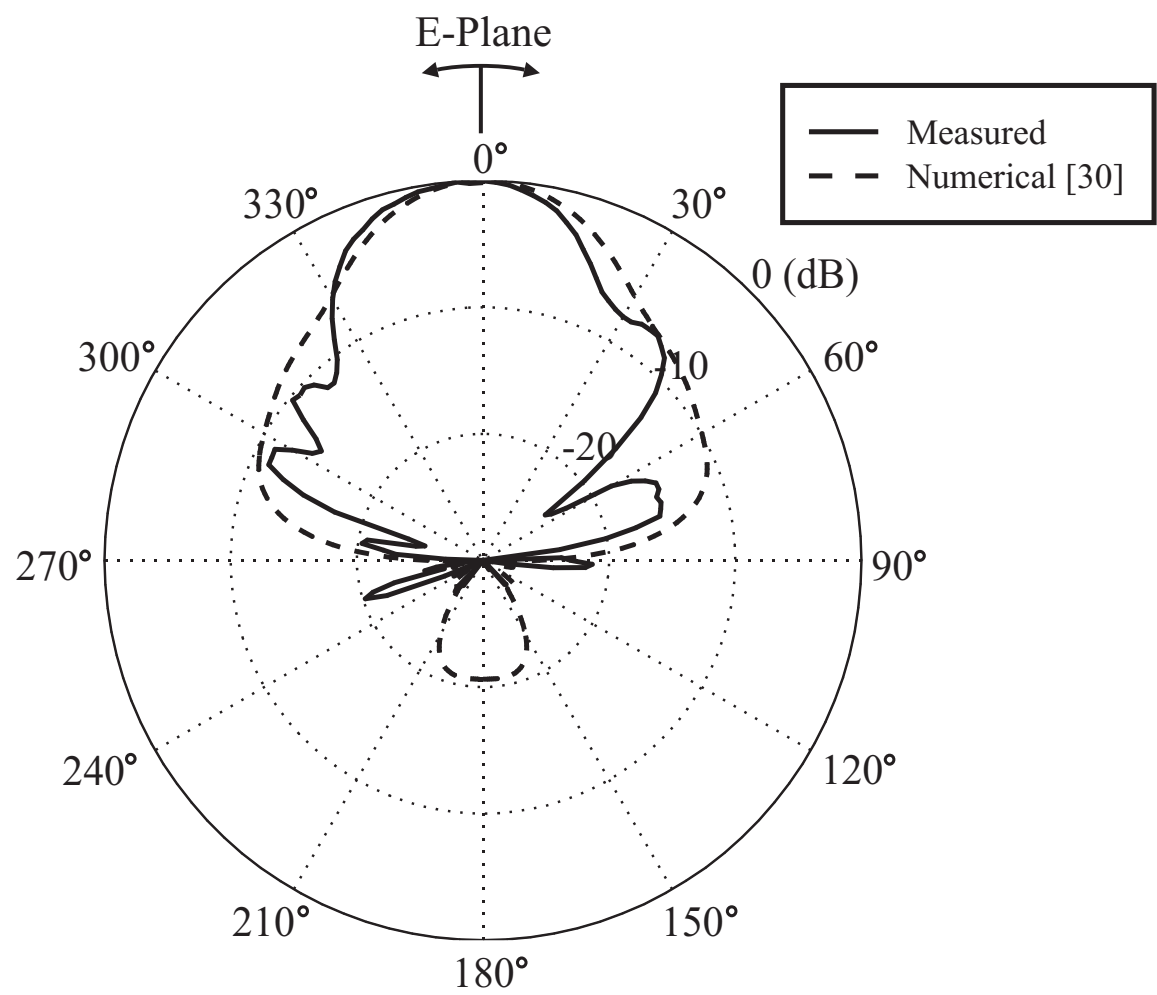

(a)

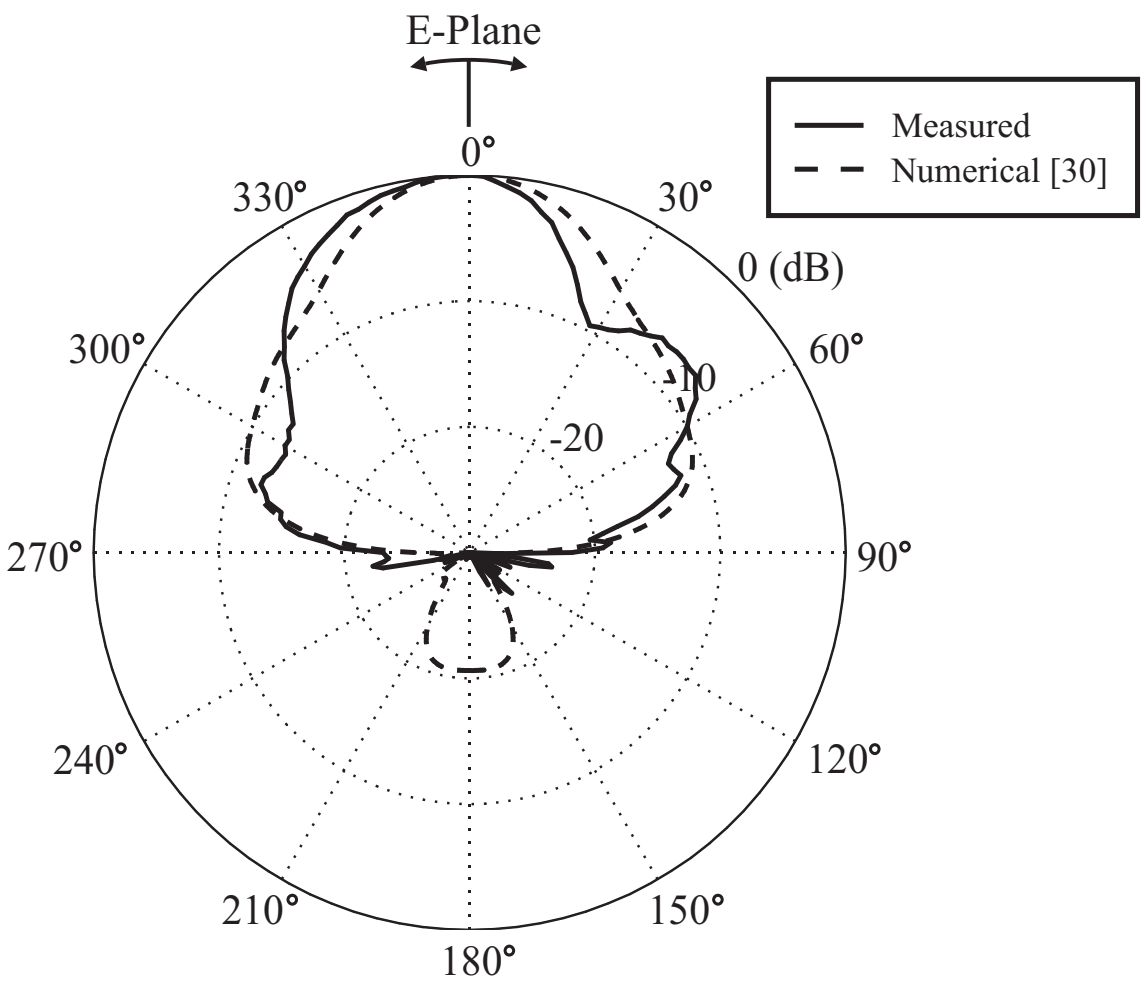

(b)

Figure 185: Measured vs. numerical normalized amplitude pattern at $3 \mathrm{GHz}$ for resistively loaded V-dipole fed (a) without a balun and (b) with the double-y balun. Numerical patterns model a balanced V-dipole. 


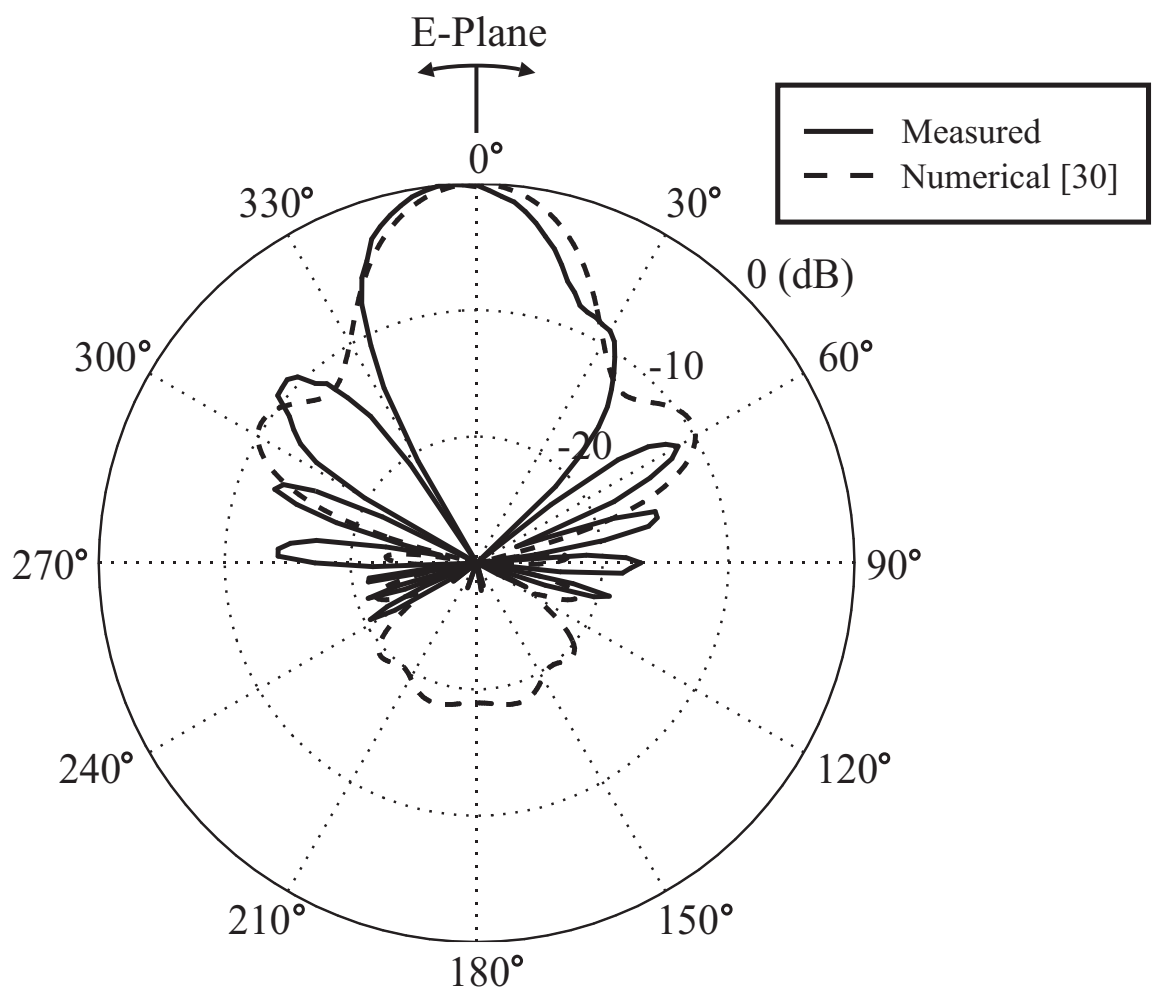

(a)

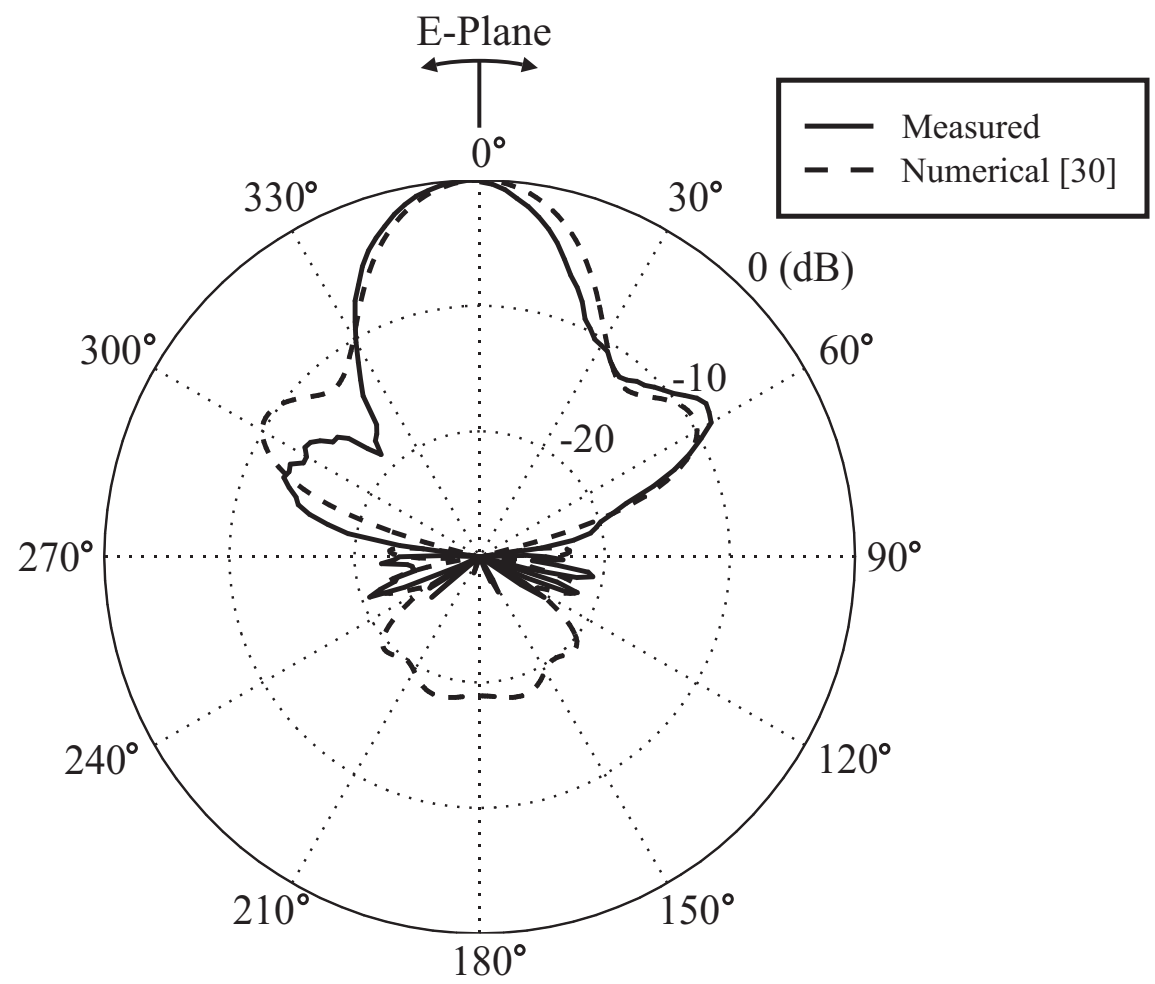

(b)

Figure 186: Measured vs. numerical normalized amplitude pattern at $4 \mathrm{GHz}$ for resistively loaded V-dipole fed (a) without a balun and (b) with the double-y balun. Numerical patterns model a balanced V-dipole. 


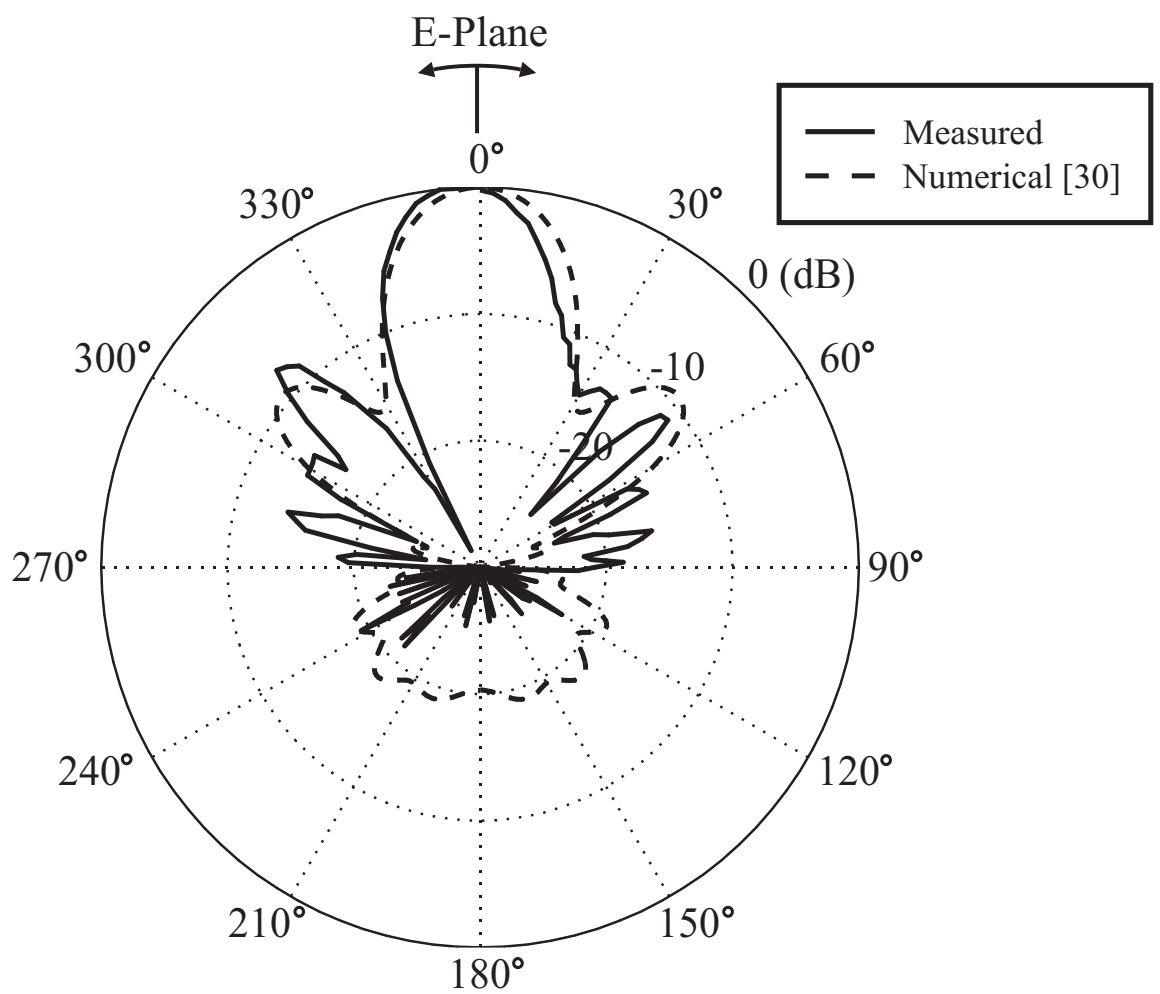

(a)

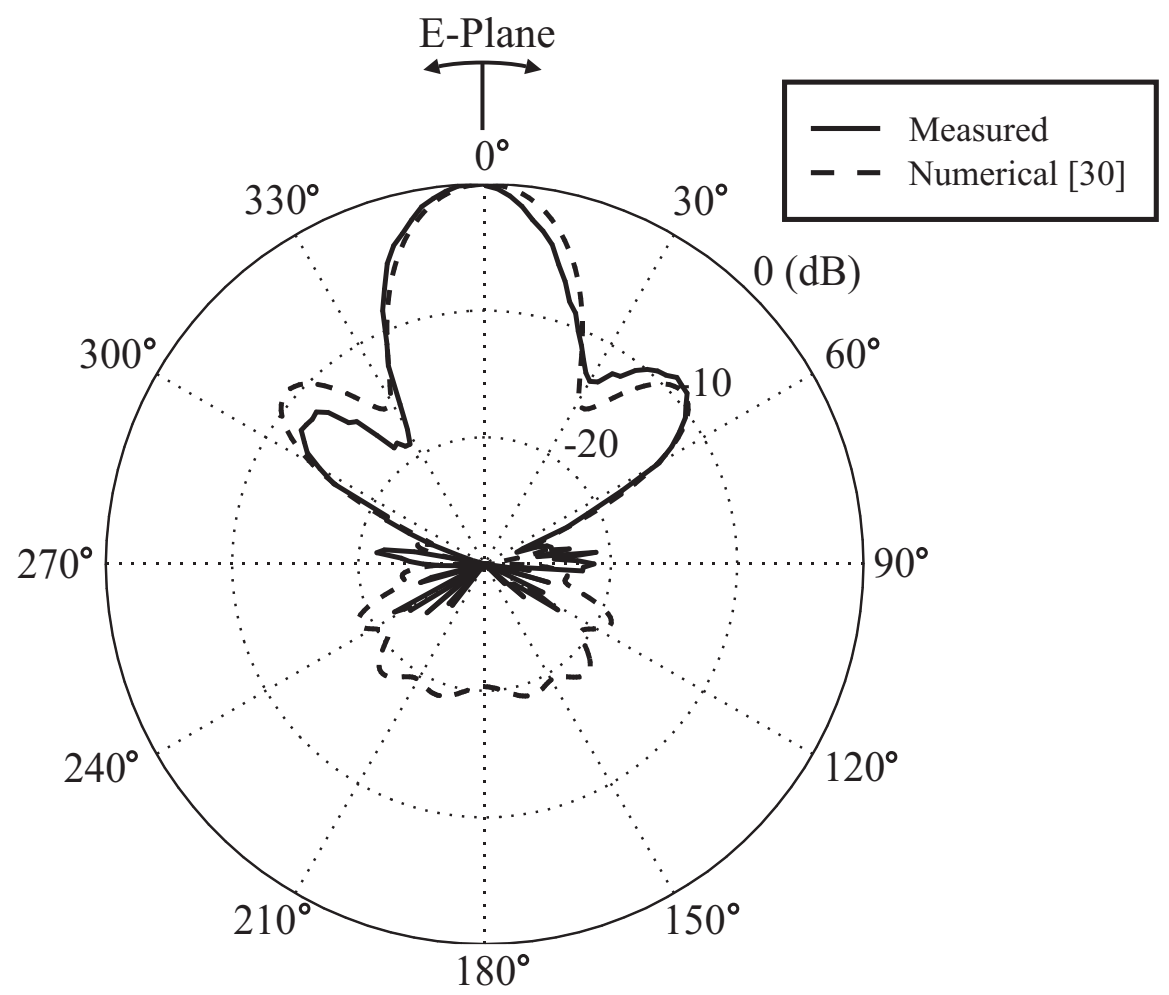

(b)

Figure 187: Measured vs. numerical normalized amplitude pattern at $5 \mathrm{GHz}$ for resistively loaded V-dipole fed (a) without a balun and (b) with the double-y balun. Numerical patterns model a balanced V-dipole. 


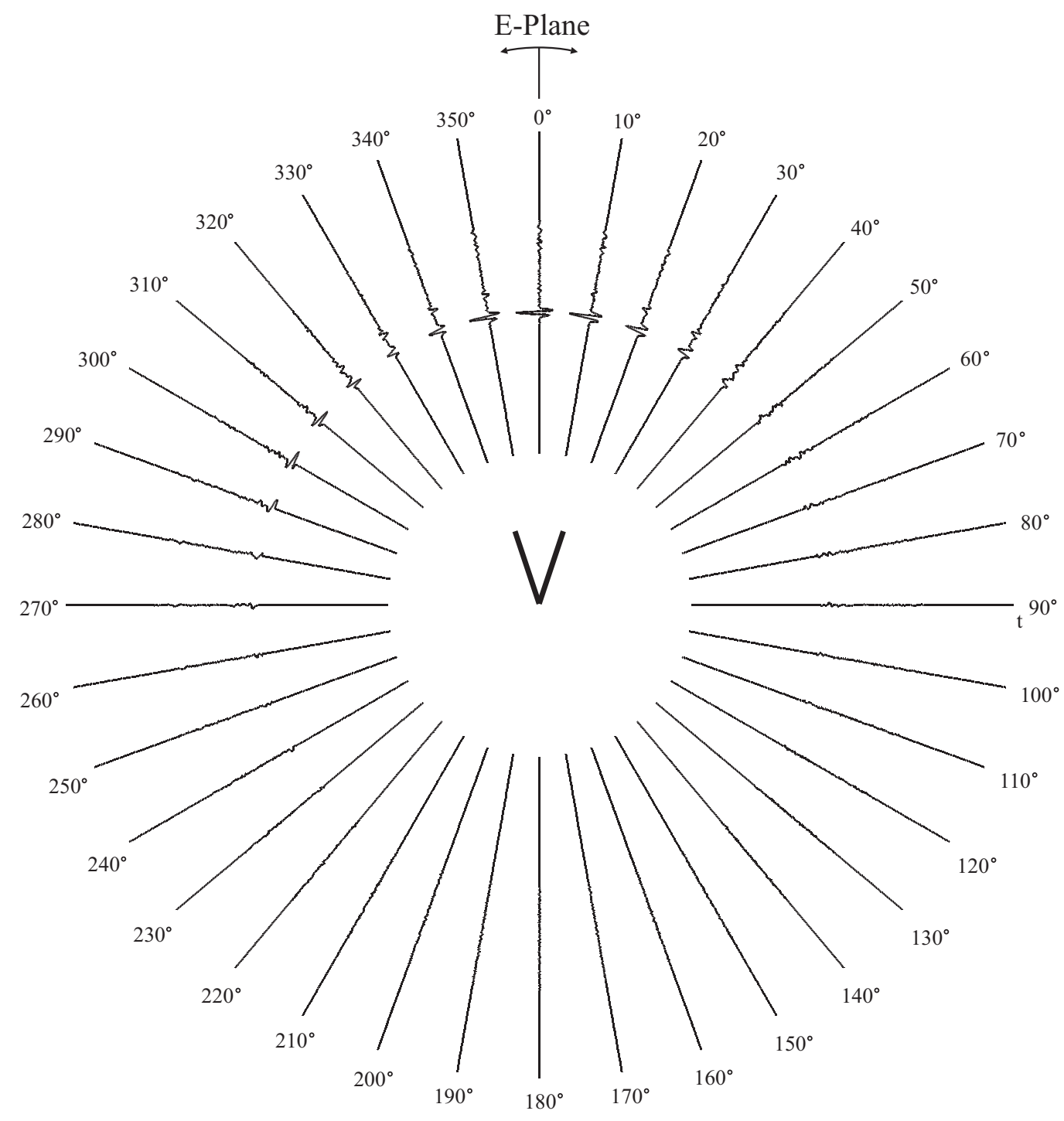

Figure 188: Measured time-domain pattern of received voltage by the RX V-dipole in Fig. 182, with the transmitting TX V-dipole fed without the double-y balun (illustrated in Fig. 180). The TX V-dipole was excited with a $1 \mathrm{~V}$-peak differentiated Gaussian pulse with spectral peak at $3 \mathrm{GHz}$. 


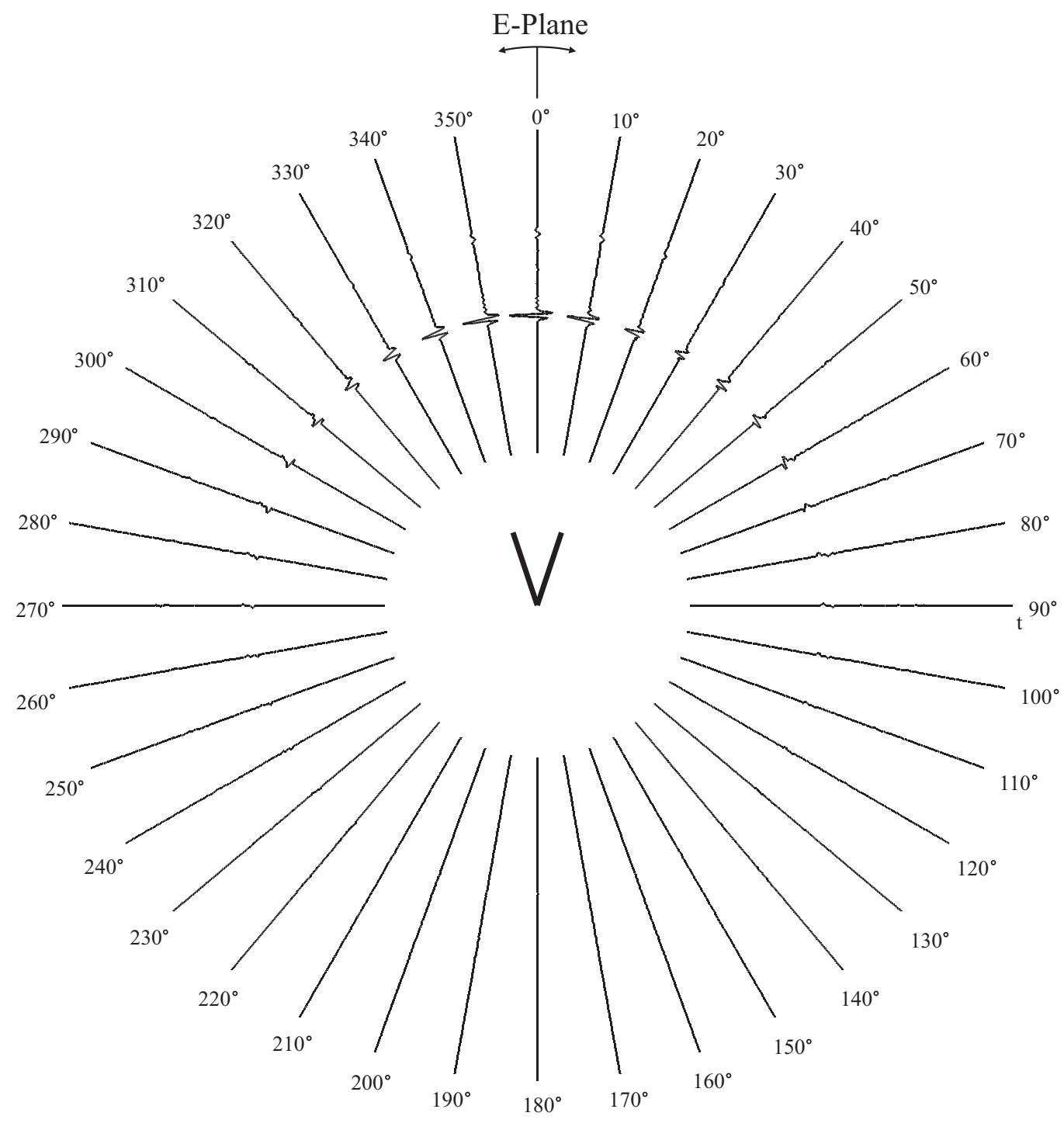

Figure 189: Measured time-domain pattern of received voltage by the RX V-dipole in Fig. 182, with the transmitting TX V-dipole fed with the double-y balun (illustrated in Fig. 181). The TX V-dipole was excited with a $1 \mathrm{~V}$-peak differentiated Gaussian pulse with spectral peak at $3 \mathrm{GHz}$. 


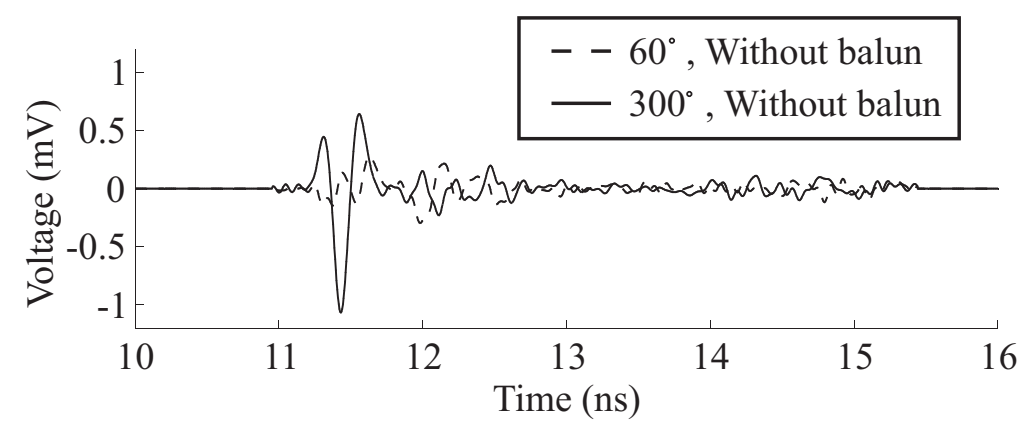

(a)

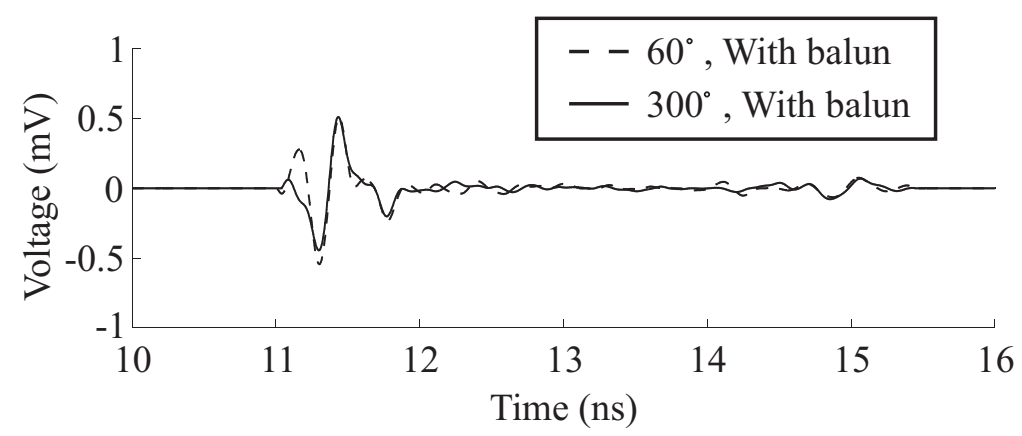

(b)

Figure 190: Plots comparing time-domain waveforms in Fig. 189 of the received voltage by the RX V-dipole at $60^{\circ}$ and $300^{\circ}$, with the TX V-dipole fed (a) without a balun and (b) with the double-y balun. 


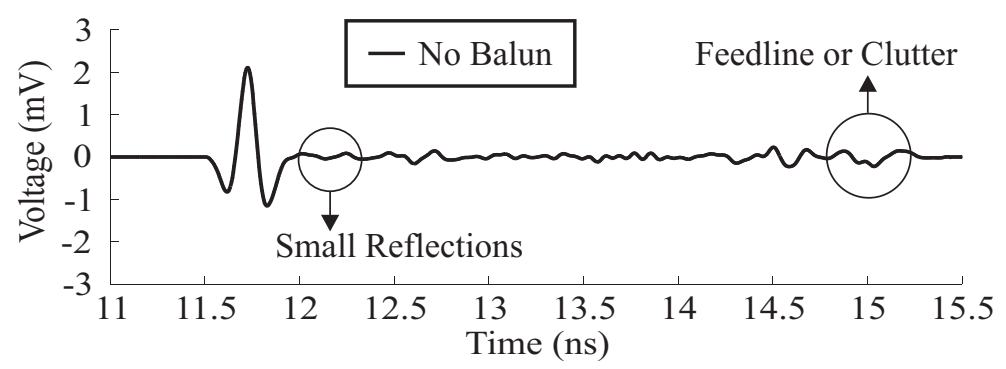

(a)

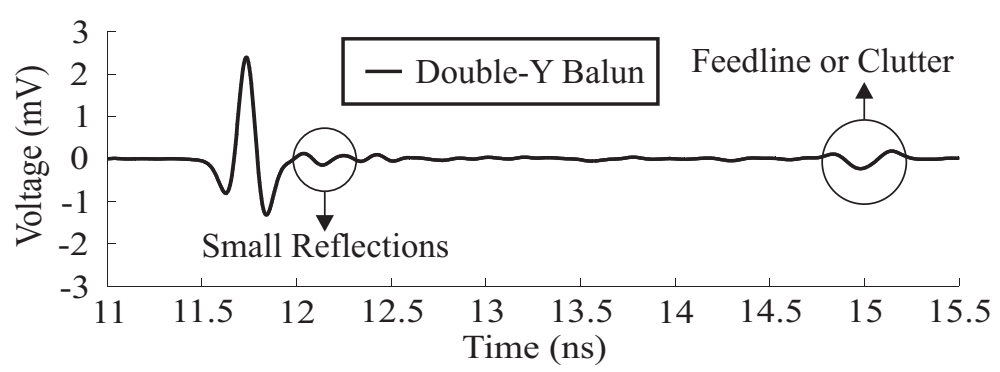

(b)

Figure 191: Comparison of the time-domain received voltage by the RX V-dipole (in Fig. 182) at $0^{\circ}$, with the TX V-dipole fed (a) without and (b) with the double-y balun. The TX V-dipole was excited with a 1 V-peak differentiated Gaussian pulse with spectral peak at $3 \mathrm{GHz}$.

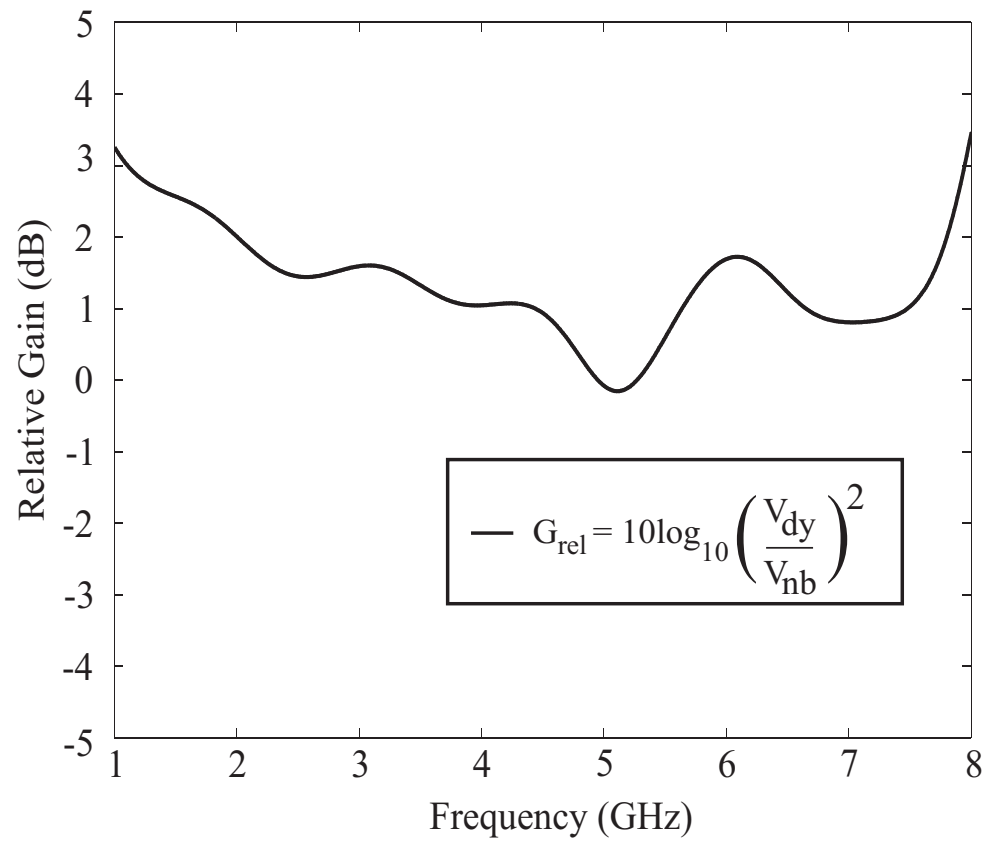

Figure 192: Plot of relative gain, as defined in Equation 44, with RX and TX $\mathrm{V}$-dipoles facing each other at $0^{\circ}$. 


\section{CHAPTER VI}

\section{CONCLUSION}

In Chapter 1, the concept of a balanced antenna feed was introduced, and the need for a balun when feeding symmetric antennas was explained. Symmetric antennas such as the dipole and the resistively loaded V-dipole require a balanced feed to radiate properly. A balanced antenna feed is defined as having only differential-mode currents, that is, currents equal in magnitude and opposite in phase. Theoretical patterns for antennas such as the dipole are computed by assuming differential-mode currents along the arms of the antenna. The coaxial line is often used to carry highfrequency signals. The coaxial line can also support common-mode current along its conductors. If a dipole is fed directly with a coaxial line, the asymmetry between the arms of the dipole and the coaxial line induces a common-mode current along the conductors of the coaxial line, thus causing the coaxial feedline to radiate. Hence, in order to transition from a symmetric antenna requiring a balanced feed, to an unbalanced structure (e.g. coaxial line), a balun is required. In this research, the double-y balun was investigated for use with pulsed antennas, specifically for use with a resistively loaded V-dipole. The double-y balun of interest in this research transitions from a three conductor coplanar waveguide (CPW) to a two conductor coplanar strip (CPS) line.

In Chapter 2, design charts were generated for CPW and CPS sections over FR4 substrate. For the CPW, it was shown that decreasing the substrate thickness or decreasing the outer conductor width increases the characteristic impedance of the line. Similarly, increasing the gap width also increases the characteristic impedance of the CPW line. Impedance curves were generated for the CPW over FR4, RT/6006, 
and Alumina. From these curves, it was seen that the impedance of the CPW decreases with increasing substrate permittivity. It was shown from CPS design charts that the characteristic impedance decreases with increasing substrate thickness, decreasing gap width, or increasing conductor width. As was the case with CPW lines, the characteristic impedance of CPS lines decreases with increasing substrate thickness. The double-y balun requires that CPW and CPS stubs have equal characteristic impedances as well as equal electrical lengths in order to exhibit an all-pass behavior. However, in this research, this balun is required to transition from a $50 \Omega$ coaxial line to a $200 \Omega$ resistively loaded V-dipole. From the CPW and CPS design charts, it was shown that it is difficult to achieve $50 \Omega$ CPS lines and $200 \Omega$ CPW lines. Hence, impedance tapers were designed to transition from the coaxial line to the balun, and to transition from the balun to the antenna.

The double-y balun was modeled as a two-port device using two commercial electromagnetic solvers: Momentum, a method of moments code, and HFSS, a finite element based code. To reduce computational time, the double-y balun was modeled without impedance tapers in both simulators. The resulting s-parameters from both simulators showed good agreement. Resonances, limiting the bandwidth of operation, were observed in the simulated $s_{21}$ data. In this research work, the effectiveness of multiple CPW bridges in suppressing the CPW resonance was investigated. It was shown that the insertion loss due to the CPW resonance can be reduced via the use of multiple CPW bridges. It was also shown that the additional CPW bridges cannot be placed arbitrarily close to the junction. When dealing with CPW and CPS lines, various combinations of conductor and gap widths can be synthesized for a given characteristic impedance. Hence, double-y baluns with varying aspect ratios can be designed for a given CPW/CPS impedance. To determine the effect of the aspect ratio on the balun's performance, two double-y baluns with varying aspect ratios were modeled in Momentum and the resulting $\mathrm{s}_{21}$ was computed. There was little 
difference in the resulting $\mathrm{s}_{21}$ data for both cases, hence the aspect ratio of the balun does not affect the s-parameters significantly. Further experimental work needs to be conducted to determine whether the aspect ratio of the balun affects the balancing behavior of the balun. Ideally, the electrical lengths of the open and shorted CPW and CPS stubs should be equal for the double-y balun to exhibit an all-pass behavior. Therefore, the physical lengths of the stubs must be compensated for unequal dispersion between CPW and CPS lines and fringing effects of open and shorted stubs. A double-y balun was designed with stub lengths compensated for fringing effects. The balun was simulated using Momentum; compensating the stub lengths had little effect on the resulting $\mathrm{s}_{21}$ obtained numerically. While the balun cannot be completely characterized by looking at $s_{21}$, it is seen that varying the aspect ratio of the balun, or adjusting the lengths of the stubs to account for fringing effects, has little effect on the balun's insertion loss. Using the above results, a double-y balun was designed for manufacturing over 20 mil FR4 substrate. The balun was designed to transition from a $50 \Omega$ coaxial line to a $200 \Omega$ resistive V-dipole. Exponential impedance tapers were designed to achieve the necessary impedance transformations.

In Chapter 3, a dipole, along with the outer conductor of a coaxial line, was modeled using Numerical Electromagnetic Code (NEC). Modeling a dipole and the outer conductor of a coaxial line serves two purposes: (1) provide a better insight into the effects of an unbalanced dipole feed and (2) generate patterns that can be used as a basis of comparison for measured patterns. Using the NEC model, currents along the arms and feedline of a balanced and an unbalanced dipole were studied. When the arms of the dipole were unbalanced, a common-mode current was induced along the feedline.

Normalized amplitude patterns were generated for a balanced dipole and an unbalanced dipole along the azimuth and elevation planes. It was shown that it is difficult to discern between the azimuth patterns, at $\lambda / 2$, of a balanced dipole and an 
unbalanced dipole. The pattern of the unbalanced dipole was slightly rotated. Thus, to be able to discern between the two patterns, an accurate measurement system is required. It was also shown that the azimuth patterns of an unbalanced dipole at $3 \lambda / 2$ and $5 \lambda / 2$ were significantly different from patterns of a balanced dipole. Hence, the effect of the unbalanced feed on the azimuth pattern depends on the current distribution along the arms of the dipole. Elevation patterns were significantly affected at all frequencies for the unbalanced dipole. However, it was shown that the pattern along the elevation plane varies with feedline length; changing the length of the feedline alters the current distribution along the feedline. The patterns of an unbalanced dipole with a finite length feedline were compared with patterns of an unbalanced dipole with an infinite length feedline; the infinite length feedline was approximated numerically by loading the end segments of the feedline with a Wu-King profile. The resulting patterns illustrated that varying the length of the feedline had little effect on the pattern along the azimuth plane, however, the pattern along the elevation plane changed significantly.

The NEC model of a dipole and the outer conductor of a coaxial line was modified to conduct time-domain analysis of a balanced dipole and an unbalanced dipole. Additional reflections from the feedline were shown to be present in the case of an unbalanced dipole feed. The effect of time-gating the reflected pulses on the patterns along the azimuth and elevation planes was also studied. It was shown that timegating the reflections had little effect on the pattern along the azimuth plane, however, removing the reflections did alter the patterns along the elevation plane. These results were consistent with those obtained by changing the feedline length; time-gating the reflections from the feedline is equivalent to modeling an infinite feedline. In a practical measurement system, scattering from nearby objects affects the measured antenna patterns. These reflections are reduced by conducting the measurements in an anechoic chamber or the use of absorbers. The time-domain results from NEC 
suggest that when conducting pattern measurements along the elevation plane, the minimum time window should be such that the first reflection from the feedline should be captured. That is, the first reflection from clutter should occur after the first reflection from the unbalanced feedline.

Results from the numerical model of an unbalanced dipole in Chapter 3 motivated the design of a new measurement system for conducting antenna pattern measurements. It was shown in Chapter 3 that the pattern along the elevation plane is very sensitive to the length and position of the coaxial feedline. In Chapter 4, a new measurement system was designed with the use of an optical link. A laser modulator (LM ) unit and a laser receiver (LR) unit were designed for use in the new measurement system. The laser modulator modulated light with the RF signal from the network analyzer. The modulated signal was transmitted via fiber optic cable to the battery powered LR unit, where the RF signal was demodulated and was fed to the antenna under test (AUT). Thus, with the use of an optical link, the coaxial feedline was truncated and the AUT was isolated electrically.

Experimental work was conducted in Chapter 5 to characterize the double-y balun designed in Chapter 2 via VSWR, insertion loss, and pattern measurements of antennas fed with and without the balun. Two versions of the double-y balun were manufactured, one with 80 mil CPW/CPS stubs and the other with 110 mil CPW/CPS stubs. VSWR measurements were conducted by terminating the double-y balun with two $100 \Omega$ chip resistors in series. Using two chip resistors reduces the parasitic capacitance of the chip resistors. Both baluns achieved a VSWR of less than 1.5 from $800 \mathrm{MHz}$ to $10 \mathrm{GHz}$. Insertion loss measurements were conducted with back to back double-y baluns. The double-y balun with 80 mil stubs achieved an insertion loss of less than $1.8 \mathrm{~dB}$ from $800 \mathrm{MHz}$ to $10 \mathrm{GHz}$. The double-y balun with 110 mil stubs achieved an insertion loss of less than $2.5 \mathrm{~dB}$ from $800 \mathrm{MHz}$ to $10 \mathrm{GHz}$. The effect of adding additional CPW bridges along the input CPW section was also investigated. 
Insertion loss measurements of back to back double-y balun with additional CPW bridges showed improved performance of the balun with the additional bridges. In order to obtain better insight into the performance of the double-y balun, antenna pattern measurements were conducted for dipoles fed with and without the double-y balun with 80 mil stubs.

Antenna patterns were measured along the azimuth plane for a $5 \mathrm{~cm}$ dipole fed directly with a coaxial line (without a balun), a $5 \mathrm{~cm}$ dipole fed with the double-y balun, a $5 \mathrm{~cm}$ dipole fed with the sleeve balun, a $12 \mathrm{~cm}$ dipole fed directly with a coaxial line (without a balun), and a $12 \mathrm{~cm}$ dipole fed with the double-y balun. An automated measurement system, employing a rotary positioner, was constructed to conduct accurate pattern measurements. The measured patterns were compared with patterns generated numerically using the NEC model of an unbalanced dipole discussed in Chapter 3. The measured patterns of the unbalanced dipoles agreed closely with the NEC patterns of an unbalanced dipole. Measured patterns of the dipoles fed with the baluns agreed sufficiently with the NEC patterns to illustrate the improvement obtained with the use of a balun.

Patterns were also measured for the above mentioned antennas along the elevation plane. The experimental setup used to conduct pattern measurements along the azimuth plane was modified to include the optical link designed in Chapter 4. Measured patterns of the above mentioned antennas were compared with patterns generated via the NEC model in Chapter 3, modified to include the LR unit. Measured patterns agreed sufficiently with the NEC patterns to illustrate the improvement obtained with the use of a balun.

Finally, patterns were measured along the E-plane for a resistively loaded V-dipole fed with and without the double-y balun. Measured patterns were compared with numerical patterns available in literature for the resistive V-dipole with balanced arms. The numerical model, however, does not include the LR unit. Therefore, some 
discrepancy between the measured and numerical patterns is expected. Patterns of the resistively loaded V-dipole fed without the double-y balun (fed directly with a coaxial line) exhibited additional deep nulls and side lobes. These effects were more pronounced at higher frequencies. The resistive V-dipole fed with the double-y balun agreed more closely with the patterns obtained numerically, thus illustrating the improvement in the patterns with the use of the double-y balun.

This research work investigated the double-y balun for feeding pulsed antennas such as the resistively loaded V-dipole. Design aspects of the double-y balun were investigated numerically using commercial electromagnetic simulators. Improved insight into the effects of an unbalanced dipole feed was presented via NEC models. A new experimental setup was constructed to measure patterns of dipoles fed with and without baluns. Resulting pattern measurements were compared with numerical results to illustrate the need of a balun when feeding symmetric antennas. 


\section{APPENDIX A}

\section{GENERALIZED 2-PORT S-PARAMETERS}

Figure 193 illustrates a 2-port network; each port has a source, labeled $\mathrm{E}_{s}$, and a reference impedance, labeled $\mathrm{Z}_{i}$. The scattering matrix for this 2-port network is defined as

$$
[S]=\left[\begin{array}{ll}
S_{11} & S_{12} \\
S_{21} & S_{22}
\end{array}\right]
$$

and is related to the incident and reflected wave amplitudes by

$$
\begin{aligned}
& b_{1}=S_{11} a_{1}+S_{12} a_{2} \\
& b_{2}=S_{21} a_{1}+S_{22} a_{2}
\end{aligned}
$$

where

$$
\begin{aligned}
a_{i} & =\frac{V_{i}+I_{i} Z_{i}}{2 \sqrt{\left|\operatorname{Re}\left(Z_{i}\right)\right|}} \\
b_{i} & =\frac{V_{i}-I_{i} Z_{i}^{*}}{2 \sqrt{\left|\operatorname{Re}\left(Z_{i}\right)\right|}}
\end{aligned}
$$

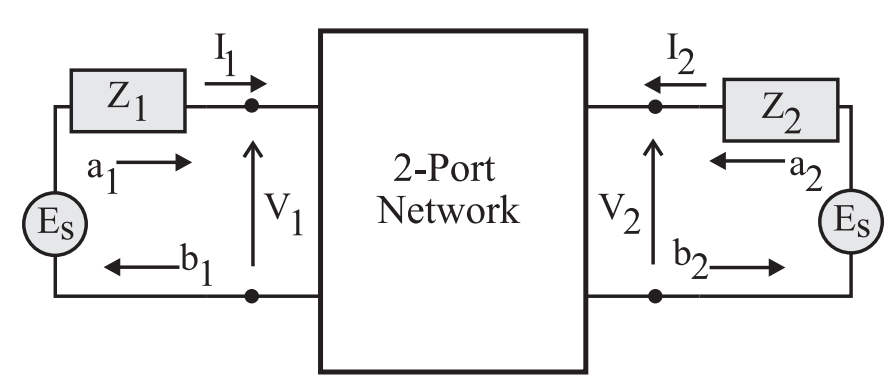

Figure 193: Illustration of 2-port network.

The s-parameters of a 2-port network are dependent on the reference impedances $\mathrm{Z}_{1}$ and $\mathrm{Z}_{2}$, therefore s-parameters are not unique for a given network [35]. It is 
seen from the above relations that when port $\mathrm{j}$ of the network is matched, $\mathrm{s}_{i i}$ and $\mathrm{S}_{j i}$ represent the reflection and transmission coefficients respectively. Therefore, it is desirable to have the reference impedances of the 2-port network match the impedance of the transmission lines and derive physical meaning from the s-parameters.

When simulating a coplanar waveguide (CPW) in Momentum with an internal port, the simulator uses a reference impedance of $50 \Omega$. Thus, if the characteristic impedance of the CPW line is different from $50 \Omega$, then $\mathrm{s}_{i i}$ can no longer be treated as the reflection coefficient looking into port $\mathrm{i}$ and $\mathrm{s}_{j i}$ cannot be treated as the transmission coefficient from port i to $\mathrm{j}$. The equations relating the impedance matrix and scattering matrix are given by

$$
\begin{gathered}
{[Z]=\left[\begin{array}{cc}
\sqrt{R_{\text {oldi }}} & 0 \\
0 & \sqrt{R_{\text {old } j}}
\end{array}\right]([I]-[S])^{-1}([S]+[I])\left[\begin{array}{cc}
\sqrt{R_{\text {oldi }}} & 0 \\
0 & \sqrt{R_{\text {old } j}}
\end{array}\right] \text { (50) }} \\
{[S]=\left(\left[\begin{array}{cc}
\frac{1}{\sqrt{R_{i}}} & 0 \\
0 & \frac{1}{\sqrt{R_{j}}}
\end{array}\right][Z]\left[\begin{array}{cc}
\frac{1}{\sqrt{R_{i}}} & 0 \\
0 & \frac{1}{\sqrt{R_{j}}}
\end{array}\right]-[I]\right)\left(\left[\begin{array}{cc}
\frac{1}{\sqrt{R_{i}}} & 0 \\
0 & \frac{1}{\sqrt{R_{j}}}
\end{array}\right][Z]\left[\begin{array}{cc}
\frac{1}{\sqrt{R_{i}}} & 0 \\
0 & \frac{1}{\sqrt{R_{j}}}
\end{array}\right]+[I]\right)^{-1}}
\end{gathered}
$$

where $[\mathrm{Z}]$ is the impedance matrix, $[\mathrm{S}]$ is the scattering matrix, and $[\mathrm{I}]$ is the identity matrix. Scattering parameters based on a given set of reference impedances can be converted to scattering parameters based on a new set of reference impedances. The conversion is achieved by converting the s-matrix to an impedance matrix (impedance matrix is independent of reference impedances and is therefore unique for a network) using Equation 50 with the old reference impedances $\mathrm{R}_{\text {oldi }}$ and $\mathrm{R}_{\text {oldj }}$ for ports $\mathrm{i}$ and j, respectively. A new s-matrix can then be derived using Equation 51, with the appropriate reference impedances $\mathrm{R}_{i}$ and $\mathrm{R}_{j}$ for ports $\mathrm{i}$ and $\mathrm{j}$, respectively[36]. 


\section{REFERENCES}

[1] Alcatel, "Alcatel 1916 datasheets."

[2] _ , "Alcatel 1915 datasheets."

[3] Jerry Sevick, Building and using baluns and ununs. Hicksville, NY: CQ Communications, Inc., 1994.

[4] J. W. Duncan and V. P. Minerva, "100:1 bandwidth balun transformer," Proc. of IRE, pp. 156-164, February 1960.

[5] John D. Kraus, Ronald J. Marhefka, Antennas for all applications, 3rd ed. New York, NY: McGraw-Hill, 2002.

[6] R. G. Titterington, "The ferrite-cored balun transformer," Radio Communication, pp. 216-220, March 1982.

[7] J. D. Gavenda, "Measured effectiveness of a toroid choke in reducing commonmode current," IEEE Symposium on EMC, pp. 208-210, May 1989.

[8] H. W. Ott, Noise reduction techniques in electronic systems, 2nd ed. New York, NY: John Wiley Sons, Inc., 1988.

[9] C. L. Ruthroff, "Some broadband transformers," Proceedings of the IRE, pp. 1337-1342, August 1959.

[10] R. B. Cowdell, "Don't experiment with ferrite beads...design with them." Electronic Design, pp. 100-103, June 1969.

[11] J. S. Belrose, "Transforming the balun," QST, pp. 30-34, June 1991.

[12] R. Sturdivant, "Balun designs for wireless, ...mixers, amplifiers and antennas," Applied Microwave, vol. 5, no. 3, pp. 34-42, Summer 1993.

[13] O. M. Woodward, Jr., "Balance measurements on balun transformers," Electronics, vol. 26, no. 9, pp. 188-191, September 1953.

[14] W. Bakalski, W. Simburger, H. Knapp, H.-D. Wohlmuth, and A. L. Scholtz, "Lumped and distributed lattice-type lc-baluns," IEEE MTT-S International, vol. 1, pp. 209-212, June 2002.

[15] V. Trifunovic and B. Jokanovic, "Double-y baluns for mmics and wireless applications," Microwave Journal, vol. 41, no. 1, pp. 70-92, January 1998. 
[16] B. Schiek and J. Kohler, "An improved microstrip-to-microslot transition," IEEE Trans. on MTT, vol. 24, no. 4, pp. 231-233, April 1976.

[17] V. Trifunovic and B. Jokanovic, "Four decade bandwidth uniplanar balun," Electronics Letters, vol. 28, no. 6, pp. 534-535, March 1992.

[18] — , "Review of printed marchand and double y baluns: characteristics and application," IEEE Trans. MTT, vol. 42, no. 8, pp. 1454-1462, August 1994.

[19] — , "New uniplanar balun," Electronics Letters, vol. 27, no. 10, pp. 813-815, May 1991.

[20] V. Trifunovic, A. Marincic, B. Jokanovic, "Analysis of the parasitic effects in double-y baluns," IEE Proc.-Microw. Antennas Propagation, vol. 148, no. 4, pp. 239-245, August 2001.

[21] B. Jokanovic, V. Trifunovic, B. Reljic, "Balance measurements in double-y baluns," IEE Proc.-Microw. Antennas Propagation, vol. 149, no. 56, pp. 257260, Oct-Nov 2002.

[22] H. Gu and K. Wu, "Broadband design consideration of uniplanar double-y baluns for hybrid and monolithic integrated circuits," IEEE MTT-S, pp. 863-866, 1999.

[23] K. C. Gupta, R. Garg, I. Bahl, P. Bhartia, Microstrip lines and slotlines, 2nd ed. Norwood, MA: John Wiley Sons, Inc., 1996.

[24] G. Ghione and C. Naldi, "Analytical formulas for coplanar lines in hybrid and monolithic mics," Electronics Letters, vol. 20, no. 4, pp. 179-181, February 1984.

[25] G. Ghione, "A cad-oriented analytical model for the losses of general asymmetric coplanar lines in hybrid and monolithic mics," IEEE Trans. MTT, vol. 41, no. 9, pp. 1499-1510, September 1993.

[26] A. K. Rastogi and A. K. Tiwari, "Attenuation characteristics of coplanar waveguides," Proc. Millimeter Wave and Far Infrared Science and Technology, pp. 157-160, August 1996.

[27] John L. Volakis, Arindam Chatterjee, and Leo C. Kempel, Finite Element Method for Electromagnetics. New York, NY: IEEE Press, 1998.

[28] I. Bardi and Z. J. Cendes, "New directions in hfss for designing microwave devices," Microwave Journal, vol. 41, no. 8, pp. 22-36, August 1998.

[29] I. Bardi, "Parameter estimation for pmls used with 3d finite element codes," IEEE Transactions on Magnetics, vol. 34, no. 5, pp. 2755-2758, September 1998.

[30] K. Kim and W. R. Scott, Jr., "A resistive linear antenna for ground-penetrating radars," SPIE Conference, 2004. 
[31] D. M. Pozar, Microwave Engineering, 2nd ed. New York: John Wiley Sons, Inc., 1998.

[32] T. T. Wu and R. W. P. King, "The cylindrical antenna with nonreflecting resistive loading," IEEE Transactions on AP, vol. 13, pp. 369-373, May 1965.

[33] C. A. Balanis, Antenna Theory Analysis and Design, 2nd ed. New York: John Wiley Sons, Inc., 1997.

[34] Minicircuits, "Minicircuits era-1sm datasheet," Tech. Rep.

[35] R. Mavaddat, Network Scattering Parameters. New Jersey: World Scientific, 1996, vol. 2 .

[36] R. J. Weber, Introduction to Microwave Circuits: Radio Frequency and Design Applications. New York: IEEE Press, 2001.

[37] J. S. McLean, "Balancing networks for symmetric antennas. i. classification and fundamental operation," IEEE Trans. EMC, vol. 44, pp. 503-514, November 2002. 


\section{VITA}

Jaikrishna Benny Venkatesan was born on February 2, 1976 in Madras, India. He received his high school education from Southwestern Educational Society, in Puerto Rico. He received his BSEE from Georgia Institute of Technology in 1998. During his undergraduate years, he was a Coop with Intel Corp. in California. From 1998 to 1999 he was employed as an RF Engineer at Motorola Space and Systems Technology Group in Arizona. He received his MSECE in 2001 from Georgia Institute of Technology. His research interests include microwave baluns, RF design, and radar applications. 